\title{
Asymmetric Transfer Hydrogenation of rac- $\alpha$-(Purin-9-yl)cyclopentones via Dynamic Kinetic Resolution for the Construction of Carbocyclic Nucleosides
}

Yi-Ming Zhang, Qi-Ying Zhang*, Dong-Chao Wang, Ming-Sheng Xie, Gui-Rong Qu, and Hai-Ming Guo*

Henan Key Laboratory of Organic Functional Molecules and Drug Innovation, Collaborative Innovation Center of Henan Province for Green Manufacturing of Fine Chemicals, School of Chemistry and Chemical Engineering, Henan Normal University, Xinxiang, Henan 453007, China.

E-mail: zhangqiying@htu.edu.cn; ghm@htu.edu.cn

\section{Table of Contents}

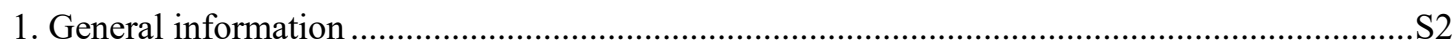

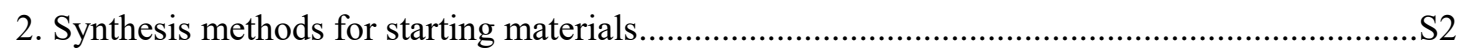

3. General procedure for asymmetric transfer hydrogenation of $\alpha$-(purin-9-yl)cycloalkanones.....S4

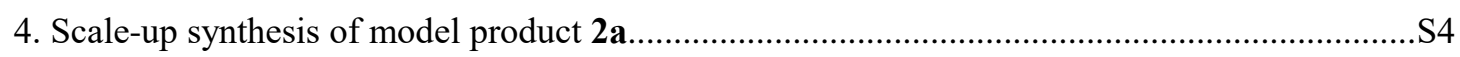

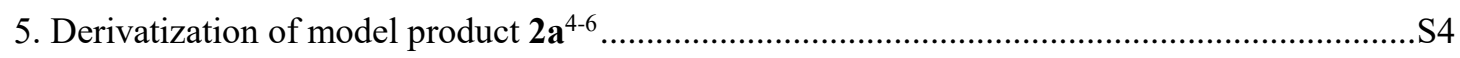

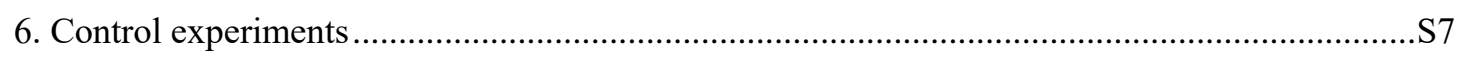

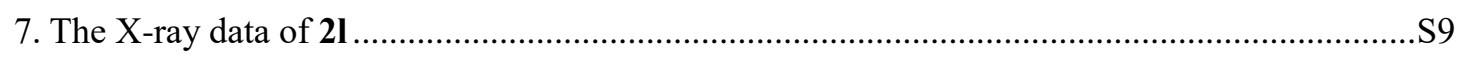

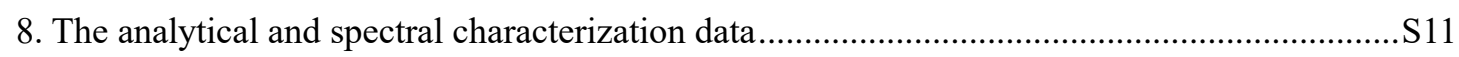

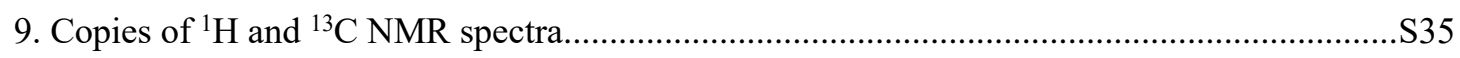

10. Copies of HPLC spectra for racemic and chiral products ....................................................S106

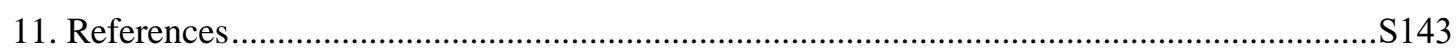




\section{General information}

${ }^{1} \mathrm{H}$ NMR and ${ }^{13} \mathrm{C}$ NMR spectra were recorded on Bruker Avance III HD 600 or Avance $400 \mathrm{MHz}$ spectrometer. Chemical shifts are recorded in ppm relative to tetramethylsilane with the solvent resonance as the internal standard. Data are represented as follows: chemical shift, multiplicity (s = singlet, $\mathrm{d}=$ doublet, $\mathrm{t}=$ triplet, $\mathrm{q}=$ quartet, $\mathrm{dd}=$ doublet of doublets, $\mathrm{m}=$ multiplet ), coupling constants $(J)$ are in Hertz $(\mathrm{Hz})$, and integration. Enantiomer excesses were determined by chiral HPLC analysis on Chiralcel IA/AS-H/ID/OD-H/IE in comparison with the authentic racemates. Chiral HPLC analysis was recorded on Thermo Scientific Dionex Ultimate 3000 and Agilent Technologies 1260 Infinity. Optical rotations were recorded on Autopol Automatic Polarimeter, and were reported as follows: $[\alpha]_{\mathrm{D}}{ }^{\mathrm{T}}$ (c: $\mathrm{g} / 100 \mathrm{~mL}$, in $\mathrm{CH}_{2} \mathrm{Cl}_{2}$ ). High resolution mass spectra (HRMS) was recorded on an ABI/Sciex QStar Mass Spectrometer (ESI). Single crystal X-ray crystallography data were obtained on Supernova Atlas S2 CCD detector. Melting point (m.p.) data were obtained on X-5 micro melting point apparatus. For column chromatography, silica gel (200-300 mesh) was used as the stationary phase.

\section{Synthesis methods for starting materials}

\section{(1) The synthesis of rac- $\alpha$-(purin-9-yl)cycloalkanones 1a-t ${ }^{1}$}

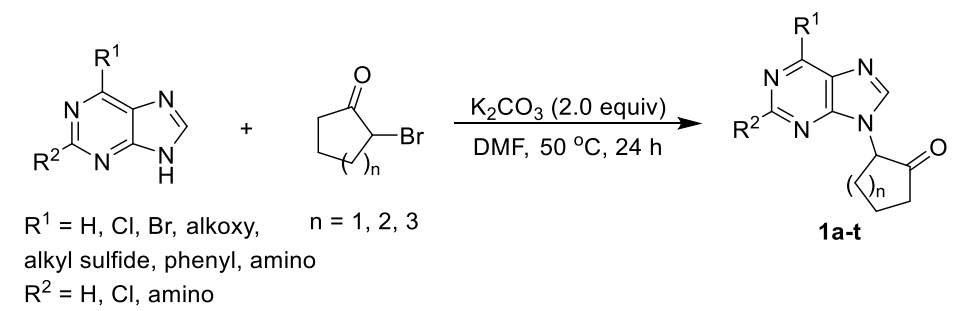

To a mixture of purine $(5.0 \mathrm{mmol})$ and $\mathrm{K}_{2} \mathrm{CO}_{3}(1.38 \mathrm{~g}, 10.0 \mathrm{mmol})$ in DMF $(10.0 \mathrm{~mL})$, the solution of $\alpha$-bromocycloalkanones $(10.0 \mathrm{mmol})$ in DMF $(10.0 \mathrm{~mL})$ was added. Then, the resulting mixture was stirred at $50{ }^{\circ} \mathrm{C}$ until the starting materials were consumed as indicated by TLC analysis. After being cooled down to room temperature, water $(100.0 \mathrm{~mL})$ and ethyl acetate $(50.0 \mathrm{~mL})$ was added into the mixture respectively. Subsequently, the separated aqueous layer was extracted with ethyl acetate $(50.0 \mathrm{~mL} \times 3)$. The combined organic layers were washed with brine $(100.0 \mathrm{~mL} \times 3)$, dried over anhydrous $\mathrm{Na}_{2} \mathrm{SO}_{4}$, filtered, and evaporated under reduced pressure. The residue was purified by flash column chromatography with petroleum ether: ethyl acetate (3:1) as the eluant to afford $r a c-\alpha$-(purin-9-yl)cycloalkanones 1a-t (30-85\% yields). 


\section{(2) The synthesis of rac- $\alpha$-N-heteroaromatic-substituted cyclopentones 3a-g}

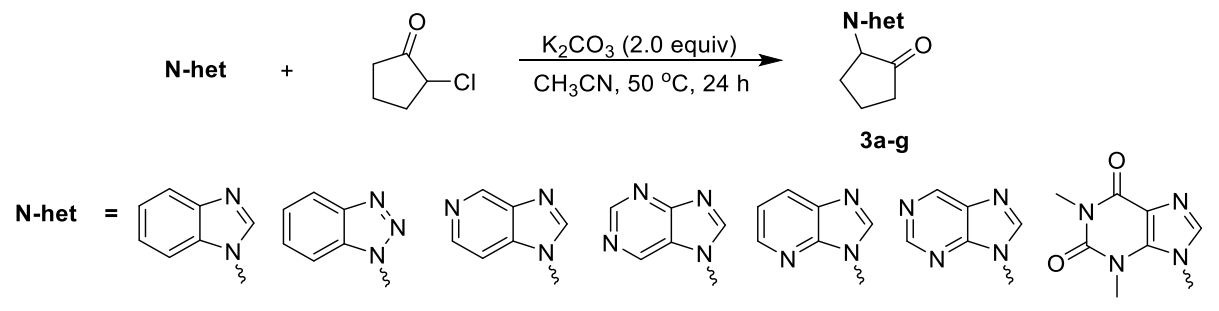

To a mixture of $\mathrm{N}$-heteroaromatics $(5.0 \mathrm{mmol})$ and $\mathrm{K}_{2} \mathrm{CO}_{3}(1.38 \mathrm{~g}, 10.0 \mathrm{mmol})$ in $\mathrm{CH}_{3} \mathrm{CN}$ $(10.0 \mathrm{~mL})$, the solution of $\alpha$-chlorocycloalkanones $(1.18 \mathrm{~g}, 10.0 \mathrm{mmol})$ in $\mathrm{CH}_{3} \mathrm{CN}(10.0 \mathrm{~mL})$ was added. Then the mixture was stirred at $50{ }^{\circ} \mathrm{C}$ until the starting materials were consumed as indicated by TLC analysis. After being cooled down to room temperature, the reaction mixture was filtered, concentrated under reduced pressure. The residue was purified by flash column chromatography with petroleum ether: ethyl acetate (2:1) as the eluant to afford products $\mathbf{3 a - g}$ (11-57\% yields).

(3) The synthesis of 2-allyl-2-(6-methoxy-9H-purin-9-yl)cyclopentan-1-one $3 \mathrm{~h}^{2}$ and

\section{2-(6-methoxy-9H-purin-9-yl)cyclopent-1-en-1-yl acetate $3 \mathbf{i}^{3}$}

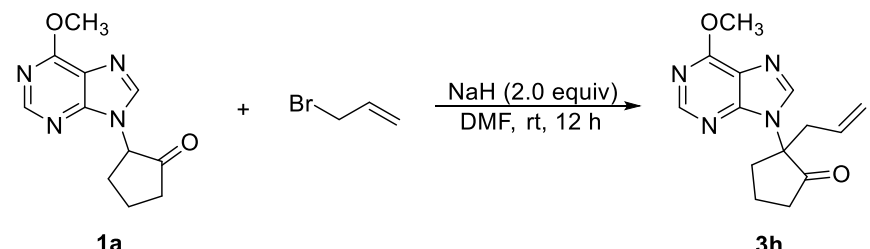

To a stirred solution of $1 \mathrm{a}(232.1 \mathrm{mg}, 1.0 \mathrm{mmol})$ in DMF $(5.0 \mathrm{~mL}), \mathrm{NaH}(60 \%$ dispersion in mineral oil, $48.0 \mathrm{mg}, 2.0 \mathrm{mmol})$ and allyl bromide $(239.9 \mathrm{mg}, 2.0 \mathrm{mmol})$ was added respectively. And the reaction was stirred at room temperature for $12 \mathrm{~h}$. Then water $(5.0 \mathrm{~mL})$ was added. Subsequently the resulting mixture was extracted with ethyl acetate $(5.0 \mathrm{~mL} \times 3)$, and the combined organic layers were washed with brine $(5.0 \mathrm{~mL} \times 3)$, dried over anhydrous $\mathrm{Na}_{2} \mathrm{SO}_{4}$, filtered, and evaporated under reduced pressure. The residue was purified by flash column chromatography with petroleum ether: ethyl acetate (2:1) as the eluant to afford the product $\mathbf{3 h}$ as white oil with $31 \%$ yield.

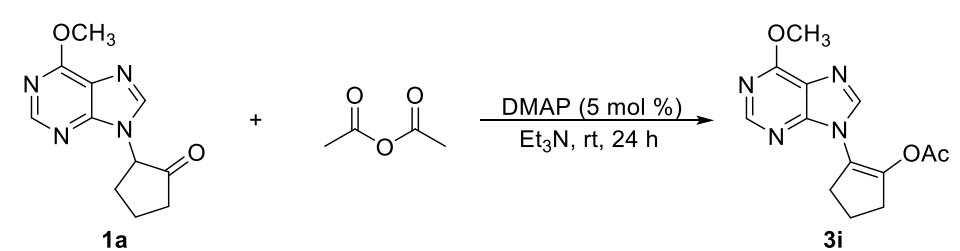

To a stirred solution of $1 \mathrm{a}(232.1 \mathrm{mg}, 1.0 \mathrm{mmol})$ in acetic anhydride $(5.0 \mathrm{~mL}), \mathrm{Et}_{3} \mathrm{~N}(303.6$ 
$\mathrm{mg}, 3.0 \mathrm{mmol})$ and DMAP $(6.2 \mathrm{mg}, 0.05 \mathrm{mmol})$ was added respectively. Then the reaction was stirred at room temperature for $24 \mathrm{~h}$. Subsequently the reaction mixture was filtered, concentrated under reduced pressure. The residue was purified by flash column chromatography with petroleum ether: ethyl acetate (2:1) as the eluant to afford the product $\mathbf{3 i}$ as a white solid with $53 \%$ yield.

\section{General procedure for asymmetric transfer hydrogenation of} $\alpha$-(purin-9-yl)cycloalkanones
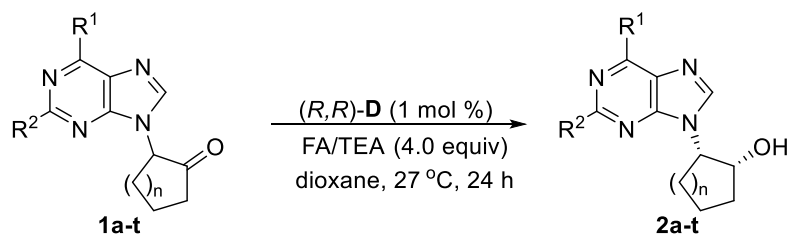

The $\alpha$-(purin-9-yl)cycloalkanones 1a-t $(0.1 \mathrm{mmol}),(R, R)-\mathbf{D}(0.7 \mathrm{mg}, 0.001 \mathrm{mmol})$, dioxane $(1.0 \mathrm{~mL})$ and FA/TEA (70 $\mu \mathrm{L}, 4.0$ equiv) was added into the reaction tube respectively. The mixture was stirred at $27{ }^{\circ} \mathrm{C}$ for $24 \mathrm{~h}$. Subsequently, the reaction mixture was filtered, concentrated under reduced pressure. The residue was purified by flash column chromatography with petroleum ether: ethyl acetate (2:1) as the eluant to afford the corresponding product 2a-t.

\section{Scale-up synthesis of model product 2 a}

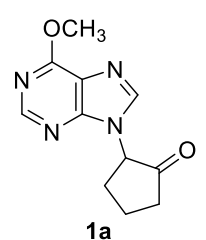

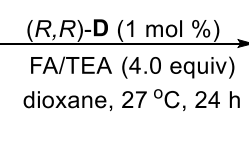

FA/TEA (4.0 equiv)

(a)

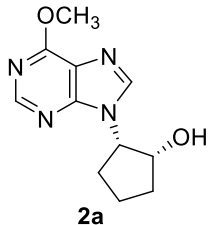

2a

The $\alpha$-purine cyclopentone $1 \mathrm{a}(1.16 \mathrm{~g}, 5.0 \mathrm{mmol}),(R, R)-\mathbf{D}(35.6 \mathrm{mg}, 0.005 \mathrm{mmol})$, dioxane $(30.0 \mathrm{~mL})$ and FA/TEA $(3.5 \mathrm{~mL}$, ) was added to the reaction tube respectively. Then the reaction was stirred at $27{ }^{\circ} \mathrm{C}$ for $24 \mathrm{~h}$. Subsequently, the reaction mixture was filtered, concentrated under reduced pressure. The residue was purified by flash column chromatography with petroleum ether: ethyl acetate (2:1) as the eluant to afford the product $\mathbf{2 a}$ as white solid $(1.01 \mathrm{~g}, 86 \%$ yield, $>99: 1$ dr, $97 \%$ ee).

\section{Derivatization of model product $2 a^{4-6}$}



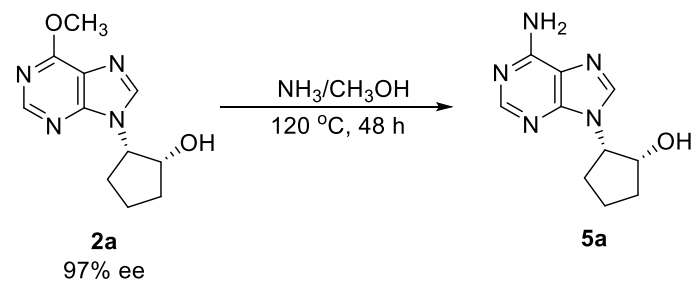

To a solution of $2 \mathrm{a}(23.4 \mathrm{mg}, 0.1 \mathrm{mmol}), \mathrm{NH}_{3} / \mathrm{MeOH}(2.0 \mathrm{~mL})$ was added. Then the reaction was stirred at $120^{\circ} \mathrm{C}$ for $48 \mathrm{~h}$. After being cooled down to room temperature, the mixture was concentrated under reduced pressure to give a residue. The obtained residue was purified by flash chromatography on silica gel with DCM:MeOH (30:1) as the eluant to afford

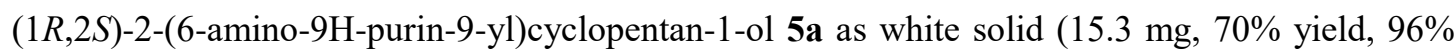
ee).

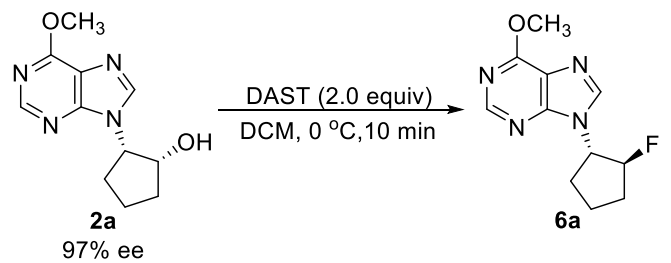

To a solution of 2a $(23.4 \mathrm{mg}, 0.1 \mathrm{mmol})$ in DCM $(1.0 \mathrm{~mL})$ at $0{ }^{\circ} \mathrm{C}$, DAST $(26.7 \mu \mathrm{L}, 0.2$ mmol) was added. Then the reaction was stirred at $0{ }^{\circ} \mathrm{C}$ for $10 \mathrm{~min}$. The mixture was concentrated under reduced pressure. The obtained residue was directly purified by flash chromatography on silica gel with petroleum ether: ethyl acetate (2:1) to afford the product $\mathbf{6 a}$ as white solid (16.8 $\mathrm{mg}$, $71 \%$ yield, $98 \%$ ee).

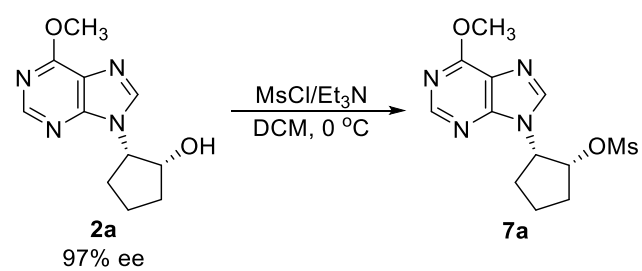

To a solution of the compound $2 \mathrm{a}(117.0 \mathrm{mg}, 0.5 \mathrm{mmol})$ and $\mathrm{Et}_{3} \mathrm{~N}(173.0 \mu \mathrm{L}, 1.25 \mathrm{mmol})$ in $\operatorname{DCM}(10.0 \mathrm{~mL})$ at $0{ }^{\circ} \mathrm{C}$, mesyl chloride $(58.0 \mu \mathrm{L}, 0.75 \mathrm{mmol})$ was added dropwise under stirring. After the reaction was stirred for $4 \mathrm{~h}$, the reaction solution was concentrated under reduced pressure to give residue. The obtained residue was purified by flash chromatography on a silica gel column using eluent ( $\mathrm{DCM}: \mathrm{MeOH}=80: 1)$ to give the product $7 \mathbf{a}$ as a white solid (151.4 $\mathrm{mg}$, 97\% yield, $98 \%$ ee). 


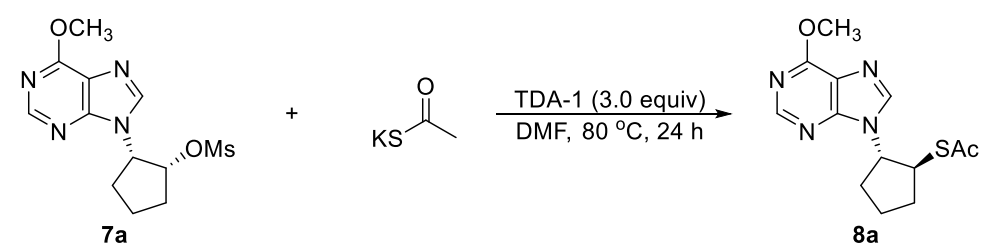

To a solution of compound 7a $(31.2 \mathrm{mg}, 0.1 \mathrm{mmol})$ and AcSK $(22.8 \mathrm{mg}, 0.2 \mathrm{mmol})$ in DMF $(1.0 \mathrm{~mL})$, TDA-1 $(96.0 \mu \mathrm{L}, 0.3 \mathrm{mmol})$ was added under stirring. Then the reaction was stirred at $80^{\circ} \mathrm{C}$ for $24 \mathrm{~h}$. After the reaction was complete as monitored by TLC, water $(5.0 \mathrm{~mL})$ was added into the mixture. The resulting mixture was extracted with ethyl acetate $(5.0 \mathrm{~mL} \times 3)$. The combined organic layers were washed with brine $(5.0 \mathrm{~mL} \times 3)$, dried over anhydrous $\mathrm{Na}_{2} \mathrm{SO}_{4}$, filtered, and evaporated under reduced pressure. The obtained residue was purified by flash chromatography on a silica gel column using eluent (petroleum ether: ethyl acetate $=2: 1$ ) to give the product $8 \mathbf{a}$ as white oil ( $22.2 \mathrm{mg}, 76 \%$ yield, $98 \%$ ee).

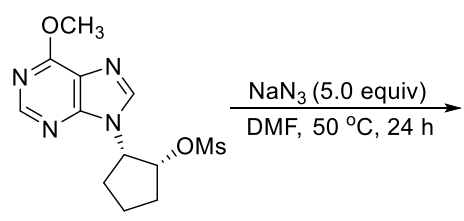

$7 a$

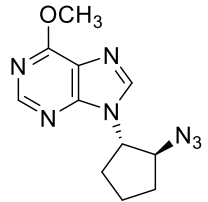

$9 a$

To a cold solution of $7 \mathbf{a}(93.6 \mathrm{mg}, 0.3 \mathrm{mmol})$ in DMF $(3.0 \mathrm{~mL})$ at $0{ }^{\circ} \mathrm{C}, \mathrm{NaN}_{3}(97.5 \mathrm{mg}, 1.5$ mmol) was added, and then the reaction was stirred at $50{ }^{\circ} \mathrm{C}$ for $24 \mathrm{~h}$. After being cooled down to room temperature, water $(10.0 \mathrm{~mL})$ was added into the mixture. The resulting mixture was extracted with ethyl acetate $(10.0 \mathrm{~mL} \times 3)$. The combined organic layers were washed with brine $(10.0 \mathrm{~mL} \times 3)$, dried over anhydrous $\mathrm{Na}_{2} \mathrm{SO}_{4}$, filtered, and evaporated under reduced pressure. The obtained residue was purified by flash chromatography on a silica gel column using eluent (petroleum ether: ethyl acetate $=2: 1)$ to give the product $9 \mathrm{a}$ as yellow oil $(67.6 \mathrm{mg}, 87 \%$ yield, $97 \%$ ee).

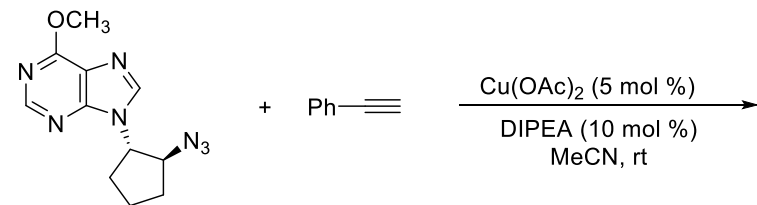

9a

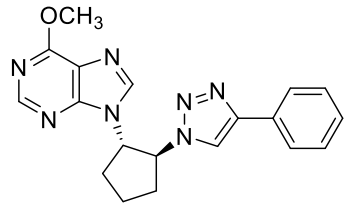

$10 a$

To a solution of the compound 9a $(25.9 \mathrm{mg}, 0.1 \mathrm{mmol})$ in acetonitrile $(1.0 \mathrm{~mL})$, phenylacetylene $(16.5 \mu \mathrm{L}, 0.15 \mathrm{mmol})$ and $\mathrm{Cu}(\mathrm{OAc})_{2}(0.9 \mathrm{mg}, 0.005 \mathrm{mmol})$ were added respectively. The mixture was stirred until $\mathrm{Cu}(\mathrm{OAc})_{2}$ was completely dissolved. Subsequently, the DIPEA $(1.7 \mu \mathrm{L}, 0.01 \mathrm{mmol})$ was added via syringe, and the solution allowed to react for $6 \mathrm{~h}$. 
Water $(2.0 \mathrm{~mL})$ was added into the mixture. The resulting mixture was extracted with ethyl acetate $(5.0 \mathrm{~mL} \times 3)$. The combined organic layers were washed with brine $(5.0 \mathrm{~mL} \times 3)$, dried over anhydrous $\mathrm{Na}_{2} \mathrm{SO}_{4}$, filtered, and evaporated under reduced pressure. The obtained residue was purified by flash chromatography on a silica gel column using eluent (petroleum ether: ethyl acetate $=2: 1)$ to give the product $10 \mathrm{a}$ as a white oil $(33.2 \mathrm{mg}, 92 \%$ yield, $98 \%$ ee) .

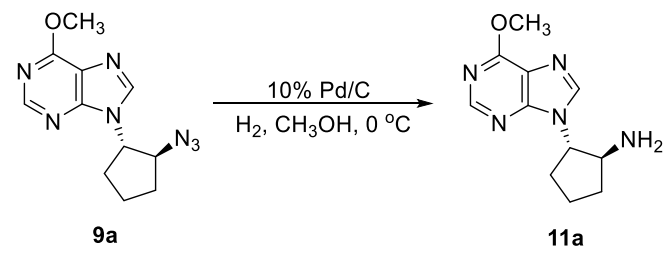

To a solution of the compound $9 \mathrm{a}(25.9 \mathrm{mg}, 0.1 \mathrm{mmol})$ in $\mathrm{MeOH}(1.0 \mathrm{~mL})$, the $10 \mathrm{wt} \% \mathrm{Pd} / \mathrm{C}$ (5.0 mg) was added. The reaction was stirred under $\mathrm{H}_{2}$ atmosphere $(1.0 \mathrm{~atm})$ at $0{ }^{\circ} \mathrm{C}$ for $12 \mathrm{~h}$. The mixture was filtrated through a pad of celite, rinsed with ethyl acetate $(10.0 \mathrm{~mL})$ and concentrated under reduced pressure. The obtained residue was purified by flash column chromatography (DCM:MeOH $=60: 1)$ to give the desired product 11a as yellow oil (20.0 mg, 86\% yield, 97\% ee).

\section{Control experiments}

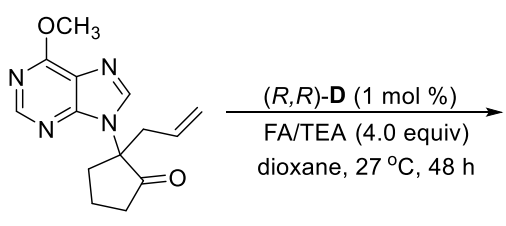

rac-3h

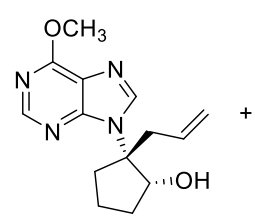

$4 h$

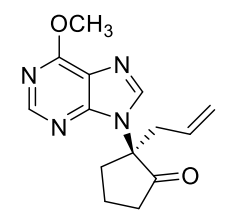

s-3h

The compound $\mathbf{r a c}-3 \mathrm{~h}(272.1 \mathrm{mg}, 0.1 \mathrm{mmol}),(R, R)-\mathbf{D}(0.7 \mathrm{mg}, 0.001 \mathrm{mmol})$, dioxane $(1.0$ $\mathrm{mL})$ and FA/TEA $(70.0 \mu \mathrm{L}, 0.4 \mathrm{mmol})$ was added into the reaction tube respectively. The reaction was stirred at $27{ }^{\circ} \mathrm{C}$ for $48 \mathrm{~h}$. Subsequently, the reaction mixture was filtered, concentrated under reduced pressure. The obtained residue was purified by flash column chromatography with petroleum ether: ethyl acetate $(2: 1)$ as the eluant to afford the product $4 \mathbf{h}$ as white oil (43\% yield and $99 \%$ ee) and $S-3 \mathbf{h}$ as white oil ( $45 \%$ yield, $99 \%$ ee).

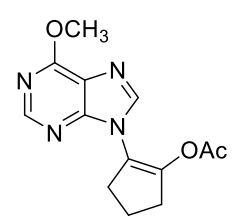

$3 i$

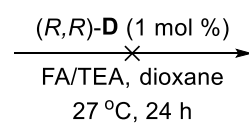

$27^{\circ} \mathrm{C}, 24 \mathrm{~h}$

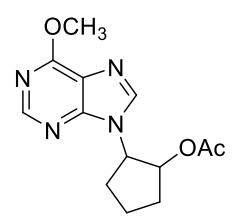

$4 i$

The 2-(6-methoxy-9H-purin-9-yl)cyclopent-1-en-1-yl acetate $3 \mathbf{i}(27.4 \mathrm{mg}, 0.1 \mathrm{mmol})$, $(R, R)-\mathbf{D}(0.7 \mathrm{mg}, 0.001 \mathrm{mmol})$, dioxane $(1.0 \mathrm{~mL})$ and FA/TEA $(70.0 \mu \mathrm{L}, 0.4 \mathrm{mmol})$ was added 
into the reaction tube respectively. The reaction was stirred at $27^{\circ} \mathrm{C}$ for $24 \mathrm{~h}$. The reaction was monitored by TLC, and no desired product was observed. 


\section{The X-ray data of 21}

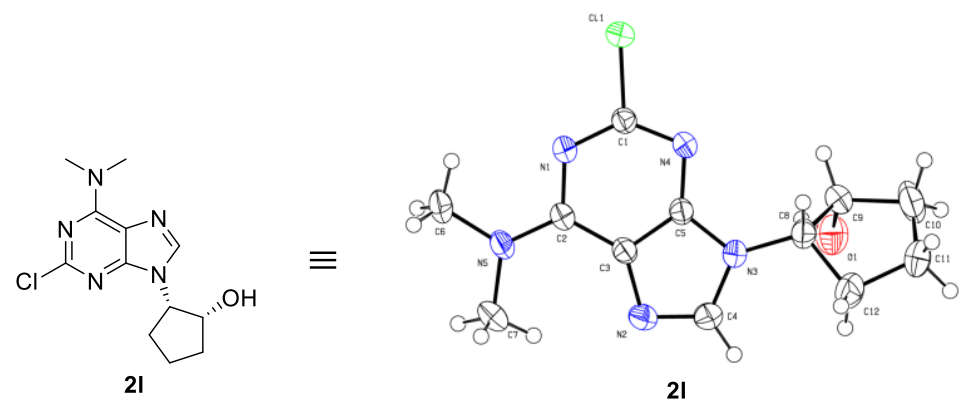

The chiral product of $\mathbf{2 l}$ was recrystallized by petroleum ether/diethyl ether/dichloromethane (2/1/1). CCDC 1866376 (2I) contains the supplementary crystallographic data for this paper. These data can be obtained free of charge from The Cambridge Crystallographic Data Centre via www.ccdc.cam.ac.uk/data_request/cif.

\section{Table 1 Crystal data and structure refinement for 21 .}

Identification code

Empirical formula

Formula weight

Temperature/K

Crystal system

Space group

$\mathrm{a} / \AA ̊ \AA$

$\mathrm{b} / \mathrm{A}$

$\mathrm{c} / \AA ̊ \AA$

$\alpha /^{\circ}$

$\beta /{ }^{\circ}$

$\gamma /{ }^{\circ}$

Volume $/ \AA^{3}$

Z

$\rho_{\text {calc }} \mathrm{g} / \mathrm{cm}^{3}$

$\mu / \mathrm{mm}^{-1}$

$\mathrm{F}(000)$

Crystal size $/ \mathrm{mm}^{3}$

Radiation

$2 \Theta$ range for data collection $/{ }^{\circ}$

Index ranges

Reflections collected

Independent reflections

Data/restraints/parameters
2I

$\mathrm{C}_{12} \mathrm{H}_{16} \mathrm{ClN}_{5} \mathrm{O}$

281.75

293(2)

monoclinic

$\mathrm{P} 2{ }_{1}$

$8.73422(6)$

6.98291(7)

10.56023(9)

90

92.8801(7)

90

$643.258(10)$

2

1.455

2.642

296.0

$0.23 \times 0.21 \times 0.2$

$\mathrm{CuK} \alpha(\lambda=1.54178)$

8.384 to 145.236

$-10 \leq \mathrm{h} \leq 10,-8 \leq \mathrm{k} \leq 8,-13 \leq 1 \leq 13$

19087

$2484\left[R_{\text {int }}=0.0414, R_{\text {sigma }}=0.0184\right]$

$2484 / 1 / 175$ 
Goodness-of-fit on $\mathrm{F}^{2}$

Final $\mathrm{R}$ indexes $[\mathrm{I}>=2 \sigma(\mathrm{I})]$

Final $\mathrm{R}$ indexes [all data]

Largest diff. peak/hole / e $\AA^{-3}$

Flack parameter
0.966

$\mathrm{R}_{1}=0.0340, \mathrm{wR}_{2}=0.0957$

$\mathrm{R}_{1}=0.0342, \mathrm{wR}_{2}=0.0959$

$0.20 /-0.27$

$-0.005(8)$ 


\section{The analytical and spectral characterization data}

\section{(1) The analytical and spectral characterization data of starting materials}

rac-2-(6-Methoxy-9H-purin-9-yl)cyclopentan-1-one (1a)

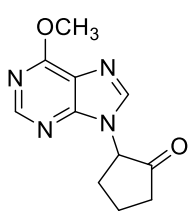

Yellow solid, m.p. $123.1-127.5{ }^{\circ} \mathrm{C} ; 0.55 \mathrm{~g}, 47 \%$ yield; ${ }^{1} \mathbf{H}$ NMR $\left(600 \mathrm{MHz}, \mathrm{CDCl}_{3}\right) \delta 8.46(\mathrm{~s}, 1 \mathrm{H})$, $7.86(\mathrm{~s}, 1 \mathrm{H}), 4.94(\mathrm{dd}, J=12.6,8.4 \mathrm{~Hz}, 1 \mathrm{H}), 4.16(\mathrm{~s}, 3 \mathrm{H}), 2.67-2.62(\mathrm{~m}, 1 \mathrm{H}), 2.57-2.54(\mathrm{~m}, 2 \mathrm{H})$, 2.49-2.42 (m, 1H), 2.32-2.27 (m, 1H), 2.06-1.98 (m, 1H). ${ }^{13} \mathbf{C}$ NMR (100 MHz, CDCl $) \delta$ 211.0, 161.1, 151.9, 151.8, 141.5, 121.5, 61.1, 54.3, 35.8, 29.5, 18.5; HRMS (ESI-TOF): exact mass calcd for $\mathrm{C}_{11} \mathrm{H}_{13} \mathrm{~N}_{4} \mathrm{O}_{2}(\mathrm{M}+\mathrm{H})^{+}$requires m/z 233.1033, found $\mathrm{m} / \mathrm{z} 233.1033$.

rac-2-(6-Ethoxy-9H-purin-9-yl)cyclopentan-1-one (1b)

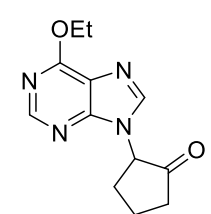

White oil, 0.63 g, 51\% yield; ${ }^{1} \mathbf{H}$ NMR (400 MHz, $\left.\mathrm{CDCl}_{3}\right) \delta 8.47$ (s, 1H), 7.86 (s, 1H), 4.96 (dd, $J$ $=12.4,8.4 \mathrm{~Hz}, 1 \mathrm{H}), 4.66(\mathrm{q}, J=7.2 \mathrm{~Hz}, 2 \mathrm{H}), 2.72-2.65(\mathrm{~m}, 1 \mathrm{H}), 2.61-2.57(\mathrm{~m}, 2 \mathrm{H}), 2.50-2.44(\mathrm{~m}$, 1H), 2.38-2.30 (m, 1H), 2.10-2.01 (m, 1H), $1.51(\mathrm{t}, J=7.2 \mathrm{~Hz}, 3 \mathrm{H}) .{ }^{13} \mathbf{C ~ N M R}\left(100 \mathrm{MHz}, \mathrm{CDCl}_{3}\right)$ $\delta$ 210.9, 161.1, 152.1, 152.0, 141.3, 121.7, 63.3, 61.2, 35.9, 29.7, 18.7, 14.6; HRMS (ESI-TOF): exact mass calcd for $\mathrm{C}_{12} \mathrm{H}_{15} \mathrm{~N}_{4} \mathrm{O}_{2}(\mathrm{M}+\mathrm{H})^{+}$requires $\mathrm{m} / \mathrm{z} 247.1190$, found $\mathrm{m} / \mathrm{z}$ 247.1191.

rac-2-(6-(Propylthio)-9H-purin-9-yl)cyclopentan-1-one (1c)

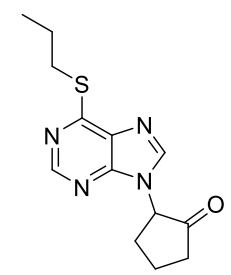

Yellow oil, 0.55 g, 40\% yield; ${ }^{1} \mathbf{H}$ NMR (600 MHz, $\left.\mathrm{CDCl}_{3}\right) \delta 8.61$ (s, 1H), 7.89 (s, 1H), 4.93 (dd, $J=12.6,8.4 \mathrm{~Hz}, 1 \mathrm{H}), 3.34(\mathrm{t}, J=7.8 \mathrm{~Hz}, 2 \mathrm{H}), 2.65-2.60(\mathrm{~m}, 1 \mathrm{H}), 2.56-2.53(\mathrm{~m}, 2 \mathrm{H}), 2.48-2.41(\mathrm{~m}$, 1H), 2.31-2.26 (m, 1H), 2.05-1.96 (m, 1H), 1.80-1.77 (m, 2H), $1.05(\mathrm{t}, J=7.8 \mathrm{~Hz}, 3 \mathrm{H}) .{ }^{13} \mathbf{C}$ NMR $\left(100 \mathrm{MHz}, \mathrm{CDCl}_{3}\right) \delta 210.9,161.8,151.7,148.2,141.8,131.4,61.1,35.8,30.7,29.6,22.9,18.6$, 13.5; HRMS (ESI-TOF): exact mass calcd for $\mathrm{C}_{13} \mathrm{H}_{17} \mathrm{~N}_{4} \mathrm{OS}(\mathrm{M}+\mathrm{H})^{+}$requires $\mathrm{m} / \mathrm{z} 277.1118$, found $\mathrm{m} / \mathrm{z} 277.1117$. 
rac-2-(6-Phenyl-9H-purin-9-yl)cyclopentan-1-one (1d)

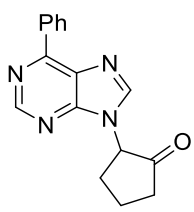

White oil, 0.42 g, 30\% yield; ${ }^{1} \mathbf{H}$ NMR (600 MHz, $\left.\mathrm{CDCl}_{3}\right) \delta 8.96$ (s, 1H), 8.76-8.75 (m, 2H), 8.07 (s, 1H), 7.57-7.52 (m, 3H), $5.03(\mathrm{dd}, J=12.6,8.4 \mathrm{~Hz}, 1 \mathrm{H}), 2.72-2.67(\mathrm{~m}, 1 \mathrm{H}), 2.62-2.59(\mathrm{~m}, 2 \mathrm{H})$, 2.54-2.48 (m, 1H), 2.36-2.31 (m, 1H), 2.10-2.02 (m, 1H). ${ }^{13} \mathbf{C}$ NMR (100 MHz, CDCl $) \delta 210.9$, $155.3,152.4,152.3,143.6,135.7,131.2,131.1,129.9,128.8,61.1,35.9,29.6,18.7$; HRMS (ESI-TOF): exact mass calcd for $\mathrm{C}_{16} \mathrm{H}_{15} \mathrm{~N}_{4} \mathrm{O}(\mathrm{M}+\mathrm{H})^{+}$requires m/z 279.1240, found m/z 279.1240. rac-2-(6-(Dimethylamino)-9H-purin-9-yl)cyclopentan-1-one (1e)

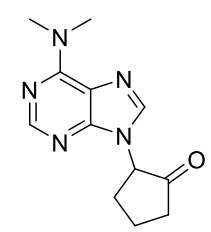

White solid, m.p. 130.3-136.0 ${ }^{\circ} \mathrm{C} ; 0.51 \mathrm{~g}, 42 \%$ yield; ${ }^{1} \mathbf{H}$ NMR $\left(600 \mathrm{MHz}, \mathrm{CDCl}_{3}\right) \delta 8.26(\mathrm{~s}, 1 \mathrm{H})$, $7.64(\mathrm{~s}, 1 \mathrm{H}), 4.90(\mathrm{dd}, J=12.6,8.4 \mathrm{~Hz}, 1 \mathrm{H}), 3.50(\mathrm{~s}, 6 \mathrm{H}), 2.63-2.58(\mathrm{~m}, 1 \mathrm{H}), 2.54-2.51(\mathrm{~m}, 2 \mathrm{H})$, 2.42-3.35 (m, 1H), 2.29-2.23 (m, 1H), 2.03-1.96 (m, 1H). ${ }^{13} \mathbf{C}$ NMR (100 MHz, $\left.\mathrm{CDCl}_{3}\right) \delta 211.5$, 155.1, 152.3, 150.6, 137.2, 120.2, 60.8, 38.7, 35.9, 29.8, 18.6; HRMS (ESI-TOF): exact mass calcd for $\mathrm{C}_{12} \mathrm{H}_{16} \mathrm{~N}_{5} \mathrm{O}(\mathrm{M}+\mathrm{H})^{+}$requires $\mathrm{m} / \mathrm{z} 246.1349$, found $\mathrm{m} / \mathrm{z} 246.1350$.

rac-2-(6-(Diethylamino)-9H-purin-9-yl)cyclopentan-1-one (1f)

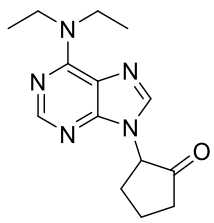

Yellow oil, 0.60 g, 44\% yield; ${ }^{1} \mathbf{H}$ NMR (600 MHz, $\left.\mathrm{CDCl}_{3}\right) \delta 8.27(\mathrm{~s}, 1 \mathrm{H}), 7.65$ (s, 1H), 4.92 (dd, $J=12.6,8.4 \mathrm{~Hz}, 1 \mathrm{H}), 3.97(\mathrm{~s}, 4 \mathrm{H}), 2.66-2.61(\mathrm{~m}, 1 \mathrm{H}), 2.58-2.55(\mathrm{~m}, 2 \mathrm{H}), 2.44-2.37(\mathrm{~m}, 1 \mathrm{H})$, 2.32-2.26 (m, 1H), 2.06-1.97 (m, 1H), $1.29(\mathrm{t}, J=7.2 \mathrm{~Hz}, 6 \mathrm{H}) .{ }^{13} \mathbf{C ~ N M R}\left(100 \mathrm{MHz}, \mathrm{CDCl}_{3}\right) \delta$ 211.6, 153.8, 152.4, 150.5, 137.3, 119.5, 60.7, 43.1, 35.8, 29.6, 18.5, 13.5; HRMS (ESI-TOF): exact mass calcd for $\mathrm{C}_{14} \mathrm{H}_{20} \mathrm{~N}_{5} \mathrm{O}(\mathrm{M}+\mathrm{H})^{+}$requires $\mathrm{m} / \mathrm{z} 274.1662$, found $\mathrm{m} / \mathrm{z} 274.1662$. rac-2-(6-(Pyrrolidin-1-yl)-9H-purin-9-yl)cyclopentan-1-one (1g) 


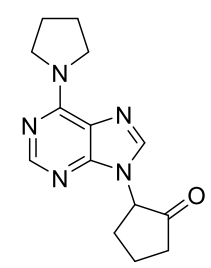

Yellow oil, 0.42 g, 31\% yield; ${ }^{1} \mathbf{H}$ NMR $\left(600 \mathrm{MHz}, \mathrm{CDCl}_{3}\right) \delta 8.29$ (s, 1H), $7.65(\mathrm{~s}, 1 \mathrm{H}), 4.92(\mathrm{dd}$, $J=12.6,9.0 \mathrm{~Hz}, 1 \mathrm{H}), 4.16(\mathrm{~s}, 2 \mathrm{H}), 3.75-3.72(\mathrm{~m}, 2 \mathrm{H}), 2.66-2.61(\mathrm{~m}, 1 \mathrm{H}), 2.57-2.54(\mathrm{~m}, 2 \mathrm{H})$, 2.45-2.38 (m, 1H), 2.31-2.58 (m, 1H), 2.05-1.97 (m, 5H). ${ }^{13} \mathbf{C}$ NMR (100 MHz, $\left.\mathrm{CDCl}_{3}\right) \delta$ 211.5, 153.3, 152.8, 150.2, 137.8, 120.3, 60.8, 35.9, 29.8, 26.3, 24.5, 18.6; HRMS (ESI-TOF): exact mass calcd for $\mathrm{C}_{14} \mathrm{H}_{18} \mathrm{~N}_{5} \mathrm{O}(\mathrm{M}+\mathrm{H})^{+}$requires m/z 272.1506, found m/z 272.1507.

rac-2-(6-(Piperidin-1-yl)-9H-purin-9-yl)cyclopentan-1-one (1h)

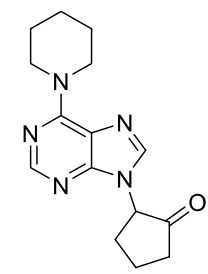

Yellow oil, $0.50 \mathrm{~g}, 39 \%$ yield; ${ }^{1} \mathbf{H}$ NMR $\left(600 \mathrm{MHz}, \mathrm{CDCl}_{3}\right) \delta 8.18-8.17(\mathrm{~m}, 1 \mathrm{H}), 7.60-7.59(\mathrm{~m}$, $1 \mathrm{H}), 4.86-4.82(\mathrm{~m}, 1 \mathrm{H}), 4.15(\mathrm{~s}, 4 \mathrm{H}), 2.53-2.49(\mathrm{~m}, 1 \mathrm{H}), 2.47-2.43(\mathrm{~m}, 2 \mathrm{H}), 2.36-2.28(\mathrm{~m}, 1 \mathrm{H})$, 2.20-2.14 (m, 1H), 1.94-1.87 (m, 1H), 1.65-1.58 (m, 6H). ${ }^{13} \mathbf{C}$ NMR (100 MHz, $\left.\mathrm{CDCl}_{3}\right) \delta 211.4$, $153.8,152.2,150.6,136.9,119.6,60.6,46.3,35.7,29.5,26.1,24.7,18.4$; HRMS (ESI-TOF): exact mass calcd for $\mathrm{C}_{15} \mathrm{H}_{20} \mathrm{~N}_{5} \mathrm{O}(\mathrm{M}+\mathrm{H})^{+}$requires $\mathrm{m} / \mathrm{z}$ 286.1662, found $\mathrm{m} / \mathrm{z}$ 286.1665.

rac-2-(6-Morpholino-9H-purin-9-yl)cyclopentan-1-one (1i)

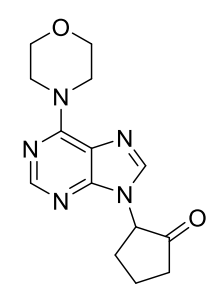

Yellow oil, 0.57 g, 40\% yield; ${ }^{1} \mathbf{H}$ NMR $\left(400 \mathrm{MHz}, \mathrm{CDCl}_{3}\right) \delta 8.26(\mathrm{~s}, 1 \mathrm{H}), 7.66(\mathrm{~s}, 1 \mathrm{H}), 4.90(\mathrm{dd}$, $J=12.4,8.4 \mathrm{~Hz}, 1 \mathrm{H}), 4.29-4.26(\mathrm{~m}, 4 \mathrm{H}), 3.79(\mathrm{t}, J=4.8 \mathrm{~Hz}, 4 \mathrm{H}), 2.64-2.51(\mathrm{~m}, 3 \mathrm{H}), 2.44-2.35(\mathrm{~m}$, 1H), 2.30-2.22 (m, 1H), 2.05-1.94 (m, 1H). ${ }^{13} \mathbf{C}$ NMR (100 MHz, $\left.\mathrm{CDCl}_{3}\right) \delta$ 211.3, 154.0, 152.3, 150.9, 137.6,119.9, 67.1, 60.8, 45.7, 35.9, 29.7, 18.6; HRMS (ESI-TOF): exact mass calcd for $\mathrm{C}_{14} \mathrm{H}_{18} \mathrm{~N}_{5} \mathrm{O}_{2}(\mathrm{M}+\mathrm{H})^{+}$requires $\mathrm{m} / \mathrm{z}$ 288.1455, found $\mathrm{m} / \mathrm{z} 288.1456$.

rac-2-(6-Bromo-9H-purin-9-yl)cyclopentan-1-one (1j) 


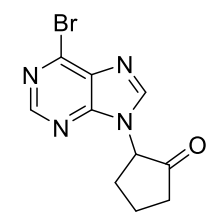

White solid, m.p. $141.5-146.3^{\circ} \mathrm{C} ; 0.99 \mathrm{~g}, 71 \%$ yield; ${ }^{1} \mathbf{H}$ NMR $\left(400 \mathrm{MHz}, \mathrm{CDCl}_{3}\right) \delta 8.64(\mathrm{~s}, 1 \mathrm{H})$, $8.10(\mathrm{~s}, 1 \mathrm{H}), 4.99(\mathrm{dd}, J=12.4,8.4 \mathrm{~Hz}, 1 \mathrm{H}), 2.74-2.67(\mathrm{~m}, 1 \mathrm{H}), 2.62-2.44(\mathrm{~m}, 3 \mathrm{H}), 2.39-2.32(\mathrm{~m}$, $1 \mathrm{H}), 2.14-2.01(\mathrm{~m}, 1 \mathrm{H}) .{ }^{13} \mathrm{C}$ NMR $\left(150 \mathrm{MHz}, \mathrm{CDCl}_{3}\right) \delta 210.2,151.9,150.5,144.4,143.5,134.4$, 61.6, 35.8, 29.5, 18.7; HRMS (ESI-TOF): exact mass calcd for $\mathrm{C}_{10} \mathrm{H}_{10} \mathrm{BrN}_{4} \mathrm{O}(\mathrm{M}+\mathrm{H})^{+}$requires $\mathrm{m} / \mathrm{z} 281.0032$, found $\mathrm{m} / \mathrm{z} 281.0034$.

rac-2-(6-Chloro-9H-purin-9-yl)cyclopentan-1-one (1k)<smiles>O=C1CCCC1n1cnc2c(Cl)ncnc21</smiles>

Yellow oil, 1.00 g, $85 \%$ yield; ${ }^{1} \mathbf{H}$ NMR $\left(600 \mathrm{MHz}, \mathrm{CDCl}_{3}\right) \delta 8.69(\mathrm{~s}, 1 \mathrm{H}), 8.09(\mathrm{~s}, 1 \mathrm{H}), 5.02-4.98$ (m, 1H), 2.73-2.69 (m, 1H), 2.65-2.56 (m, 2H), 2.54-2.48 (m, 1H), 2.38-2.35 (m, 1H), 2.10-2.08 (m, 1H). ${ }^{13} \mathbf{C}$ NMR $\left(150 \mathrm{MHz}, \mathrm{CDCl}_{3}\right) \delta 210.3,151.9,151.7,151.5,144.6,131.8,61.6,35.8,29.5$, 18.7; HRMS (ESI-TOF): exact mass calcd for $\mathrm{C}_{10} \mathrm{H}_{10} \mathrm{ClN}_{4} \mathrm{O}(\mathrm{M}+\mathrm{H})^{+}$requires $\mathrm{m} / \mathrm{z}$ 237.0538, found $\mathrm{m} / \mathrm{z} 237.0539$.

rac-2-(2-Chloro-6-(dimethylamino)-9H-purin-9-yl)cyclopentan-1-one (11)

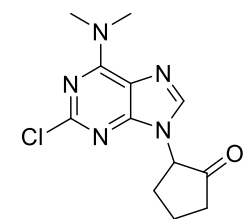

White solid, m.p. $152.8-154.0{ }^{\circ} \mathrm{C} ; 0.96 \mathrm{~g}, 69 \%$ yield; ${ }^{1} \mathbf{H}$ NMR $\left(600 \mathrm{MHz}, \mathrm{CDCl}_{3}\right) \delta 7.59(\mathrm{~s}, 1 \mathrm{H})$, $4.95(\mathrm{dd}, J=12.0,8.4 \mathrm{~Hz}, 1 \mathrm{H}), 3.71(\mathrm{~s}, 3 \mathrm{H}), 3.27(\mathrm{~s}, 3 \mathrm{H}), 2.67-2.62(\mathrm{~m}, 1 \mathrm{H}), 2.57-2.44(\mathrm{~m}, 2 \mathrm{H})$, 2.31-2.23 (m, 2H), 2.05-1.98 (m, 1H). ${ }^{13} \mathbf{C}$ NMR $\left(100 \mathrm{MHz}, \mathrm{CDCl}_{3}\right) \delta$ 211.2, 155.3, 153.8, 151.9, 137.3, 118.9, 60.6, 38.2, 35.7, 29.9, 18.4; HRMS (ESI-TOF): exact mass calcd for $\mathrm{C}_{12} \mathrm{H}_{15} \mathrm{ClN}_{5} \mathrm{O}$ $(\mathrm{M}+\mathrm{H})^{+}$requires $\mathrm{m} / \mathrm{z} 280.0960$, found $\mathrm{m} / \mathrm{z} 280.0962$.

rac-2-(2-Chloro-6-(diethylamino)-9H-purin-9-yl)cyclopentan-1-one (1 m)<smiles>CCn1c(Cl)nc2c1ncn2C1CCCC1=O</smiles> 
Yellow oil, $1.01 \mathrm{~g}, 66 \%$ yield; ${ }^{1} \mathbf{H}$ NMR $\left(600 \mathrm{MHz}, \mathrm{CDCl}_{3}\right) \delta 7.59$ (s, $\left.1 \mathrm{H}\right), 4.95(\mathrm{dd}, J=12.0,8.4$ $\mathrm{Hz}, 1 \mathrm{H}), 4.16(\mathrm{~s}, 2 \mathrm{H}), 3.72(\mathrm{~s}, 2 \mathrm{H}), 2.67-2.45(\mathrm{~m}, 3 \mathrm{H}), 2.31-2.23(\mathrm{~m}, 2 \mathrm{H}), 2.05-1.98(\mathrm{~m}, 1 \mathrm{H})$, 1.26-1.24 (m, 6H). ${ }^{13} \mathrm{C}$ NMR $\left(150 \mathrm{MHz}, \mathrm{CDCl}_{3}\right) \delta 211.3,154.1,153.8,151.9,137.3,118.4,60.5$, 43.7, 42.9, 35.7, 29.9, 18.4, 14.1, 12.8; HRMS (ESI-TOF): exact mass calcd for $\mathrm{C}_{14} \mathrm{H}_{19} \mathrm{ClN}_{5} \mathrm{O}$ $(\mathrm{M}+\mathrm{H})^{+}$requires $\mathrm{m} / \mathrm{z} 308.1273$, found $\mathrm{m} / \mathrm{z} 308.1273$.

rac-2-(2-Chloro-6-(pyrrolidin-1-yl)-9H-purin-9-yl)cyclopentan-1-one (1n)

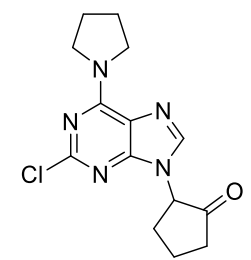

White solid, m.p. $162.9-167.1{ }^{\circ} \mathrm{C} ; 1.07 \mathrm{~g}, 70 \%$ yield; ${ }^{1} \mathbf{H}$ NMR $\left(400 \mathrm{MHz}, \mathrm{CDCl}_{3}\right) \delta 7.58(\mathrm{~s}, 1 \mathrm{H})$, $4.94(\mathrm{dd}, J=12.0,8.0 \mathrm{~Hz}, 1 \mathrm{H}), 4.11(\mathrm{t}, J=6.8 \mathrm{~Hz}, 2 \mathrm{H}), 3.71(\mathrm{t}, J=6.8 \mathrm{~Hz}, 2 \mathrm{H}), 2.67-2.61(\mathrm{~m}$, $1 \mathrm{H}), 2.55-2.44(\mathrm{~m}, 2 \mathrm{H}), 2.33-2.20(\mathrm{~m}, 2 \mathrm{H}), 2.06-1.92(\mathrm{~m}, 5 \mathrm{H}) .{ }^{13} \mathbf{C}$ NMR $\left(100 \mathrm{MHz}, \mathrm{CDCl}_{3}\right) \delta$ 211.2, 154.1, 153.4, 151.5, 137.8, 119.0, 60.5, 48.9, 47.8, 35.7, 29.9, 26.3, 24.2, 18.4; HRMS (ESI-TOF): exact mass calcd for $\mathrm{C}_{14} \mathrm{H}_{17} \mathrm{ClN}_{5} \mathrm{O}(\mathrm{M}+\mathrm{H})^{+}$requires $\mathrm{m} / \mathrm{z} 306.1116$, found $\mathrm{m} / \mathrm{z}$ 306.1123.

rac-2-(2-Chloro-6-morpholino-9H-purin-9-yl)cyclopentan-1-one (10)

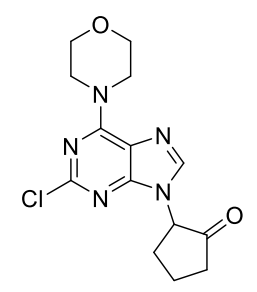

White solid, m.p. $162.0-165.4{ }^{\circ} \mathrm{C} ; 0.96$ g, 60\% yield; ${ }^{1} \mathbf{H}$ NMR $\left(600 \mathrm{MHz}, \mathrm{CDCl}_{3}\right) \delta 7.62(\mathrm{~s}, 1 \mathrm{H})$, $4.97(\mathrm{t}, J=10.2 \mathrm{~Hz}, 1 \mathrm{H}), 4.30(\mathrm{~s}, 4 \mathrm{H}), 3.81(\mathrm{~s}, 4 \mathrm{H}), 2.69-2.65(\mathrm{~m}, 1 \mathrm{H}), 2.59-2.47(\mathrm{~m}, 2 \mathrm{H})$, 2.33-2.56 (m, 2H), 2.07-2.01 (m, 1H). ${ }^{13} \mathbf{C}$ NMR (150 MHz, $\left.\mathrm{CDCl}_{3}\right) \delta 211.1,154.1,153.9,152.4$, 137.6, 118.7, 67.1, 60.7, 35.7, 30.0, 18.5; HRMS (ESI-TOF): exact mass calcd for $\mathrm{C}_{14} \mathrm{H}_{17} \mathrm{ClN}_{5} \mathrm{O}_{2}$ $(\mathrm{M}+\mathrm{H})^{+}$requires $\mathrm{m} / \mathrm{z}$ 322.1065, found $\mathrm{m} / \mathrm{z} 322.1065$.

rac-2-(2-Amino-6-chloro-9H-purin-9-yl)cyclopentan-1-one (1p)<smiles>Nc1nc(Cl)c2ncn(C3CCCC3=O)n12</smiles>

Yellow solid, $184.6-191.4{ }^{\circ} \mathrm{C} ; 0.70 \mathrm{~g}, 56 \%$ yield; ${ }^{1} \mathrm{H}$ NMR (400 MHz, DMSO) $\delta 8.10(\mathrm{~s}, 1 \mathrm{H})$, 
$6.89(\mathrm{~s}, 2 \mathrm{H}), 5.04(\mathrm{dd}, J=12.0,8.8 \mathrm{~Hz}, 1 \mathrm{H}), 2.45-2.35(\mathrm{~m}, 4 \mathrm{H}), 2.14-2.07(\mathrm{~m}, 1 \mathrm{H}), 2.01-1.91(\mathrm{~m}$, 1H). ${ }^{13}$ C NMR (100 MHz, DMSO) $\delta 212.4,159.7,153.9,149.7,142.8,123.4,60.3,35.6,28.3$, 18.2; HRMS (ESI-TOF): exact mass calcd for $\mathrm{C}_{10} \mathrm{H}_{11} \mathrm{ClN}_{5} \mathrm{O}(\mathrm{M}+\mathrm{H})^{+}$requires $\mathrm{m} / \mathrm{z} 252.0647$, found $\mathrm{m} / \mathrm{z} 252.0642$.

rac-2-(2-(Dimethylamino)-9H-purin-9-yl)cyclopentan-1-one (1q)

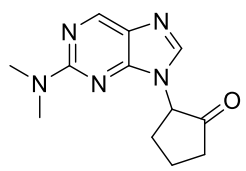

Yellow oil, 0.83 g, 68\% yield; ${ }^{1} \mathbf{H}$ NMR $\left(400 \mathrm{MHz}, \mathrm{CDCl}_{3}\right) \delta 8.71(\mathrm{~s}, 1 \mathrm{H}), 7.63(\mathrm{~s}, 1 \mathrm{H}), 4.74(\mathrm{dd}$, $J=12.0,8.4 \mathrm{~Hz}, 1 \mathrm{H}), 3.17(\mathrm{~s}, 6 \mathrm{H}), 2.63-2.45(\mathrm{~m}, 4 \mathrm{H}), 2.36-2.28(\mathrm{~m}, 1 \mathrm{H}), 2.07-1.97(\mathrm{~m}, 1 \mathrm{H}) .{ }^{13} \mathrm{C}$ NMR $\left(150 \mathrm{MHz}, \mathrm{CDCl}_{3}\right) \delta 211.5,159.7,152.9,149.5,140.9,126.5,60.3,37.7,36.4,3.11,29.2$, 18.9; HRMS (ESI-TOF): exact mass calcd for $\mathrm{C}_{12} \mathrm{H}_{16} \mathrm{~N}_{5} \mathrm{O}(\mathrm{M}+\mathrm{H})^{+}$requires $\mathrm{m} / \mathrm{z} 246.1349$, found $\mathrm{m} / \mathrm{z} 246.1349$.

rac-2-(6-(Dimethylamino)-9H-purin-9-yl)cyclohexan-1-one (1r)

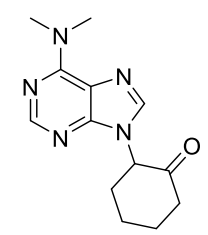

White solid, m.p. $142.6-145.2^{\circ} \mathrm{C} ; 0.56 \mathrm{~g}, 43 \%$ yield; ${ }^{1} \mathbf{H}$ NMR $\left(600 \mathrm{MHz}, \mathrm{CDCl}_{3}\right) \delta 8.25(\mathrm{~s}, 1 \mathrm{H})$, $7.75(\mathrm{~s}, 1 \mathrm{H}), 5.34(\mathrm{dd}, J=13.2,6.0 \mathrm{~Hz}, 1 \mathrm{H}), 3.49(\mathrm{~s}, 6 \mathrm{H}), 2.63-2.59(\mathrm{~m}, 1 \mathrm{H}), 2.57-2.51(\mathrm{~m}, 1 \mathrm{H})$, 2.48-2.44 (m, 1H), 2.22-2.17 (m, 1H), 2.16-2.09 (m, 1H), 2.09-2.04 (m, 1H), 1.96-1.88 (m, 1H), $1.82-1.74(\mathrm{~m}, 1 \mathrm{H}) .{ }^{13} \mathrm{C}$ NMR $\left(150 \mathrm{MHz}, \mathrm{CDCl}_{3}\right) \delta 204.2,155.0,152.2,150.3,137.4,119.6,61.0$, 41.4, 38.6, 35.1, 27.3, 24.9; HRMS (ESI-TOF): exact mass calcd for $\mathrm{C}_{13} \mathrm{H}_{18} \mathrm{~N}_{5} \mathrm{O}(\mathrm{M}+\mathrm{H})^{+}$requires $\mathrm{m} / \mathrm{z} 260.1506$, found $\mathrm{m} / \mathrm{z} 260.1514$.

rac-2-(6-(Dimethylamino)-9H-purin-9-yl)cycloheptan-1-one (1s)

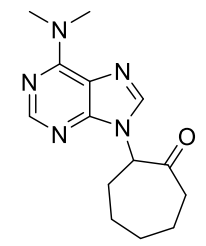

White solid, m.p. $157.8-158.3{ }^{\circ} \mathrm{C} ; 0.63 \mathrm{~g}, 46 \%$ yield; ${ }^{1} \mathbf{H}$ NMR $\left(400 \mathrm{MHz}, \mathrm{CDCl}_{3}\right) \delta 8.26(\mathrm{~s}, 1 \mathrm{H})$, $7.82(\mathrm{~s}, 1 \mathrm{H}), 5.50(\mathrm{dd}, J=11.2,3.2 \mathrm{~Hz}, 1 \mathrm{H}), 3.50(\mathrm{~s}, 6 \mathrm{H}), 2.79-2.76(\mathrm{~m}, 1 \mathrm{H}), 2.60-2.53(\mathrm{~m}, 1 \mathrm{H})$, 
2.22-1.75 (m, 7H), 1.42-1.36 (m, 1H). ${ }^{13} \mathbf{C}$ NMR $\left(100 \mathrm{MHz}, \mathrm{CDCl}_{3}\right) \delta$ 207.1, 154.9, 152.2, 149.9, 137.7, 119.4, 61.8, 41.9, 38.6, 32.6, 28.6, 28.2, 23.0; HRMS (ESI-TOF): exact mass calcd for $\mathrm{C}_{14} \mathrm{H}_{20} \mathrm{~N}_{5} \mathrm{O}(\mathrm{M}+\mathrm{H})^{+}$requires $\mathrm{m} / \mathrm{z} 274.1662$, found $\mathrm{m} / \mathrm{z} 274.1669$.

rac-2-(6-(Dimethylamino)-9H-purin-9-yl)-2,3-dihydro-1H-inden-1-one (1t)

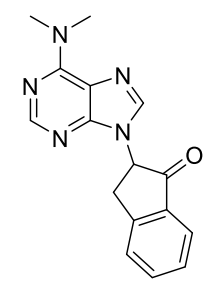

Yellow oil, $0.62 \mathrm{~g}, 42 \%$ yield; ${ }^{1} \mathbf{H}$ NMR $\left(600 \mathrm{MHz}, \mathrm{CDCl}_{3}\right) \delta 8.24(\mathrm{~s}, 1 \mathrm{H}), 7.87(\mathrm{~d}, J=7.8 \mathrm{~Hz}$, 1H), $7.74(\mathrm{~s}, 1 \mathrm{H}), 7.70(\mathrm{t}, J=7.8 \mathrm{~Hz}, 1 \mathrm{H}), 7.52(\mathrm{~d}, J=7.8 \mathrm{~Hz}, 1 \mathrm{H}), 7.47$ (t, $J=7.8 \mathrm{~Hz}, 1 \mathrm{H}), 5.36$ $(\mathrm{dd}, J=8.4,5.4 \mathrm{~Hz}, 1 \mathrm{H}), 3.83(\mathrm{dd}, J=16.8,8.4 \mathrm{~Hz}, 1 \mathrm{H}), 3.59-3.51(\mathrm{~m}, 7 \mathrm{H}) .{ }^{13} \mathbf{C}$ NMR $(100 \mathrm{MHz}$, $\left.\mathrm{CDCl}_{3}\right) \delta 199.6,155.0,152.5,150.7,150.6,137.5,136.3,134.5,128.5,126.8,124.9,120.1,58.9$, 38.6, 33.9; HRMS (ESI-TOF): exact mass calcd for $\mathrm{C}_{16} \mathrm{H}_{16} \mathrm{~N}_{5} \mathrm{O}(\mathrm{M}+\mathrm{H})^{+}$requires m/z 294.1349, found $\mathrm{m} / \mathrm{z} 294.1348$.

rac-2-(1H-Benzo[d] imidazol-1-yl)cyclopentan-1-one (3a)

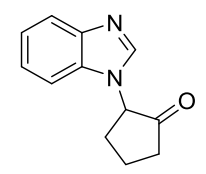

Yellow oil, $0.56 \mathrm{~g}, 56 \%$ yield; ${ }^{1} \mathbf{H}$ NMR $\left(600 \mathrm{MHz}, \mathrm{CDCl}_{3}\right) \delta$ 7.90-7.85 (m, 2H), 7.32-7.26 (m, $3 \mathrm{H}), 4.83(\mathrm{t}, J=10.2 \mathrm{~Hz}, 1 \mathrm{H}), 2.69-2.61(\mathrm{~m}, 2 \mathrm{H}), 2.51-2.45(\mathrm{~m}, 1 \mathrm{H}), 2.29$ (s, 2H), 2.09-2.04 (m, 1H). ${ }^{13} \mathbf{C}$ NMR (150 MHz, $\left.\mathrm{CDCl}_{3}\right) \delta 211.1,143.8,141.9,133.3,123.2,122.5,120.7,109.9,61.9$, 36.0, 29.7, 18.4; HRMS (ESI-TOF): exact mass calcd for $\mathrm{C}_{12} \mathrm{H}_{13} \mathrm{~N}_{2} \mathrm{O}(\mathrm{M}+\mathrm{H})^{+}$requires $\mathrm{m} / \mathrm{z}$ 201.1022, found $\mathrm{m} / \mathrm{z} 201.1022$.

rac-2-(1H-Benzo[d][1,2,3]triazol-1-yl)cyclopentan-1-one (3b)

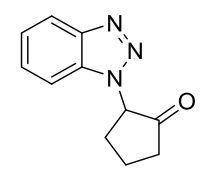

Yellow solid, m.p. $74.1-76.5{ }^{\circ} \mathrm{C} ; 0.47 \mathrm{~g}, 47 \%$ yield; ${ }^{1} \mathbf{H}$ NMR $\left(600 \mathrm{MHz}, \mathrm{CDCl}_{3}\right) \delta 8.07(\mathrm{~d}, J=8.4$ $\mathrm{Hz}, 1 \mathrm{H}), 7.47$ (t, $J=7.8 \mathrm{~Hz}, 1 \mathrm{H}), 7.41(\mathrm{~d}, J=7.8 \mathrm{~Hz}, 1 \mathrm{H}), 7.37$ (t, $J=7.8 \mathrm{~Hz}, 1 \mathrm{H}), 5.26$ (dd, $J=$ 10.8, 8.4 Hz, 1H), 2.79-2.71 (m, 2H), 2.64-2.52 (m, 2H), 2.42-2.36 (m, 1H), 2.16-2.09 (m, 1H). 
${ }^{13} \mathrm{C}$ NMR $\left(150 \mathrm{MHz}, \mathrm{CDCl}_{3}\right) \delta 210.3,146.1,133.1,127.7,124.2,120.4,109.6,64.1,36.3,29.2$, 19.0; HRMS (ESI-TOF): exact mass calcd for $\mathrm{C}_{11} \mathrm{H}_{12} \mathrm{~N}_{3} \mathrm{O}(\mathrm{M}+\mathrm{H})^{+}$requires $\mathrm{m} / \mathrm{z} 202.0975$, found $\mathrm{m} / \mathrm{z} 202.0975$.

rac-2-(1H-Imidazo[4,5-c]pyridin-1-yl)cyclopentan-1-one (3c)

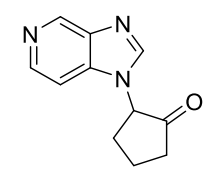

Yellow oil, $0.11 \mathrm{~g}, 11 \%$ yield; ${ }^{1} \mathbf{H}$ NMR $\left(400 \mathrm{MHz}, \mathrm{CDCl}_{3}\right) \delta 8.71(\mathrm{~s}, 1 \mathrm{H}), 8.46(\mathrm{~d}, J=5.6 \mathrm{~Hz}$, 1H), $8.01(\mathrm{~s}, 1 \mathrm{H}), 7.72(\mathrm{~d}, J=5.6 \mathrm{~Hz}, 1 \mathrm{H}), 4.91(\mathrm{dd}, J=12.0,8.4 \mathrm{~Hz}, 1 \mathrm{H}), 2.79-2.73(\mathrm{~m}, 1 \mathrm{H})$, 2.69-2.62 (m, 1H), 2.54-2.44 (m, 1H), 2.38-2.27 (m, 2H), 2.15-2.07 (m, 1H). ${ }^{13}$ C NMR (150 MHz, $\left.\mathrm{CDCl}_{3}\right) \delta 210.2,149.0,144.6,142.3,133.6,131.3,115.5,62.4,35.9,30.0,18.4$; HRMS (ESI-TOF): exact mass calcd for $\mathrm{C}_{11} \mathrm{H}_{12} \mathrm{~N}_{3} \mathrm{O}(\mathrm{M}+\mathrm{H})^{+}$requires $\mathrm{m} / \mathrm{z}$ 202.0975, found $\mathrm{m} / \mathrm{z} 202.0974$. rac-2-(7H-Purin-7-yl)cyclopentan-1-one (3d)

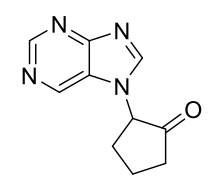

White oil, 0.26 g, 26\% yield; ${ }^{1} \mathbf{H}$ NMR $\left(600 \mathrm{MHz}, \mathrm{CDCl}_{3}\right) \delta 9.15(\mathrm{~s}, 1 \mathrm{H}), 8.84(\mathrm{~s}, 1 \mathrm{H}), 8.27(\mathrm{~s}$, $1 \mathrm{H}), 4.97-4.93(\mathrm{~m}, 1 \mathrm{H}), 2.84-2.79(\mathrm{~m}, 1 \mathrm{H}), 2.71-2.66(\mathrm{~m}, 1 \mathrm{H}), 2.55-2.48(\mathrm{~m}, 1 \mathrm{H}), 2.41-2.34(\mathrm{~m}$, 2H), 2.19-2.13 (m, 1H). ${ }^{13} \mathbf{C}$ NMR (150 MHz, $\left.\mathrm{CDCl}_{3}\right) \delta$ 209.6, 160.9, 153.6, 146.9, 140.6, 125.0, 62.9, 35.7, 29.7, 18.5; HRMS (ESI-TOF): exact mass calcd for $\mathrm{C}_{10} \mathrm{H}_{11} \mathrm{~N}_{4} \mathrm{O}(\mathrm{M}+\mathrm{H})^{+}$requires $\mathrm{m} / \mathrm{z}$ 203.0927, found $\mathrm{m} / \mathrm{z} 203.0931$.

rac-2-(3H-Imidazo[4,5-b]pyridin-3-yl)cyclopentan-1-one (3e)

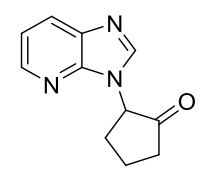

Yellow oil, $0.23 \mathrm{~g}, 23 \%$ yield; ${ }^{1} \mathbf{H}$ NMR $\left(600 \mathrm{MHz}, \mathrm{CDCl}_{3}\right) \delta 8.28(\mathrm{~d}, J=4.8 \mathrm{~Hz}, 1 \mathrm{H}), 8.02(\mathrm{~d}, J=$ $8.4 \mathrm{~Hz}, 1 \mathrm{H}), 7.98$ (s, 1H), 7.17 (dd, $J=8.4,4.8 \mathrm{~Hz}, 1 \mathrm{H}), 5.00$ (dd, $J=12.6,8.4 \mathrm{~Hz}, 1 \mathrm{H}), 2.61-2.57$ (m, 1H), 2.53-2.50 (m, 2H), 2.47-2.39(m, 1H), 2.25-2.20 (m, 1H), 2.01-1.92 (m, 1H). ${ }^{13} \mathbf{C}$ NMR $\left(150 \mathrm{MHz}, \mathrm{CDCl}_{3}\right) \delta 211.6,146.5,144.0,143.3,135.2,127.9,118.4,60.7,35.8,29.4,18.4$; HRMS (ESI-TOF): exact mass calcd for $\mathrm{C}_{11} \mathrm{H}_{12} \mathrm{~N}_{3} \mathrm{O}(\mathrm{M}+\mathrm{H})^{+}$requires $\mathrm{m} / \mathrm{z} 202.0975$, found $\mathrm{m} / \mathrm{z}$ 202.0975 . 
rac-2-(9H-Purin-9-yl)cyclopentan-1-one (3f)

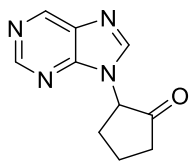

Yellow solid, m.p. $145.8-147.9{ }^{\circ} \mathrm{C} ; 0.46$ g, 46\% yield; ${ }^{1} \mathbf{H}$ NMR $\left(600 \mathrm{MHz}, \mathrm{CDCl}_{3}\right) \delta 9.14(\mathrm{~s}, 1 \mathrm{H})$, $8.92(\mathrm{~s}, 1 \mathrm{H}), 8.05(\mathrm{~s}, 1 \mathrm{H}), 5.01(\mathrm{dd}, J=12.6,8.4 \mathrm{~Hz}, 1 \mathrm{H}), 2.72-2.67(\mathrm{~m}, 1 \mathrm{H}), 2.63-2.55(\mathrm{~m}, 2 \mathrm{H})$, 2.54-2.46 (m, 1H), 2.37-2.32 (m, 1H), 2.11-2.02 (m, 1H). ${ }^{13} \mathbf{C}$ NMR $\left(150 \mathrm{MHz}, \mathrm{CDCl}_{3}\right) \delta 210.7$, 152.6, 151.3, 148.9, 144.5, 134.2, 61.1, 35.9, 29.6, 18.7; HRMS (ESI-TOF): exact mass calcd for $\mathrm{C}_{10} \mathrm{H}_{11} \mathrm{~N}_{4} \mathrm{O}(\mathrm{M}+\mathrm{H})^{+}$requires $\mathrm{m} / \mathrm{z} 203.0927$, found $\mathrm{m} / \mathrm{z} 203.0926$.

rac-1,3-Dimethyl-9-(2-oxocyclopentyl)-3,9-dihydro-1H-purine-2,6-dione (3g)

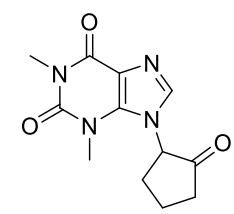

White solid, m.p. 180.2-182.5 ${ }^{\circ} \mathrm{C} ; 0.75$ g, 57\% yield; ${ }^{1} \mathbf{H}$ NMR $\left(600 \mathrm{MHz}, \mathrm{CDCl}_{3}\right) \delta 7.56(\mathrm{~s}, 1 \mathrm{H})$, $4.79(\mathrm{dd}, J=12.6,8.4 \mathrm{~Hz}, 1 \mathrm{H}), 3.56(\mathrm{~s}, 3 \mathrm{H}), 3.33(\mathrm{~s}, 3 \mathrm{H}), 2.76-2.69(\mathrm{~m}, 1 \mathrm{H}), 2.56-2.52(\mathrm{~m}, 1 \mathrm{H})$, 2.49-2.42 (m, 2H), 2.30-2.25 (m, 1H), 1.99-1.90 (m, 1H). ${ }^{13} \mathbf{C}$ NMR (150 MHz, $\left.\mathrm{CDCl}_{3}\right) \delta 210.6$, 155.0, 151.6, 149.3, 141.7, 106.4, 63.9, 35.1, 30.6, 29.9, 28.2, 18.4; HRMS (ESI-TOF): exact mass calcd for $\mathrm{C}_{12} \mathrm{H}_{14} \mathrm{~N}_{4} \mathrm{NaO}_{3}(\mathrm{M}+\mathrm{Na})^{+}$requires $\mathrm{m} / \mathrm{z} 285.0958$, found $\mathrm{m} / \mathrm{z} 285.0958$.

\section{2-Allyl-2-(6-methoxy-9H-purin-9-yl)cyclopentan-1-one (3h)}

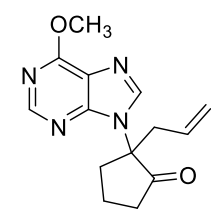

White oil, $84.4 \mathrm{mg}, 31 \%$ yield; ${ }^{1} \mathbf{H}$ NMR $\left(600 \mathrm{MHz}, \mathrm{CDCl}_{3}\right) \delta 8.47(\mathrm{~s}, 1 \mathrm{H}), 8.21(\mathrm{~s}, 1 \mathrm{H})$, 5.75-5.68 (m, 1H), 5.16-5.10 (m, 2H), $4.17(\mathrm{~s}, 3 \mathrm{H}), 2.95-2.89(\mathrm{~m}, 3 \mathrm{H}), 2.74-2.64(\mathrm{~m}, 2 \mathrm{H}), 2.56-2.50(\mathrm{~m}, 1 \mathrm{H})$, 2.20-2.13 (m, 1H), 2.06-1.99 (m, 1H); $\left.{ }^{13} \mathbf{C ~ N M R ~ ( 1 5 0 ~ M H z , ~} \mathrm{CDCl}_{3}\right) \delta 213.1,161.3,151.6,140.9$, 130.2, 121.2, 68.2, 54.3, 38.8, 36.6, 34.4, 18.3; HRMS (ESI-TOF): exact mass calcd for $\mathrm{C}_{14} \mathrm{H}_{17} \mathrm{~N}_{4} \mathrm{O}_{2}(\mathrm{M}+\mathrm{H})^{+}$requires $\mathrm{m} / \mathrm{z} 273.1346$, found $\mathrm{m} / \mathrm{z} 273.1345$.

2-(6-Methoxy-9H-purin-9-yl)cyclopent-1-en-1-yl acetate (3i)<smiles>COc1ncnc2c1ncn2C1=C(OC(C)=O)CCC1</smiles> 
White solid, m.p. $105.1-109.3{ }^{\circ} \mathrm{C} ; 0.15 \mathrm{~g}, 53 \%$ yield; TLC: $\mathrm{R}_{\mathrm{f}}=0.46$ (petroleum:ethyl acetate $=$ 1:1) [UV]; ${ }^{1} \mathrm{H}$ NMR $\left(400 \mathrm{MHz}, \mathrm{CDCl}_{3}\right) \delta 8.54$ (s, 1H), 7.99 (s, 1H), 4.19 (s, 3H), 3.06-3.01 (m, 2H), 2.81-2.76 (m, 2H), 2.22-2.15 (m, 2H), $2.11(\mathrm{~s}, 3 \mathrm{H}) ;{ }^{13} \mathbf{C}$ NMR (100 MHz, $\left.\mathrm{CDCl}_{3}\right) \delta$ 167.9, 161.4, 152.5, 151.8, 142.6, 141.2, 121.3, 119.2, 54.4, 30.0, 29.8, 20.9, 18.8; HRMS (ESI-TOF): exact mass calcd for $\mathrm{C}_{13} \mathrm{H}_{15} \mathrm{~N}_{4} \mathrm{O}_{3}(\mathrm{M}+\mathrm{H})^{+}$requires $\mathrm{m} / \mathrm{z} 275.1139$, found $\mathrm{m} / \mathrm{z} 275.1132$.

\section{(2) The analytical and spectral characterization data of the products}

\section{$(1 R, 2 S)-2-(6-M e t h o x y-9 H-p u r i n-9-y l) c y c l o p e n t a n-1-o l ~(2 a)$}

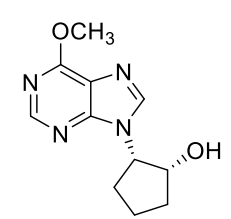

White solid, m.p. $152.5-156.0{ }^{\circ} \mathrm{C} .22 .4 \mathrm{mg}, 96 \%$ yield, $97 \%$ ee; $[\alpha]_{\mathrm{D}^{20}}=-78.90(\mathrm{c}=0.218$, $\mathrm{CH}_{2} \mathrm{Cl}_{2}$ ); HPLC CHIRALCEL ID, $n$-hexane $/ 2$-propanol $=80 / 20$, flow rate $=0.7 \mathrm{~mL} / \mathrm{min}, \lambda=250$ $\mathrm{nm}$, retention time: $23.893 \mathrm{~min}, 25.767 \mathrm{~min}$; TLC: $\mathrm{R}_{\mathrm{f}}=0.31$ (dichloromethane:methanol $=30: 1$ ) [UV]; ${ }^{1} \mathrm{H}$ NMR $\left(600 \mathrm{MHz}, \mathrm{CD}_{3} \mathrm{OD}\right) \delta 8.49(\mathrm{~s}, 1 \mathrm{H}), 8.38(\mathrm{~s}, 1 \mathrm{H}), 4.91-4.87(\mathrm{~m}, 1 \mathrm{H}), 4.36-4.34(\mathrm{~m}$, 1H), 4.17 (s, 3H), 2.41-2.34 (m, 1H), 2.27-2.22 (m, 1H), 2.15-2.04 (m, 2H), 1.90-1.84 (m, 1H), 1.83-1.77 (m, 1H); ${ }^{13} \mathbf{C}$ NMR (150 MHz, $\left.\mathrm{CDCl}_{3}\right) \delta 159.8,151.5,151.4,142.2,119.9,71.3,59.7$, 54.1, 32.3, 27.7, 20.3; HRMS (ESI-TOF): exact mass calcd for $\mathrm{C}_{11} \mathrm{H}_{15} \mathrm{~N}_{4} \mathrm{O}_{2}(\mathrm{M}+\mathrm{H})^{+}$requires $\mathrm{m} / \mathrm{z}$ 235.1190, found $\mathrm{m} / \mathrm{z} 235.1190$.

(1R,2S)-2-(6-Ethoxy-9H-purin-9-yl)cyclopentan-1-ol (2b)

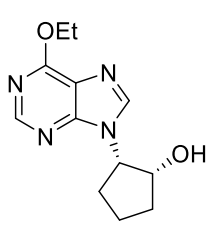

White solid, m.p. $176.3-178.5{ }^{\circ} \mathrm{C} .23 .8 \mathrm{mg}, 96 \%$ yield, $90 \%$ ee; $[\alpha]_{\mathrm{D}^{20}}=-80.30(\mathrm{c}=0.594$, $\mathrm{CH}_{2} \mathrm{Cl}_{2}$ ); HPLC CHIRALCEL AS-H, $n$-hexane $/ 2$-propanol $=90 / 10$, flow rate $=0.8 \mathrm{~mL} / \mathrm{min}, \lambda=$ $256 \mathrm{~nm}$, retention time: $9.870 \mathrm{~min}, 25.590 \mathrm{~min} ; \mathbf{T L C}: \mathrm{R}_{\mathrm{f}}=0.34$ (dichloromethane: methanol $=$ 30:1) [UV]; ${ }^{1} \mathbf{H}$ NMR (600 MHz, CD $\left.{ }_{3} \mathrm{OD}\right) \delta 8.46(\mathrm{~s}, 1 \mathrm{H}), 8.37(\mathrm{~s}, 1 \mathrm{H}), 4.90-4.87(\mathrm{~m}, 1 \mathrm{H})$, 4.67-4.63 (m, 2H), 4.36-4.34 (m, 1H), 2.39-2.35 (m, 1H), 2.26-2.21 (m, 1H), 2.15-2.04 (m, 2H), $1.89-1.87(\mathrm{~m}, 1 \mathrm{H}), 1.80-1.77(\mathrm{~m}, 1 \mathrm{H}), 1.49(\mathrm{t}, J=7.2 \mathrm{~Hz}, 3 \mathrm{H}) ;{ }^{13} \mathbf{C}$ NMR $\left(100 \mathrm{MHz}, \mathrm{CDCl}_{3}\right) \delta$ 159.3, 151.4, 151.2, 141.9, 119.6, 71.1, 63.1, 59.5, 32.1, 27.6, 20.3, 14.5; HRMS (ESI-TOF): exact mass calcd for $\mathrm{C}_{12} \mathrm{H}_{17} \mathrm{~N}_{4} \mathrm{O}_{2}(\mathrm{M}+\mathrm{H})^{+}$requires $\mathrm{m} / \mathrm{z} 249.1346$, found $\mathrm{m} / \mathrm{z} 249.1345$. 
(1R,2S)-2-(6-(Propylthio)-9H-purin-9-yl)cyclopentan-1-ol (2c)

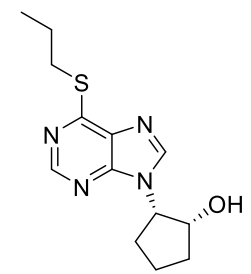

White solid, m.p. $119.3-120.9{ }^{\circ} \mathrm{C} .24 .5 \mathrm{mg}, 88 \%$ yield, $86 \%$ ee; $[\alpha]_{\mathrm{D}}{ }^{20}=-44.02(\mathrm{c}=0.234$, $\mathrm{CH}_{2} \mathrm{Cl}_{2}$ ); HPLC CHIRALCEL IA, $n$-hexane $/ 2$-propanol $=90 / 10$, flow rate $=0.7 \mathrm{~mL} / \mathrm{min}, \lambda=250$ $\mathrm{nm}$, retention time: $14.080 \mathrm{~min}, 15.787 \mathrm{~min}$; TLC: $\mathrm{R}_{\mathrm{f}}=0.36$ (dichloromethane:methanol $=30: 1$ ) [UV]; ${ }^{1} \mathrm{H}$ NMR $\left(600 \mathrm{MHz}, \mathrm{CD}_{3} \mathrm{OD}\right) \delta 8.66(\mathrm{~s}, 1 \mathrm{H}), 8.42(\mathrm{~s}, 1 \mathrm{H}), 4.91-4.88(\mathrm{~m}, 1 \mathrm{H}), 4.35-4.33(\mathrm{~m}$, $1 \mathrm{H}), 3.39-3.36(\mathrm{~m}, 2 \mathrm{H}), 2.41-2.34(\mathrm{~m}, 1 \mathrm{H}), 2.27-2.22(\mathrm{~m}, 1 \mathrm{H}), 2.15-2.04(\mathrm{~m}, 2 \mathrm{H}), 1.89-1.84(\mathrm{~m}$, 1H), 1.82-1.78 (m, 3H), $1.08(\mathrm{t}, J=7.2 \mathrm{~Hz}, 3 \mathrm{H}) ;{ }^{13} \mathbf{C}$ NMR $\left(100 \mathrm{MHz}, \mathrm{CDCl}_{3}\right) \delta 160.6,151.5$, 148.1, 142.6, 130.2, 71.7, 59.4, 32.4, 30.8, 27.9, 22.9, 20.3, 13.7; HRMS (ESI-TOF): exact mass calcd for $\mathrm{C}_{13} \mathrm{H}_{19} \mathrm{~N}_{4} \mathrm{OS}(\mathrm{M}+\mathrm{H})^{+}$requires $\mathrm{m} / \mathrm{z}$ 279.1274, found m/z 279.1271 .

(1R,2S)-2-(6-Phenyl-9H-purin-9-yl)cyclopentan-1-ol (2d)

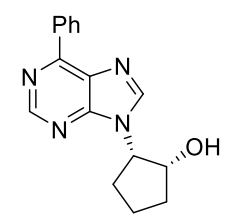

Yellow oil, $26.1 \mathrm{mg}, 93 \%$ yield, $85 \%$ ee; $[\alpha]_{\mathrm{D}}{ }^{20}=-32.41\left(\mathrm{c}=0.254, \mathrm{CH}_{2} \mathrm{Cl}_{2}\right) ;$ HPLC CHIRALCEL ID, $n$-hexane $/ 2$-propanol $=80 / 20$, flow rate $=0.7 \mathrm{~mL} / \mathrm{min}, \lambda=250 \mathrm{~nm}$, retention time: $14.174 \mathrm{~min}, 22.521 \mathrm{~min}$; TLC: $\mathrm{R}_{\mathrm{f}}=0.40$ (dichloromethane:methanol $=30: 1$ ) $[\mathrm{UV}] ;{ }^{1} \mathbf{H}$ NMR $\left(600 \mathrm{MHz}, \mathrm{CD}_{3} \mathrm{OD}\right) \delta 8.92(\mathrm{~s}, 1 \mathrm{H}), 8.63-8.59(\mathrm{~m}, 3 \mathrm{H}), 7.59-7.55(\mathrm{~m}, 3 \mathrm{H}), 5.00-4.96(\mathrm{~m}$, $1 \mathrm{H}), 4.40-4.38(\mathrm{~m}, 1 \mathrm{H}), 2.46-2.39(\mathrm{~m}, 1 \mathrm{H}), 2.30-2.25(\mathrm{~m}, 1 \mathrm{H}), 2.18-2.07(\mathrm{~m}, 2 \mathrm{H}), 1.92-1.79(\mathrm{~m}$, 2H); ${ }^{13} \mathrm{C}$ NMR (100 MHz, $\left.\mathrm{CDCl}_{3}\right) \delta 154.3,152.2,152.0,144.4,135.1,131.1,130.0,129.8,128.7$, 71.3, 59.2, 32.4, 27.6, 20.3; HRMS (ESI-TOF): exact mass calcd for $\mathrm{C}_{16} \mathrm{H}_{17} \mathrm{~N}_{4} \mathrm{O}(\mathrm{M}+\mathrm{H})^{+}$requires $\mathrm{m} / \mathrm{z} 281.1397$, found $\mathrm{m} / \mathrm{z} 281.1396$.

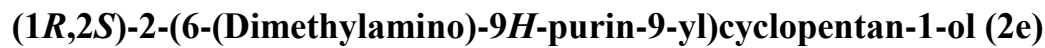

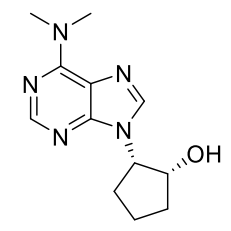

White solid, m.p. $59.7-66.8^{\circ} \mathrm{C} .24 .0 \mathrm{mg}, 97 \%$ yield, $>99 \%$ ee; $[\alpha]_{\mathrm{D}}{ }^{20}=-48.8\left(\mathrm{c}=0.532, \mathrm{CH}_{2} \mathrm{Cl}_{2}\right)$; 
HPLC CHIRALCEL ID, $n$-hexane $/ 2$-propanol $=25 / 75$, flow rate $=0.3 \mathrm{~mL} / \mathrm{min}, \lambda=250 \mathrm{~nm}$, retention time: $18.723 \mathrm{~min}$; TLC: $\mathrm{R}_{\mathrm{f}}=0.38$ (dichloromethane:methanol $=30: 1$ ) [UV]; ${ }^{1} \mathbf{H}$ NMR $\left(400 \mathrm{MHz}, \mathrm{CDCl}_{3}\right) \delta 8.13(\mathrm{~s}, 1 \mathrm{H}), 7.78(\mathrm{~s}, 1 \mathrm{H}), 4.66-4.60(\mathrm{~m}, 1 \mathrm{H}), 4.53-4.50(\mathrm{~m}, 1 \mathrm{H}), 3.32(\mathrm{~s}, 6 \mathrm{H})$, 2.36-2.25 (m, 1H), 2.14-2.02 (m, 3H), 1.93-1.85 (m, 1H), 1.76-1.68 (m, 1H); ${ }^{13} \mathbf{C}$ NMR (100 MHz, $\left.\mathrm{CDCl}_{3}\right) \delta 154.2,151.9,150.2,138.2,119.6,71.7,59.6,38.6,32.4,27.4,20.2$; HRMS (ESI-TOF): exact mass calcd for $\mathrm{C}_{12} \mathrm{H}_{18} \mathrm{~N}_{5} \mathrm{O}(\mathrm{M}+\mathrm{H})^{+}$requires $\mathrm{m} / \mathrm{z} 248.1506$, found $\mathrm{m} / \mathrm{z} 248.1512$.

(1R,2S)-2-(6-(Diethylamino)-9H-purin-9-yl)cyclopentan-1-ol (2f)

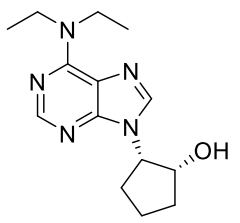

Yellow oil, $25.6 \mathrm{mg}, 93 \%$ yield, $94 \%$ ee; $[\alpha]_{\mathrm{D}}^{20}=-39.41\left(\mathrm{c}=0.444, \mathrm{CH}_{2} \mathrm{Cl}_{2}\right) ;$ HPLC CHIRALCEL ID, $n$-hexane $/ 2$-propanol $=80 / 20$, flow rate $=0.7 \mathrm{~mL} / \mathrm{min}, \lambda=250 \mathrm{~nm}$, retention time: $11.459 \mathrm{~min}, 12.589 \mathrm{~min}$; TLC: $\mathrm{R}_{\mathrm{f}}=0.29$ (dichloromethane:methanol $=30: 1$ ) $[\mathrm{UV}] ;{ }^{1} \mathbf{H}$ NMR $\left(400 \mathrm{MHz}, \mathrm{CDCl}_{3}\right) \delta 8.22(\mathrm{~s}, 1 \mathrm{H}), 7.83(\mathrm{~s}, 1 \mathrm{H}), 4.68-4.62(\mathrm{~m}, 1 \mathrm{H}), 4.50-4.47(\mathrm{~m}, 1 \mathrm{H}), 3.87$ (s, 4H), 2.35-2.25 (m, 1H), 2.18-2.00 (m, 3H), 1.92-1.83 (m, 1H), 1.78-1.67 (m, 1H), $1.24(\mathrm{t}, J=$ $7.2 \mathrm{~Hz}, 6 \mathrm{H}) ;{ }^{13} \mathbf{C}$ NMR $\left(100 \mathrm{MHz}, \mathrm{CDCl}_{3}\right) \delta 153.8,152.2,150.5,138.5,119.5,72.3,59.7,43.2$, 32.6, 27.9, 20.1, 13.6; HRMS (ESI-TOF): exact mass calcd for $\mathrm{C}_{14} \mathrm{H}_{22} \mathrm{~N}_{5} \mathrm{O}(\mathrm{M}+\mathrm{H})^{+}$requires $\mathrm{m} / \mathrm{z}$ 276.1819, found $\mathrm{m} / \mathrm{z} 276.1819$.

(1R,2S)-2-(6-(Pyrrolidin-1-yl)-9H-purin-9-yl)cyclopentan-1-ol (2g)

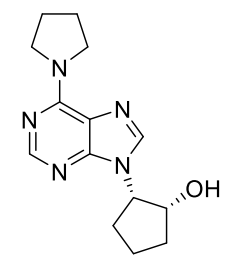

White solid, m.p. $199.8-202.0{ }^{\circ} \mathrm{C} .25 .4 \mathrm{mg}, 93 \%$ yield, $98 \%$ ee; $[\alpha]_{\mathrm{D}^{20}}=-55.50(\mathrm{c}=0.418$, $\mathrm{CH}_{2} \mathrm{Cl}_{2}$ ); HPLC CHIRALCEL IA, $n$-hexane $/ 2$-propanol $=80 / 20$, flow rate $=0.7 \mathrm{~mL} / \mathrm{min}, \lambda=250$ $\mathrm{nm}$, retention time: $15.463 \mathrm{~min}, 19.228 \mathrm{~min} ; \mathbf{T L C}: \mathrm{R}_{\mathrm{f}}=0.31$ (dichloromethane:methanol $=30: 1$ ) $[\mathrm{UV}] ;{ }^{1} \mathrm{H}$ NMR $\left(600 \mathrm{MHz}, \mathrm{CDCl}_{3}\right) \delta 8.11(\mathrm{~s}, 1 \mathrm{H}), 7.77(\mathrm{~s}, 1 \mathrm{H}), 4.63-4.59(\mathrm{~m}, 1 \mathrm{H}), 4.57-4.56(\mathrm{~m}$, 1H), 3.95-3.84 (m, 2H), $3.53(\mathrm{~s}, 2 \mathrm{H}), 2.37-2.30(\mathrm{~m}, 1 \mathrm{H}), 2.16-2.03(\mathrm{~m}, 4 \mathrm{H}), 1.98-1.89(\mathrm{~m}, 4 \mathrm{H})$, 1.76-1.68 (m, 1H); ${ }^{13} \mathbf{C}$ NMR (100 MHz, $\left.\mathrm{CDCl}_{3}\right) \delta 154.2,151.9,150.2,138.2,119.6,71.7,59.6$, 38.6, 32.4, 31.0, 29.8, 27.4, 20.2; HRMS (ESI-TOF): exact mass calcd for $\mathrm{C}_{14} \mathrm{H}_{20} \mathrm{~N}_{5} \mathrm{O}(\mathrm{M}+\mathrm{H})^{+}$ requires $\mathrm{m} / \mathrm{z} 274.1662$, found $\mathrm{m} / \mathrm{z} 274.1663$. 


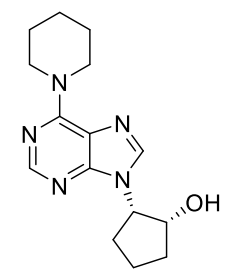

Yellow oil, $26.7 \mathrm{mg}, 93 \%$ yield, $95 \%$ ee; $[\alpha]_{\mathrm{D}}^{20}=-42.92\left(\mathrm{c}=0.226, \mathrm{CH}_{2} \mathrm{Cl}_{2}\right)$; HPLC CHIRALCEL IA, $n$-hexane $/ 2$-propanol $=90 / 10$, flow rate $=0.8 \mathrm{~mL} / \mathrm{min}, \lambda=256 \mathrm{~nm}$, retention time: $17.827 \mathrm{~min}, 20.950 \mathrm{~min}$; TLC: $\mathrm{R}_{\mathrm{f}}=0.29$ (dichloromethane:methanol $=30: 1$ ) $[\mathrm{UV}] ;{ }^{1} \mathbf{H}$ NMR $\left(600 \mathrm{MHz}, \mathrm{CDCl}_{3}\right) \delta 8.19(\mathrm{~s}, 1 \mathrm{H}), 7.83(\mathrm{~s}, 1 \mathrm{H}), 4.67-4.63(\mathrm{~m}, 1 \mathrm{H}), 4.52-4.51(\mathrm{~m}, 1 \mathrm{H})$, 4.12-4.11 (m, 4H), 3.95-3.947 (m, 1H), 2.34-2.28 (m, 1H), 2.15-2.03 (m, 3H), 1.92-1.88 (m, 1H), 1.75-1.69 (m, 3H), 1.66-1.63 (m, 4H); ${ }^{13} \mathbf{C}$ NMR (100 MHz, CDCl $) \delta 153.7,152.1,150.7,138.1$, 119.7, 72.1, 59.7, 46.4, 32.6, 31.3, 27.8, 26.2, 24.9, 20.2; HRMS (ESI-TOF): exact mass calcd for $\mathrm{C}_{15} \mathrm{H}_{22} \mathrm{~N}_{5} \mathrm{O}(\mathrm{M}+\mathrm{H})^{+}$requires $\mathrm{m} / \mathrm{z} 288.1819$, found $\mathrm{m} / \mathrm{z} 288.1818$.

(1R,2S)-2-(6-Morpholino-9H-purin-9-yl)cyclopentan-1-ol (2i)

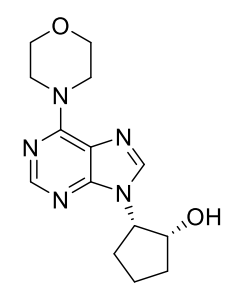

White solid, m.p. $71.2-73.6{ }^{\circ} \mathrm{C} .27 .5 \mathrm{mg}, 95 \%$ yield, $94 \%$ ee; $[\alpha]_{\mathrm{D}}{ }^{20}=-47.22\left(\mathrm{c}=0.360, \mathrm{CH}_{2} \mathrm{Cl}_{2}\right)$; HPLC CHIRALCEL IA, $n$-hexane $/ 2$-propanol $=80 / 20$, flow rate $=0.7 \mathrm{~mL} / \mathrm{min}, \lambda=250 \mathrm{~nm}$, retention time: $13.347 \mathrm{~min}, 16.074 \mathrm{~min}$; TLC: $\mathrm{R}_{\mathrm{f}}=0.31$ (dichloromethane:methanol = 30:1) [UV]; ${ }^{1}$ H NMR $\left(400 \mathrm{MHz}, \mathrm{CDCl}_{3}\right) \delta 8.17(\mathrm{~s}, 1 \mathrm{H}), 7.85(\mathrm{~s}, 1 \mathrm{H}), 4.69-4.63(\mathrm{~m}, 1 \mathrm{H}), 4.50-4.48(\mathrm{~m}, 1 \mathrm{H})$, $4.16(\mathrm{~s}, 4 \mathrm{H}), 3.76(\mathrm{t}, J=4.8 \mathrm{~Hz}, 4 \mathrm{H}), 2.34-2.24(\mathrm{~m}, 1 \mathrm{H}), 2.18-2.00(\mathrm{~m}, 3 \mathrm{H}), 1.91-1.84(\mathrm{~m}, 1 \mathrm{H})$, 1.79-1.67 (m, 1H); ${ }^{13} \mathbf{C}$ NMR (100 MHz, $\left.\mathrm{CDCl}_{3}\right) \delta$ 153.6, 151.9, 150.9, 138.6, 119.7, 71.9, 67.0, 59.5, 45.6, 32.6, 27.8, 20.2; HRMS (ESI-TOF): exact mass calcd for $\mathrm{C}_{14} \mathrm{H}_{20} \mathrm{~N}_{5} \mathrm{O}_{2}(\mathrm{M}+\mathrm{H})^{+}$ requires $\mathrm{m} / \mathrm{z}$ 290.1612, found $\mathrm{m} / \mathrm{z} 290.1615$.

(1R,2S)-2-(6-Bromo-9H-purin-9-yl)cyclopentan-1-ol (2j)

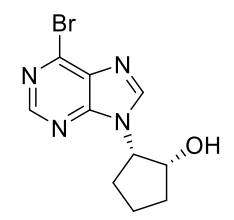

White solid, m.p. $172.2-174.7{ }^{\circ} \mathrm{C} .26 .2 \mathrm{mg}, 93 \%$ yield, $75 \%$ ee; $[\alpha]_{\mathrm{D}}{ }^{20}=-21.62(\mathrm{c}=0.266$, 
$\mathrm{CH}_{2} \mathrm{Cl}_{2}$ ); HPLC CHIRALCEL OD-H, $n$-hexane $/ 2$-propanol $=90 / 10$, flow rate $=0.8 \mathrm{~mL} / \mathrm{min}, \lambda=$ $256 \mathrm{~nm}$, retention time: $17.867 \mathrm{~min}, 24.863 \mathrm{~min}$; TLC: $\mathrm{R}_{\mathrm{f}}=0.36$ (dichloromethane:methanol $=$ 30:1) [UV]; ${ }^{1} \mathrm{H}$ NMR (600 MHz, CD $\left.{ }_{3} \mathrm{OD}\right) \delta$ 8.67-8.66 (m, 2H), 4.98-4.94 (m, 1H), 4.38-4.36 (m, 1H), 2.45-2.38 (m, 1H), 2.31-2.26 (m, 1H), 2.16-2.06 (m, 2H), 1.90-1.78 (m, 2H); ${ }^{13}$ C NMR (150 $\left.\mathrm{MHz}, \mathrm{CDCl}_{3}\right) \delta 151.8,150.6,145.4,142.2,133.4,71.8,59.6,32.8,28.1,20.3$; HRMS (ESI-TOF): exact mass calcd for $\mathrm{C}_{10} \mathrm{H}_{12} \mathrm{BrN}_{4} \mathrm{O}(\mathrm{M}+\mathrm{H})^{+}$requires m/z 283.0189, found m/z 283.0190.

(1R,2S)-2-(6-Chloro-9H-purin-9-yl)cyclopentan-1-ol (2k)

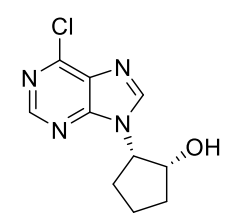

White solid, m.p. $150.9-152.8{ }^{\circ} \mathrm{C} .21 .9 \mathrm{mg}, 92 \%$ yield, $70 \%$ ee; $[\alpha]_{\mathrm{D}}{ }^{20}=-59.64(\mathrm{c}=0.204$, $\mathrm{CH}_{2} \mathrm{Cl}_{2}$ ); HPLC CHIRALCEL ID, $n$-hexane/2-propanol $=90 / 10$, flow rate $=0.6 \mathrm{~mL} / \mathrm{min}, \lambda=256$ $\mathrm{nm}$, retention time: $34.443 \mathrm{~min}, 43.273 \mathrm{~min}$; $\mathbf{T L C}: \mathrm{R}_{\mathrm{f}}=0.35$ (dichloromethane:methanol = 30:1) [UV]; ${ }^{1} \mathbf{H}$ NMR $\left(600 \mathrm{MHz}, \mathrm{CD}_{3} \mathrm{OD}\right) \delta 8.72(\mathrm{~s}, 1 \mathrm{H}), 8.66(\mathrm{~s}, 1 \mathrm{H}), 4.99-4.95(\mathrm{~m}, 1 \mathrm{H}), 4.38-4.36(\mathrm{~m}$, 1H), 2.45-2.38 (m, 1H), 2.31-2.26 (m, 1H), 2.16-2.06 (m, 2H), 1.90-1.78 (m, 2H); ${ }^{13}$ C NMR (150 $\left.\mathrm{MHz}, \mathrm{CDCl}_{3}\right) \delta 151.8,151.6,149.8,145.5,130.4,71.5,59.6,32.7,28.1,20.3 ;$ HRMS (ESI-TOF): exact mass calcd for $\mathrm{C}_{10} \mathrm{H}_{12} \mathrm{ClN} \mathrm{N}_{4} \mathrm{O}(\mathrm{M}+\mathrm{H})^{+}$requires m/z 239.0694, found $\mathrm{m} / \mathrm{z} 239.0697$.

(1R,2S)-2-(2-Chloro-6-(dimethylamino)-9H-purin-9-yl)cyclopentan-1-ol (2l)

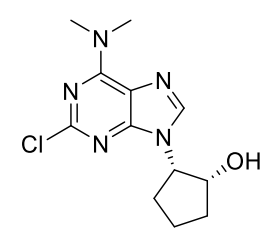

White solid, m.p. $194.3-196.1{ }^{\circ} \mathrm{C} .27 .3 \mathrm{mg}, 97 \%$ yield, $>99 \%$ ee; $[\alpha]_{\mathrm{D}}{ }^{20}=-61.11(\mathrm{c}=0.252$, $\mathrm{CH}_{2} \mathrm{Cl}_{2}$ ); HPLC CHIRALCEL OD-H, $n$-hexane $/ 2$-propanol $=80 / 20$, flow rate $=0.7 \mathrm{~mL} / \mathrm{min}, \lambda=$ $250 \mathrm{~nm}$, retention time: $9.600 \mathrm{~min}$; TLC: $\mathrm{R}_{\mathrm{f}}=0.36$ (dichloromethane: methanol $=30: 1$ ) [UV]; ${ }^{1} \mathbf{H}$ NMR (600 MHz, $\left.\mathrm{CDCl}_{3}\right) \delta 7.71(\mathrm{~s}, 1 \mathrm{H}), 4.70-4.66(\mathrm{~m}, 1 \mathrm{H}), 4.47$ (t, J=4.8 Hz, 1H), $3.92(\mathrm{~s}, 1 \mathrm{H})$, 3.45-3.21 (m, 6H), 2.29-2.22 (m, 1H), 2.15-2.10 (m, 2H), 2.04-2.01 (m, 1H), 1.90-1.85 (m, $1 \mathrm{H})$, 1.75-1.68 (m, 1H); ${ }^{13} \mathbf{C}$ NMR (100 MHz, $\left.\mathrm{CDCl}_{3}\right) \delta$ 154.3, 153.5, 151.4, 138.6, 118.1, 71.6, 59.1, 38.4, 32.2, 27.2, 20.1; HRMS (ESI-TOF): exact mass calcd for $\mathrm{C}_{12} \mathrm{H}_{17} \mathrm{ClN}_{5} \mathrm{O}(\mathrm{M}+\mathrm{H})^{+}$requires $\mathrm{m} / \mathrm{z} 282.1116$, found $\mathrm{m} / \mathrm{z} 282.1118$.

(1R,2S)-2-(2-Chloro-6-(diethylamino)-9H-purin-9-yl)cyclopentan-1-ol (2m) 


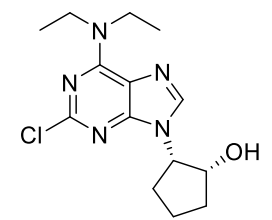

White solid, m.p. $110.7-113.2{ }^{\circ} \mathrm{C} .28 .7 \mathrm{mg}, 93 \%$ yield, $95 \%$ ee; $[\alpha]_{\mathrm{D}}{ }^{20}=-53.20(\mathrm{c}=0.500$, $\mathrm{CH}_{2} \mathrm{Cl}_{2}$ ); HPLC CHIRALCEL IE, $n$-hexane $/ 2$-propanol $=80 / 20$, flow rate $=0.8 \mathrm{~mL} / \mathrm{min}, \lambda=250$ $\mathrm{nm}$, retention time: $8.052 \mathrm{~min}, 10.518 \mathrm{~min}$; TLC: $\mathrm{R}_{\mathrm{f}}=0.31$ (dichloromethane: methanol $=30: 1$ ) [UV]; ${ }^{1} \mathbf{H}$ NMR $\left(400 \mathrm{MHz}, \mathrm{CDCl}_{3}\right) \delta 7.85(\mathrm{~s}, 1 \mathrm{H}), 4.74-4.68(\mathrm{~m}, 1 \mathrm{H}), 4.46-4.43(\mathrm{~m}, 1 \mathrm{H})$, 3.97-3.72 (m, 4H), $2.85(\mathrm{~s}, 1 \mathrm{H}), 2.31-1.99(\mathrm{~m}, 4 \mathrm{H}), 1.90-1.82(\mathrm{~m}, 1 \mathrm{H}), 1.79-1.67(\mathrm{~m}, 1 \mathrm{H}), 1.25(\mathrm{t}$, $J=7.2 \mathrm{~Hz}, 6 \mathrm{H}) .{ }^{13} \mathbf{C}$ NMR $\left(100 \mathrm{MHz}, \mathrm{CDCl}_{3}\right) \delta 153.9,153.7,151.9,138.8,118.2,72.1,58.9$, 43.2, 32.6, 27.8, 20.2, 14.2, 12.9; HRMS (ESI-TOF): exact mass calcd for $\mathrm{C}_{14} \mathrm{H}_{21} \mathrm{ClN}_{5} \mathrm{O}(\mathrm{M}+\mathrm{H})^{+}$ requires $\mathrm{m} / \mathrm{z} 310.1429$, found $\mathrm{m} / \mathrm{z} 310.1428$.

(1R,2S)-2-(2-Chloro-6-(pyrrolidin-1-yl)-9H-purin-9-yl)cyclopentan-1-ol (2n)

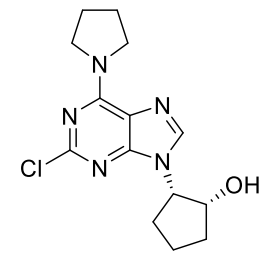

White solid, m.p. $193.1-196.4{ }^{\circ} \mathrm{C} .28 .6 \mathrm{mg}, 93 \%$ yield, $99 \%$ ee; $[\alpha]_{\mathrm{D}^{20}}=-40.83(\mathrm{c}=0.516$, $\mathrm{CH}_{2} \mathrm{Cl}_{2}$ ); HPLC CHIRALCEL ID, $n$-hexane $/ 2$-propanol $=70 / 30$, flow rate $=0.7 \mathrm{~mL} / \mathrm{min}, \lambda=250$ $\mathrm{nm}$, retention time: $11.026 \mathrm{~min}, 12.553 \mathrm{~min}$; TLC: $\mathrm{R}_{\mathrm{f}}=0.27$ (dichloromethane:methanol $=30: 1$ ) [UV]; ${ }^{1} \mathbf{H}$ NMR $\left(600 \mathrm{MHz}, \mathrm{CDCl}_{3}\right) \delta 7.68(\mathrm{~s}, 1 \mathrm{H}), 4.68-4.64(\mathrm{~m}, 1 \mathrm{H}), 4.49-4.47(\mathrm{~m}, 1 \mathrm{H})$, 4.30-4.298 (m, 1H), 3.92-3.88 (m, 1H), 3.64-3.57 (m, 3H), 2.29-2.22 (m, 1H), 2.16-2.01 (m, 5H), 1.97-1.85 (m, 3H), 1.74-1.66 (m, 1H); ${ }^{13} \mathbf{C}$ NMR (100 MHz, $\left.\mathrm{CDCl}_{3}\right) \delta 153.9,152.6,151.0,139.2$, 118.3, 71.7, 59.2, 48.5, 47.8, 32.4, 27.5, 26.2, 24.0, 20.3; HRMS (ESI-TOF): exact mass calcd for $\mathrm{C}_{14} \mathrm{H}_{19} \mathrm{ClN}_{5} \mathrm{O}(\mathrm{M}+\mathrm{H})^{+}$requires $\mathrm{m} / \mathrm{z} 308.1273$, found $\mathrm{m} / \mathrm{z} 308.1270$.

(1R,2S)-2-(2-Chloro-6-morpholino-9H-purin-9-yl)cyclopentan-1-ol (2o)

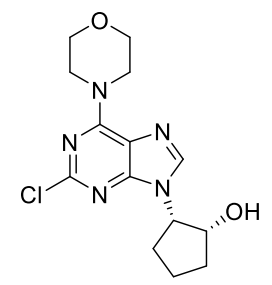

White solid, m.p. $211.7-215.2^{\circ} \mathrm{C}, 31.3 \mathrm{mg}, 97 \%$ yield, $94 \%$ ee; $[\alpha]_{\mathrm{D}}{ }^{20}=-59.39\left(\mathrm{c}=0.284, \mathrm{CH}_{2} \mathrm{Cl}_{2}\right)$; HPLC CHIRALCEL OD-H, $n$-hexane $/ 2$-propanol $=90 / 10$, flow rate $=0.8 \mathrm{~mL} / \mathrm{min}, \lambda=256 \mathrm{~nm}$, 
retention time: $15.060 \mathrm{~min}, 19.737 \mathrm{~min}$; TLC: $\mathrm{R}_{\mathrm{f}}=0.32$ (dichloromethane:methanol = 30:1) [UV]; ${ }^{1}$ H NMR $\left(600 \mathrm{MHz}, \mathrm{CDCl}_{3}\right) \delta 7.91(\mathrm{~s}, 1 \mathrm{H}), 4.77-4.73(\mathrm{~m}, 1 \mathrm{H}), 4.46-4.45(\mathrm{~m}, 1 \mathrm{H}), 4.27(\mathrm{~s}, 4 \mathrm{H})$, 3.81-3.80 (m, 4H), 2.28-2.18 (m, 3H), 2.13-2.01 (m, 2H), 1.89-1.84 (m, 1H), 1.78-1.72 (m, 1H); ${ }^{13} \mathrm{C}$ NMR $\left(100 \mathrm{MHz}, \mathrm{CDCl}_{3}\right) \delta 153.8,153.7,152.4,139.0,118.5,72.0,67.0,58.8,45.4,32.8$, 28.0, 20.2; HRMS (ESI-TOF): exact mass calcd for $\mathrm{C}_{14} \mathrm{H}_{19} \mathrm{ClN}_{5} \mathrm{O}_{2}(\mathrm{M}+\mathrm{H})^{+}$requires $\mathrm{m} / \mathrm{z} 324.1222$, found $\mathrm{m} / \mathrm{z} 324.1222$.

(1R,2S)-2-(2-Amino-6-chloro-9H-purin-9-yl)cyclopentan-1-ol (2p)

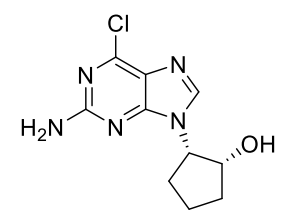

White solid, m.p. $174.2-178.8{ }^{\circ} \mathrm{C} .21 .8 \mathrm{mg}, 86 \%$ yield, $90 \%$ ee; $[\alpha]_{\mathrm{D}^{20}}=-73.13(\mathrm{c}=0.098$, $\mathrm{CH}_{2} \mathrm{Cl}_{2}$ ); HPLC CHIRALCEL ID, $n$-hexane $/ 2$-propanol $=80 / 20$, flow rate $=0.7 \mathrm{~mL} / \mathrm{min}, \lambda=250$ $\mathrm{nm}$, retention time: $17.493 \mathrm{~min}, 22.997 \mathrm{~min}$; TLC: $\mathrm{R}_{\mathrm{f}}=0.27$ (dichloromethane:methanol $=30: 1$ ) [UV]; ${ }^{1} \mathbf{H}$ NMR (400 MHz, CD $\left.{ }_{3} \mathrm{OD}\right) \delta 8.18(\mathrm{~s}, 1 \mathrm{H}), 4.73-4.67(\mathrm{~m}, 1 \mathrm{H}), 4.33-4.31(\mathrm{~m}, 1 \mathrm{H})$, 2.35-2.25 (m, 1H), 2.22-2.15 (m, 1H), 2.10-2.00 (m, 2H), 1.88-1.72 (m, 2H); ${ }^{13} \mathbf{C}$ NMR (100 MHz, $\left.\mathrm{CD}_{3} \mathrm{OD}\right) \delta 161.4,155.7,151.2,144.2,124.8,72.3,60.0,33.5,28.4,21.0$; HRMS (ESI-TOF): exact mass calcd for $\mathrm{C}_{10} \mathrm{H}_{13} \mathrm{ClN}_{5} \mathrm{O}(\mathrm{M}+\mathrm{H})^{+}$requires $\mathrm{m} / \mathrm{z} 254.0803$, found $\mathrm{m} / \mathrm{z} 254.0808$.

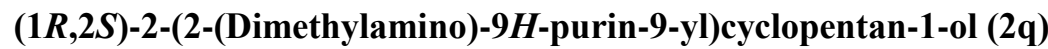

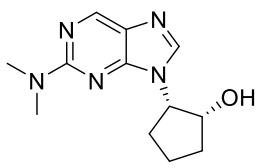

White solid, m.p. $126.2-128.4{ }^{\circ} \mathrm{C} .22 .2 \mathrm{mg}, 90 \%$ yield, $91 \%$ ee; $[\alpha]_{\mathrm{D}}^{20}=-49.28(\mathrm{c}=0.346$, $\mathrm{CH}_{2} \mathrm{Cl}_{2}$ ); HPLC CHIRALCEL OD-H, $n$-hexane $/ 2$-propanol $=90 / 10$, flow rate $=0.8 \mathrm{~mL} / \mathrm{min}, \lambda=$ $256 \mathrm{~nm}$, retention time: $11.570 \mathrm{~min}, 18.253 \mathrm{~min}$; TLC: $\mathrm{R}_{\mathrm{f}}=0.32$ (dichloromethane:methanol $=$ 30:1) [UV]; ${ }^{1} \mathrm{H}$ NMR (400 MHz, $\left.\mathrm{CDCl}_{3}\right) \delta 8.46(\mathrm{~s}, 1 \mathrm{H}), 7.81(\mathrm{~s}, 1 \mathrm{H}), 4.60-4.54(\mathrm{~m}, 2 \mathrm{H}), 3.18(\mathrm{~s}$, $6 \mathrm{H}), 2.32-2.22(\mathrm{~m}, 1 \mathrm{H}), 2.16-2.03(\mathrm{~m}, 3 \mathrm{H}), 1.97-1.91(\mathrm{~m}, 1 \mathrm{H}), 1.79-1.69(\mathrm{~m}, 1 \mathrm{H}) ;{ }^{13} \mathbf{C}$ NMR $(100$ $\left.\mathrm{MHz}, \mathrm{CDCl}_{3}\right) \delta 159.5,152.9,148.7,142.0,125.5,71.8,59.6,37.8,32.5,27.8,20.2 ;$ HRMS (ESI-TOF): exact mass calcd for $\mathrm{C}_{12} \mathrm{H}_{17} \mathrm{~N}_{5} \mathrm{NaO}(\mathrm{M}+\mathrm{Na})^{+}$requires $\mathrm{m} / \mathrm{z} 270.1325$, found $\mathrm{m} / \mathrm{z}$ 270.1331.

(1R,2S)-2-(6-(Dimethylamino)-9H-purin-9-yl)cyclohexan-1-ol (2r) 


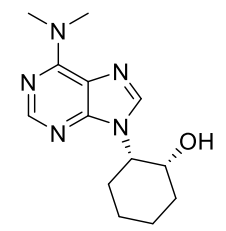

White solid, m.p. 137.2-139.0 ${ }^{\circ} \mathrm{C} .24 .0 \mathrm{mg}, 92 \%$ yield, $99 \%$ ee; $[\alpha]_{\mathrm{D}}{ }^{20}=-20.41(\mathrm{c}=0.392$,

$\mathrm{CH}_{2} \mathrm{Cl}_{2}$ ); HPLC CHIRALCEL IE, $n$-hexane/2-propanol $=80 / 20$, flow rate $=0.7 \mathrm{~mL} / \mathrm{min}, \lambda=250$ nm, retention time: $25.714 \mathrm{~min}, 29.236 \mathrm{~min}$; $\mathbf{T L C}: \mathrm{R}_{\mathrm{f}}=0.43$ (dichloromethane: methanol = 30:1) [UV]; ${ }^{1} \mathbf{H}$ NMR $\left(600 \mathrm{MHz}, \mathrm{CDCl}_{3}\right) \delta 8.21(\mathrm{~s}, 1 \mathrm{H}), 7.80(\mathrm{~s}, 1 \mathrm{H}), 4.45-4.42(\mathrm{~m}, 1 \mathrm{H}), 4.31-4.30(\mathrm{~m}$, 1H), $3.42(\mathrm{~s}, 6 \mathrm{H}), 2.41-2.34(\mathrm{~m}, 1 \mathrm{H}), 1.99-1.91(\mathrm{~m}, 2 \mathrm{H}), 1.82-1.66(\mathrm{~m}, 3 \mathrm{H}), 1.55-1.44(\mathrm{~m}, 2 \mathrm{H})$; ${ }^{13} \mathbf{C}$ NMR $\left(150 \mathrm{MHz} \mathrm{CDCl}_{3}\right) \delta 154.7,151.7,149.8,138.2,120.0,67.6,58.7,38.6,32.5,26.2$, 25.7, 18.9; HRMS (ESI-TOF): exact mass calcd for $\mathrm{C}_{13} \mathrm{H}_{20} \mathrm{~N}_{5} \mathrm{O}(\mathrm{M}+\mathrm{H})^{+}$requires $\mathrm{m} / \mathrm{z} 262.1662$, found $\mathrm{m} / \mathrm{z} 262.1663$.

(1R,2S)-2-(6-(Dimethylamino)-9H-purin-9-yl)cycloheptan-1-ol (2s)

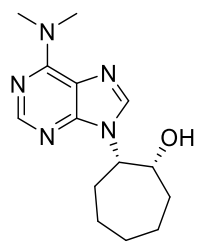

White solid, m.p. $137.8-140.5{ }^{\circ} \mathrm{C} .21 .5 \mathrm{mg}, 78 \%$ yield, $99 \%$ ee; $[\alpha]_{\mathrm{D}}{ }^{20}=-13.36(\mathrm{c}=0.378$, $\mathrm{CH}_{2} \mathrm{Cl}_{2}$ ); HPLC CHIRALCEL IE, $n$-hexane/2-propanol $=80 / 20$, flow rate $=0.8 \mathrm{~mL} / \mathrm{min}, \lambda=250$ $\mathrm{nm}$, retention time: $23.859 \mathrm{~min}, 27.243 \mathrm{~min}$;LC: $\mathrm{R}_{\mathrm{f}}=0.51$ (dichloromethane: methanol = 30:1) [UV]; ${ }^{1} \mathbf{H}$ NMR $\left(400 \mathrm{MHz}, \mathrm{CDCl}_{3}\right) \delta 8.24(\mathrm{~s}, 1 \mathrm{H}), 7.73(\mathrm{~s}, 1 \mathrm{H}), 4.48-4.45(\mathrm{~m}, 1 \mathrm{H}), 4.33(\mathrm{t}, J=4.4$ $\mathrm{Hz}, 1 \mathrm{H}), 3.49(\mathrm{~s}, 6 \mathrm{H}), 2.59-2.48(\mathrm{~m}, 1 \mathrm{H}), 2.04-1.96(\mathrm{~m}, 1 \mathrm{H}), 1.88-1.53(\mathrm{~m}, 8 \mathrm{H}) ;{ }^{13} \mathbf{C} \mathbf{N M R}(100$ $\left.\mathrm{MHz}_{\mathrm{CDCl}}\right) \delta 155.0,151.5,149.5,138.4,120.5,72.0,62.3,38.7,33.8,29.0,26.5,25.4,23.4$; HRMS (ESI-TOF): exact mass calcd for $\mathrm{C}_{14} \mathrm{H}_{21} \mathrm{~N}_{5} \mathrm{NaO}(\mathrm{M}+\mathrm{Na})^{+}$requires $\mathrm{m} / \mathrm{z}$ 298.1638, found $\mathrm{m} / \mathrm{z} 298.1642$.

$(1 R, 2 S)-2-(6-(D i m e t h y l a m i n o)-9 H-p u r i n-9-y l)-2,3-d i h y d r o-1 H$-inden-1-ol (2t)

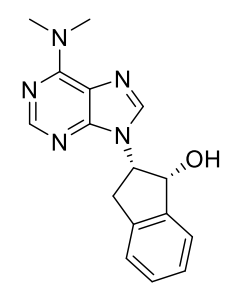

White solid, m.p. $242.3-253.2{ }^{\circ} \mathrm{C} .26 .3 \mathrm{mg}, 89 \%$ yield, $98 \%$ ee; $[\alpha]_{\mathrm{D}}{ }^{20}=3.56\left(\mathrm{c}=0.440, \mathrm{CH}_{2} \mathrm{Cl}_{2}\right)$; 
HPLC CHIRALCEL OD-H, $n$-hexane $/ 2$-propanol $=90 / 10$, flow rate $=0.8 \mathrm{~mL} / \mathrm{min}, \lambda=256 \mathrm{~nm}$, retention time: $23.597 \mathrm{~min}, 27.037 \mathrm{~min}$; TLC: $\mathrm{R}_{\mathrm{f}}=0.36$ (dichloromethane:methanol = 30:1) [UV]; ${ }^{1} \mathbf{H}$ NMR $\left(600 \mathrm{MHz}, \mathrm{CDCl}_{3}\right) \delta 8.26(\mathrm{~s}, 1 \mathrm{H}), 7.72(\mathrm{~s}, 1 \mathrm{H}), 7.51-7.50(\mathrm{~m}, 1 \mathrm{H}), 7.38-7.32(\mathrm{~m}, 3 \mathrm{H})$, $5.43(\mathrm{~d}, J=5.4 \mathrm{~Hz}, 1 \mathrm{H}), 5.34-5.30(\mathrm{~m}, 1 \mathrm{H}), 3.66-3.41(\mathrm{~m}, 8 \mathrm{H}) ;{ }^{13} \mathbf{C}$ NMR $\left(150 \mathrm{MHz}, \mathrm{CDCl}_{3}\right) \delta$ $154.9,152.1,150.8,142.0,139.5,138.2,129.5,128.0,125.7,125.0,119.9,75.3,58.3,35.9$; HRMS (ESI-TOF): exact mass calcd for $\mathrm{C}_{16} \mathrm{H}_{17} \mathrm{~N}_{5} \mathrm{NaO}(\mathrm{M}+\mathrm{Na})^{+}$requires $\mathrm{m} / \mathrm{z} 318.1325$, found $\mathrm{m} / \mathrm{z} 318.1333$.

$(1 R, 2 S)-2-(1 H-B e n z o[d]$ imidazol-1-yl)cyclopentan-1-ol (4a)

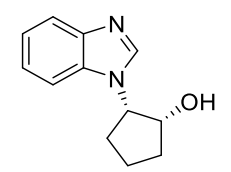

White oil, $6.5 \mathrm{mg}, 32 \%$ yield, $>20 / 1 \mathrm{dr}$, 9\% ee.; $[\alpha]_{\mathrm{D}}{ }^{20}=2.65\left(\mathrm{c}=0.365, \mathrm{CH}_{2} \mathrm{Cl}_{2}\right)$; HPLC CHIRALCEL OD-H, $n$-hexane/2-propanol $=90 / 10$, flow rate $=0.6 \mathrm{~mL} / \mathrm{min}, \lambda=256 \mathrm{~nm}$, retention time: $19.383 \mathrm{~min}, 24.047 \mathrm{~min}$; TLC: $\mathrm{R}_{\mathrm{f}}=0.36$ (dichloromethane:methanol $=30: 1$ ) [UV]; ${ }^{1} \mathbf{H}$ NMR $\left(400 \mathrm{MHz}, \mathrm{CDCl}_{3}\right) \delta 7.98(\mathrm{~s}, 1 \mathrm{H}), 7.43(\mathrm{t}, J=8.0 \mathrm{~Hz}, 1 \mathrm{H}), 7.33(\mathrm{~d}, J=8.0 \mathrm{~Hz}, 1 \mathrm{H}), 7.18(\mathrm{~d}$, $J=6.4 \mathrm{~Hz}, 1 \mathrm{H}), 7.10-7.05(\mathrm{~m}, 1 \mathrm{H}), 4.52-4.46(\mathrm{~m}, 2 \mathrm{H}), 2.48-2.37(\mathrm{~m}, 1 \mathrm{H}), 2.20-2.08(\mathrm{~m}, 3 \mathrm{H})$, 2.01-1.94 (m, 1H), 1.83-1.76 (m, 1H); ${ }^{13} \mathbf{C}$ NMR (150 MHz, $\left.\mathrm{CDCl}_{3}\right) \delta 143.0,142.1,134.0,122.8$, 122.3, 119.9, 109.7, 71.5, 60.7, 32.2, 27.2, 20.1; HRMS (ESI-TOF): exact mass calcd for $\mathrm{C}_{12} \mathrm{H}_{15} \mathrm{~N}_{2} \mathrm{O}(\mathrm{M}+\mathrm{H})^{+}$requires $\mathrm{m} / \mathrm{z} 203.1179$, found $\mathrm{m} / \mathrm{z} 203.1179$.

(1R,2S)-2-(1H-Benzo[d][1,2,3]triazol-1-yl)cyclopentan-1-ol (4b)

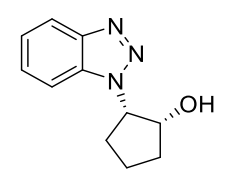

White solid, m.p. $81.8-82.9{ }^{\circ} \mathrm{C} .14 .8 \mathrm{mg}, 73 \%$ yield, $>20 / 1 \mathrm{dr}, 25 \%$ ee; $[\alpha]_{\mathrm{D}}{ }^{20}=-0.75(\mathrm{c}=0.530$, $\mathrm{CH}_{2} \mathrm{Cl}_{2}$ ); HPLC CHIRALCEL ID, $n$-hexane/2-propanol $=90 / 10$, flow rate $=0.8 \mathrm{~mL} / \mathrm{min}, \lambda=256$ $\mathrm{nm}$, retention time: $41.810 \mathrm{~min}, 61.957 \mathrm{~min}$; TLC: $\mathrm{R}_{\mathrm{f}}=0.41$ (dichloromethane:methanol $=30: 1$ ) [UV]; ${ }^{1} \mathbf{H}$ NMR $\left(600 \mathrm{MHz}, \mathrm{CDCl}_{3}\right) \delta 8.02(\mathrm{~d}, J=7.8 \mathrm{~Hz}, 1 \mathrm{H}), 7.60(\mathrm{~d}, J=8.4 \mathrm{~Hz}, 1 \mathrm{H}), 7.49$ (t, $J$ $=7.8 \mathrm{~Hz}, 1 \mathrm{H}), 7.38(\mathrm{t}, J=7.8 \mathrm{~Hz}, 1 \mathrm{H}), 4.94-4.90(\mathrm{~m}, 1 \mathrm{H}), 4.74-4.71(\mathrm{~m}, 1 \mathrm{H}), 3.06(\mathrm{~s}, 1 \mathrm{H})$, 2.57-2.50 (m, 1H), 2.41-2.36 (m, 1H), 2.25-2.18 (m, 1H), 2.12-2.02 (m, 2H), 1.89-1.82 (m, 1H); ${ }^{13}$ C NMR (150 MHz, $\left.\mathrm{CDCl}_{3}\right) \delta 145.8,133.8,127.5,124.3,120.1,110.3,74.1,63.4,32.1,27.9$, 
20.6; HRMS (ESI-TOF): exact mass calcd for $\mathrm{C}_{11} \mathrm{H}_{13} \mathrm{~N}_{3} \mathrm{NaO}(\mathrm{M}+\mathrm{Na})^{+}$requires $\mathrm{m} / \mathrm{z} 226.0951$, found $\mathrm{m} / \mathrm{z} 226.0958$.

$(1 R, 2 S)$-2-(1H-Imidazo[4,5-c]pyridin-1-yl)cyclopentan-1-ol (4c)

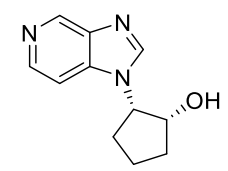

White oil, $18.5 \mathrm{mg}, 91 \%$ yield, $>20 / 1 \mathrm{dr}, 38 \%$ ee; $[\alpha]_{\mathrm{D}}{ }^{20}=22.73\left(\mathrm{c}=0.404, \mathrm{CH}_{2} \mathrm{Cl}_{2}\right)$; TLC: $\mathrm{R}_{\mathrm{f}}=$ 0.23 (dichloromethane:methanol = 30:1) [UV]; ${ }^{1} \mathbf{H}$ NMR $\left(400 \mathrm{MHz}, \mathrm{CDCl}_{3}\right) \delta 8.51(\mathrm{~s}, 1 \mathrm{H})$, 8.08-8.07 (m, 1H), 7.94-7.91 (m, 1H), 7.08-7.04 (m, 1H), 4.53-4.47 (m, 2H), 2.48-2.38 (m, 1H), 2.23-2.09 (m, 3H), 2.04-1.96 (m, 1H), 1.87-1.75 (m, 1H); ${ }^{13} \mathbf{C}$ NMR (100 MHz, $\left.\mathrm{CDCl}_{3}\right) \delta 147.8$, 145.0, 141.2, 133.0, 131.7, 114.3, 71.1, 61.3, 32.4, 27.3, 20.2; HRMS (ESI-TOF): exact mass calcd for $\mathrm{C}_{11} \mathrm{H}_{14} \mathrm{~N}_{3} \mathrm{O}(\mathrm{M}+\mathrm{H})^{+}$requires $\mathrm{m} / \mathrm{z}$ 204.1131, found $\mathrm{m} / \mathrm{z} 204.1138$.

1-((1S,2R)-2-((Trimethylsilyl)oxy)cyclopentyl)-1 $H$-imidazo[4,5-c]pyridine (4c')

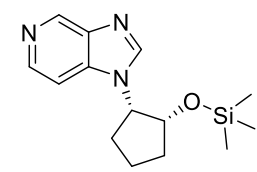

White oil, 93\% yield, >20/1 dr, 38\% ee; HPLC CHIRALCEL ID, $n$-hexane/2-propanol = 80/20, flow rate $=0.7 \mathrm{~mL} / \mathrm{min}, \lambda=250 \mathrm{~nm}$, retention time: $15.689 \mathrm{~min}, 22.691 \mathrm{~min}$; TLC: $\mathrm{R}_{\mathrm{f}}=0.34$ (dichloromethane:methanol $=30: 1)[\mathrm{UV}] ;{ }^{1} \mathbf{H}$ NMR $\left(400 \mathrm{MHz}, \mathrm{CDCl}_{3}\right) \delta 8.85(\mathrm{~s}, 1 \mathrm{H}), 8.43(\mathrm{~d}, J$ $=5.6 \mathrm{~Hz}, 1 \mathrm{H}), 8.19(\mathrm{~s}, 1 \mathrm{H}), 7.69(\mathrm{~d}, J=5.2 \mathrm{~Hz}, 1 \mathrm{H}), 4.66-4.60(\mathrm{~m}, 1 \mathrm{H}), 4.29-2.27(\mathrm{~m}, 1 \mathrm{H})$, 2.48-2.37 (m, 1H), 2.27-2.19 (m, 1H), 2.14-2.01 (m, 2H), 1.88-1.76 (m, 2H), -0.26 (s, 9H); ${ }^{13} \mathrm{C}$ NMR $\left(100 \mathrm{MHz}, \mathrm{CDCl}_{3}\right) \delta 148.7,145.4,141.9,133.7,132.3,115.0,72.6,60.7,33.5,27.9,20.3$, -0.5 ; HRMS (ESI-TOF): exact mass calcd for $\mathrm{C}_{14} \mathrm{H}_{22} \mathrm{~N}_{3} \mathrm{OSi}(\mathrm{M}+\mathrm{H})^{+}$requires $\mathrm{m} / \mathrm{z} 276.1527$, found $\mathrm{m} / \mathrm{z} 276.1529$.

(1R,2S)-2-(7H-Purin-7-yl)cyclopentan-1-ol (4d)

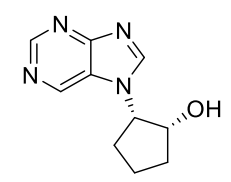

White solid, m.p. $187.9-193.5^{\circ} \mathrm{C}, 8.8 \mathrm{mg}, 43 \%$ yield, $>20 / 1 \mathrm{dr}, 44 \%$ ee; $[\alpha]_{\mathrm{D}}^{20}=-1.75(\mathrm{c}=0.114$, $\mathrm{CH}_{2} \mathrm{Cl}_{2}$ ); HPLC CHIRALCEL ID, $n$-hexane $/ 2$-propanol $=25 / 75$, flow rate $=0.3 \mathrm{~mL} / \mathrm{min}, \lambda=250$ $\mathrm{nm}$, retention time: $17.748 \mathrm{~min}, 26.477 \mathrm{~min}$; TLC: $\mathrm{R}_{\mathrm{f}}=0.26$ (dichloromethane: methanol $=30: 1$ ) 
[UV]; ${ }^{1} \mathrm{H}$ NMR $\left(600 \mathrm{MHz}, \mathrm{CD}_{3} \mathrm{OD}\right) \delta 9.22(\mathrm{~s}, 1 \mathrm{H}), 8.97(\mathrm{~s}, 1 \mathrm{H}), 8.73(\mathrm{~s}, 1 \mathrm{H}), 4.91-4.87(\mathrm{~m}, 1 \mathrm{H})$, 4.40-4.38 (m, 1H), 2.49-2.43 (m, 1H), 2.33-2.28 (m, 1H), 2.16-2.08 (m, 2H), 1.89-1.81 (m, 2H); ${ }^{13} \mathrm{C}$ NMR $\left(150 \mathrm{MHz}, \mathrm{CD}_{3} \mathrm{OD}\right) \delta 161.2,153.2,150.3,142.9,127.6,73.1,62.9,33.4,28.3,21.0$; HRMS (ESI-TOF): exact mass calcd for $\mathrm{C}_{10} \mathrm{H}_{12} \mathrm{~N}_{4} \mathrm{NaO}(\mathrm{M}+\mathrm{Na})^{+}$requires $\mathrm{m} / \mathrm{z}$ 227.0903, found $\mathrm{m} / \mathrm{z} 227.0910$.

$(1 R, 2 S)$-2-(3H-Imidazo[4,5-b]pyridin-3-yl)cyclopentan-1-ol (4e)

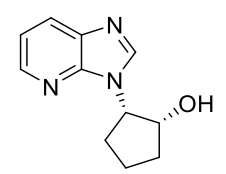

Yellow oil, $18.5 \mathrm{mg}, 91 \%$ yield, $>20 / 1 \mathrm{dr}, 90 \%$ ee. $[\alpha]_{\mathrm{D}^{20}}=-96.08\left(\mathrm{c}=0.332, \mathrm{CH}_{2} \mathrm{Cl}_{2}\right) ; \mathbf{H P L C}$ CHIRALCEL AS-H, $n$-hexane $/ 2$-propanol $=80 / 20$, flow rate $=0.8 \mathrm{~mL} / \mathrm{min}, \lambda=250 \mathrm{~nm}$, retention time: 7.143 min, 11.993 min; TLC: $R_{\mathrm{f}}=0.46$ (dichloromethane:methanol = 30:1) [UV]; ${ }^{1} \mathbf{H}$ NMR $\left(400 \mathrm{MHz}, \mathrm{CD}_{3} \mathrm{OD}\right) \delta 8.53(\mathrm{~s}, 1 \mathrm{H}), 8.39(\mathrm{dd}, J=4.8,1.6 \mathrm{~Hz}, 1 \mathrm{H}), 8.08(\mathrm{dd}, J=8.0,1.2 \mathrm{~Hz}, 1 \mathrm{H})$, $7.34(\mathrm{dd}, J=8.0,4.8 \mathrm{~Hz}, 1 \mathrm{H}), 5.02-4.96(\mathrm{~m}, 1 \mathrm{H}), 4.38-4.35(\mathrm{~m}, 1 \mathrm{H}), 2.44-2.34(\mathrm{~m}, 1 \mathrm{H}), 2.28-2.20$ (m, 1H), 2.18-2.03 (m, 2H), 1.92-1.77 (m, 2H). $\left.{ }^{13} \mathbf{C ~ N M R ~ ( 1 5 0 ~ M H z , ~} \mathrm{CDCl}_{3}\right) \delta 146.4,143.8$, 143.6, 134.2, 126.8, 118.3, 71.5, 59.1, 32.3, 27.6, 20.2; HRMS (ESI-TOF): exact mass calcd for $\mathrm{C}_{11} \mathrm{H}_{14} \mathrm{~N}_{3} \mathrm{O}(\mathrm{M}+\mathrm{H})^{+}$requires $\mathrm{m} / \mathrm{z} 204.1131$, found $\mathrm{m} / \mathrm{z} 204.1132$.

$(1 R, 2 S)-2-(9 H-P u r i n-9-y l) c y c l o p e n t a n-1-o l(4 f)$

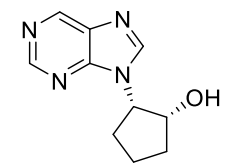

White solid, m.p. $130.4-133.8{ }^{\circ} \mathrm{C}, 18.6 \mathrm{mg}, 91 \%$ yield, $>20 / 1 \mathrm{dr}, 82 \%$ ee; $[\alpha]_{\mathrm{D}}^{20}=-72.97(\mathrm{c}=$ 0.074, $\left.\mathrm{CH}_{2} \mathrm{Cl}_{2}\right) ;$ HPLC CHIRALCEL AS-H, $n$-hexane/2-propanol $=80 / 20$, flow rate $=0.8$ $\mathrm{mL} / \mathrm{min}, \quad \lambda=250 \mathrm{~nm}$, retention time: $9.694 \mathrm{~min}, 13.461 \mathrm{~min} ; \quad$ TLC: $\mathrm{R}_{\mathrm{f}}=0.31$ (dichloromethane:methanol = 30:1) [UV]; ${ }^{1} \mathbf{H}$ NMR $\left(600 \mathrm{MHz}, \mathrm{CD}_{3} \mathrm{OD}\right) \delta 9.07(\mathrm{~s}, 1 \mathrm{H}), 8.93(\mathrm{~s}$, $1 \mathrm{H}), 8.65(\mathrm{~s}, 1 \mathrm{H}), 5.02-4.98(\mathrm{~m}, 1 \mathrm{H}), 4.38(\mathrm{~s}, 1 \mathrm{H}), 2.46-2.38(\mathrm{~m}, 1 \mathrm{H}), 2.31-2.25(\mathrm{~m}, 1 \mathrm{H})$, 2.16-2.08 (m, 2H), 1.91-1.80 (m, 2H); ${ }^{13} \mathbf{C}$ NMR (150 MHz, CD $\left.{ }_{3} \mathrm{OD}\right) \delta 152.2,151.4,147.8,145.5$, 133.5, 71.7, 59.1, 32.8, 28.0, 20.3; HRMS (ESI-TOF): exact mass calcd for $\mathrm{C}_{10} \mathrm{H}_{13} \mathrm{~N}_{4} \mathrm{O}(\mathrm{M}+\mathrm{H})^{+}$ requires $\mathrm{m} / \mathrm{z} 205.1084$, found $\mathrm{m} / \mathrm{z} 205.1084$.

9-((1S,2R)-2-Hydroxycyclopentyl)-1,3-dimethyl-3,9-dihydro-1H-purine-2,6-dione (4g) 


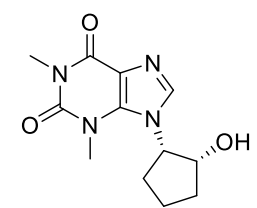

White solid, m.p. $138.2-139.8{ }^{\circ} \mathrm{C} .23 .8 \mathrm{mg}, 90 \%$ yield, $91 \%$ ee; $[\alpha]_{\mathrm{D}}{ }^{20}=-141.37(\mathrm{c}=0.394$, $\mathrm{CH}_{2} \mathrm{Cl}_{2}$ ); HPLC CHIRALCEL IA, $n$-hexane/2-propanol $=70 / 30$, flow rate $=0.6 \mathrm{~mL} / \mathrm{min}, \lambda=256$ nm, retention time: $21.967 \mathrm{~min}, 29.520 \mathrm{~min}$; TLC: $\mathrm{R}_{\mathrm{f}}=0.35$ (dichloromethane:methanol = 30:1) [UV]; ${ }^{1} \mathbf{H}$ NMR $\left(600 \mathrm{MHz}, \mathrm{CDCl}_{3}\right) \delta 7.74(\mathrm{~s}, 1 \mathrm{H}), 5.03(\mathrm{~s}, 1 \mathrm{H}), 4.43(\mathrm{~s}, 1 \mathrm{H}), 3.48-3.29(\mathrm{~m}, 6 \mathrm{H})$, 2.24-2.02(m, 4H) , 1.85-1.73 (m, 2H); ${ }^{13} \mathbf{C}$ NMR $\left(150 \mathrm{MHz}, \mathrm{CDCl}_{3}\right) \delta 155.7,151.3,148.6,140.8$, 107.3, 72.3, 61.8, 32.3, 29.8, 28.2, 27.6, 20.1; HRMS (ESI-TOF): exact mass calcd for $\mathrm{C}_{12} \mathrm{H}_{17} \mathrm{~N}_{4} \mathrm{O}_{3}(\mathrm{M}+\mathrm{H})^{+}$requires $\mathrm{m} / \mathrm{z} 265.1295$, found $\mathrm{m} / \mathrm{z} 265.1294$.

(1R,2R)-2-Allyl-2-(6-methoxy-9H-purin-9-yl)cyclopentan-1-ol (4h)

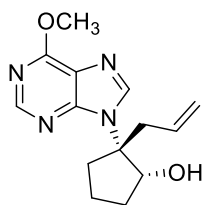

White oil, 43\% yield, 99\% ee; $[\alpha]_{D^{20}}^{20}-34.11\left(\mathrm{c}=0.086, \mathrm{CH}_{2} \mathrm{Cl}_{2}\right) ; \mathbf{H P L C}$ CHIRALCEL OD-H, $n$-hexane/2-propanol $=90 / 10$, flow rate $=0.4 \mathrm{~mL} / \mathrm{min}, \lambda=250 \mathrm{~nm}$, retention time: $26.480 \mathrm{~min}$, 27.697 min; TLC: $R_{\mathrm{f}}=0.26$ (dichloromethane: methanol $\left.=60: 1\right)$ [UV]; ${ }^{1} \mathbf{H}$ NMR $(600 \mathrm{MHz}$, $\left.\mathrm{CDCl}_{3}\right) \delta 8.51(\mathrm{~s}, 1 \mathrm{H}), 7.77(\mathrm{~s}, 1 \mathrm{H}), 6.92-6.91(\mathrm{~m}, 1 \mathrm{H}), 5.46-5.38(\mathrm{~m}, 1 \mathrm{H}), 5.02-5.00(\mathrm{~m}, 1 \mathrm{H})$, 4.82-4.79 (m, 1H), 4.64-4.60 (m, 1H), $4.21(\mathrm{~s}, 3 \mathrm{H}), 2.88-2.85(\mathrm{~m}, 1 \mathrm{H}), 2.49-2.46(\mathrm{~m}, 1 \mathrm{H})$, 2.40-2.30 (m, 2H), 2.13-2.07 (m, 1H), 1.95-1.81 (m, 3H); ${ }^{13} \mathbf{C}$ NMR (150 MHz, CDCl 3$) \delta 161.6$, $151.6,151.1,142.3,131.5,122.3,120.6,79.0,71.2,54.6,35.3,33.3,29.7,19.4$; HRMS (ESI-TOF): exact mass calcd for $\mathrm{C}_{14} \mathrm{H}_{19} \mathrm{~N}_{4} \mathrm{O}_{2}(\mathrm{M}+\mathrm{H})^{+}$requires $\mathrm{m} / \mathrm{z}$ 275.1502, found $\mathrm{m} / \mathrm{z}$ 275.1500

(S)-2-Allyl-2-(6-methoxy-9H-purin-9-yl)cyclopentan-1-one (S-3h)

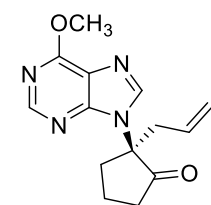

White oil, 45\% yield, 99\% ee; $[\alpha]_{\mathrm{D}}{ }^{20}=9.87\left(\mathrm{c}=0.076, \mathrm{CH}_{2} \mathrm{Cl}_{2}\right)$; HPLC CHIRALCEL OD-H, $n$-hexane/2-propanol $=70 / 30$, flow rate $=0.8 \mathrm{~mL} / \mathrm{min}, \lambda=256 \mathrm{~nm}$, retention time: $7.733 \mathrm{~min}$, 10.490 min; TLC: $R_{\mathrm{f}}=0.31$ (dichloromethane: methanol $\left.=60: 1\right)$ [UV]; ${ }^{1} \mathbf{H}$ NMR $(600 \mathrm{MHz}$, $\left.\mathrm{CDCl}_{3}\right) \delta 8.47(\mathrm{~s}, 1 \mathrm{H}), 8.21(\mathrm{~s}, 1 \mathrm{H}), 5.75-5.68(\mathrm{~m}, 1 \mathrm{H}), 5.16-5.10(\mathrm{~m}, 2 \mathrm{H}), 4.17(\mathrm{~s}, 3 \mathrm{H}), 2.95-2.89$ 
(m, 3H), 2.74-2.64 (m, 2H), 2.56-2.50 (m, 1H), 2.20-2.13 (m, 1H), 2.06-1.99 (m, 1H); ${ }^{13} \mathbf{C}$ NMR $\left(150 \mathrm{MHz}, \mathrm{CDCl}_{3}\right) \delta 213.1,161.3,151.6,140.9,130.2,121.2,68.2,54.3,38.8,36.6,34.4,18.3$; HRMS (ESI-TOF): exact mass calcd for $\mathrm{C}_{14} \mathrm{H}_{17} \mathrm{~N}_{4} \mathrm{O}_{2}(\mathrm{M}+\mathrm{H})^{+}$requires $\mathrm{m} / \mathrm{z}$ 273.1346, found $\mathrm{m} / \mathrm{z}$ 273.1345 .

(3) The analytical and spectral characterization data of derivatives

(1R,2S)-2-(6-Amino-9H-purin-9-yl)cyclopentan-1-ol (5a)

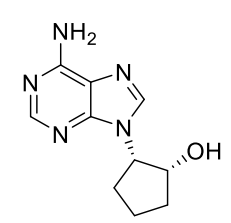

White solid, m.p. $196.7-203.5{ }^{\circ} \mathrm{C}, 15.3 \mathrm{mg}, 70 \%$ yield, $96 \%$ ee; $[\alpha]_{\mathrm{D}}{ }^{25}=-39.86(\mathrm{c}=0.139$, $\mathrm{CH}_{2} \mathrm{Cl}_{2}$ ); HPLC CHIRALCEL ID, $n$-hexane $/ 2$-propanol $=60 / 40$, flow rate $=0.9 \mathrm{~mL} / \mathrm{min}, \lambda=250$ $\mathrm{nm}$, retention time: $17.091 \mathrm{~min}, 42.464 \mathrm{~min}$; TLC: $\mathrm{R}_{\mathrm{f}}=0.26$ (dichloromethane:methanol $=20: 1$ ) [UV]; ${ }^{1} \mathbf{H}$ NMR $\left(400 \mathrm{MHz}, \mathrm{CD}_{3} \mathrm{OD}\right) \delta 8.24(\mathrm{~s}, 1 \mathrm{H}), 8.20(\mathrm{~s}, 1 \mathrm{H}), 4.82-4.76(\mathrm{~m}, 1 \mathrm{H}), 4.34-4.31(\mathrm{~m}$, 1H), 2.40-2.29 (m, 1H), 2.25-2.18 (m, 1H), 2.13-2.01 (m, 2H), 1.89-1.75 (m, 2H); ${ }^{13}$ C NMR (100 $\left.\mathrm{MHz}, \mathrm{CD}_{3} \mathrm{OD}\right) \delta 157.1,153.3,151.0,142.3,119.8,72.5,60.1,33.5,28.6,20.9$; HRMS (ESI-TOF): exact mass calcd for $\mathrm{C}_{10} \mathrm{H}_{14} \mathrm{~N}_{5} \mathrm{O}(\mathrm{M}+\mathrm{H})^{+}$requires m/z 220.1193, found $\mathrm{m} / \mathrm{z} 220.1188$.

9-((1S,2S)-2-Fluorocyclopentyl)-6-methoxy-9H-purine (6a)<smiles>COc1ncnc2c1ncn2C1CCCC1F</smiles>

White solid, m.p. $78.2-80.5{ }^{\circ} \mathrm{C}, 16.8 \mathrm{mg}, 71 \%$ yield, $98 \%$ ee; $[\alpha]_{\mathrm{D}}^{25}=12.60\left(\mathrm{c}=0.320, \mathrm{CH}_{2} \mathrm{Cl}_{2}\right)$; HPLC CHIRALCEL IE, $n$-hexane/2-propanol $=50 / 50$, flow rate $=0.8 \mathrm{~mL} / \mathrm{min}, \lambda=250 \mathrm{~nm}$, retention time: $13.787 \mathrm{~min}, 20.533 \mathrm{~min}$; TLC: $\mathrm{R}_{\mathrm{f}}=0.73$ (dichloromethane:methanol = 30:1) [UV]; ${ }^{1} \mathbf{H}$ NMR $\left(600 \mathrm{MHz}, \mathrm{CDCl}_{3}\right) \delta 8.53(\mathrm{~s}, 1 \mathrm{H}), 7.91(\mathrm{~s}, 1 \mathrm{H}), 5.50-5.39(\mathrm{~m}, 1 \mathrm{H}), 4.97-4.90(\mathrm{~m}, 1 \mathrm{H})$, $4.19(\mathrm{~s}, 3 \mathrm{H}), 2.45-2.40(\mathrm{~m}, 1 \mathrm{H}), 2.35-2.25(\mathrm{~m}, 2 \mathrm{H}), 2.13-1.99(\mathrm{~m}, 3 \mathrm{H}) ;{ }^{13} \mathbf{C}$ NMR (150 MHz, $\left.\mathrm{CDCl}_{3}\right) \delta 161.4,152.1,151.98,141.2,122.4,98.3(J=181.5 \mathrm{~Hz}), 62.8(J=27.0 \mathrm{~Hz}), 54.4,31.4(J$ $=21.0 \mathrm{~Hz}), 30.0(J=3.0 \mathrm{~Hz}), 21.9(J=1.5 \mathrm{~Hz})$; HRMS (ESI-TOF): exact mass calcd for $\mathrm{C}_{11} \mathrm{H}_{14} \mathrm{FN}{ }_{4} \mathrm{O}(\mathrm{M}+\mathrm{H})^{+}$requires $\mathrm{m} / \mathrm{z} 237.1147$, found $\mathrm{m} / \mathrm{z} 237.1149$.

(1R,2S)-2-(6-Methoxy-9H-purin-9-yl)cyclopentyl methanesulfonate (7a) 


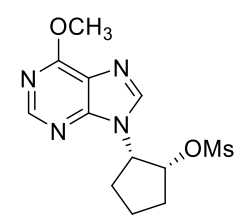

White solid, m.p. $130.5-141.7{ }^{\circ} \mathrm{C}, 151.4 \mathrm{mg}, 97 \%$ yield, $98 \%$ ee; $[\alpha]_{\mathrm{D}}^{20}=-133.85(\mathrm{c}=0.130$, $\mathrm{CH}_{2} \mathrm{Cl}_{2}$ ); HPLC CHIRALCEL OD-H, $n$-hexane $/ 2$-propanol $=80 / 20$, flow rate $=0.8 \mathrm{~mL} / \mathrm{min}, \lambda=$ $250 \mathrm{~nm}$, retention time: $31.213 \mathrm{~min}, 44.790 \mathrm{~min}$; $\mathbf{T L C}: \mathrm{R}_{\mathrm{f}}=0.45$ (dichloromethane: $\mathrm{methanol}=$ 30:1) [UV]; ${ }^{1} \mathbf{H}$ NMR (600 MHz, $\left.\mathrm{CDCl}_{3}\right) \delta 8.55(\mathrm{~s}, 1 \mathrm{H}), 8.12(\mathrm{~s}, 1 \mathrm{H}), 5.30-5.28(\mathrm{~m}, 1 \mathrm{H})$, 5.06-5.02 (m, 1H), $4.19(\mathrm{~s}, 3 \mathrm{H}), 2.52(\mathrm{~s}, 3 \mathrm{H}), 2.46-2.38(\mathrm{~m}, 2 \mathrm{H}), 2.33-2.28(\mathrm{~m}, 2 \mathrm{H}), 2.22-2.15(\mathrm{~m}$, 1H), 1.94-1.86 (m, 1H); ${ }^{13} \mathbf{C}$ NMR (150 MHz, $\left.\mathrm{CDCl}_{3}\right) \delta 161.3,152.4,152.2,141.1,121.3,81.6$, 57.4, 54.5, 38.0, 31.4, 27.4, 19.9; HRMS (ESI-TOF): exact mass calcd for $\mathrm{C}_{12} \mathrm{H}_{17} \mathrm{~N}_{4} \mathrm{O}_{4} \mathrm{~S}(\mathrm{M}+\mathrm{H})^{+}$ requires $\mathrm{m} / \mathrm{z} 313.0965$, found $\mathrm{m} / \mathrm{z} 313.0965$.

\section{S-((1S,2S)-2-(6-Methoxy-9H-purin-9-yl)cyclopentyl) ethanethioate (8a)}

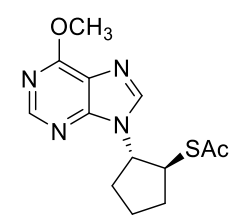

White oil, $22.2 \mathrm{mg}, 76 \%$ yield, $98 \%$ ee; $[\alpha]_{\mathrm{D}}{ }^{25}=-22.74\left(\mathrm{c}=0.236, \mathrm{CH}_{2} \mathrm{Cl}_{2}\right) ;$ HPLC CHIRALCEL OD-H, $n$-hexane/2-propanol $=80 / 20$, flow rate $=0.8 \mathrm{~mL} / \mathrm{min}, \lambda=250 \mathrm{~nm}$, retention time: 10.171 min, 13.903 min; TLC: $R_{\mathrm{f}}=0.41$ (dichloromethane:methanol = 30:1) [UV]; ${ }^{1} \mathbf{H} \mathbf{N M R}(600 \mathrm{MHz}$, $\left.\mathrm{CDCl}_{3}\right) \delta 8.53(\mathrm{~s}, 1 \mathrm{H}), 7.95(\mathrm{~s}, 1 \mathrm{H}), 4.78(\mathrm{q}, J=9.0 \mathrm{~Hz}, 1 \mathrm{H}), 4.25(\mathrm{q}, J=9.0 \mathrm{~Hz}, 1 \mathrm{H}), 4.18(\mathrm{~s}, 3 \mathrm{H})$, 2.51-2.45 (m, 1H), 2.39-2.28 (m, 2H), $2.19(\mathrm{~s}, 3 \mathrm{H}), 2.13-2.09(\mathrm{~m}, 1 \mathrm{H}), 1.97-1.92(\mathrm{~m}, 1 \mathrm{H})$, 1.85-1.78 (m, 1H); ${ }^{13} \mathbf{C}$ NMR $\left(150 \mathrm{MHz}, \mathrm{CDCl}_{3}\right) \delta 194.8,161.2,152.2,151.9,141.2,122.1,61.6$, 54.3, 47.0, 31.5, 31.4, 30.6, 22.5; HRMS (ESI-TOF): exact mass calcd for $\mathrm{C}_{13} \mathrm{H}_{17} \mathrm{~N}_{4} \mathrm{O}_{2} \mathrm{~S}(\mathrm{M}+\mathrm{H})^{+}$ requires $\mathrm{m} / \mathrm{z} 293.1067$, found $\mathrm{m} / \mathrm{z} 293.1071$.

\section{9-((1S,2S)-2-Azidocyclopentyl)-6-methoxy-9H-purine (9a)}

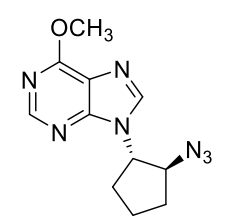

Yellow oil, $67.6 \mathrm{mg}, 87 \%$ yield, $97 \%$ ee; $[\alpha]_{\mathrm{D}}{ }^{20}=46.35\left(\mathrm{c}=0.320, \mathrm{CH}_{2} \mathrm{Cl}_{2}\right) ;$ HPLC CHIRALCEL ID, $n$-hexane/2-propanol $=70 / 30$, flow rate $=0.8 \mathrm{~mL} / \mathrm{min}, \lambda=250 \mathrm{~nm}$, retention time: $15.488 \mathrm{~min}$, 17.397 min; TLC: $\mathrm{R}_{\mathrm{f}}=0.75$ (dichloromethane:methanol = 30:1) [UV]; ${ }^{1} \mathbf{H}$ NMR $(600 \mathrm{MHz}$, $\left.\mathrm{CD}_{3} \mathrm{OD}\right) \delta 8.52(\mathrm{~s}, 1 \mathrm{H}), 8.38(\mathrm{~s}, 1 \mathrm{H}), 4.81(\mathrm{q}, J=8.4 \mathrm{~Hz}, 1 \mathrm{H}), 4.54(\mathrm{q}, J=7.8 \mathrm{~Hz}, 1 \mathrm{H}), 4.18(\mathrm{~s}$, 
3H), 2.37-2.29 (m, 3H), 2.10-2.06 (m, 1H), 1.98-1.92 (m, 1H), 1.87-1.82 (m, 1H); ${ }^{13}$ C NMR $(150$ $\left.\mathrm{MHz}, \mathrm{CD}_{3} \mathrm{OD}\right) \delta 162.3,153.1,143.7,122.5,66.8,63.3,54.8,30.4,30.3,21.7$; HRMS (ESI-TOF): exact mass calcd for $\mathrm{C}_{11} \mathrm{H}_{14} \mathrm{~N}_{7} \mathrm{O}(\mathrm{M}+\mathrm{H})^{+}$requires $\mathrm{m} / \mathrm{z}$ 260.1254, found $\mathrm{m} / \mathrm{z} 260.1257$.

6-Methoxy-9-((1S,2S)-2-(4-phenyl-4,5-dihydro-1H-1,2,3-triazol-1-yl)cyclopentyl)-9H-purine

(10a)

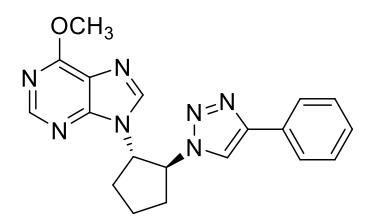

White oil, $33.2 \mathrm{mg}, 92 \%$ yield, $98 \%$ ee; $[\alpha]_{\mathrm{D}}{ }^{25}=222.22\left(\mathrm{c}=0.072, \mathrm{CH}_{2} \mathrm{Cl}_{2}\right) ;$ HPLC CHIRALCEL OD-H, $n$-hexane/2-propanol $=60 / 40$, flow rate $=0.8 \mathrm{~mL} / \mathrm{min}, \lambda=250 \mathrm{~nm}$, retention time: $15.358 \mathrm{~min}, 20.422 \mathrm{~min}$; TLC: $\mathrm{R}_{\mathrm{f}}=0.53$ (dichloromethane:methanol $=30: 1$ ) $[\mathrm{UV}] ;{ }^{1} \mathbf{H}$ NMR $\left(600 \mathrm{MHz}, \mathrm{CDCl}_{3}\right) \delta 8.54(\mathrm{~s}, 1 \mathrm{H}), 7.92(\mathrm{~s}, 1 \mathrm{H}), 7.73(\mathrm{~d}, J=7.8 \mathrm{~Hz}, 2 \mathrm{H}), 7.62(\mathrm{~s}, 1 \mathrm{H}), 7.38$ (t, $J=7.8 \mathrm{~Hz}, 2 \mathrm{H}), 7.31(\mathrm{t}, J=7.8 \mathrm{~Hz}, 1 \mathrm{H}), 5.74-5.70(\mathrm{~m}, 1 \mathrm{H}), 5.47-5.42(\mathrm{~m}, 1 \mathrm{H}), 4.18(\mathrm{~s}, 3 \mathrm{H})$, 2.68-2.64 (m, 2H), 2.51-2.43 (m, 2H), 2.30-2.21 (m, 2H); ${ }^{13} \mathbf{C}$ NMR (150 MHz, $\left.\mathrm{CDCl}_{3}\right) \delta 161.4$, $152.1,148.0,142.3,130.3,129.0,128.4,125.8,119.8,64.4,62.8,54.5,31.6,29.8,21.7$; HRMS (ESI-TOF): exact mass calcd for $\mathrm{C}_{19} \mathrm{H}_{22} \mathrm{~N}_{7} \mathrm{O}(\mathrm{M}+\mathrm{H})^{+}$requires m/z 362.1724, found $\mathrm{m} / \mathrm{z} 362.1718$. (1S,2S)-2-(6-Methoxy-9H-purin-9-yl)cyclopentan-1-amine (11a)

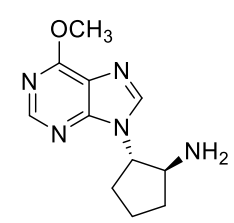

Yellow oil, $20.0 \mathrm{mg}, 86 \%$ yield, $97 \%$ ee; $[\alpha]_{\mathrm{D}}^{25}=34.18\left(\mathrm{c}=0.058, \mathrm{CH}_{2} \mathrm{Cl}_{2}\right) ;$ HPLC CHIRALCEL OD-H, $n$-hexane $/ 2$-propanol $=70 / 30$, flow rate $=0.35 \mathrm{~mL} / \mathrm{min}, \lambda=250 \mathrm{~nm}$, retention time: 26.492 $\min , 30.286 \min$; TLC: $R_{\mathrm{f}}=0.16$ (dichloromethane:methanol $\left.=20: 1\right)[\mathrm{UV}] ;{ }^{1} \mathbf{H}$ NMR $(600 \mathrm{MHz}$, $\left.\mathrm{CD}_{3} \mathrm{OD}\right) \delta 8.51(\mathrm{~s}, 1 \mathrm{H}), 8.35(\mathrm{~s}, 1 \mathrm{H}), 4.61(\mathrm{q}, J=8.4 \mathrm{~Hz}, 1 \mathrm{H}), 4.18(\mathrm{~s}, 3 \mathrm{H}), 3.82(\mathrm{q}, J=8.4 \mathrm{~Hz}$, $1 \mathrm{H}), 2.35-2.23(\mathrm{~m}, 3 \mathrm{H}), 2.03-1.93(\mathrm{~m}, 2 \mathrm{H}), 1.67-1.62(\mathrm{~m}, 1 \mathrm{H}) ;{ }^{13} \mathbf{C} \mathbf{N M R}\left(150 \mathrm{MHz}, \mathrm{CD}_{3} \mathrm{OD}\right) \delta$ 160.8, 152.0, 151.5, 142.2, 121.1, 64.0, 57.1, 53.4, 32.2, 29.8, 20.3; HRMS (ESI-TOF): exact mass calcd for $\mathrm{C}_{11} \mathrm{H}_{16} \mathrm{~N}_{5} \mathrm{O}(\mathrm{M}+\mathrm{H})^{+}$requires $\mathrm{m} / \mathrm{z} 234.1349$, found $\mathrm{m} / \mathrm{z} 234.1348$. 


\section{Copies of ${ }^{1} \mathrm{H}$ and ${ }^{13} \mathrm{C}$ NMR spectra}

(1) Copies of NMR spectra of starting materials

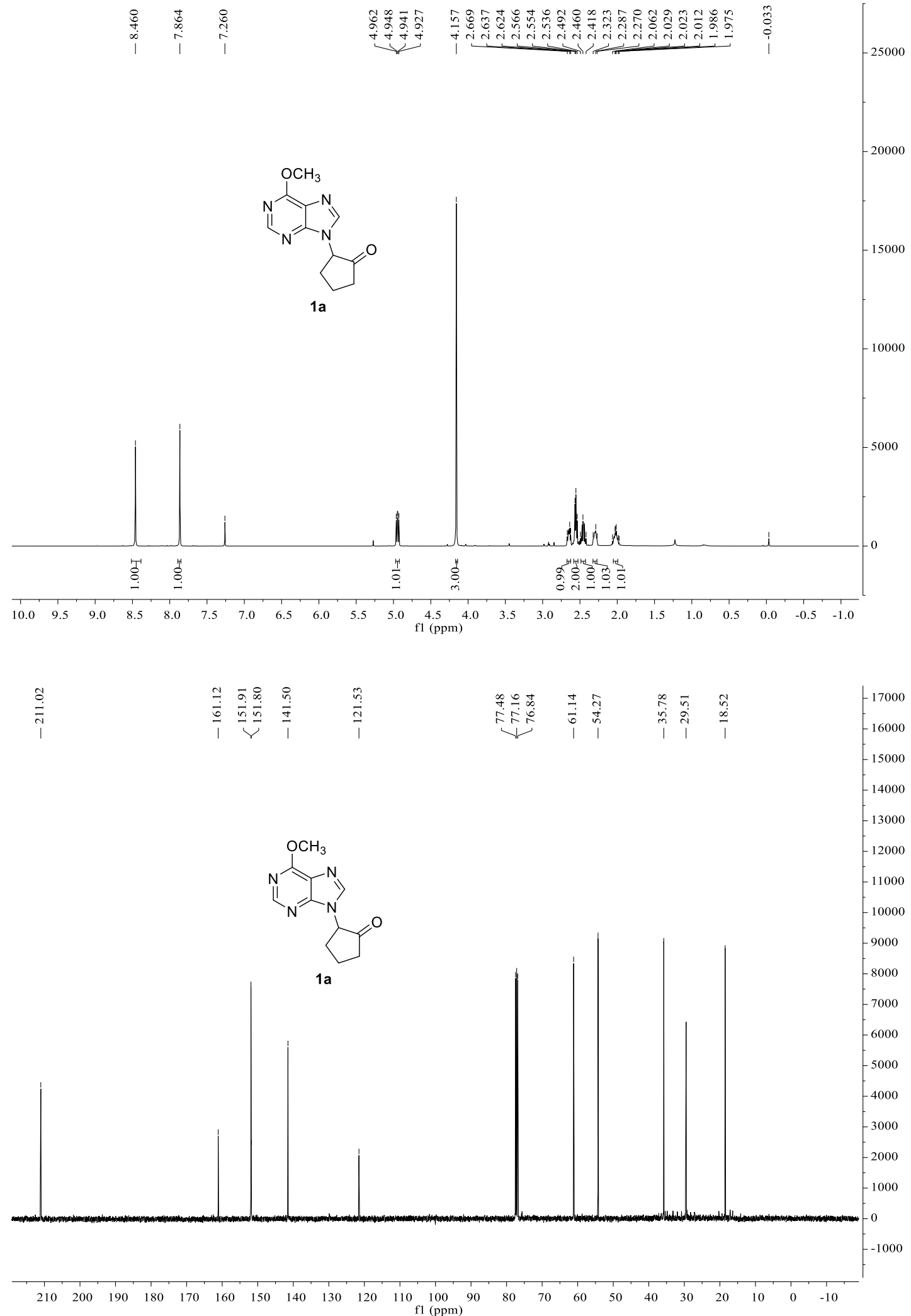



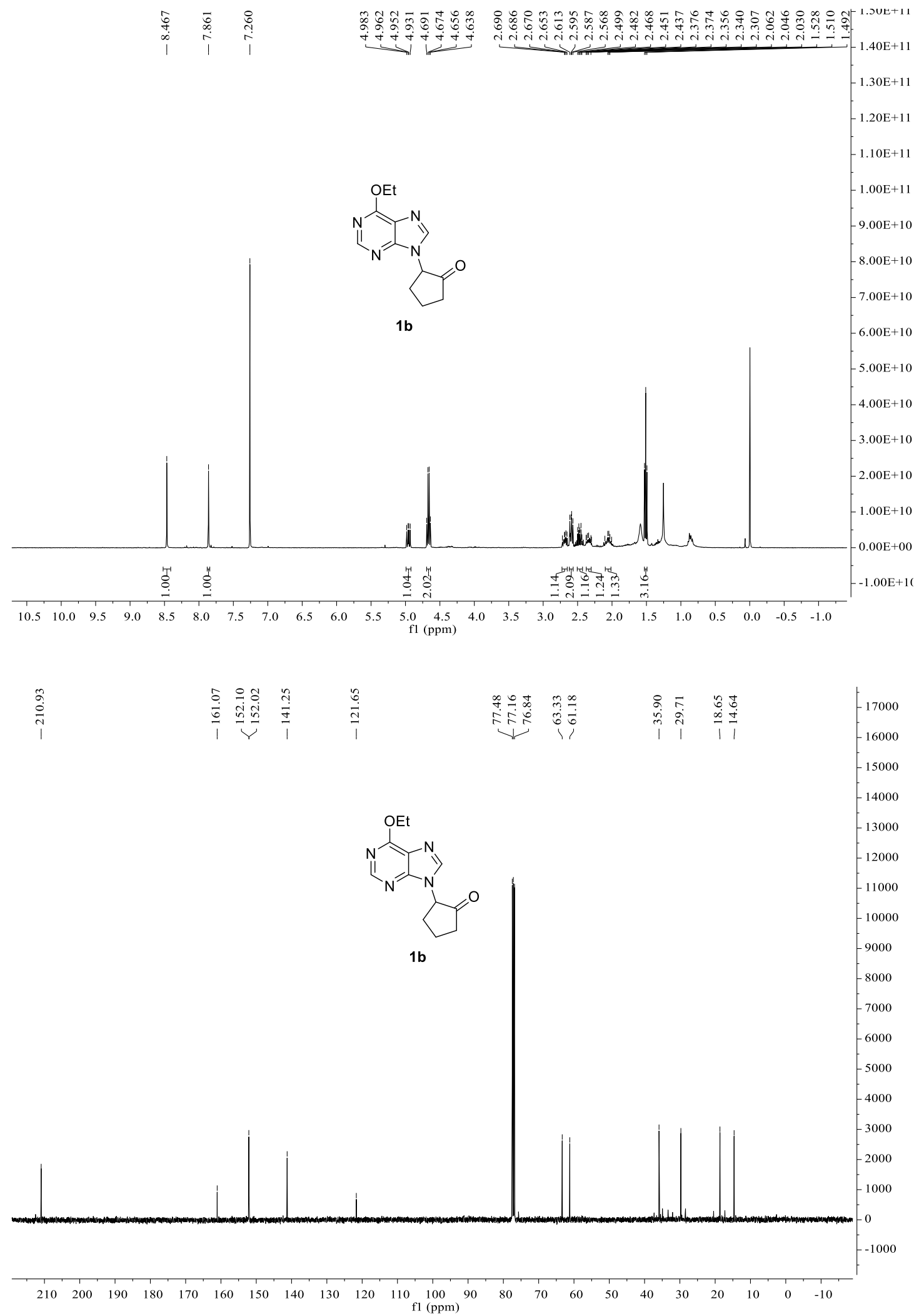


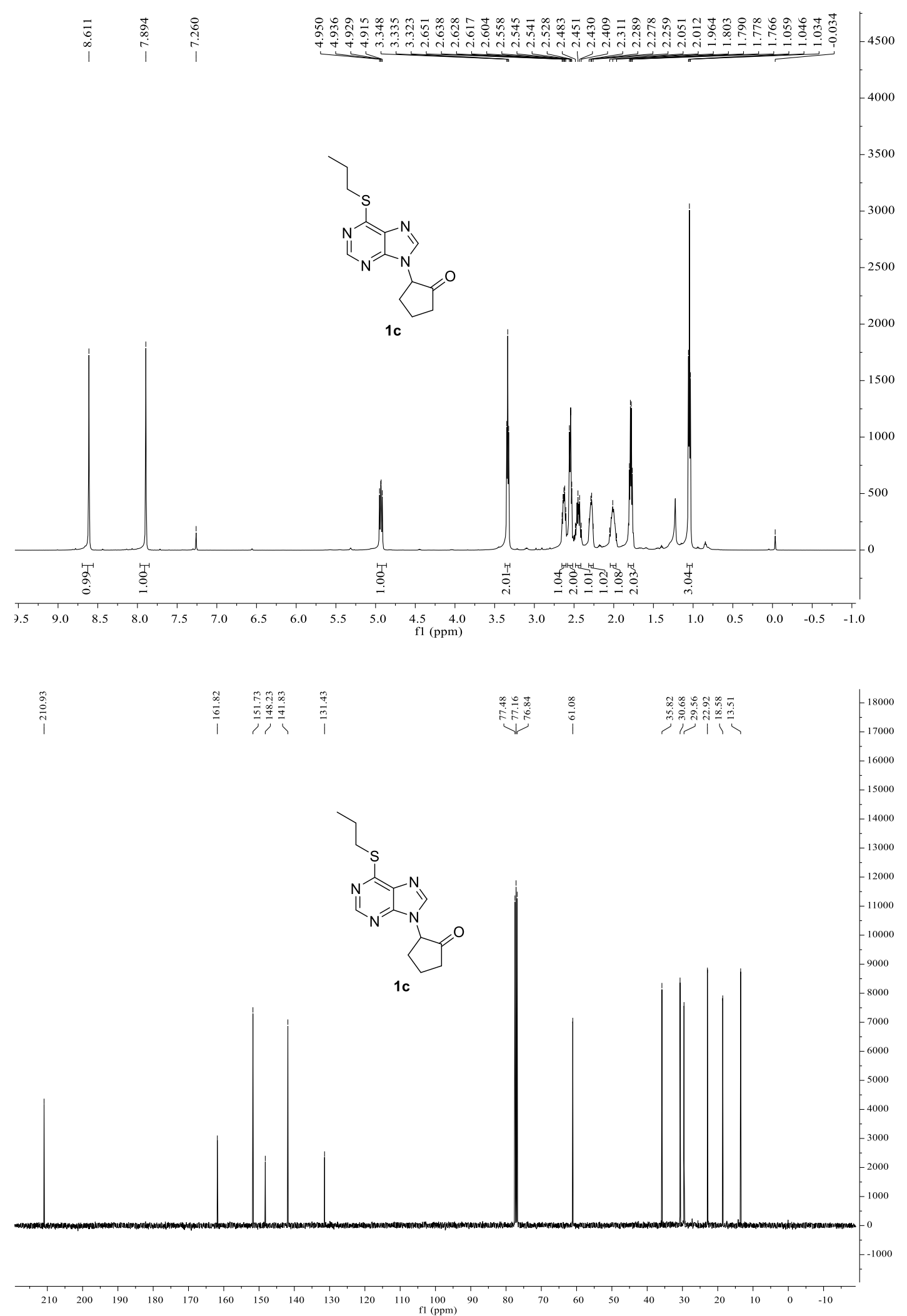




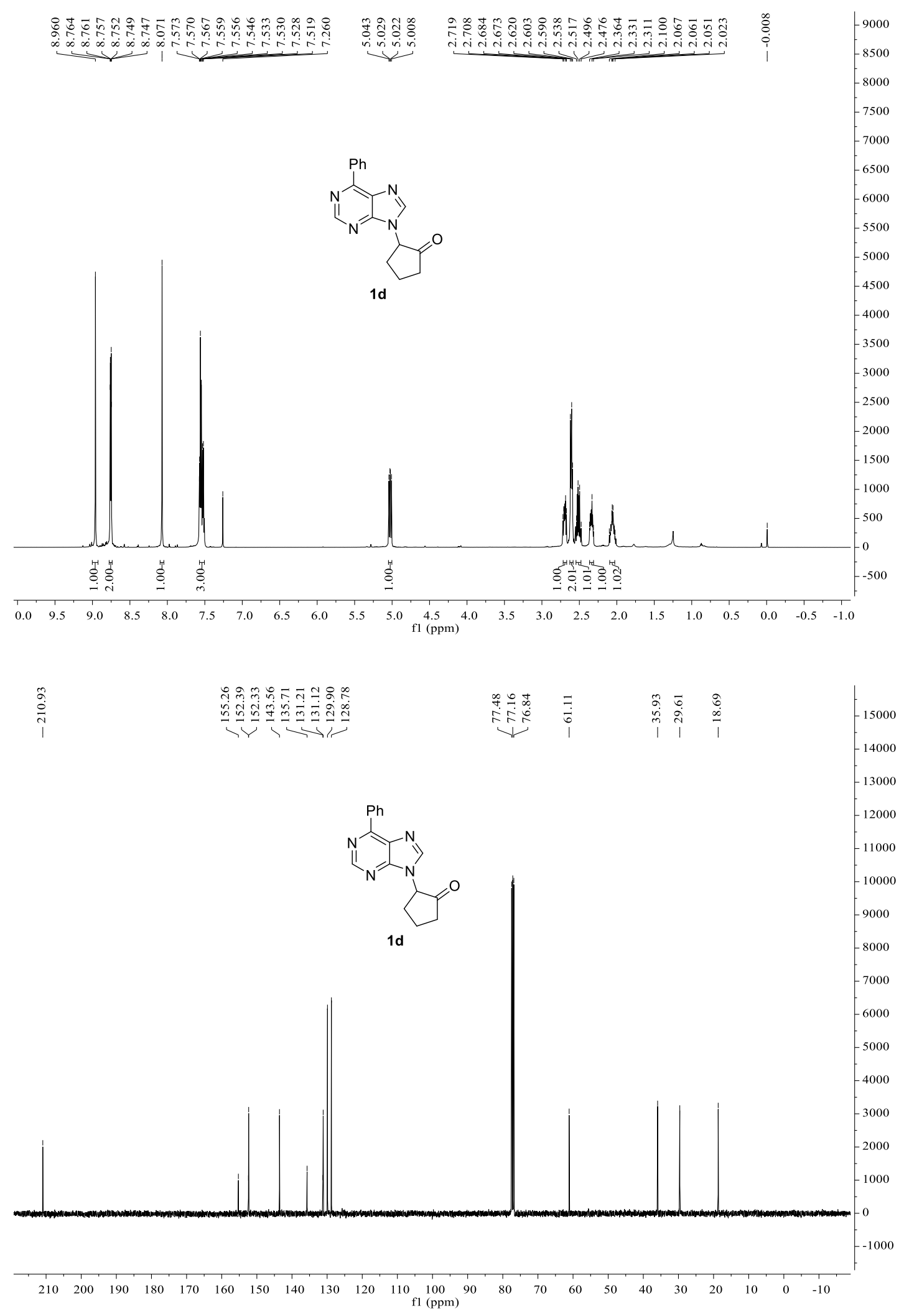




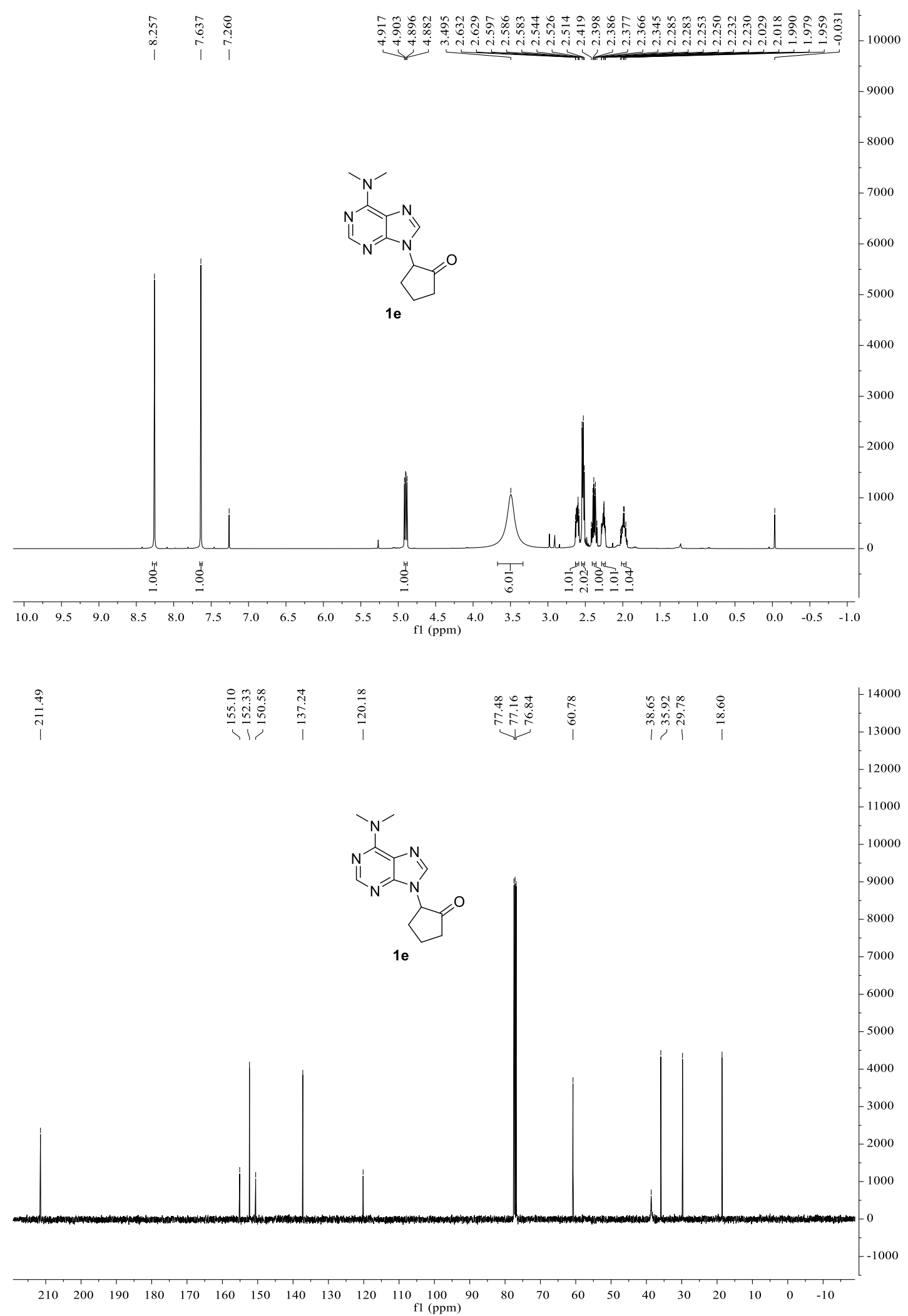



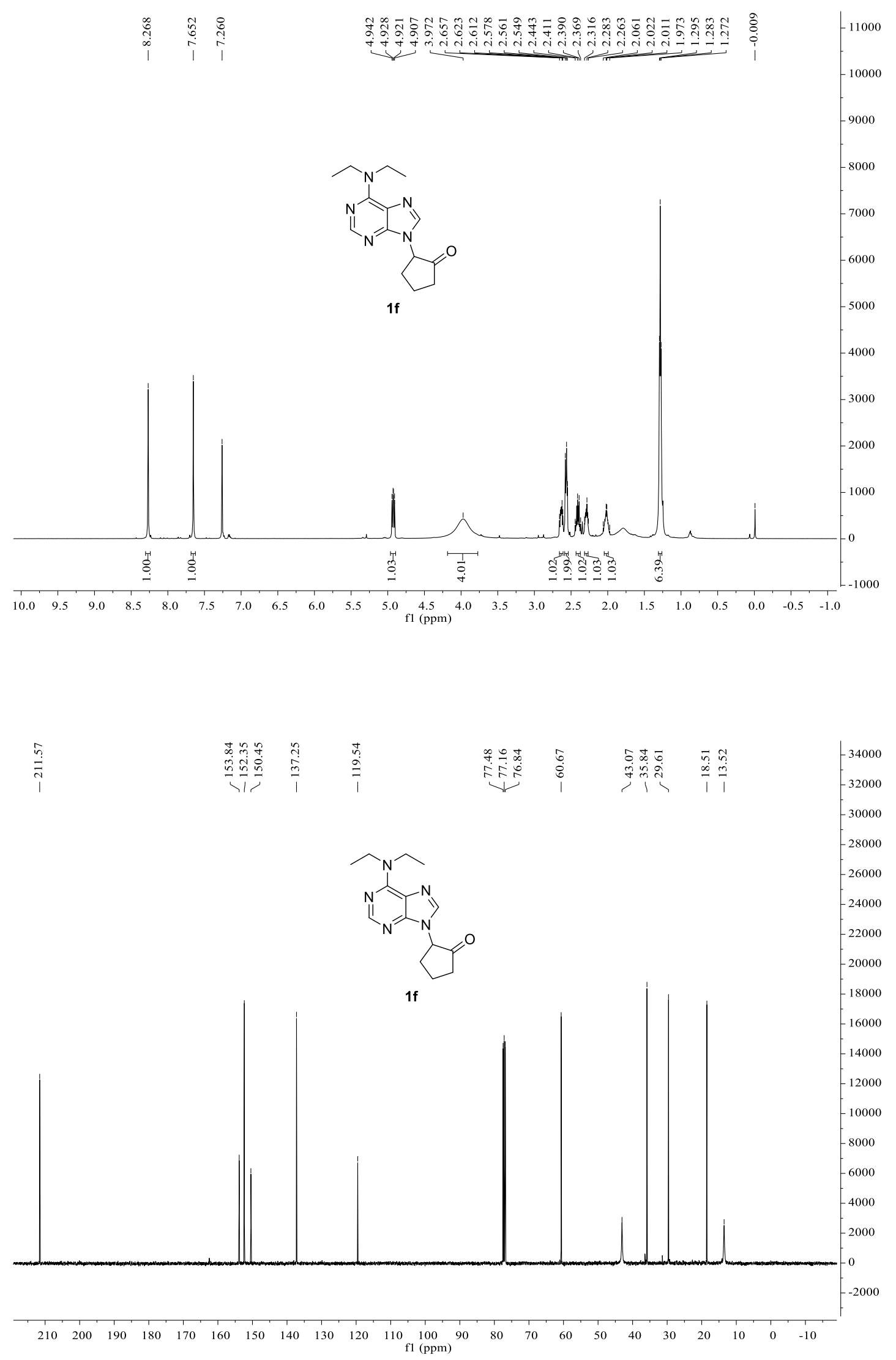


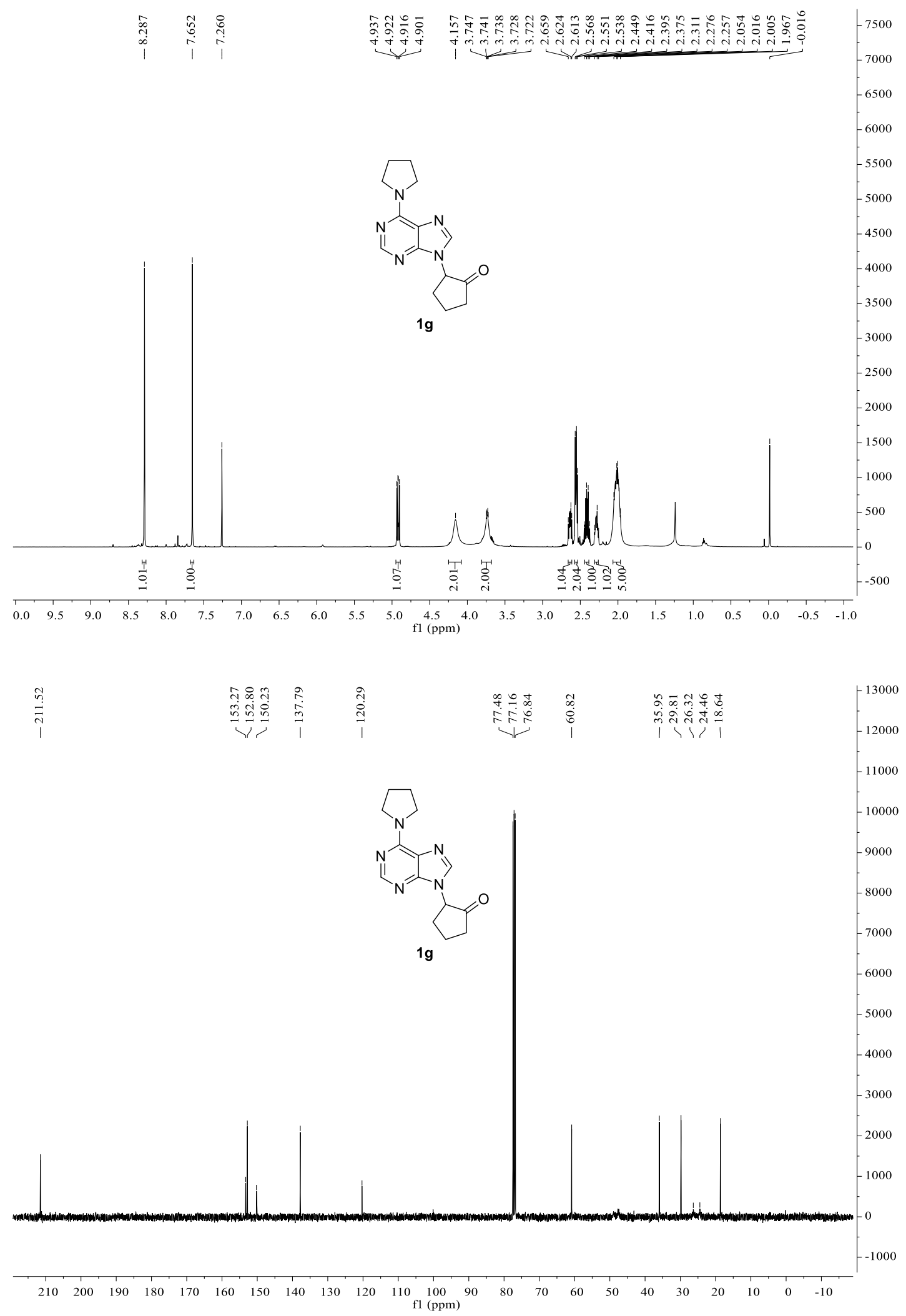




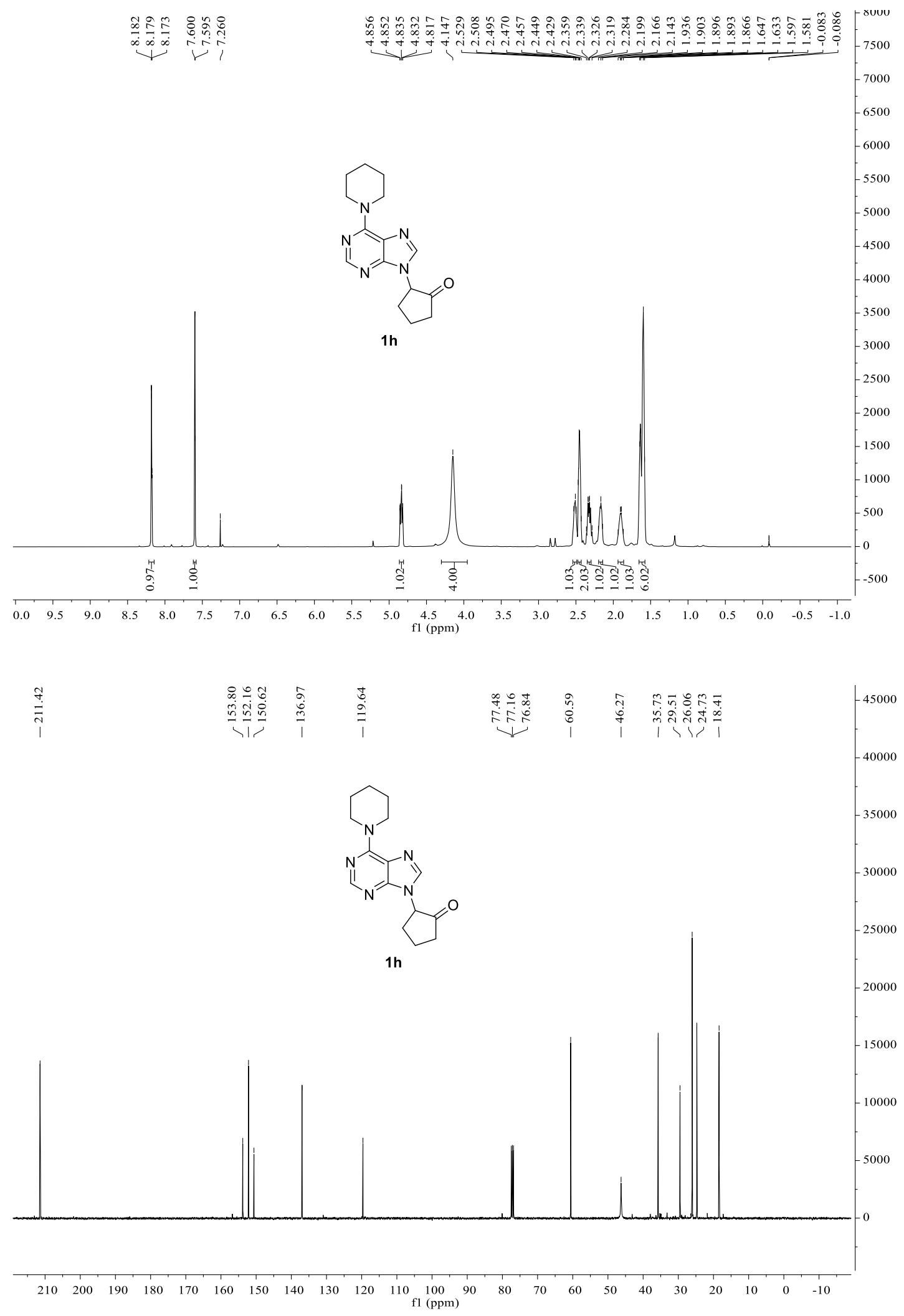




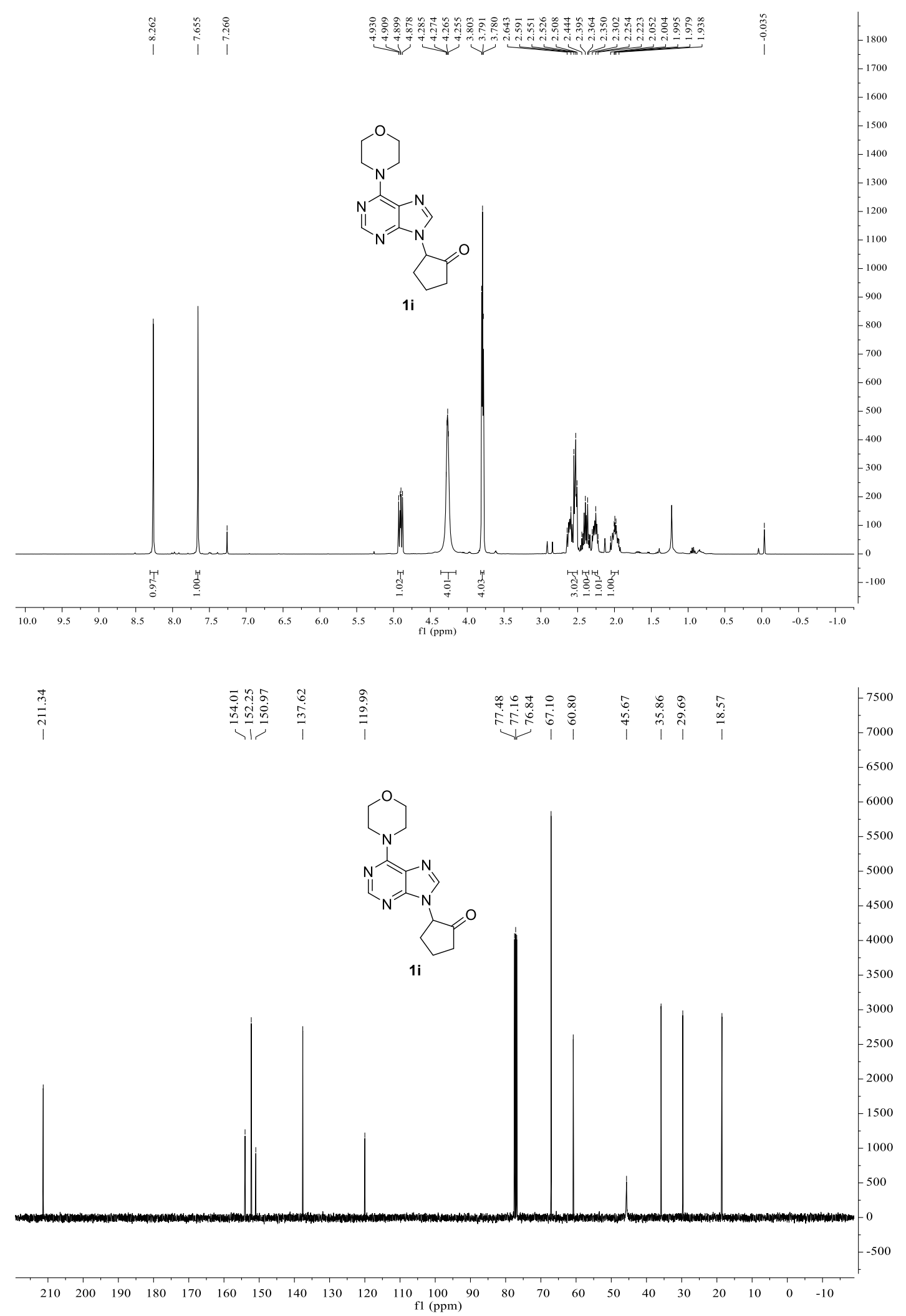



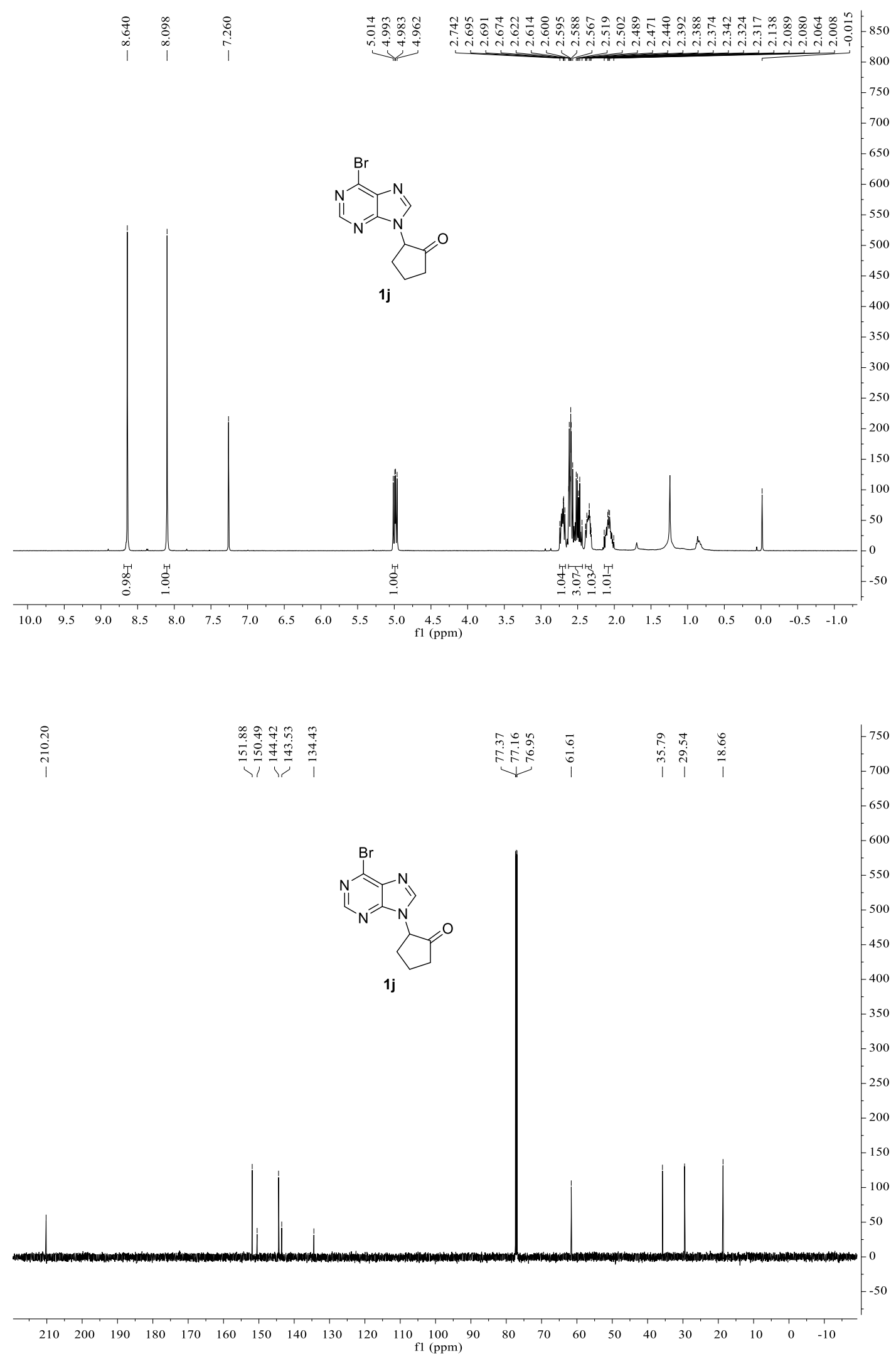


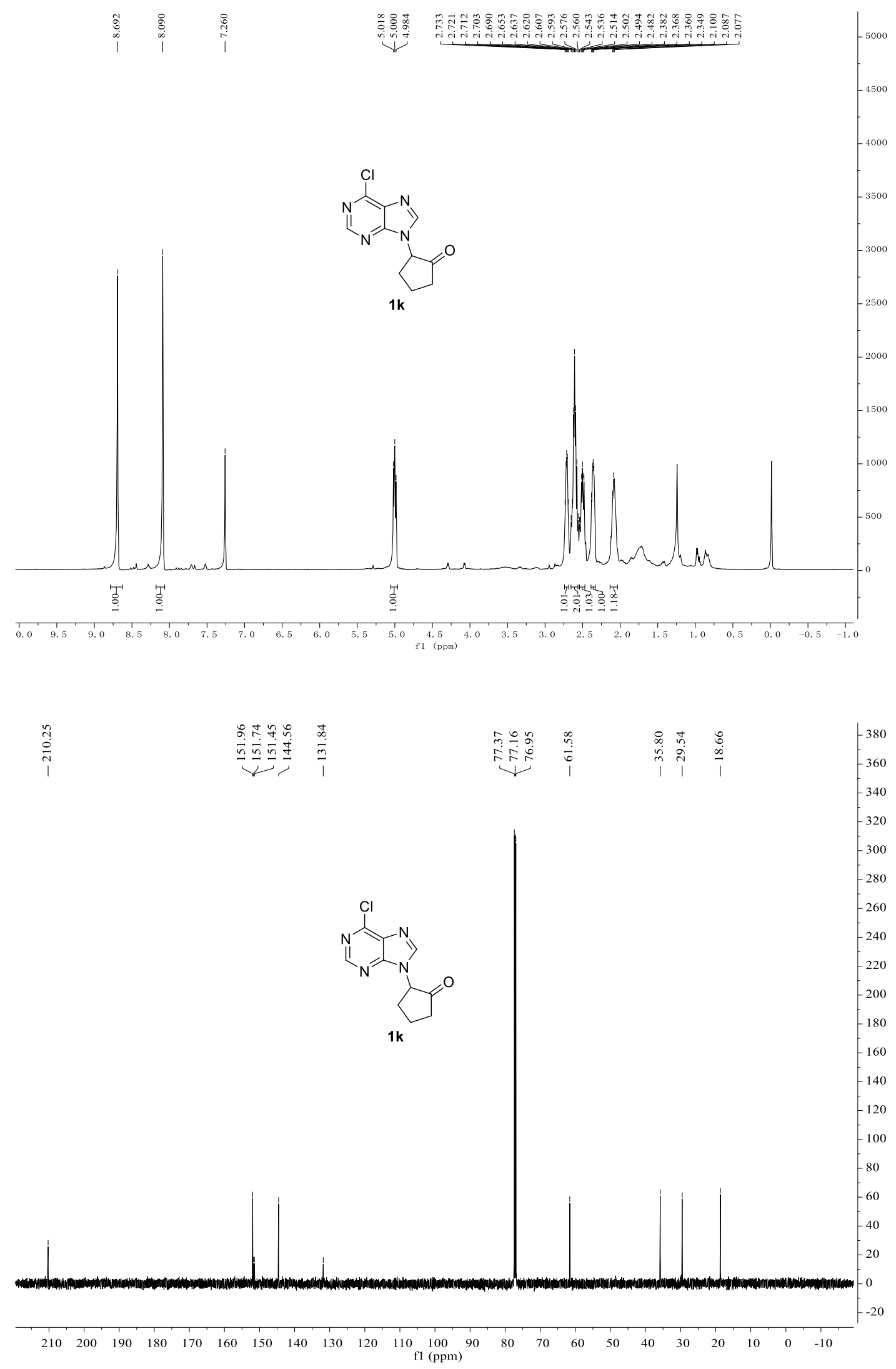




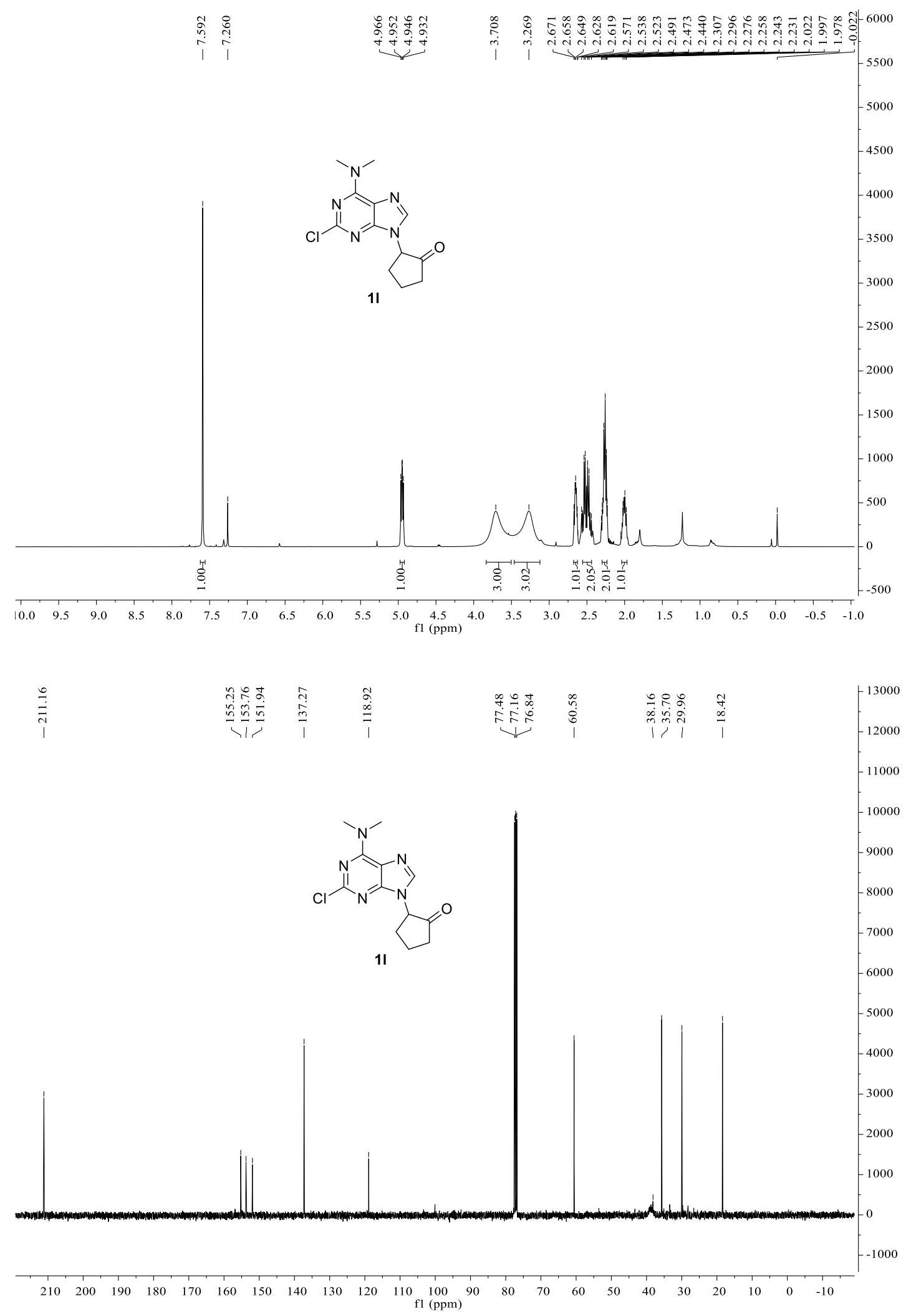



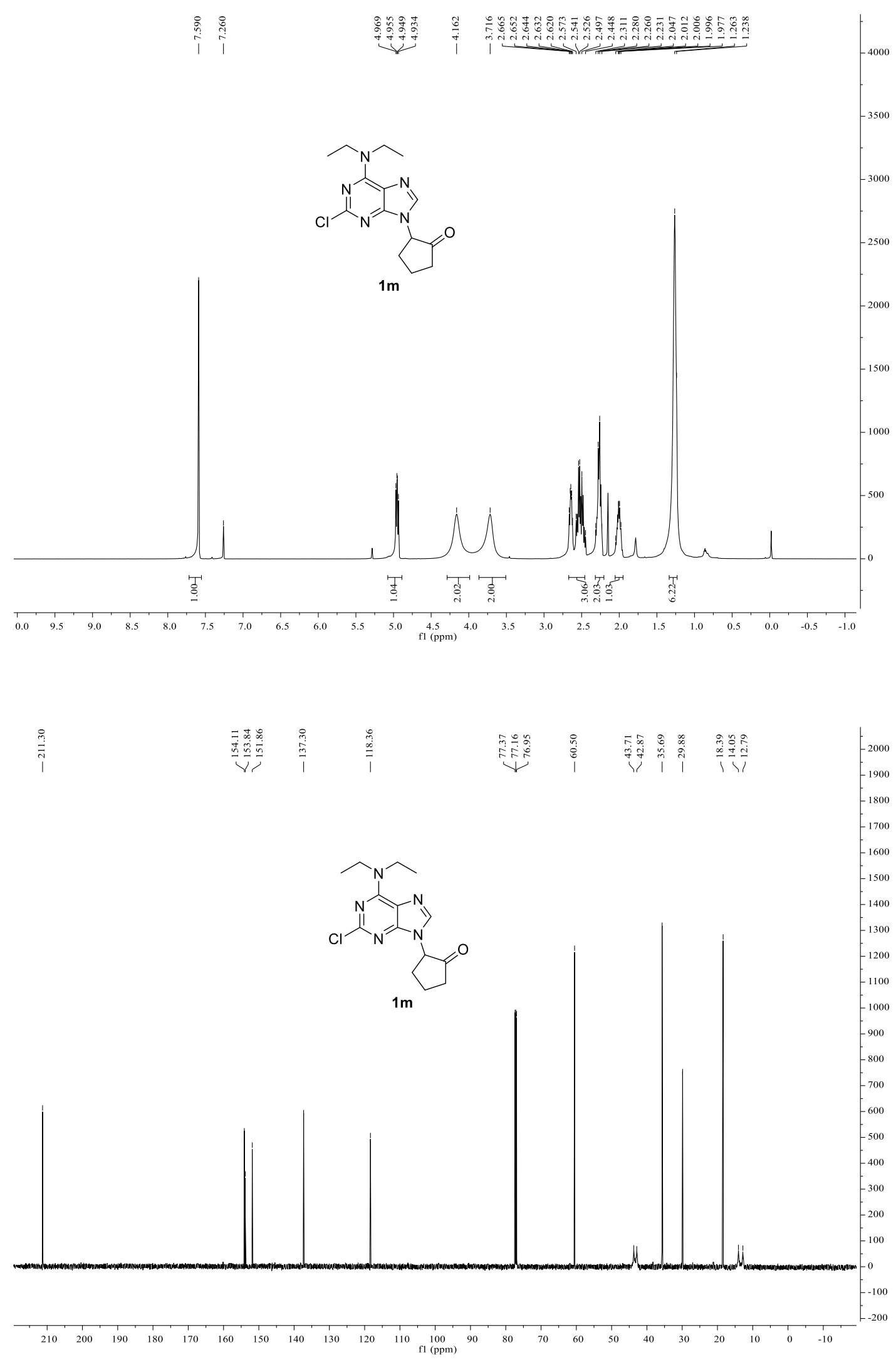


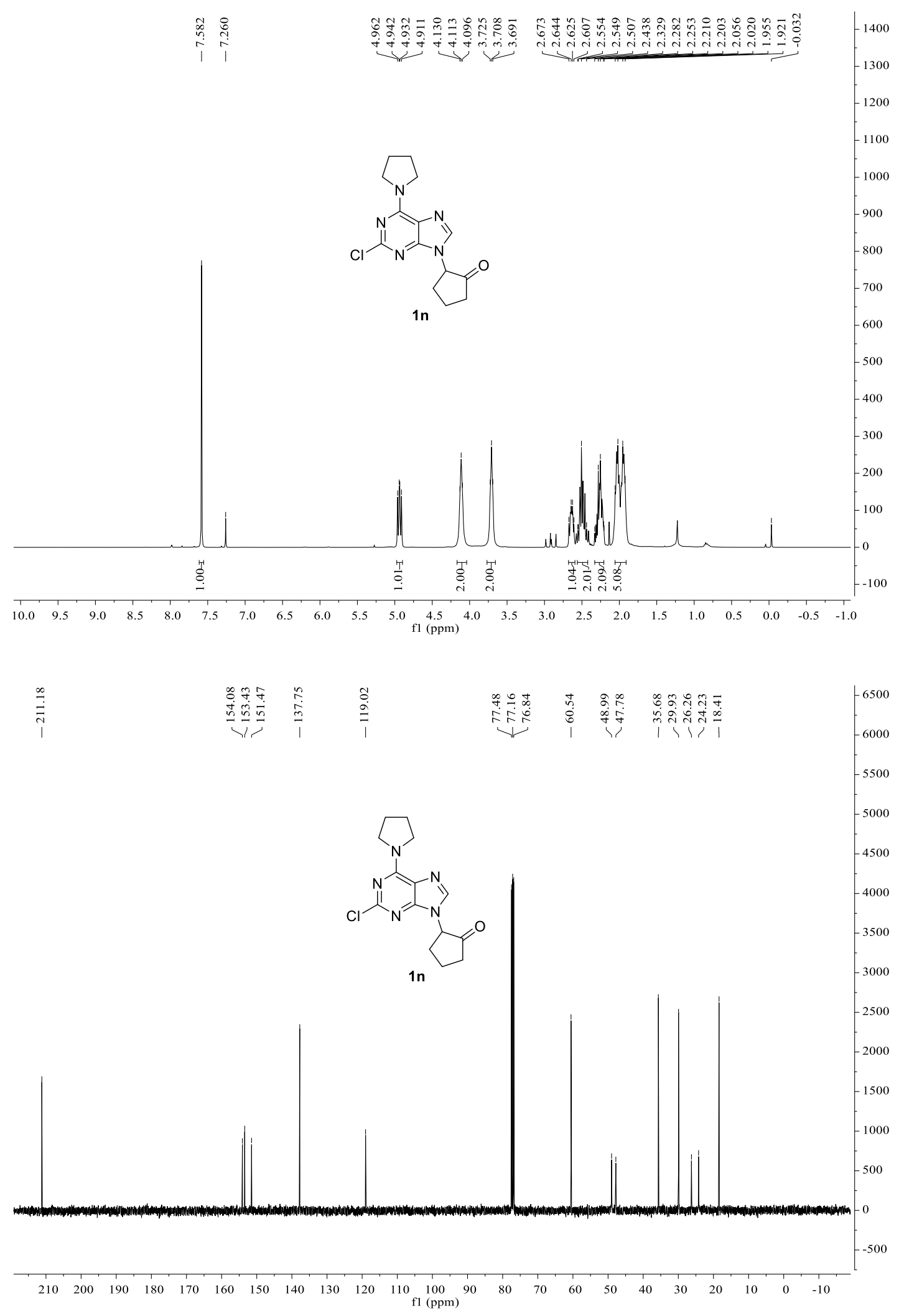



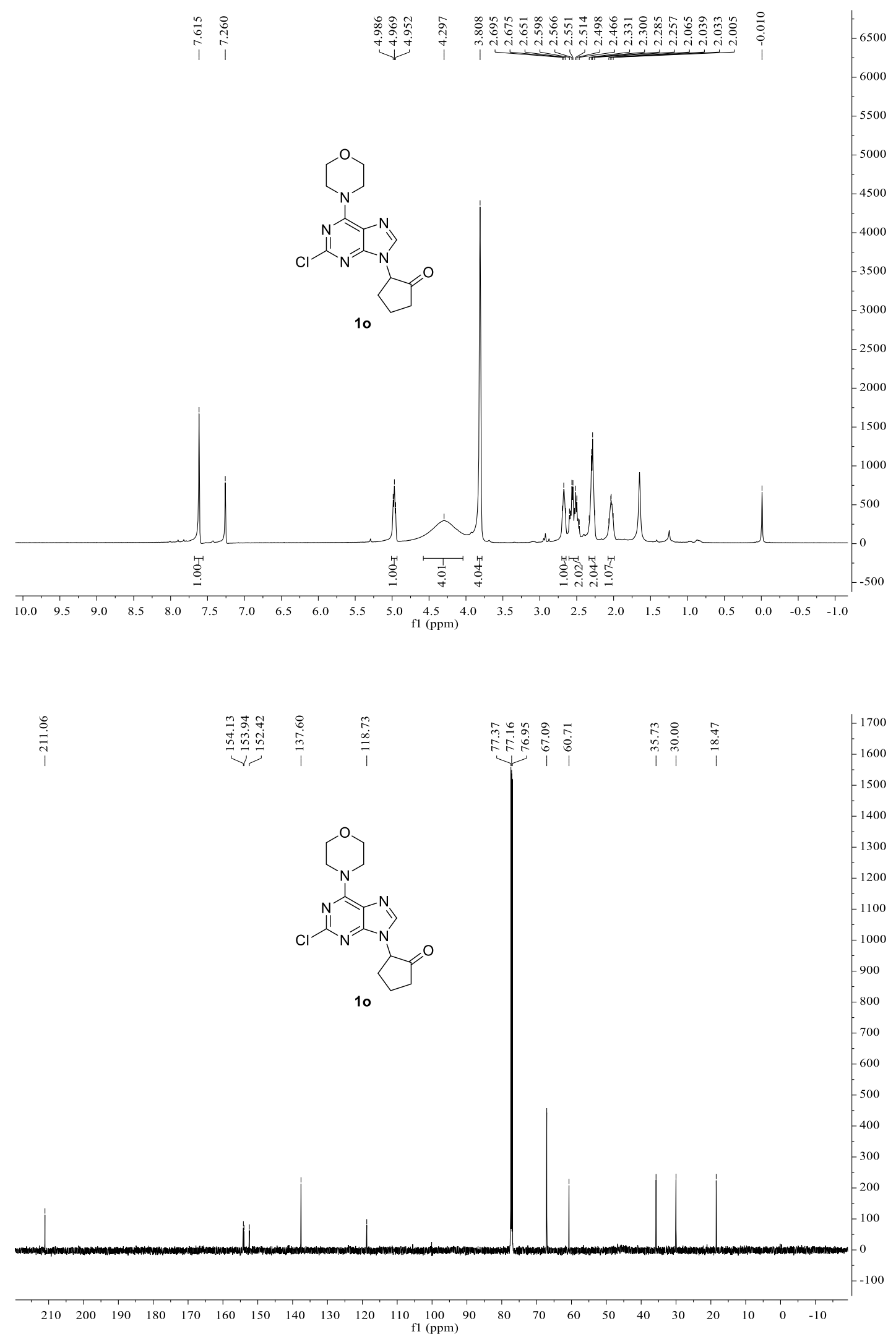


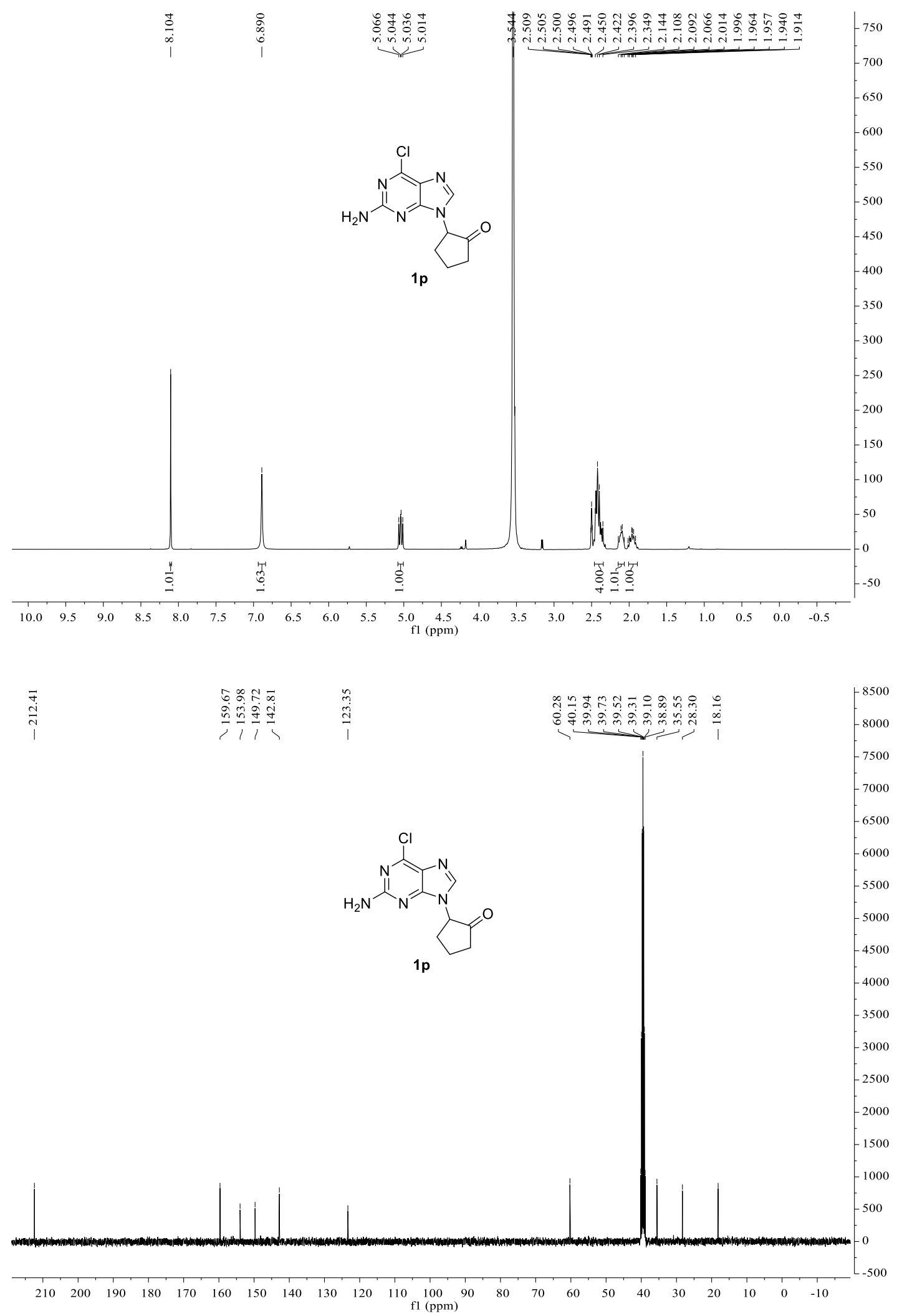




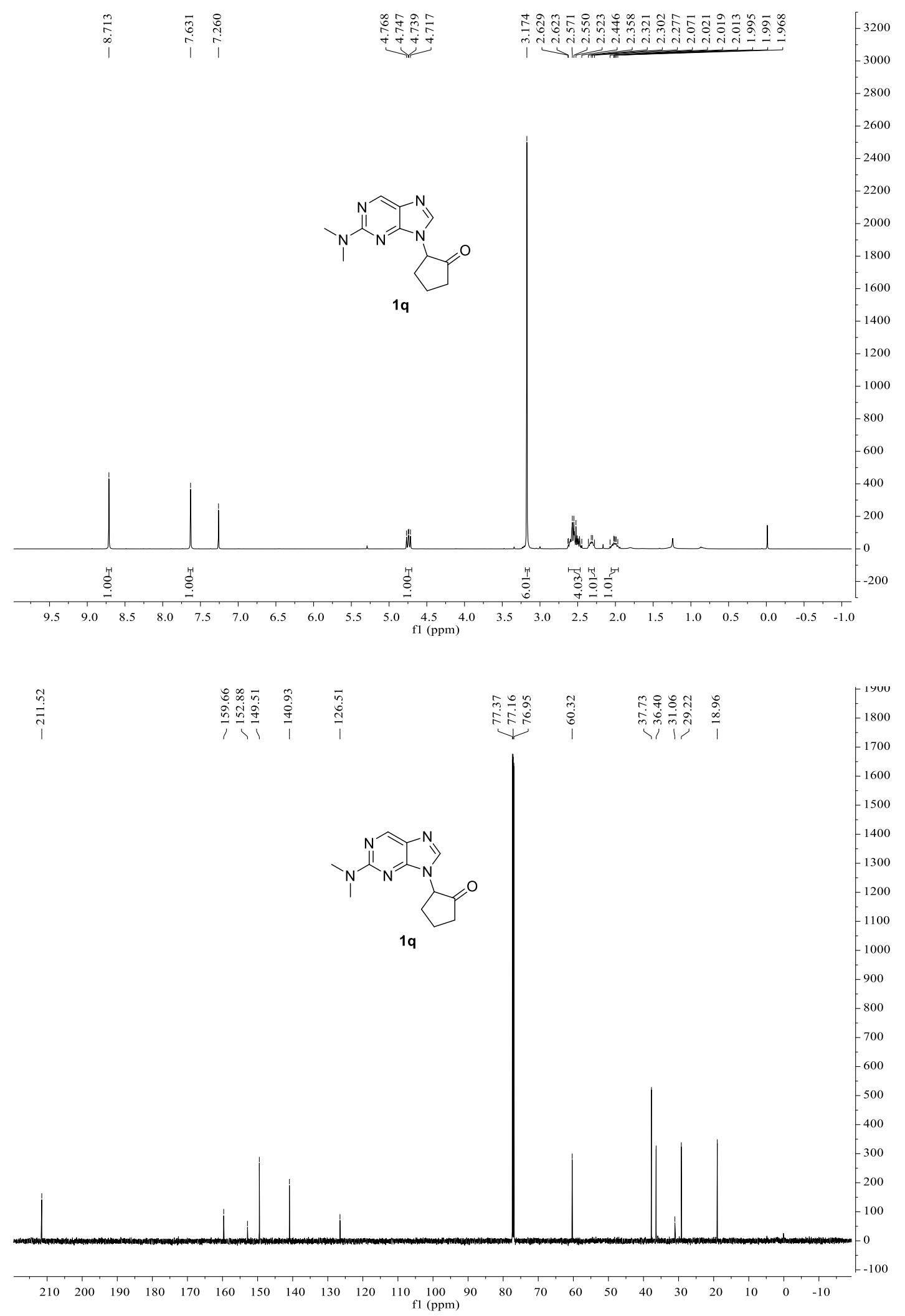




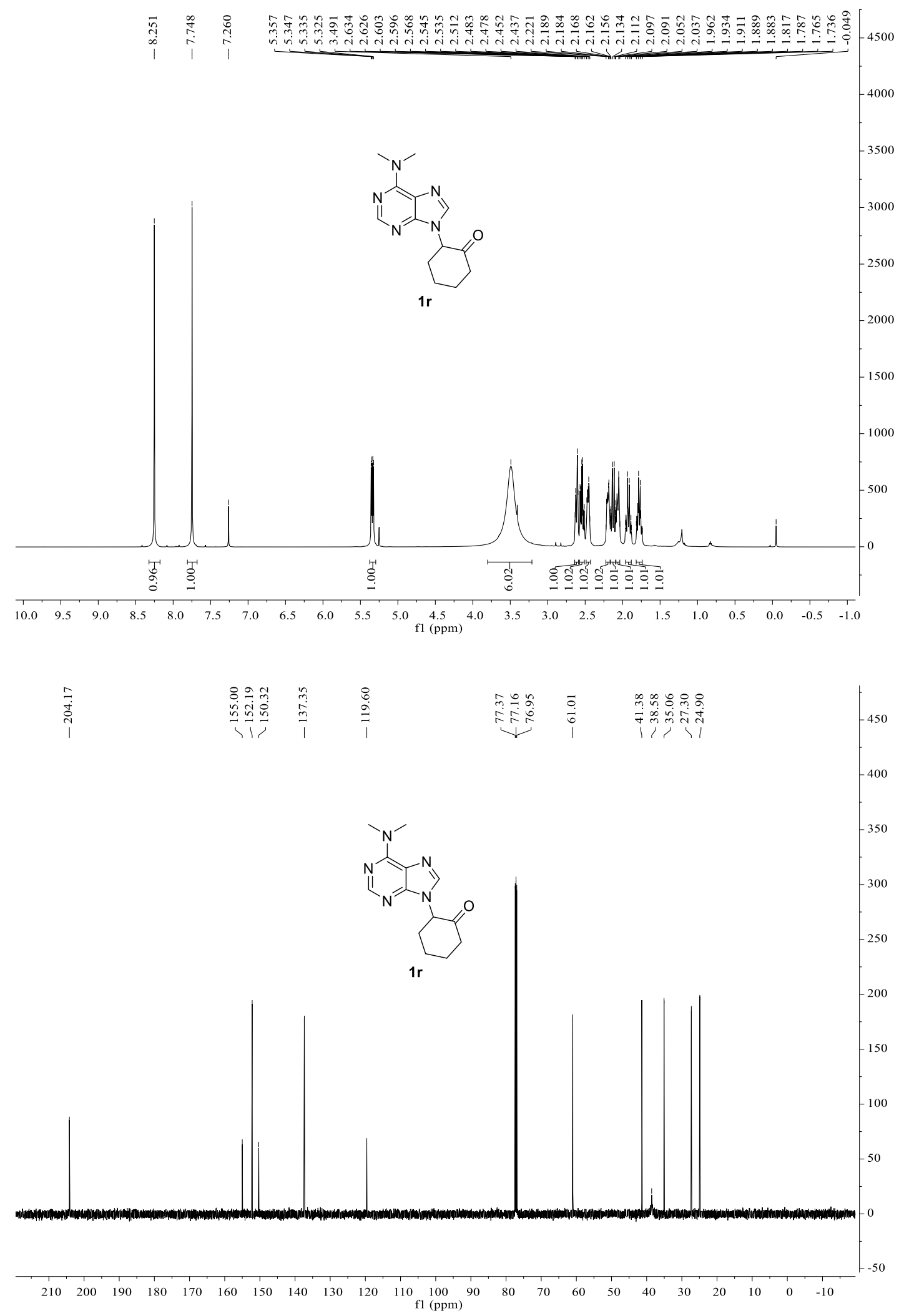




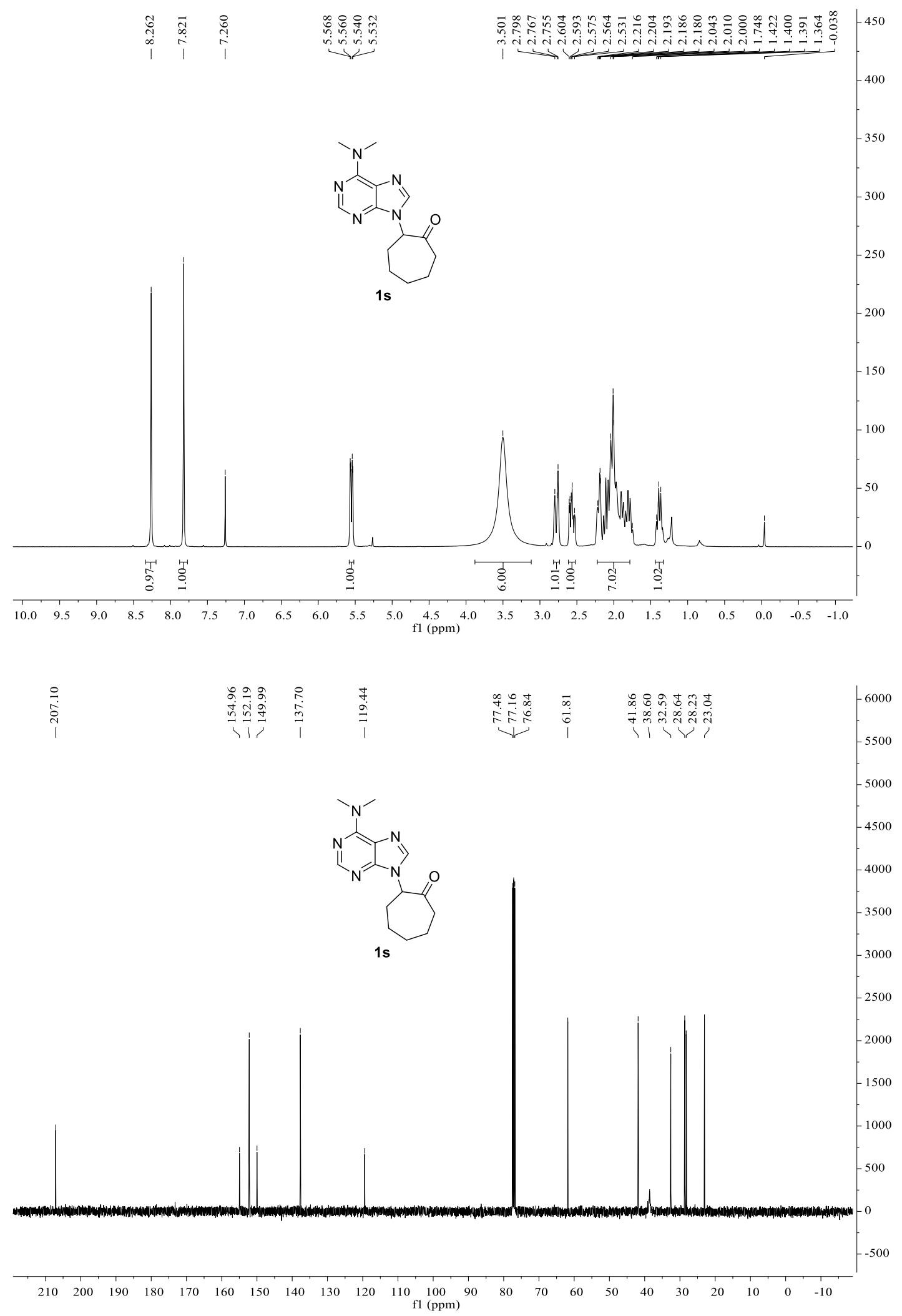




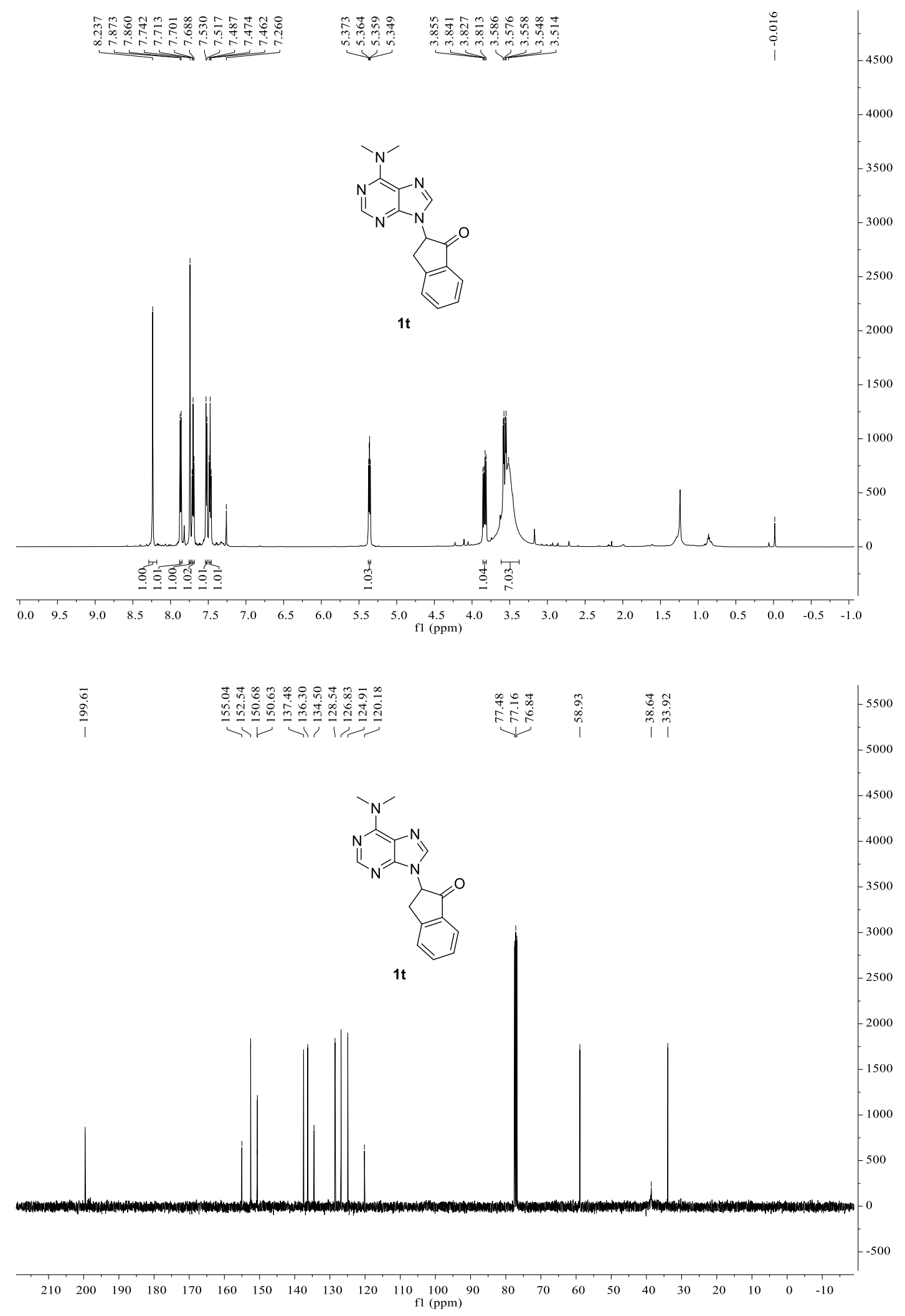




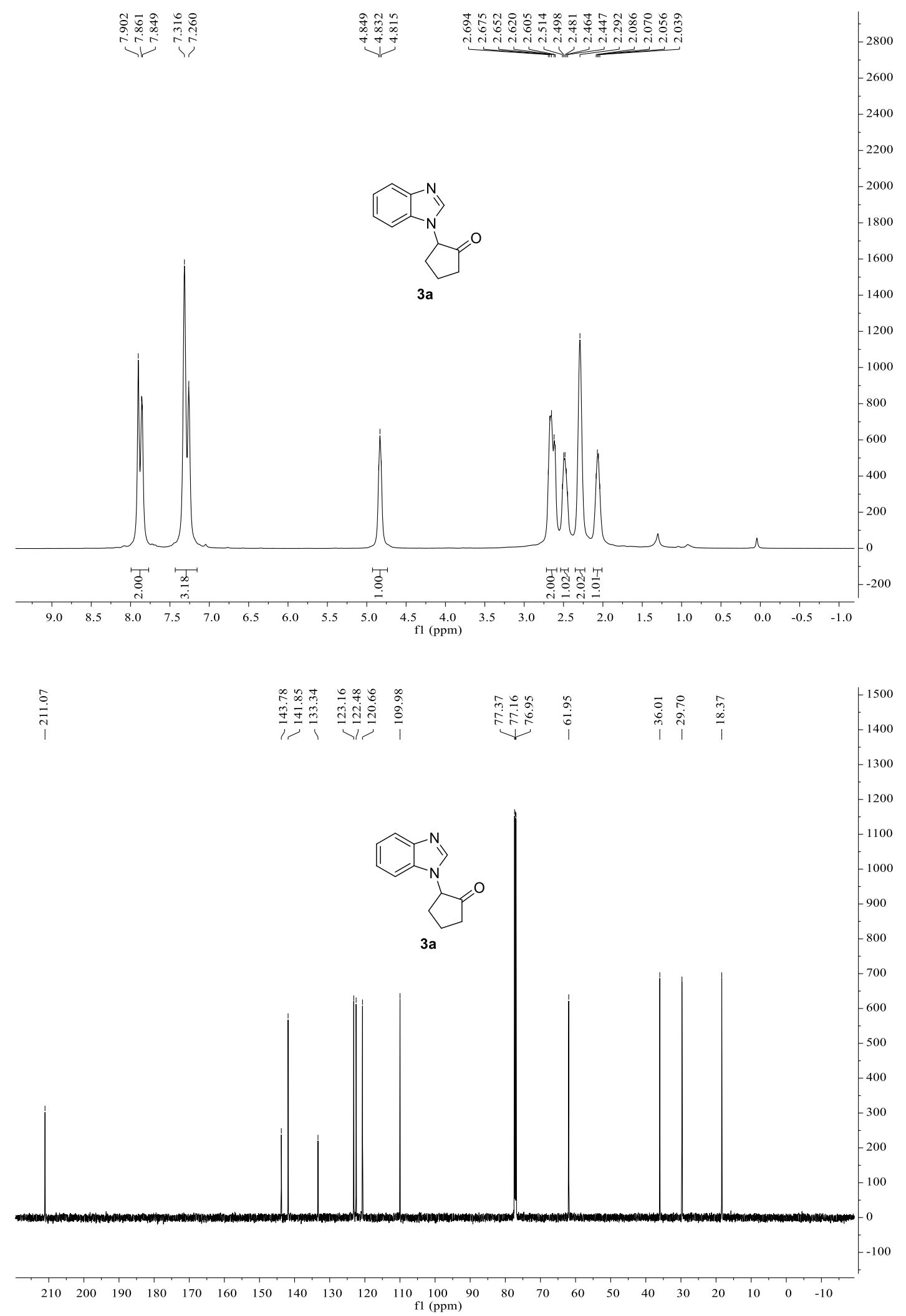




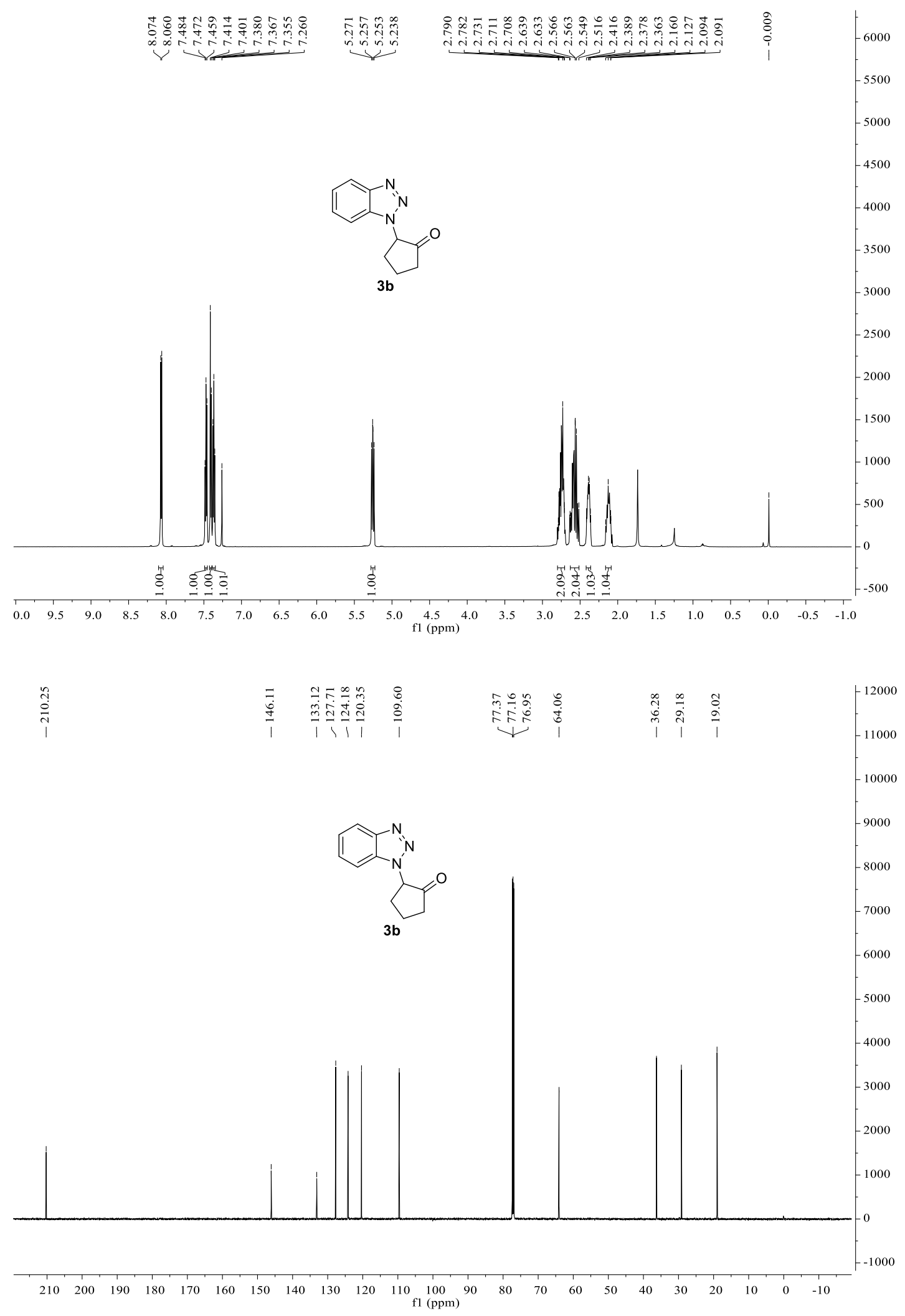




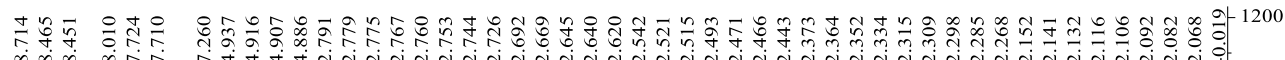

$-1000$

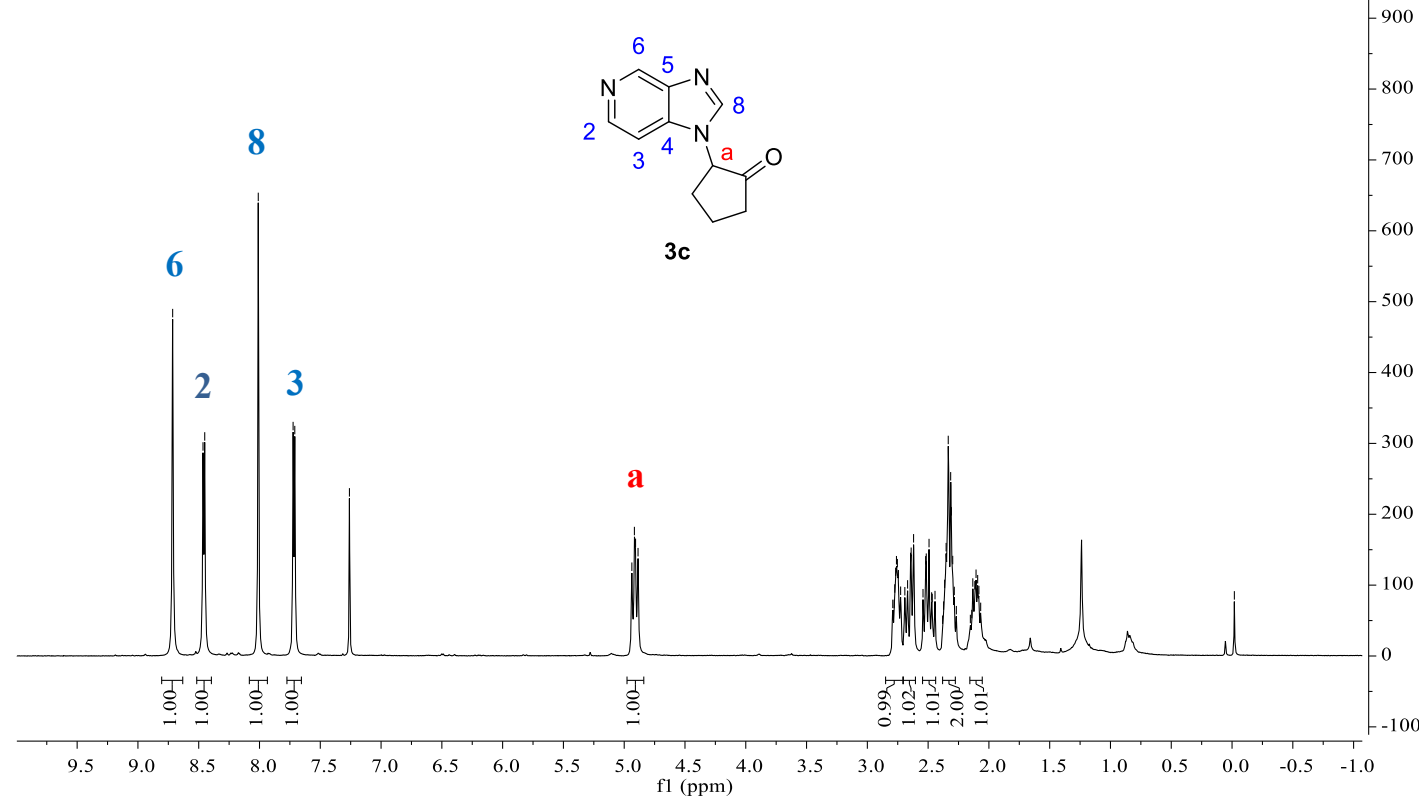

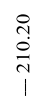

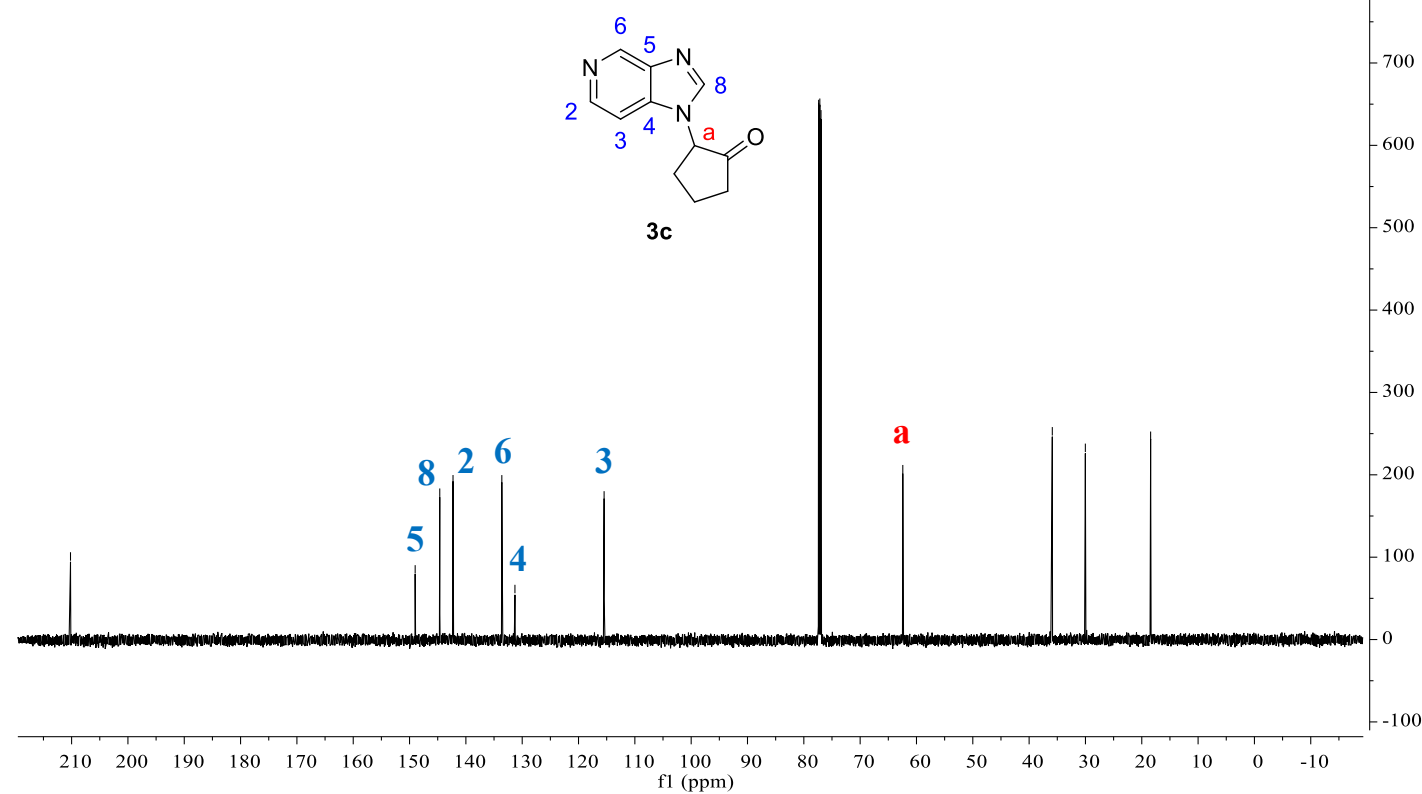




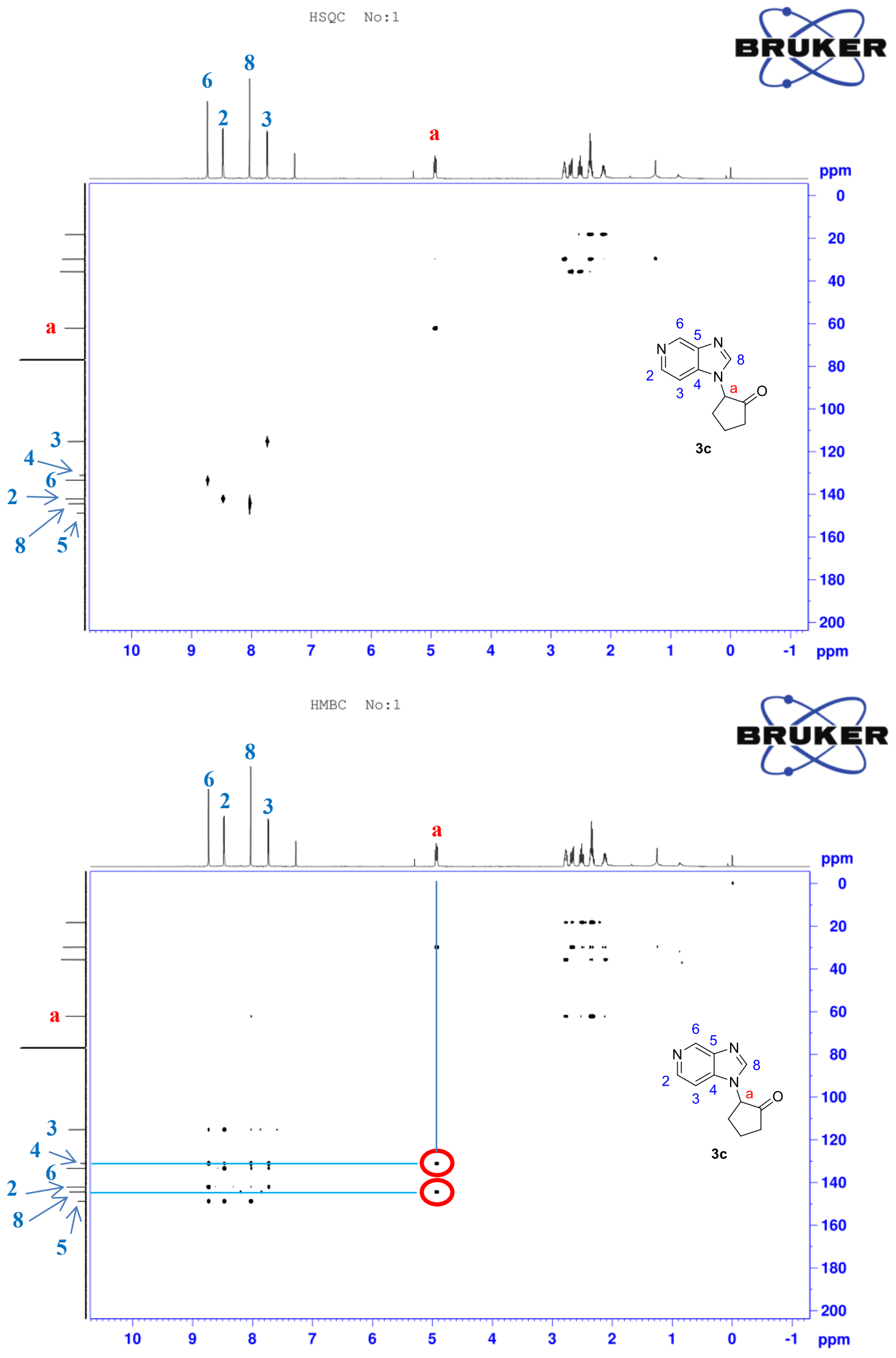




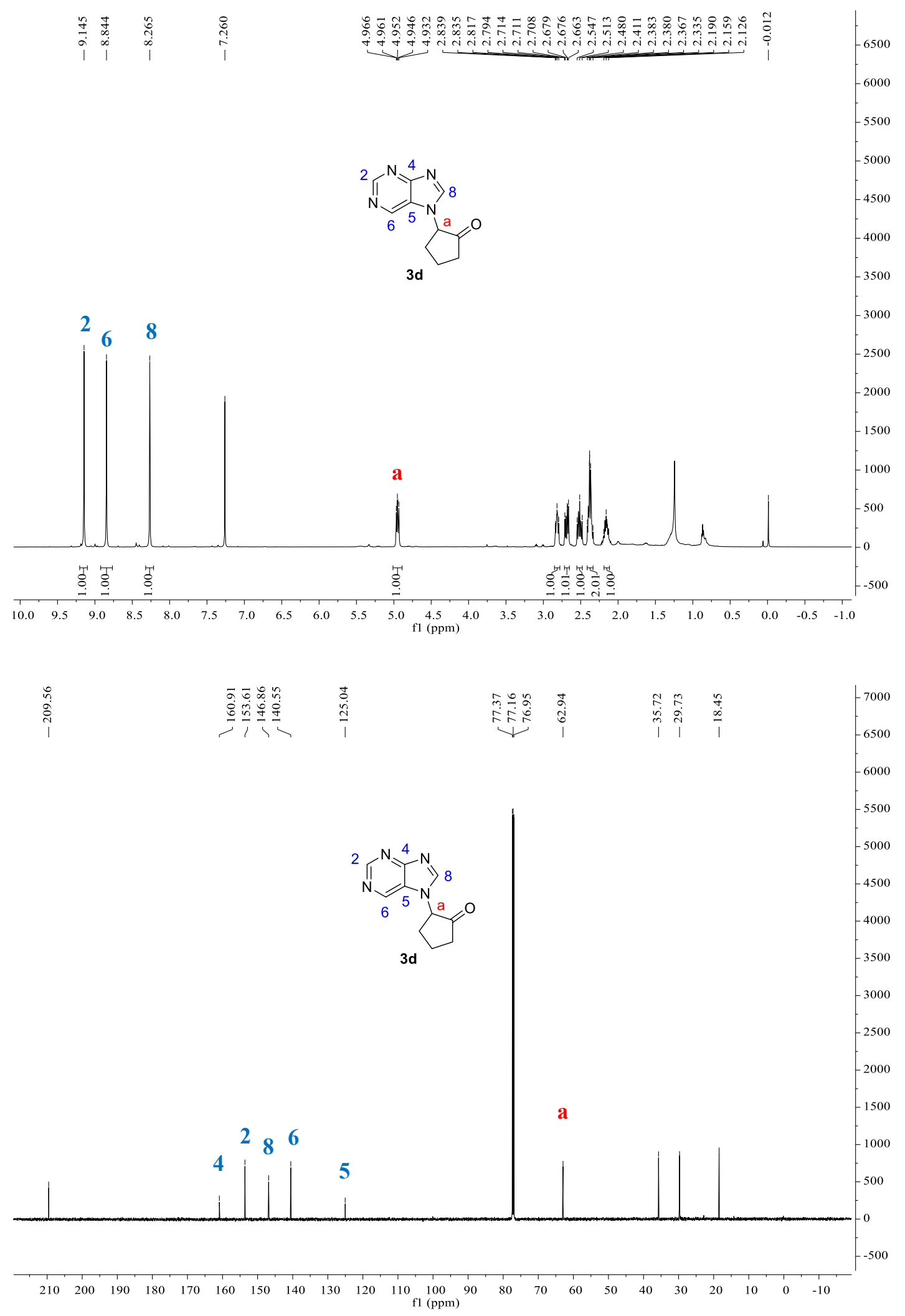



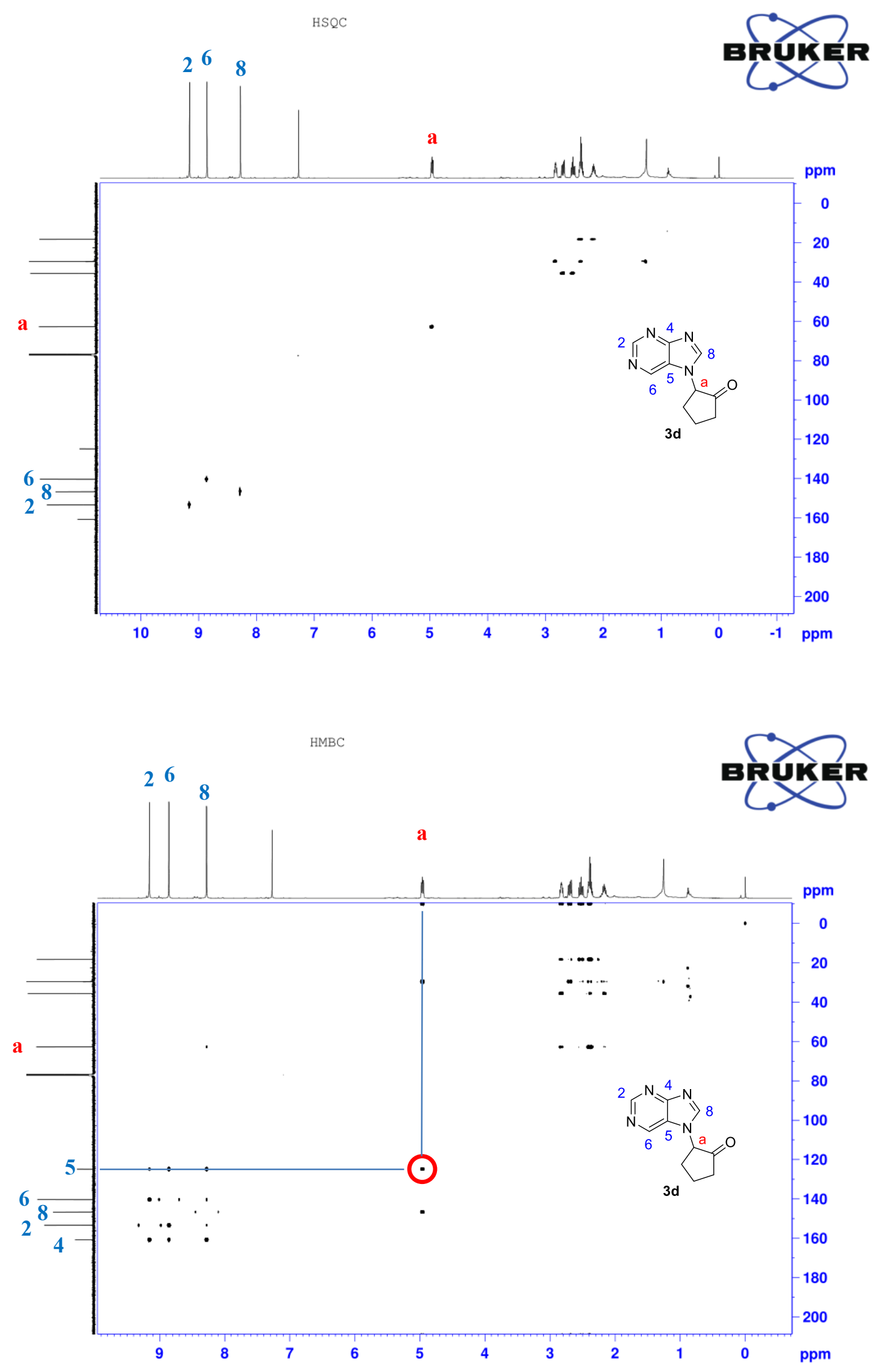

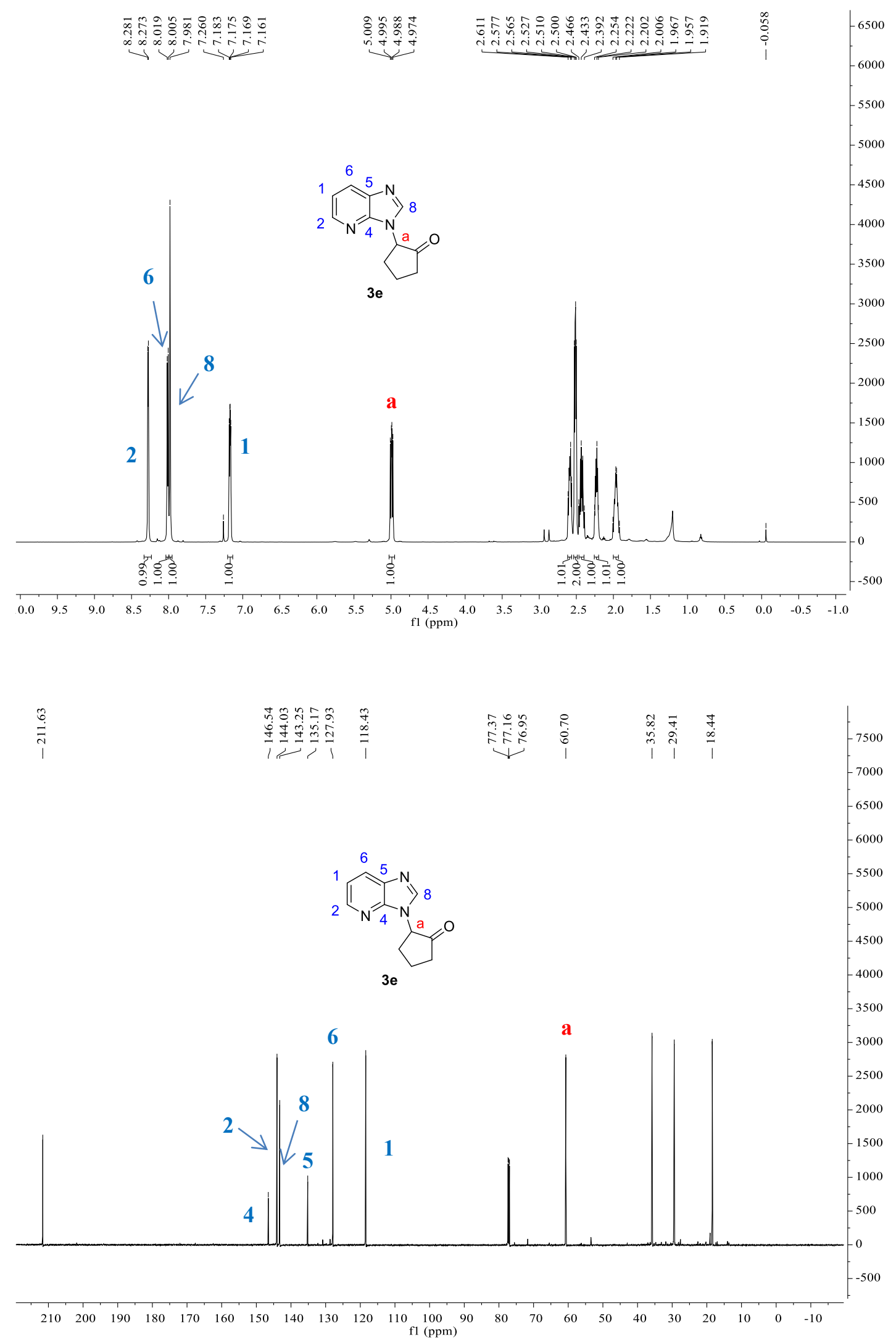

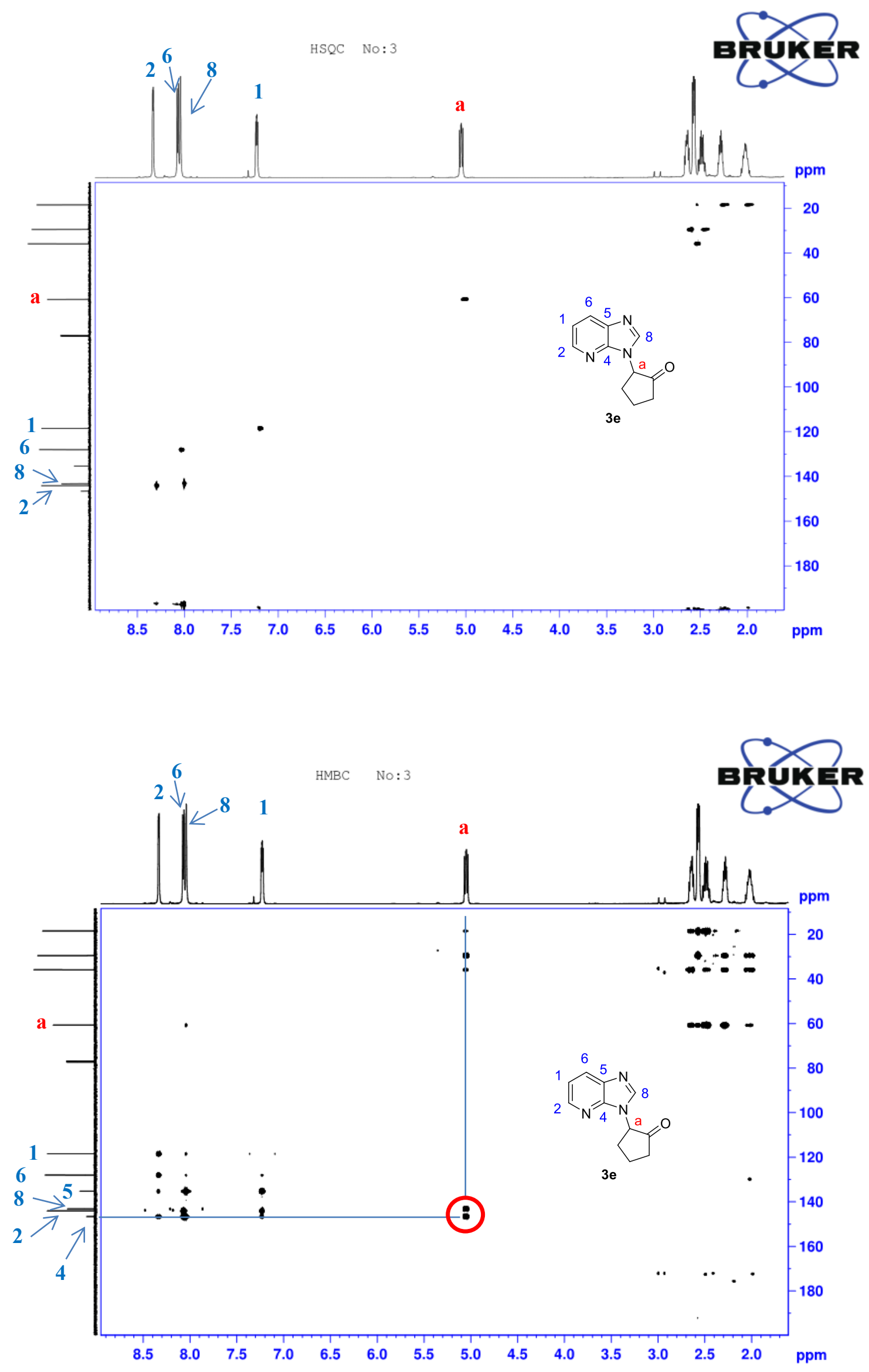


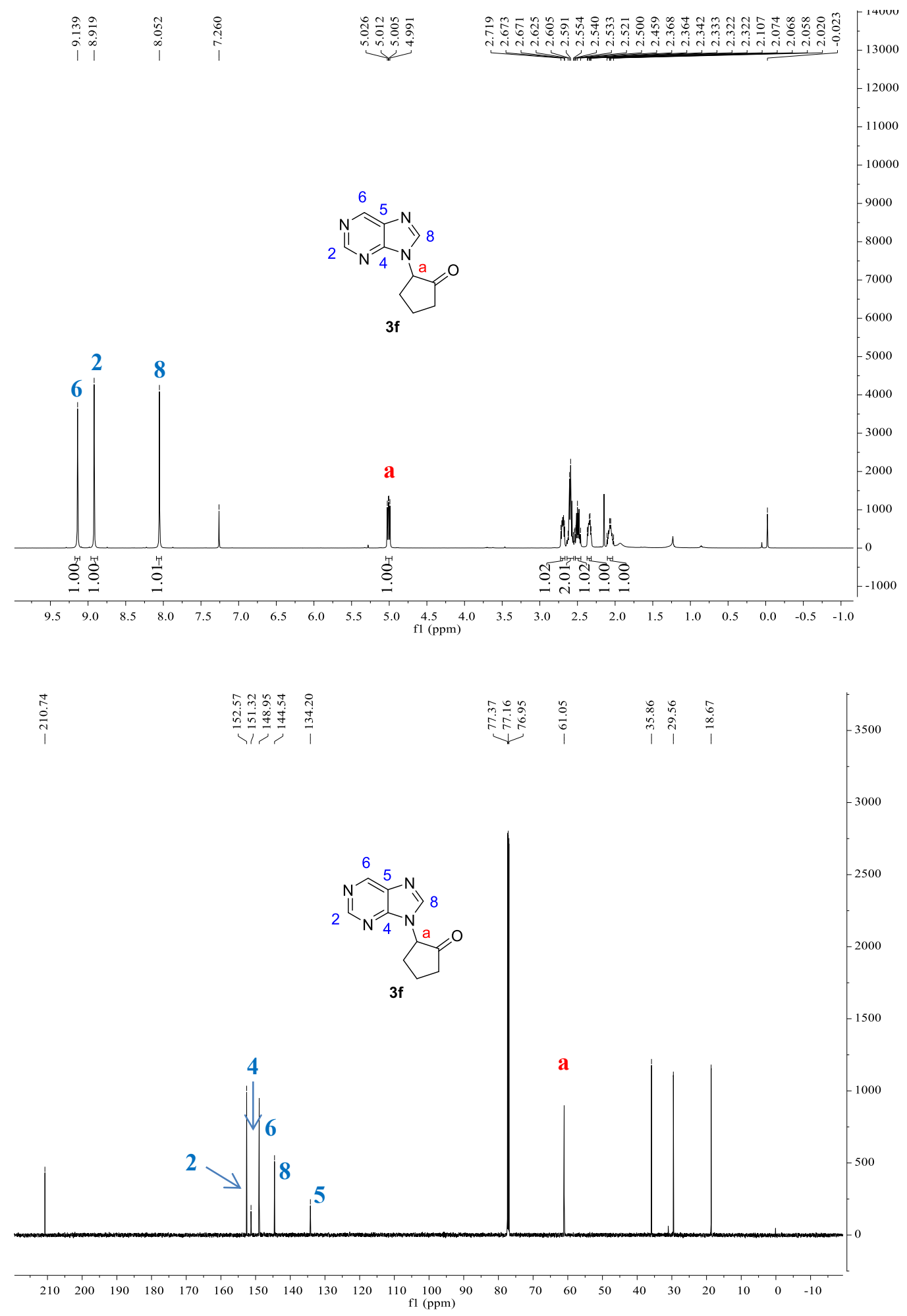



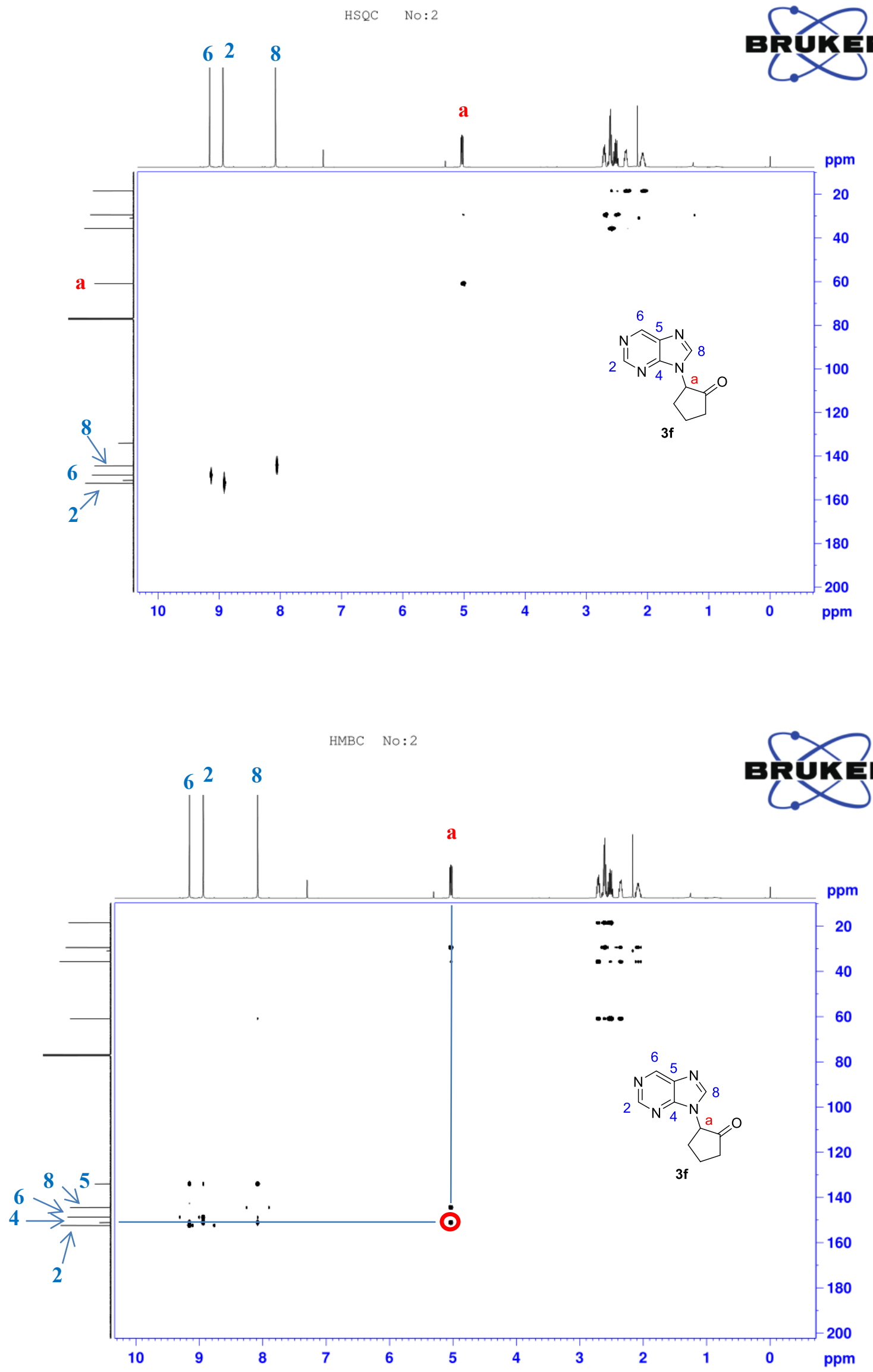


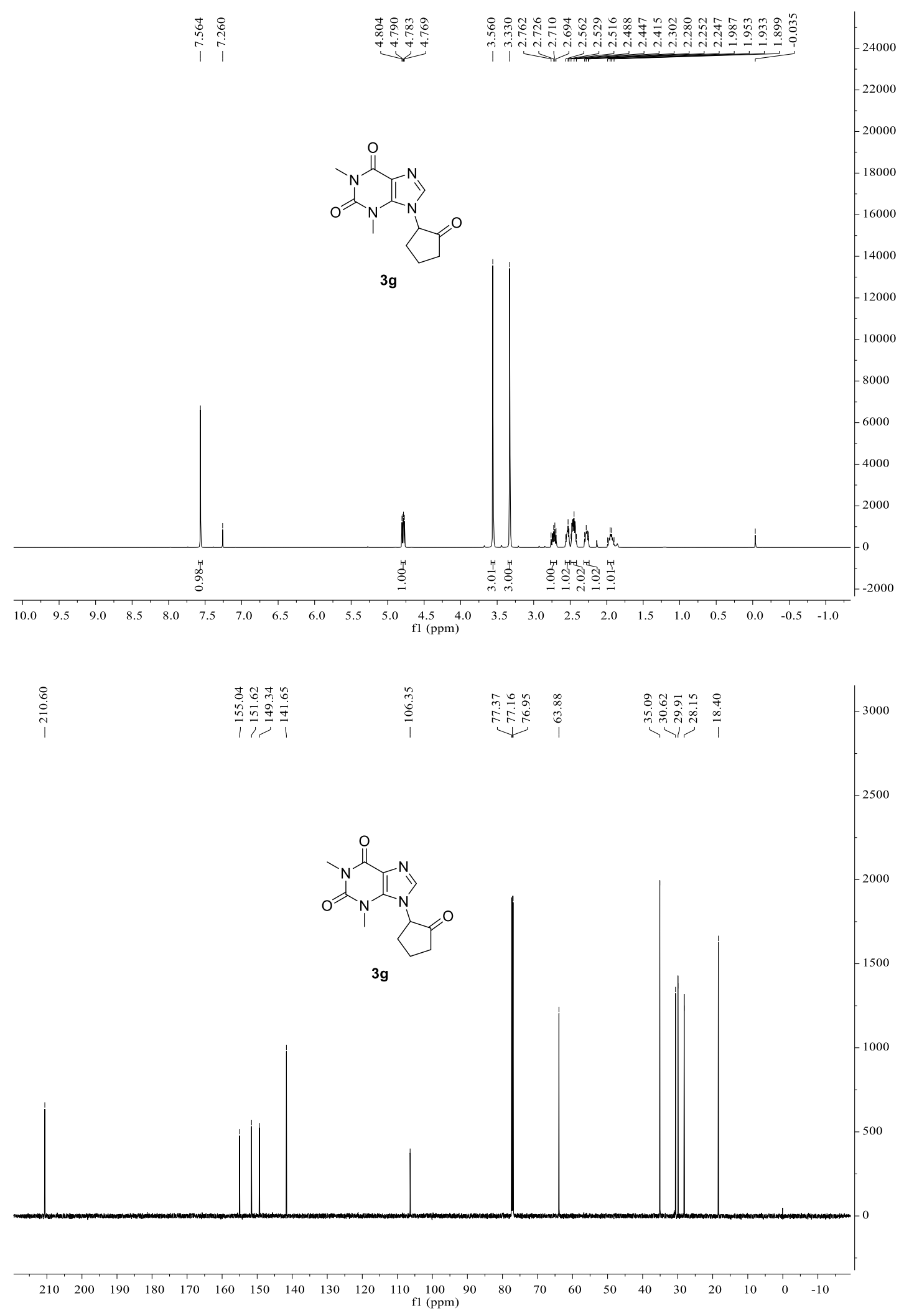




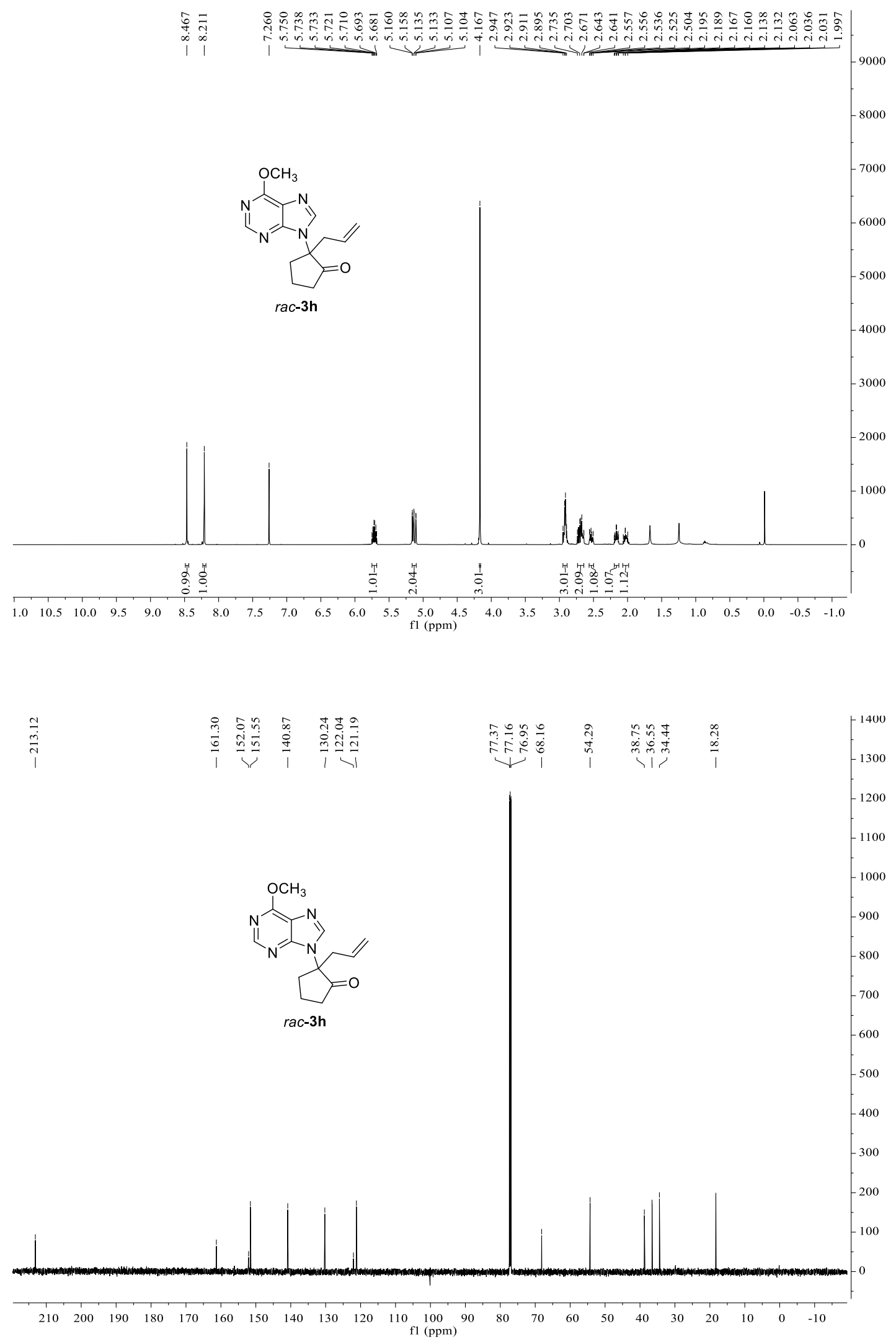



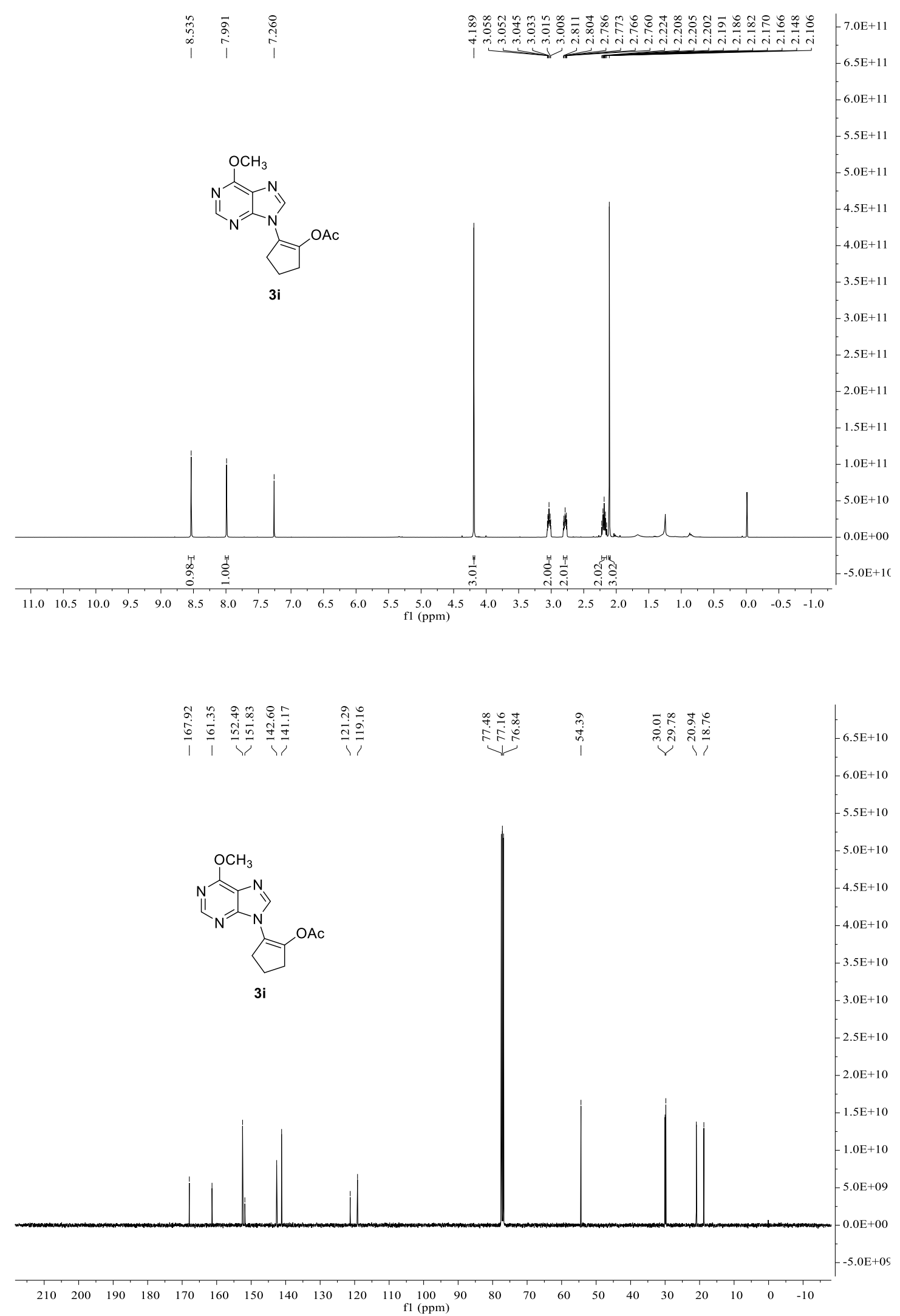


\section{(2) Copies of NMR spectra of products}
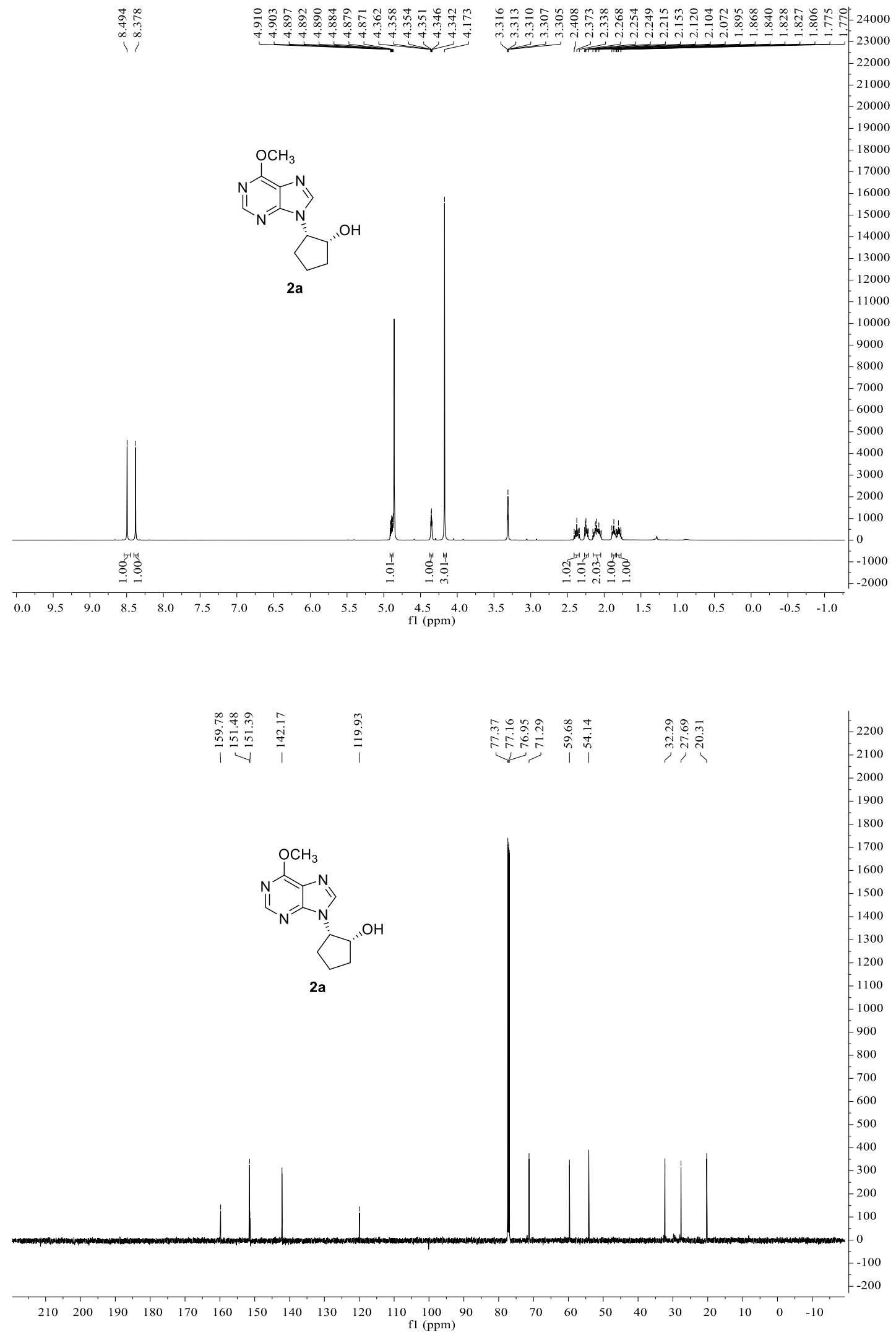


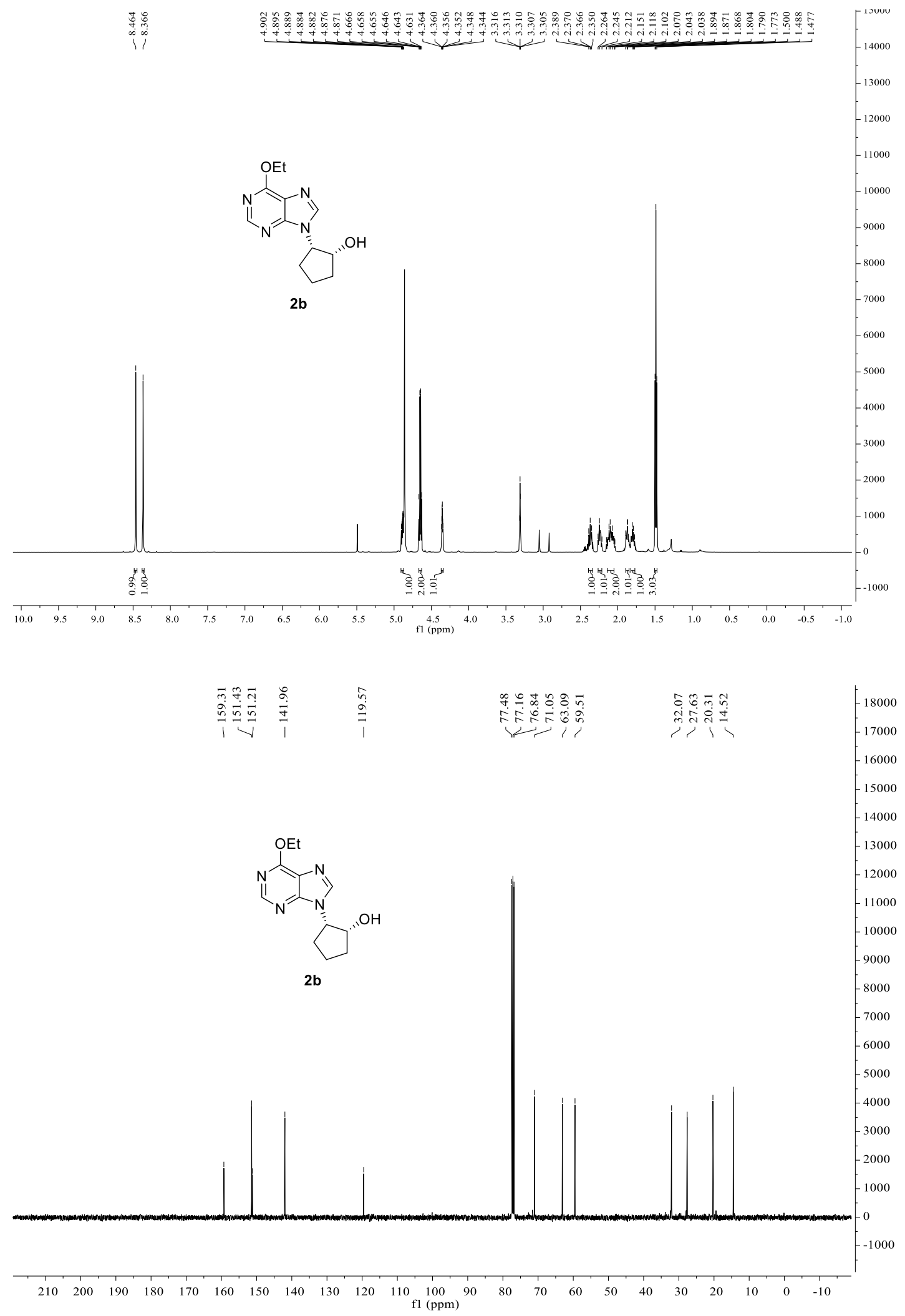




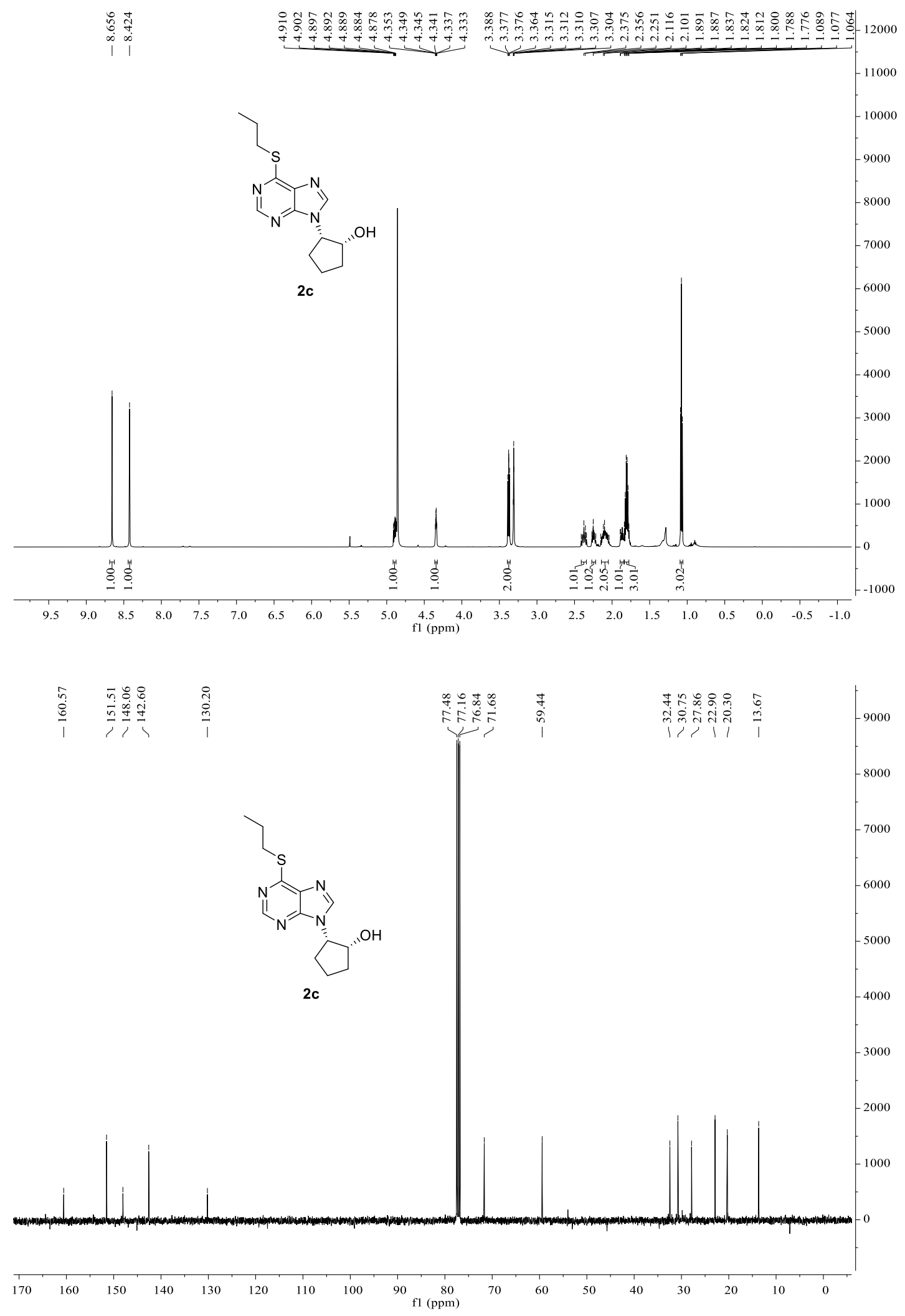




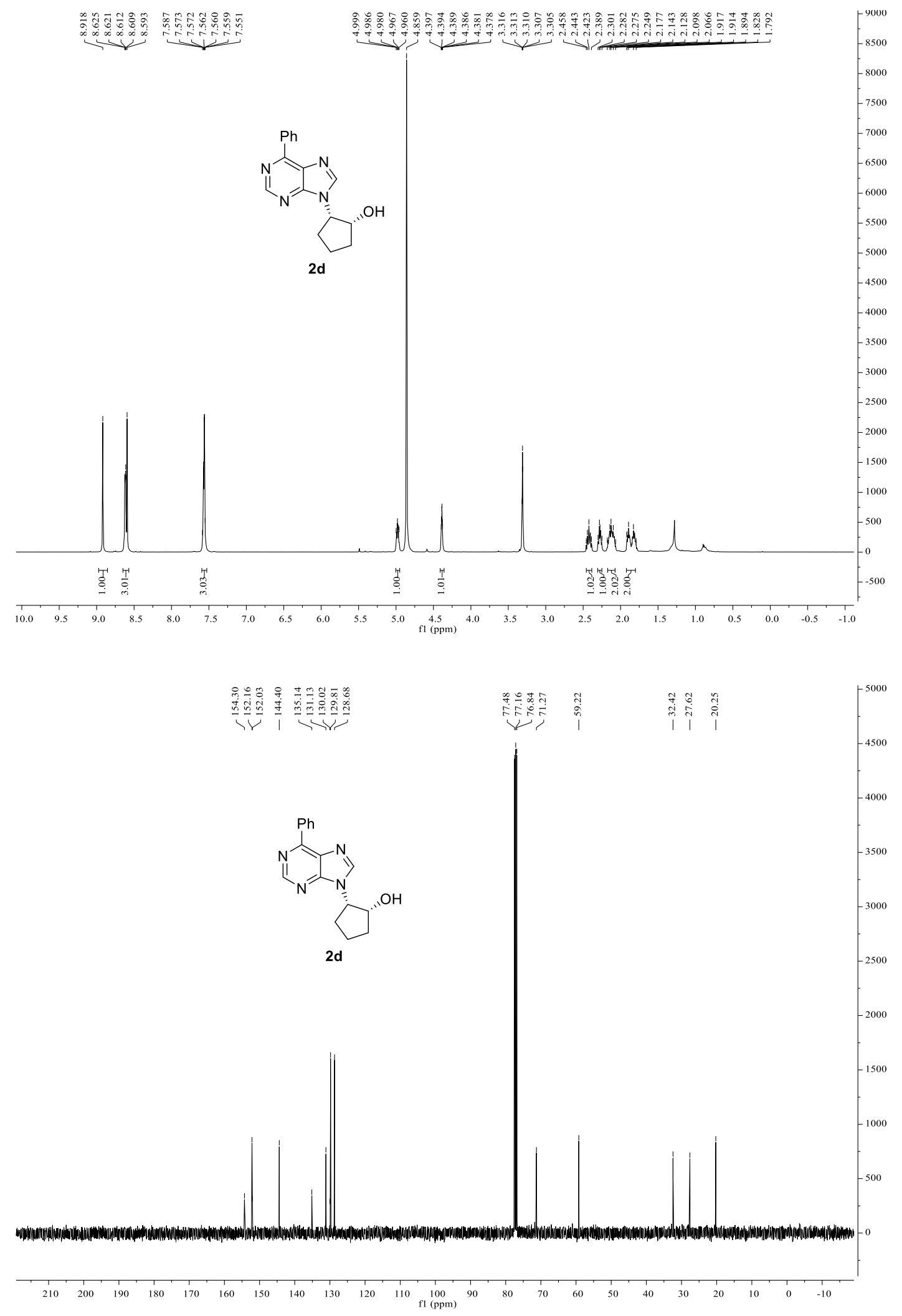




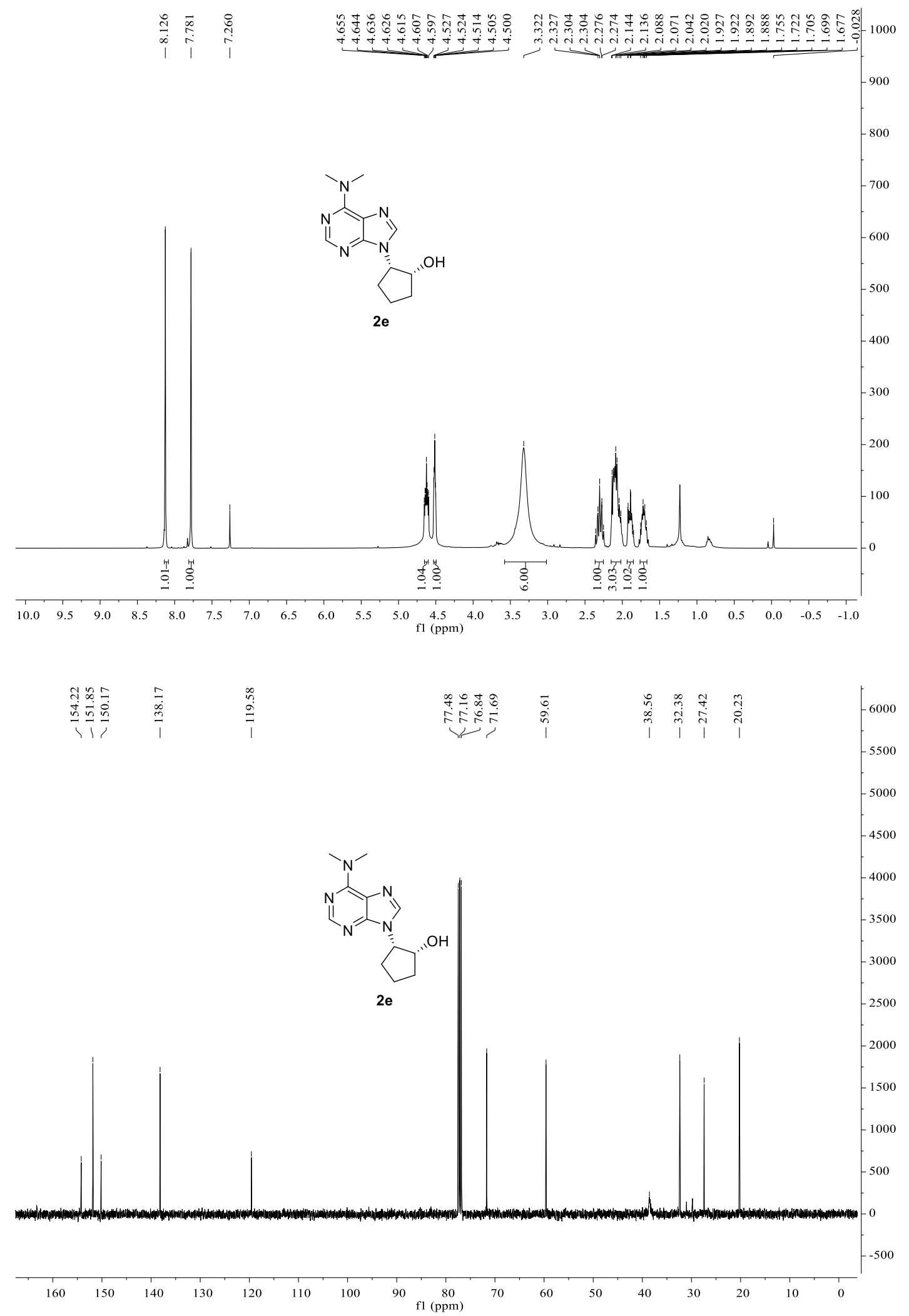



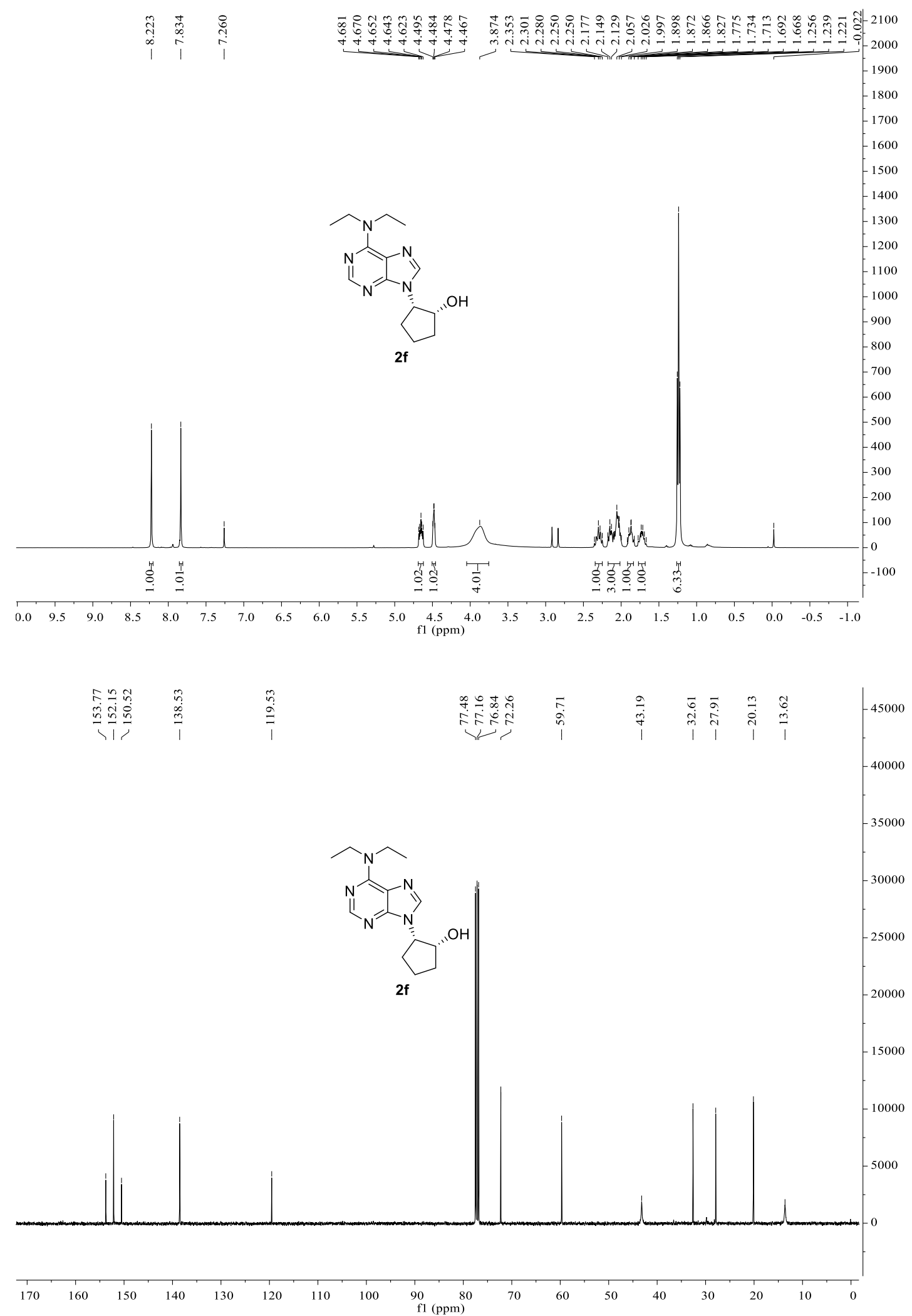


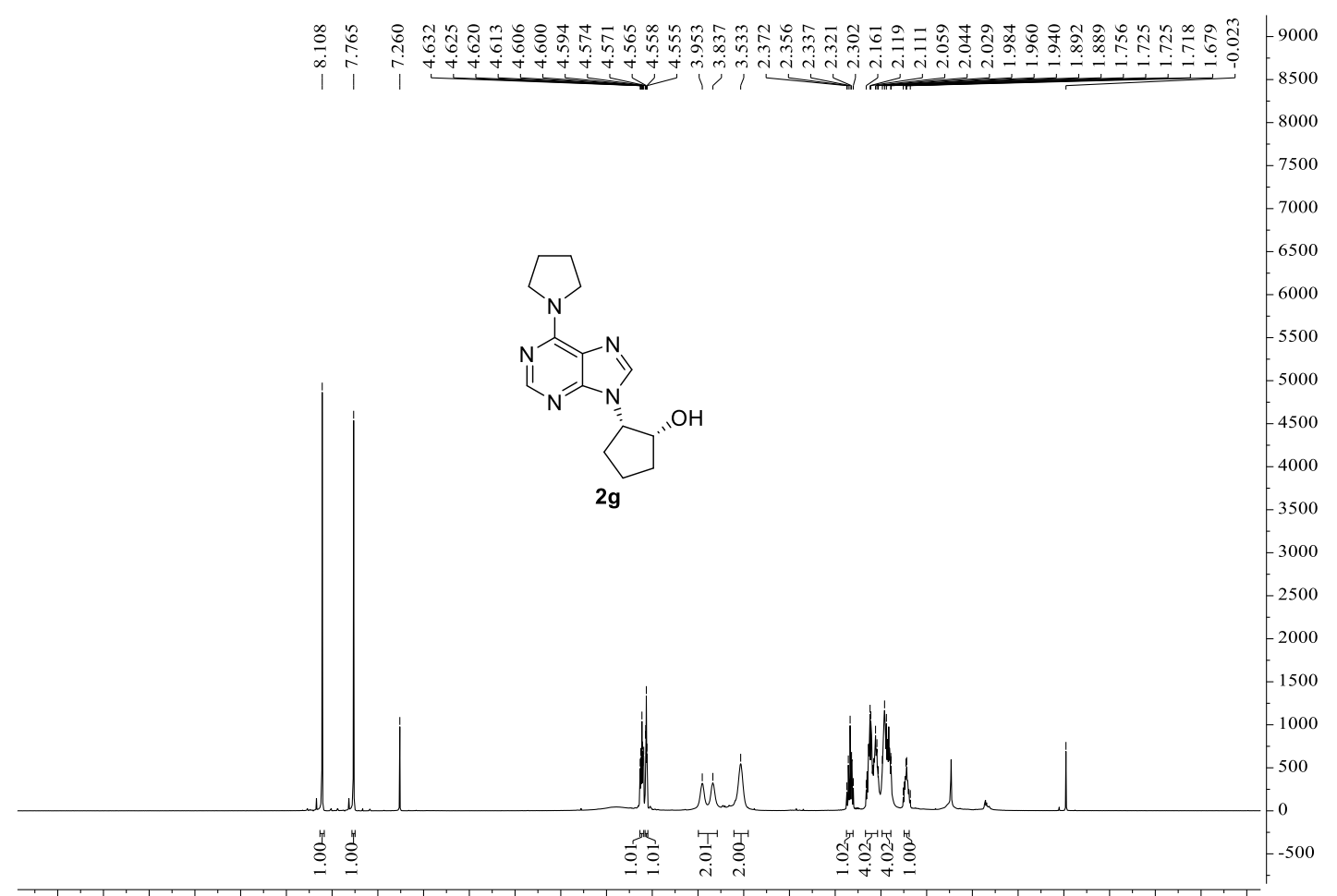

$\begin{array}{rlllllllllllllllllllllllllllll}11.0 & 10.5 & 10.0 & 9.5 & 9.0 & 8.5 & 8.0 & 7.5 & 7.0 & 6.5 & 6.0 & 5.5 & 5.0 & 4.5 & 4.0 & 3.5 & 3.0 & 2.5 & 2.0 & 1.5 & 1.0 & 0.5 & 0.0 & -0.5 & -1.0 & -1.5 & -2.0\end{array}$

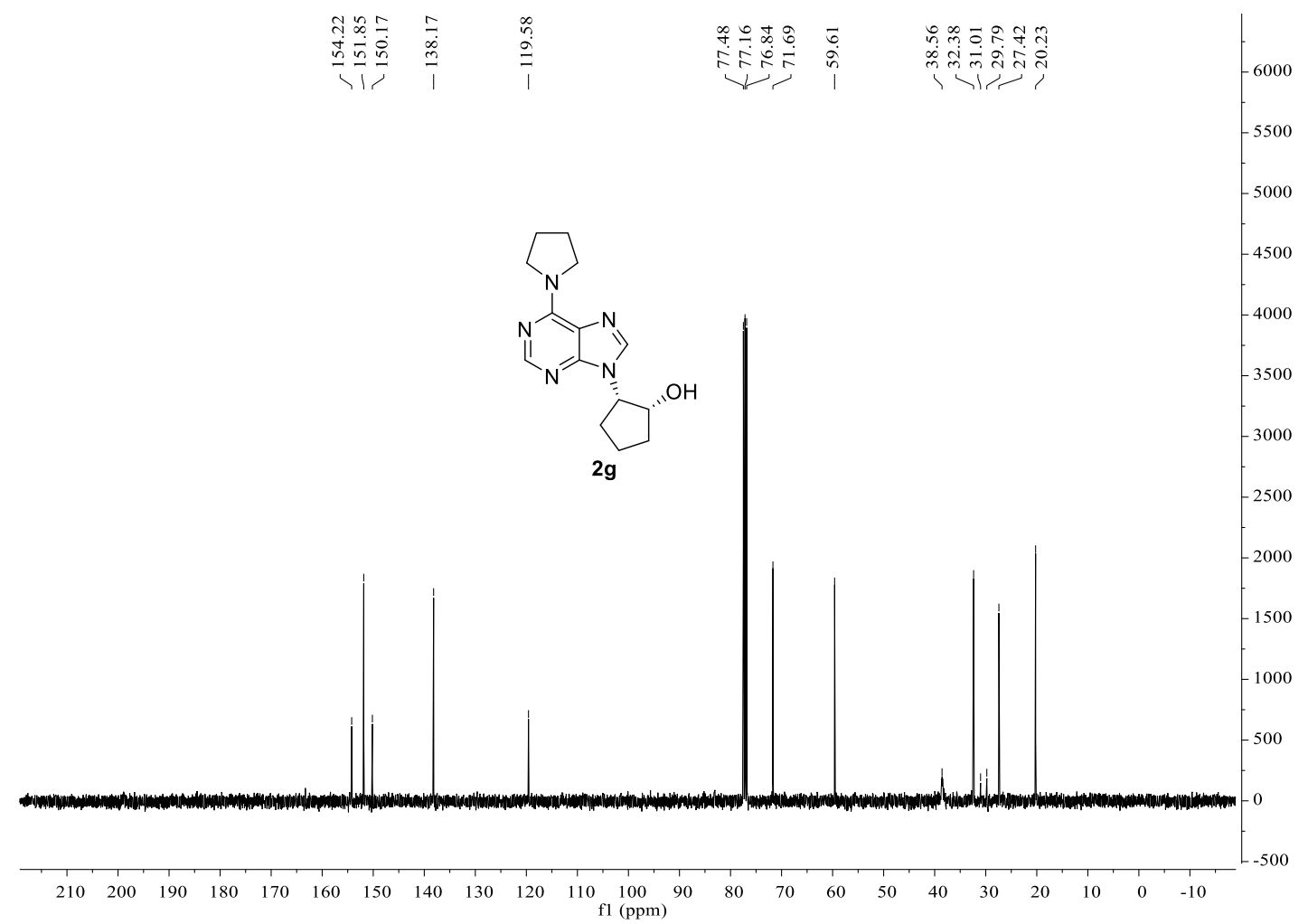




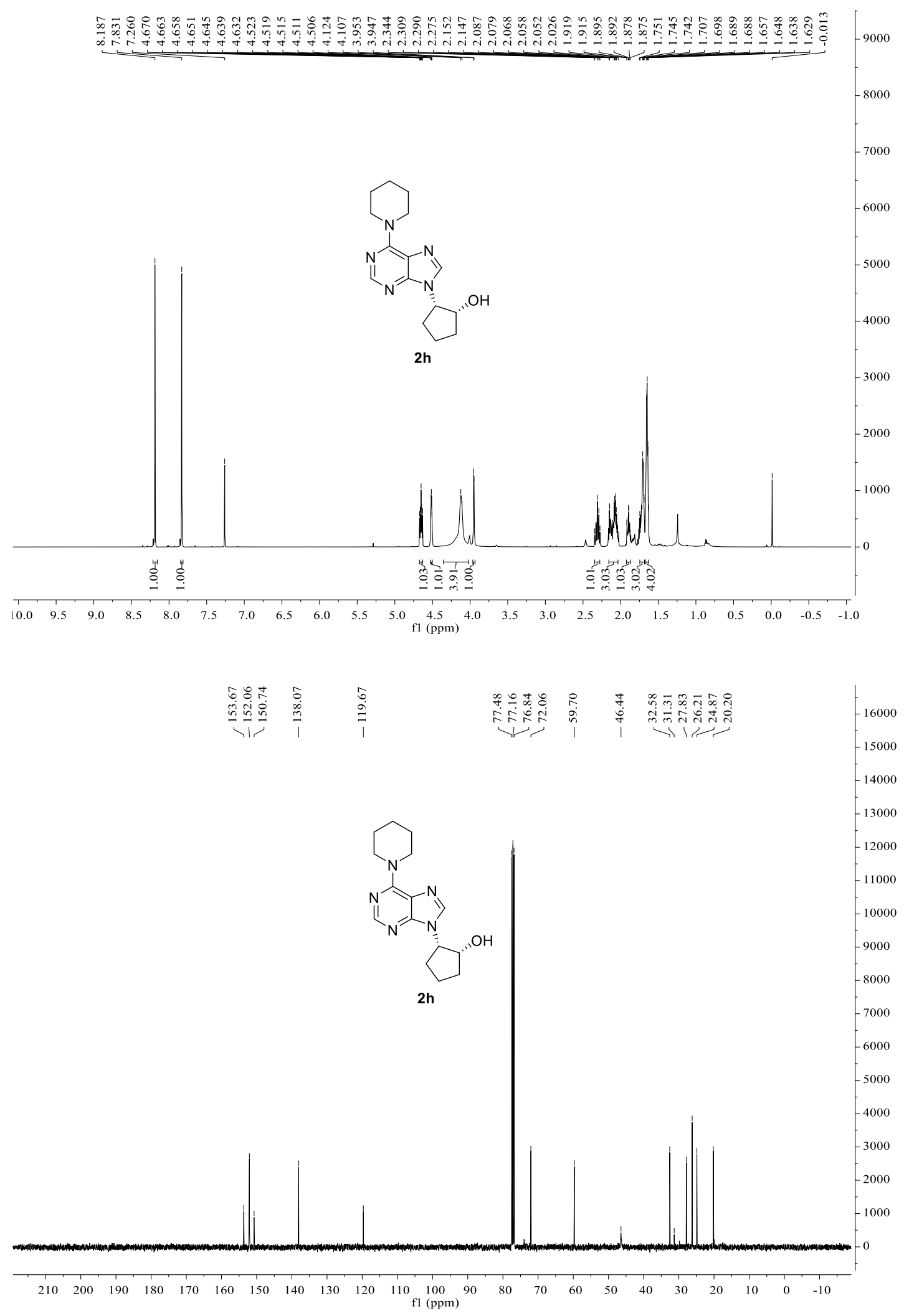




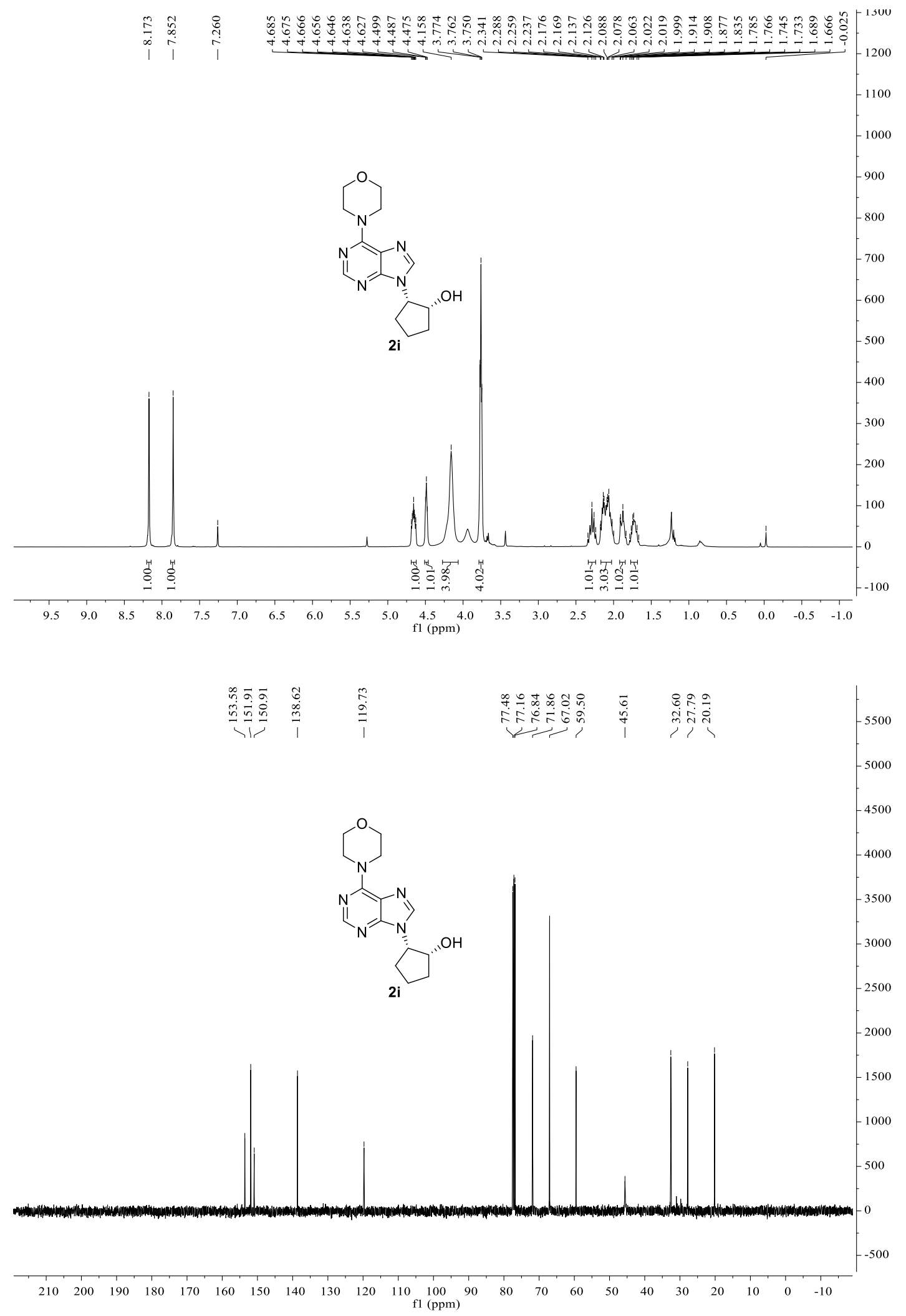



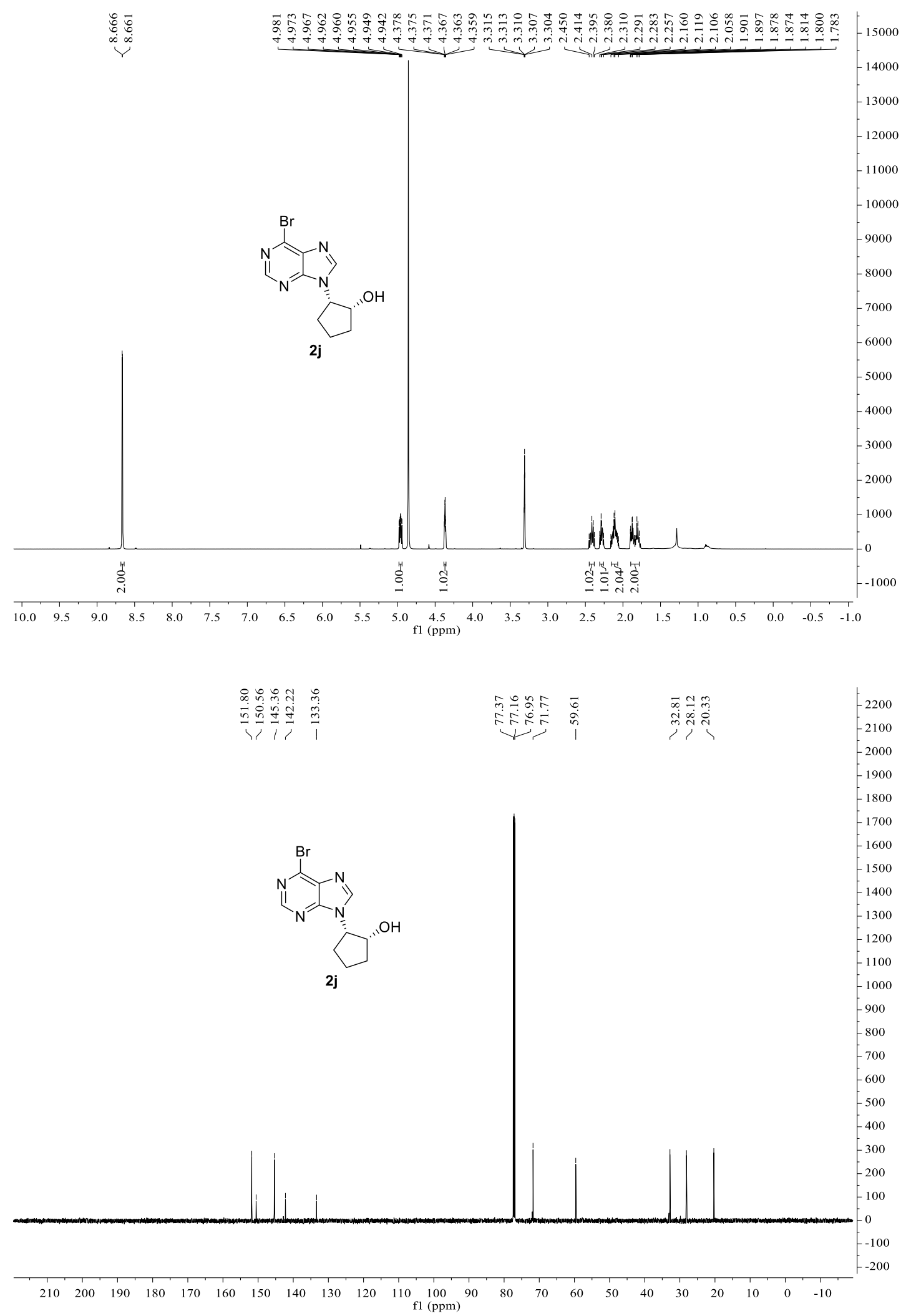

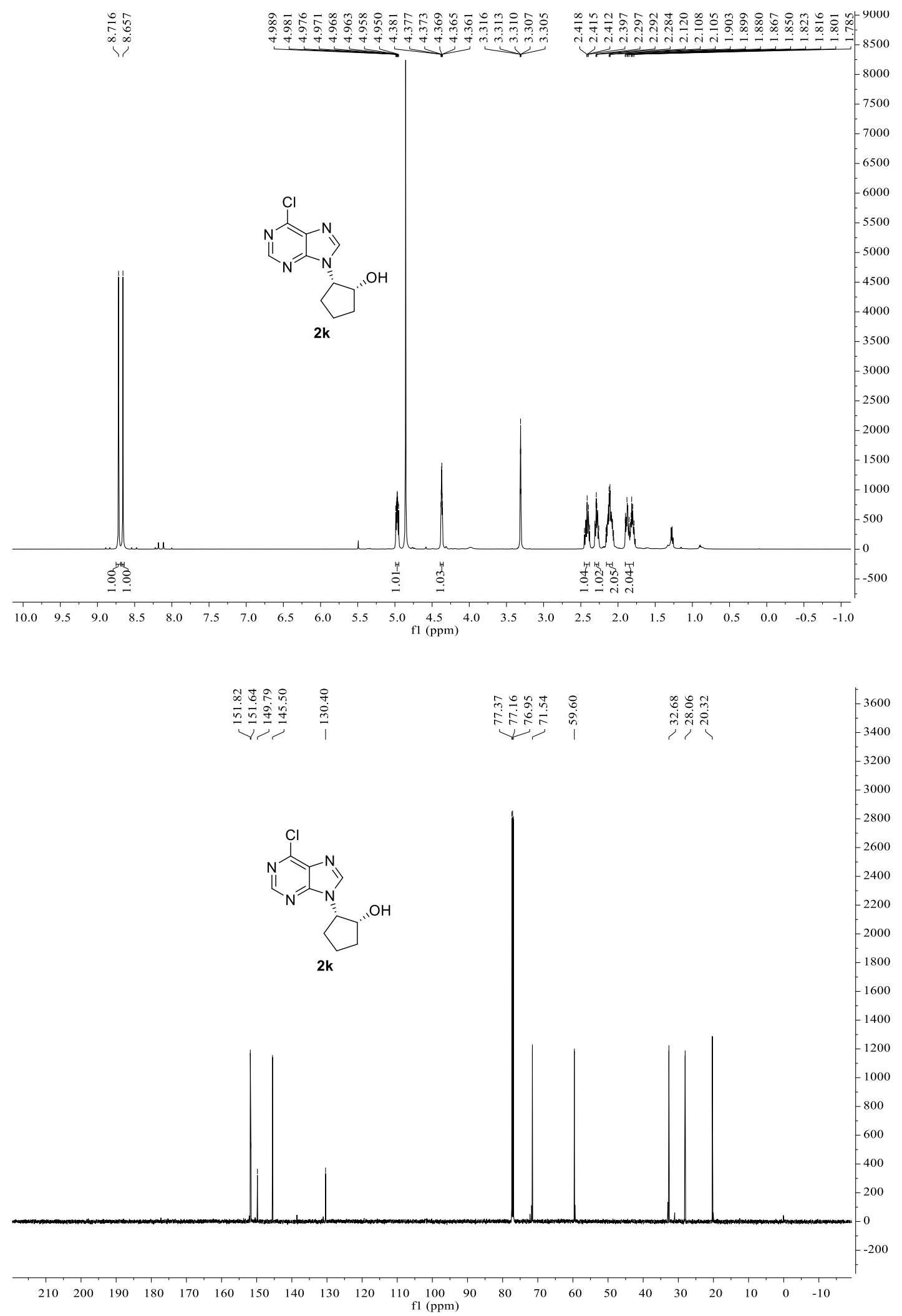


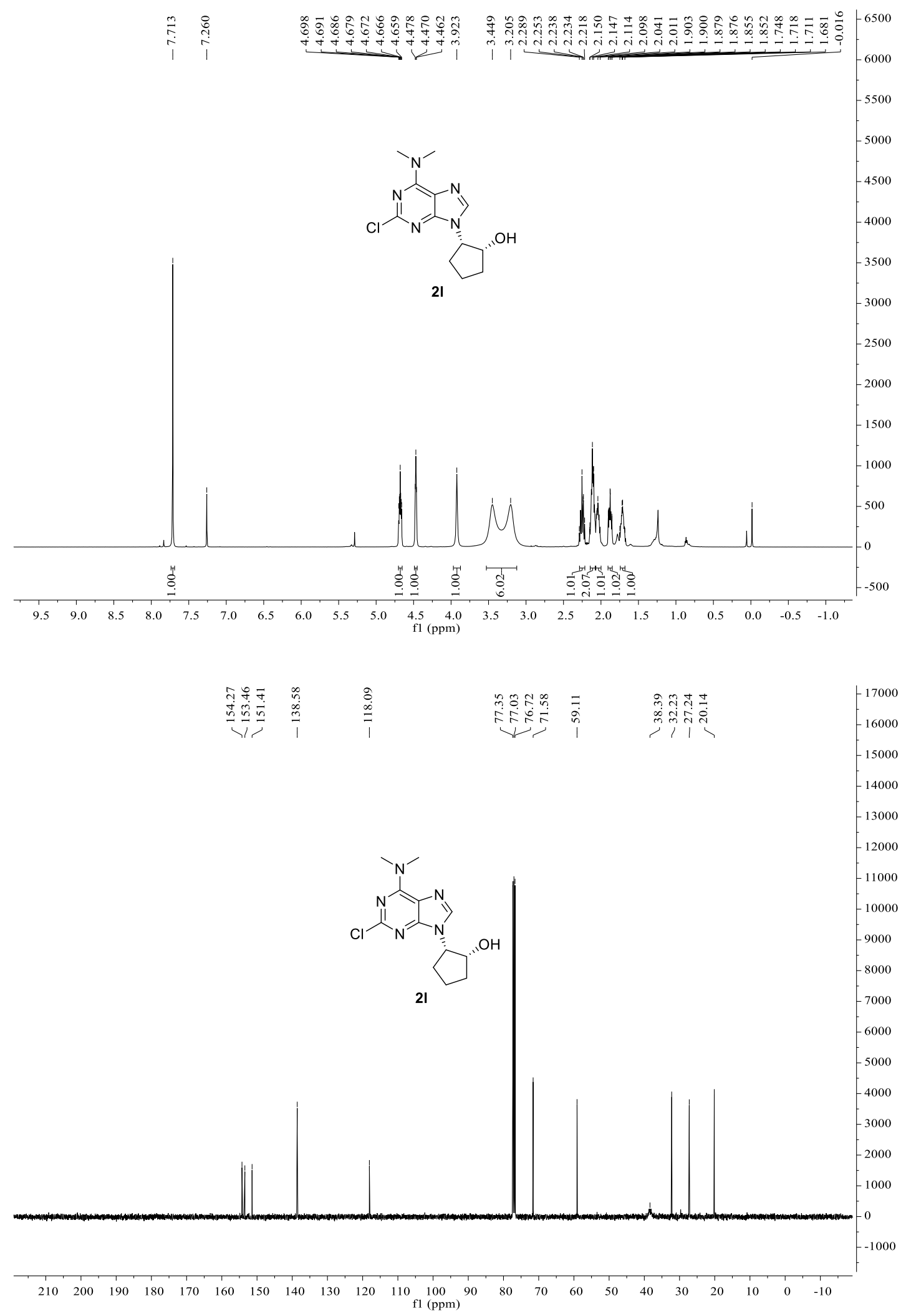



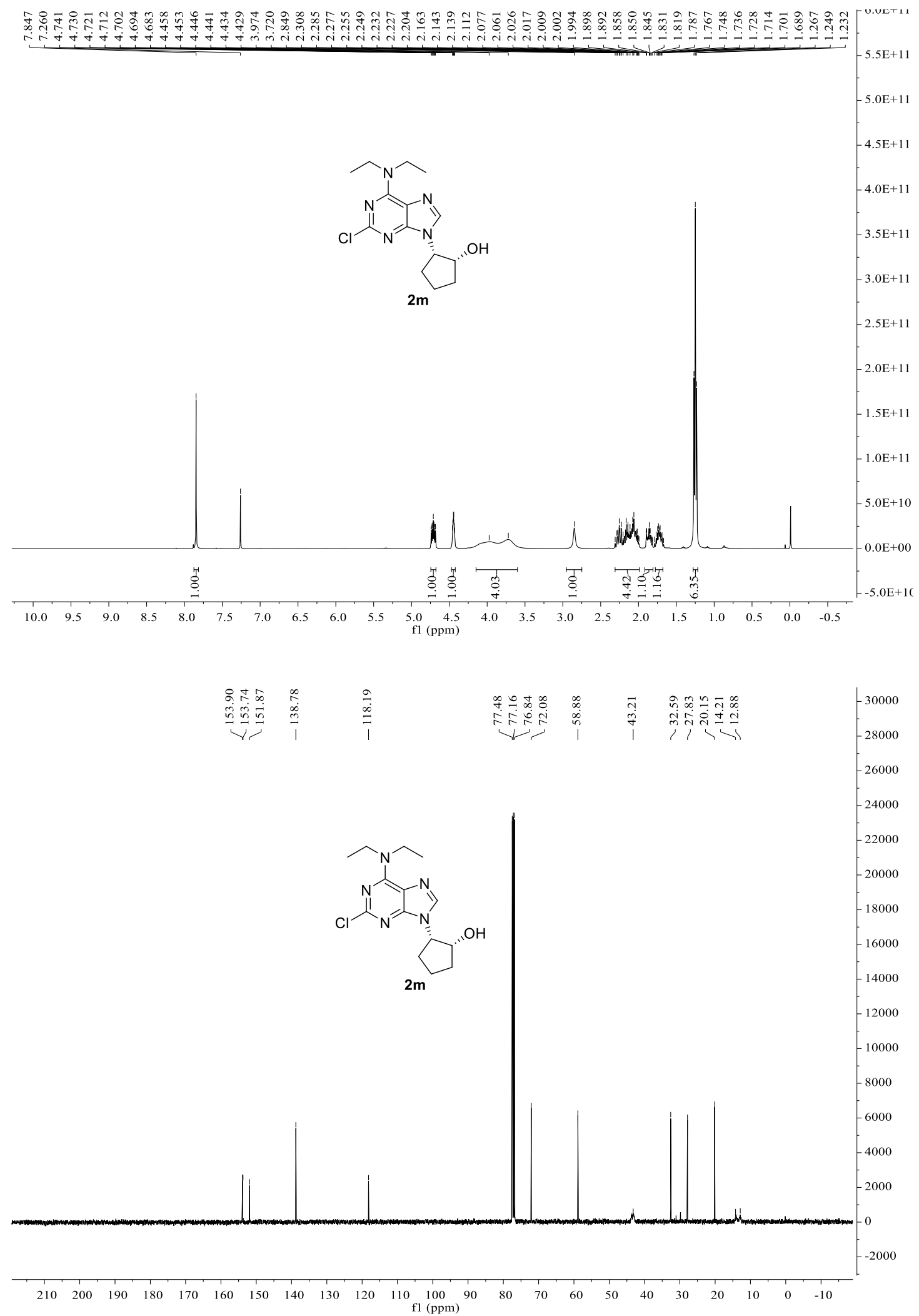


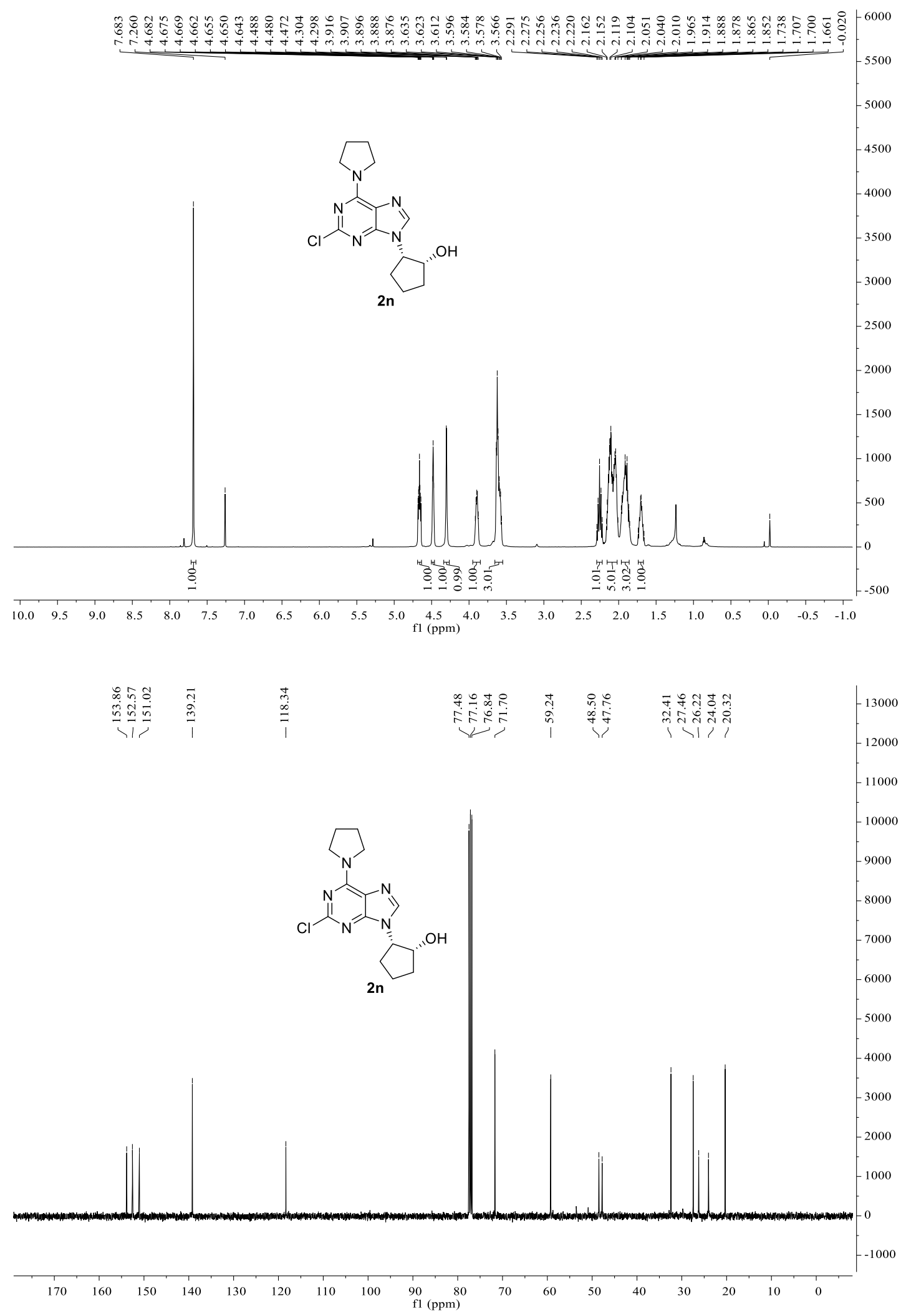




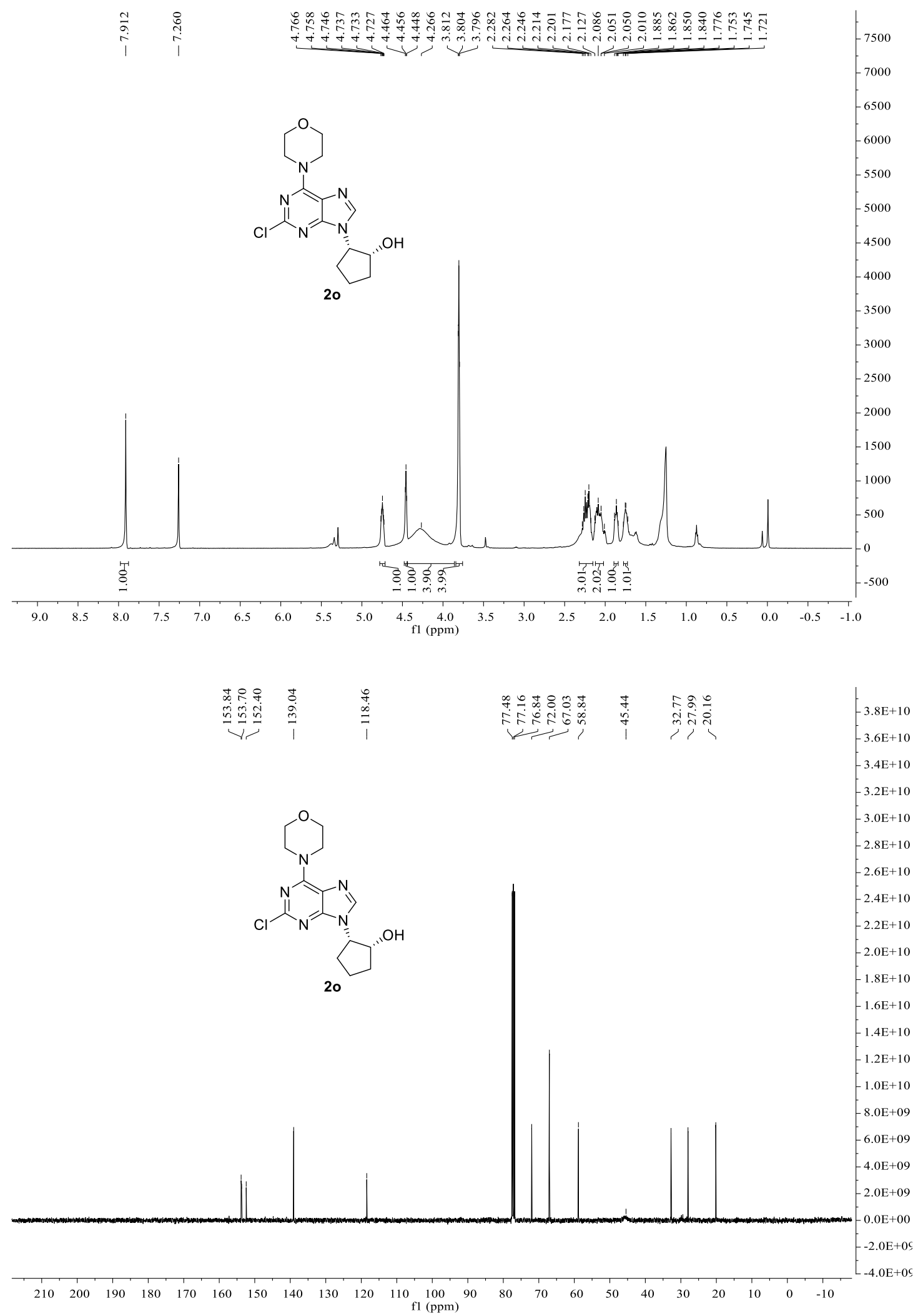



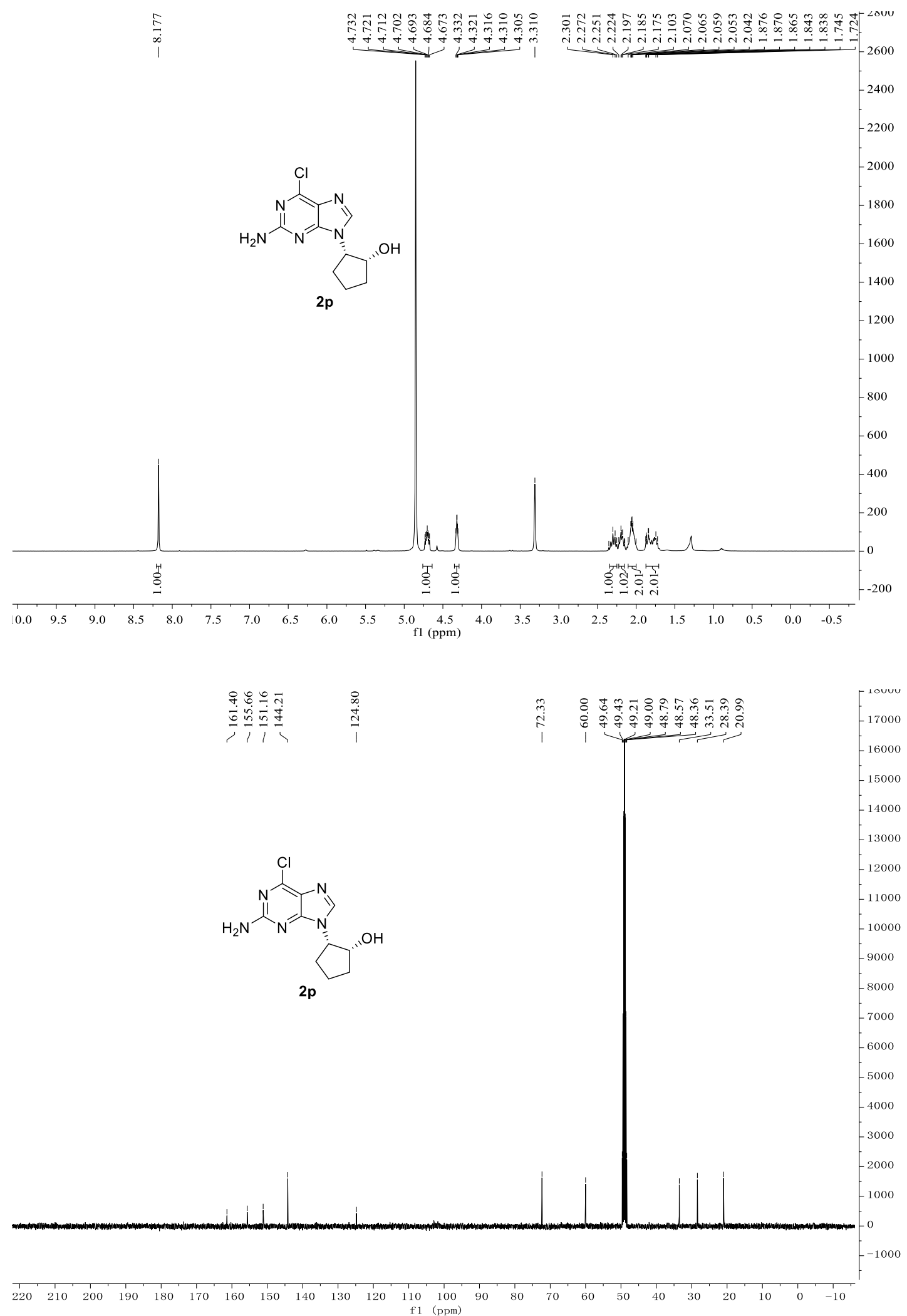


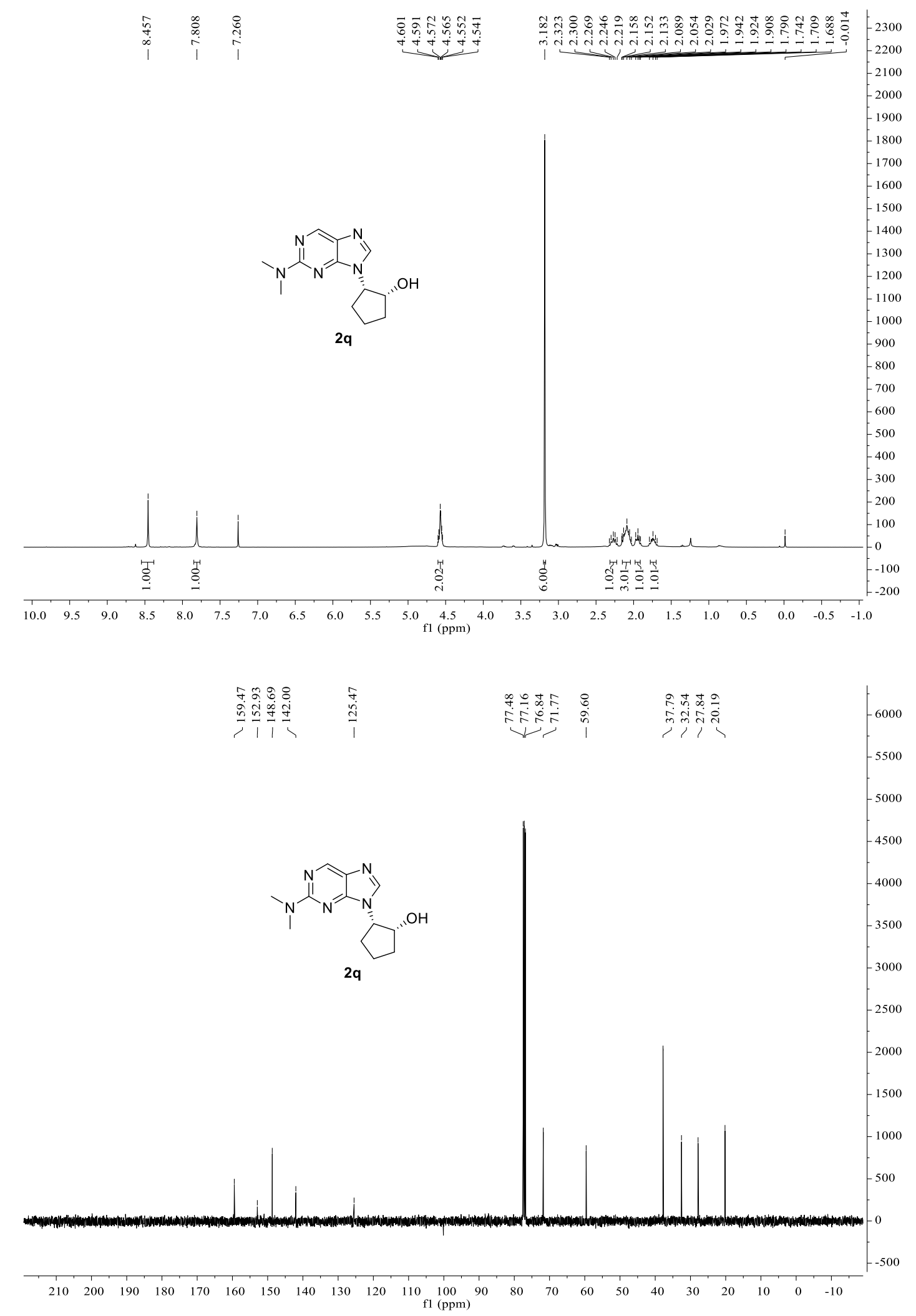



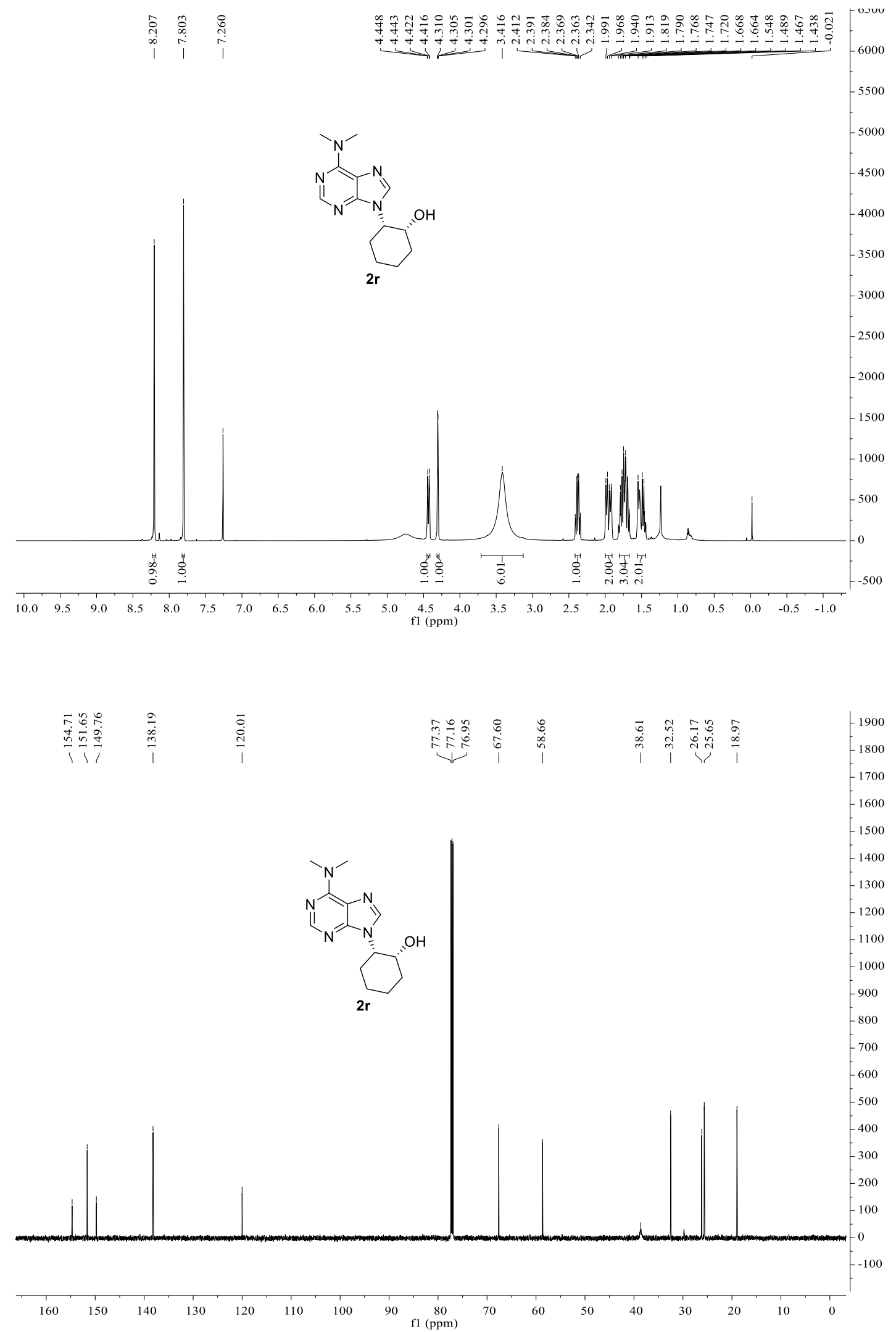


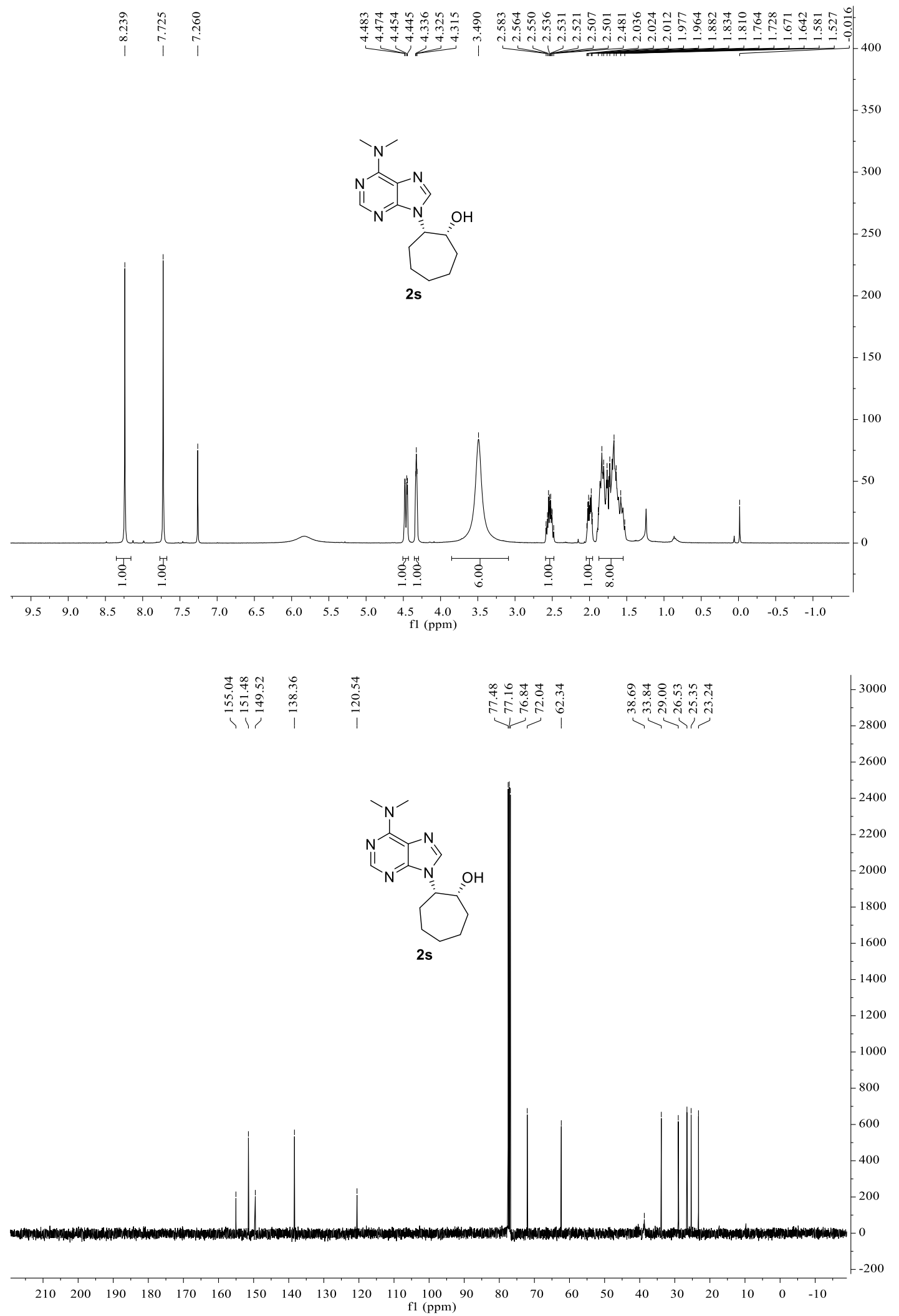




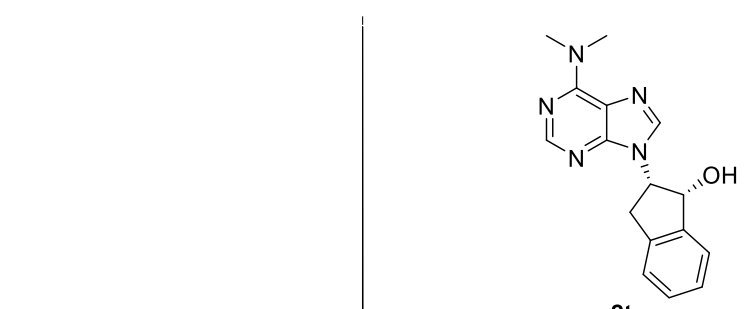

$2 t$
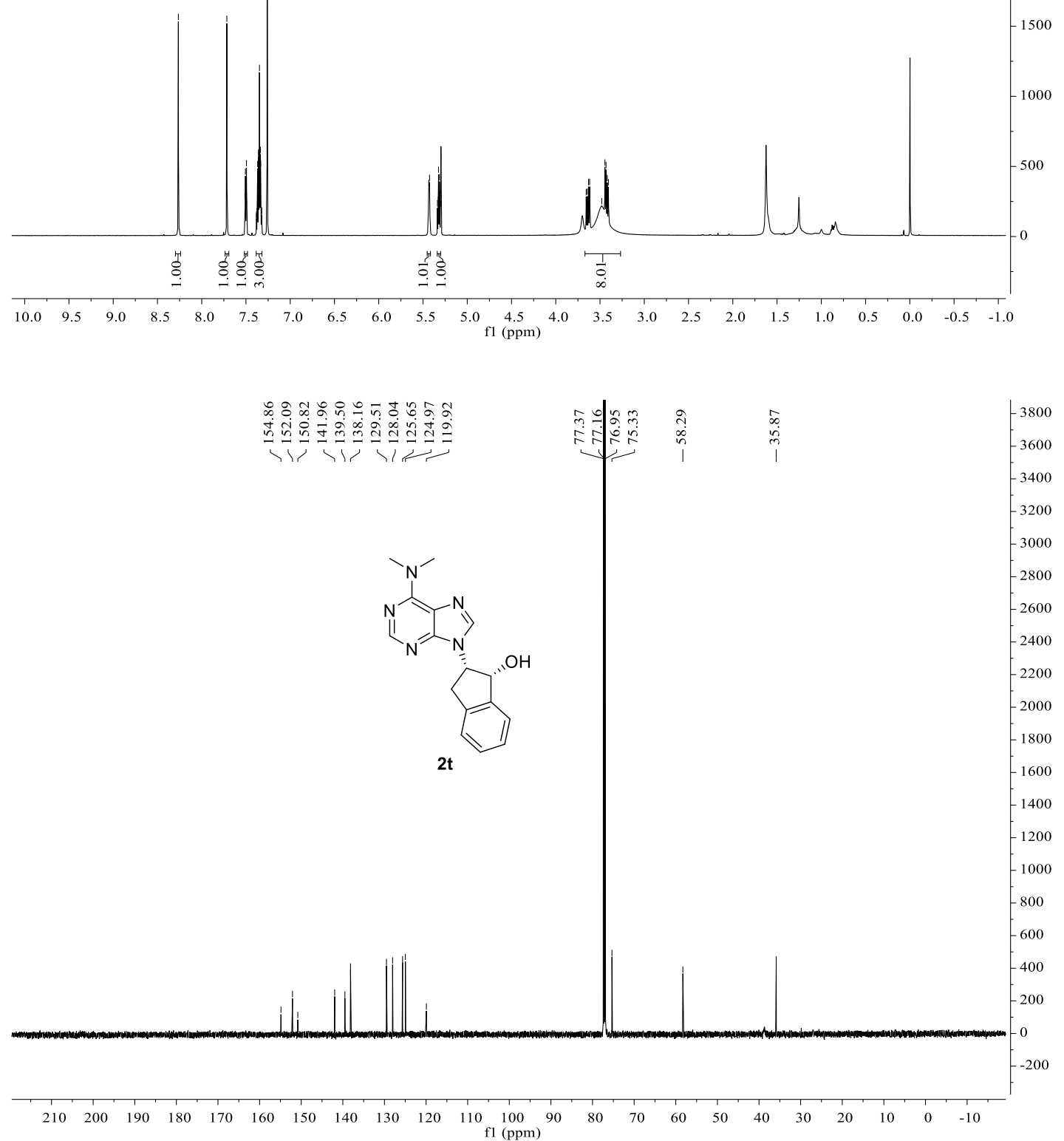


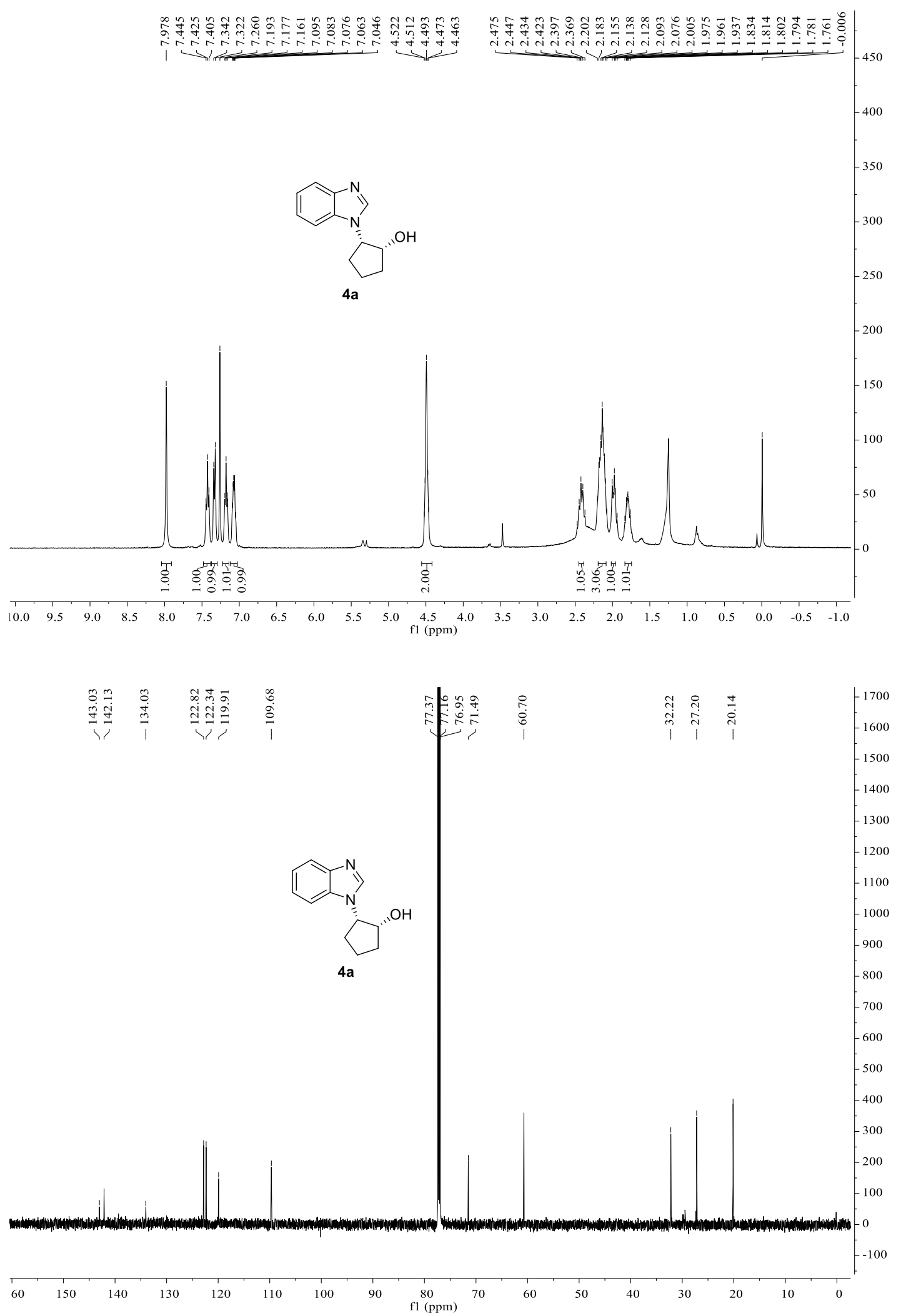




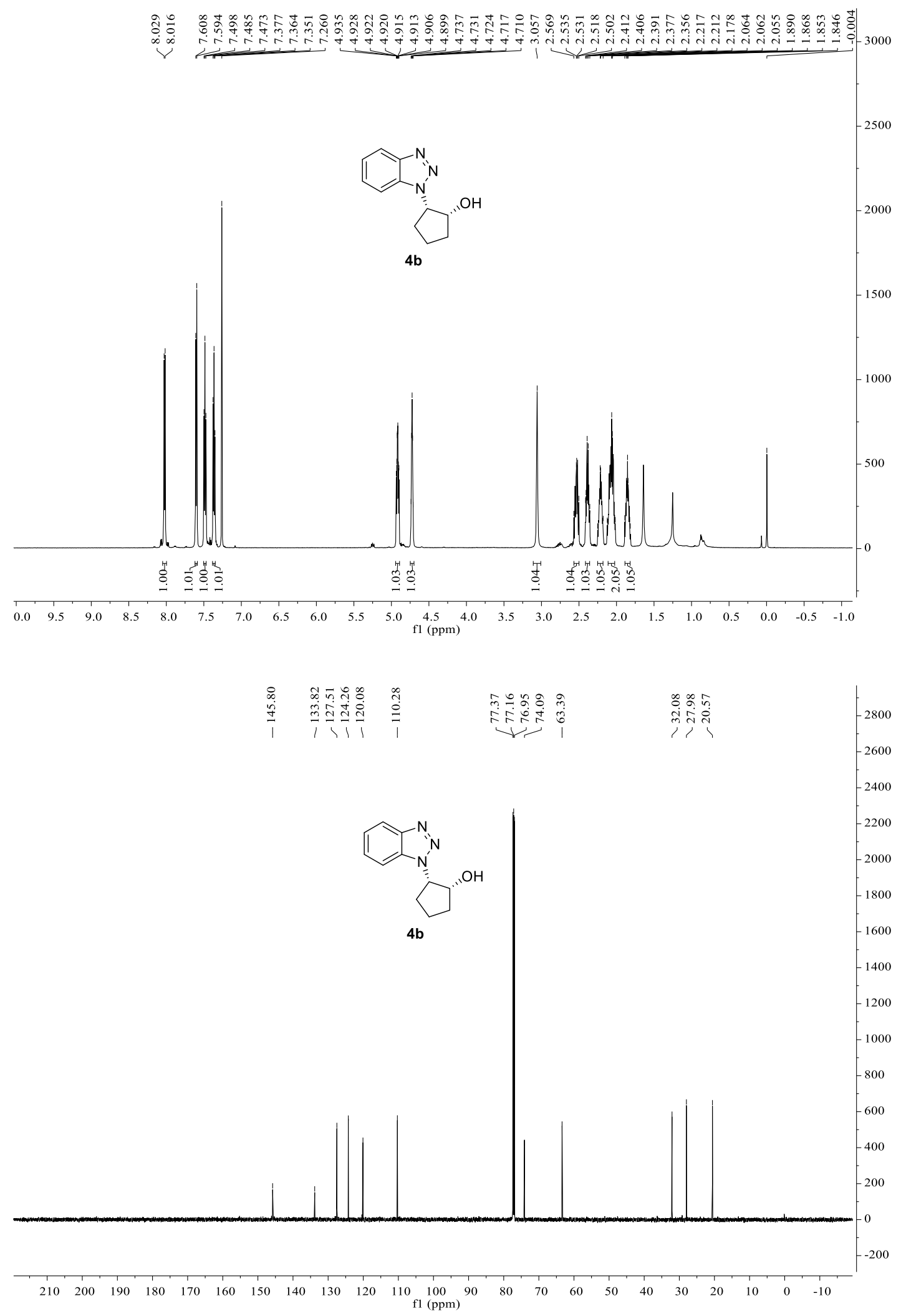




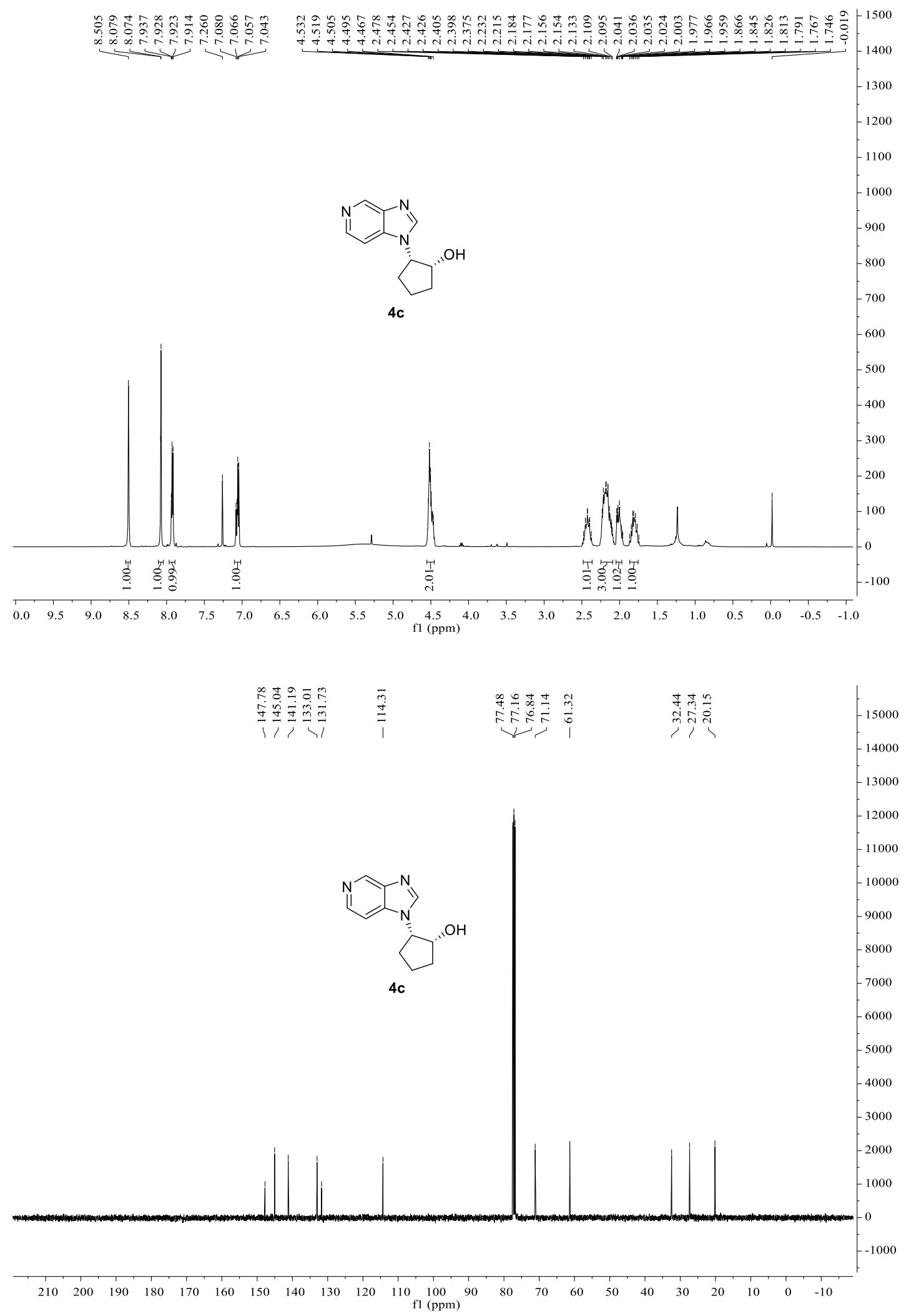



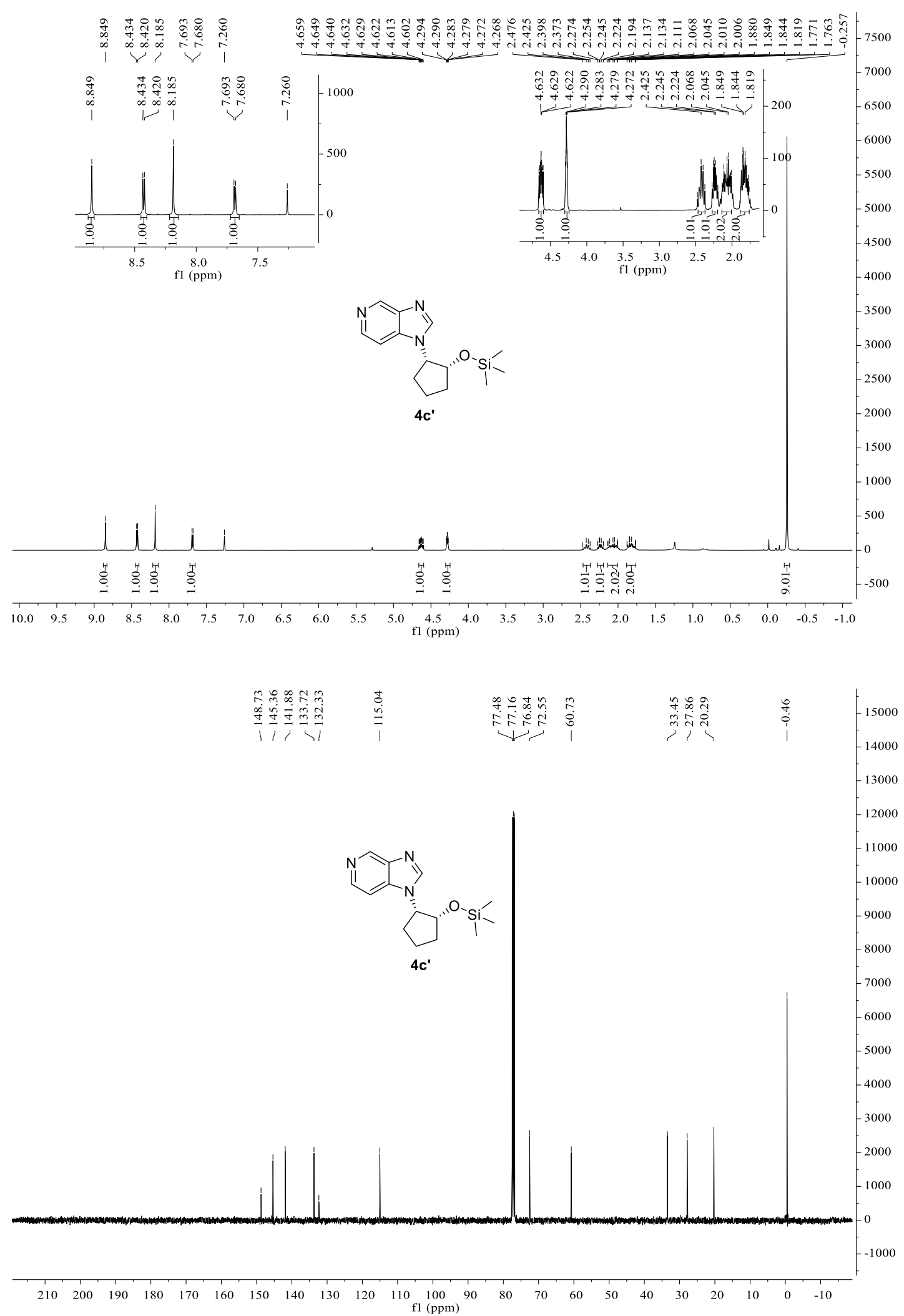


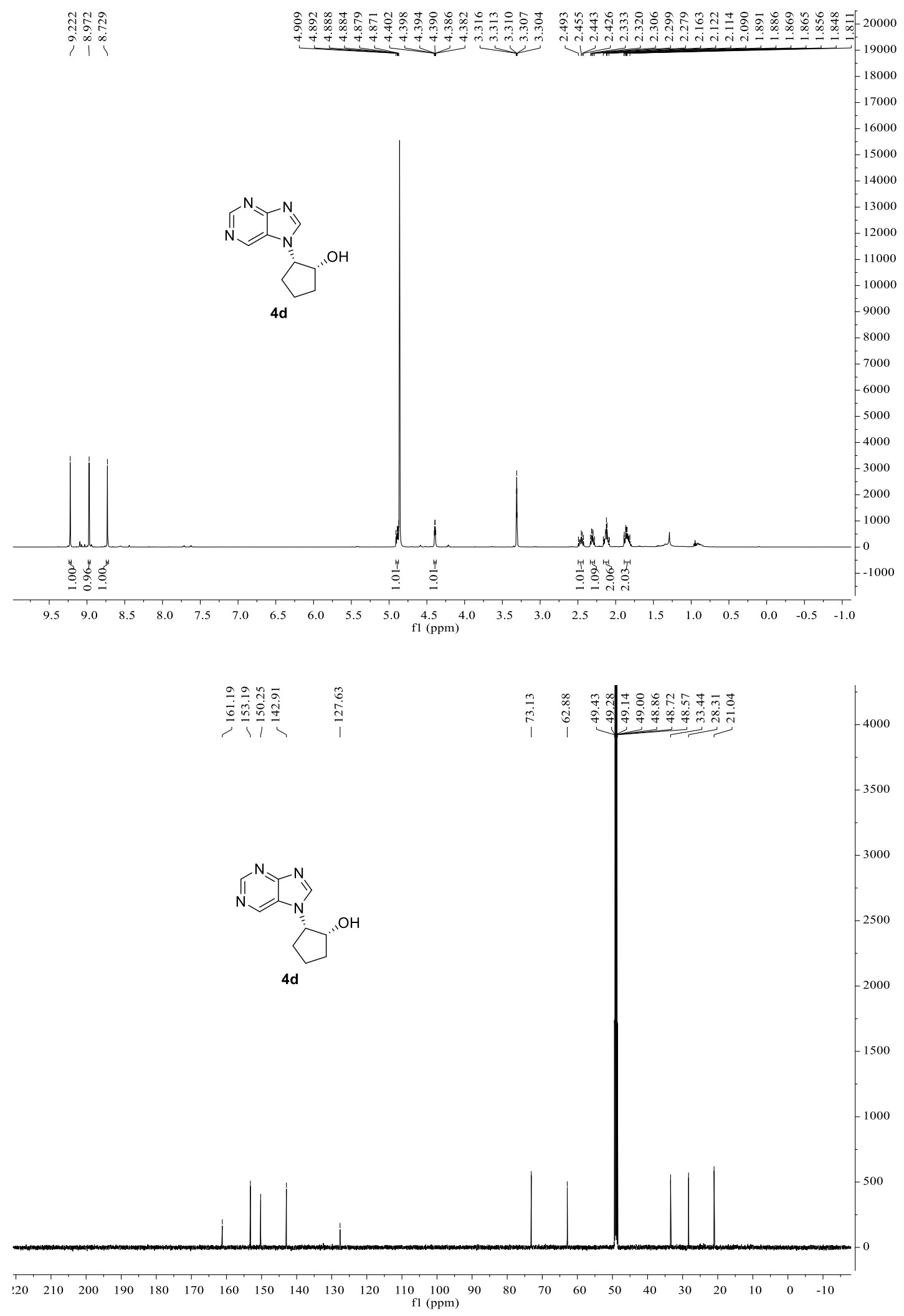



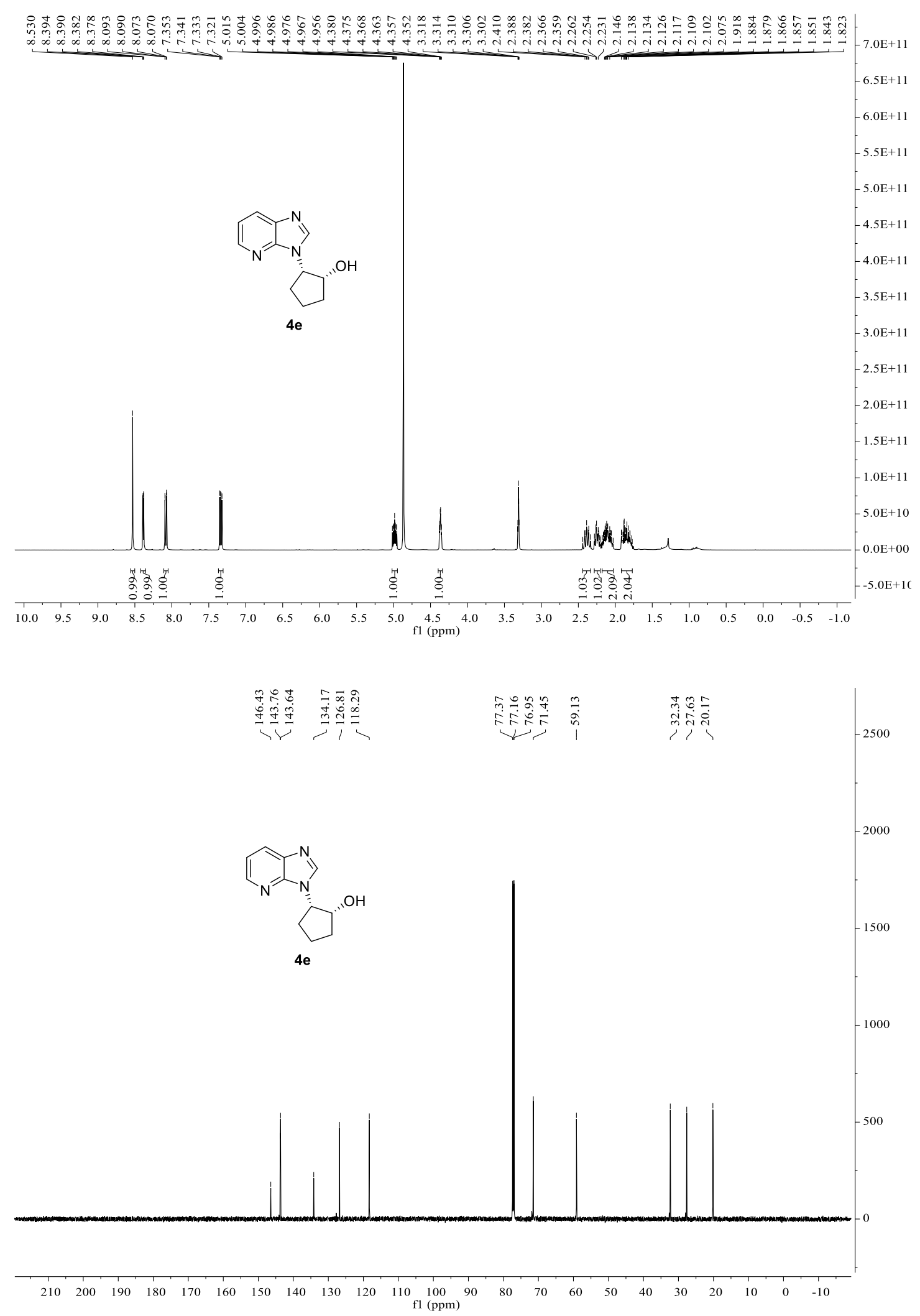


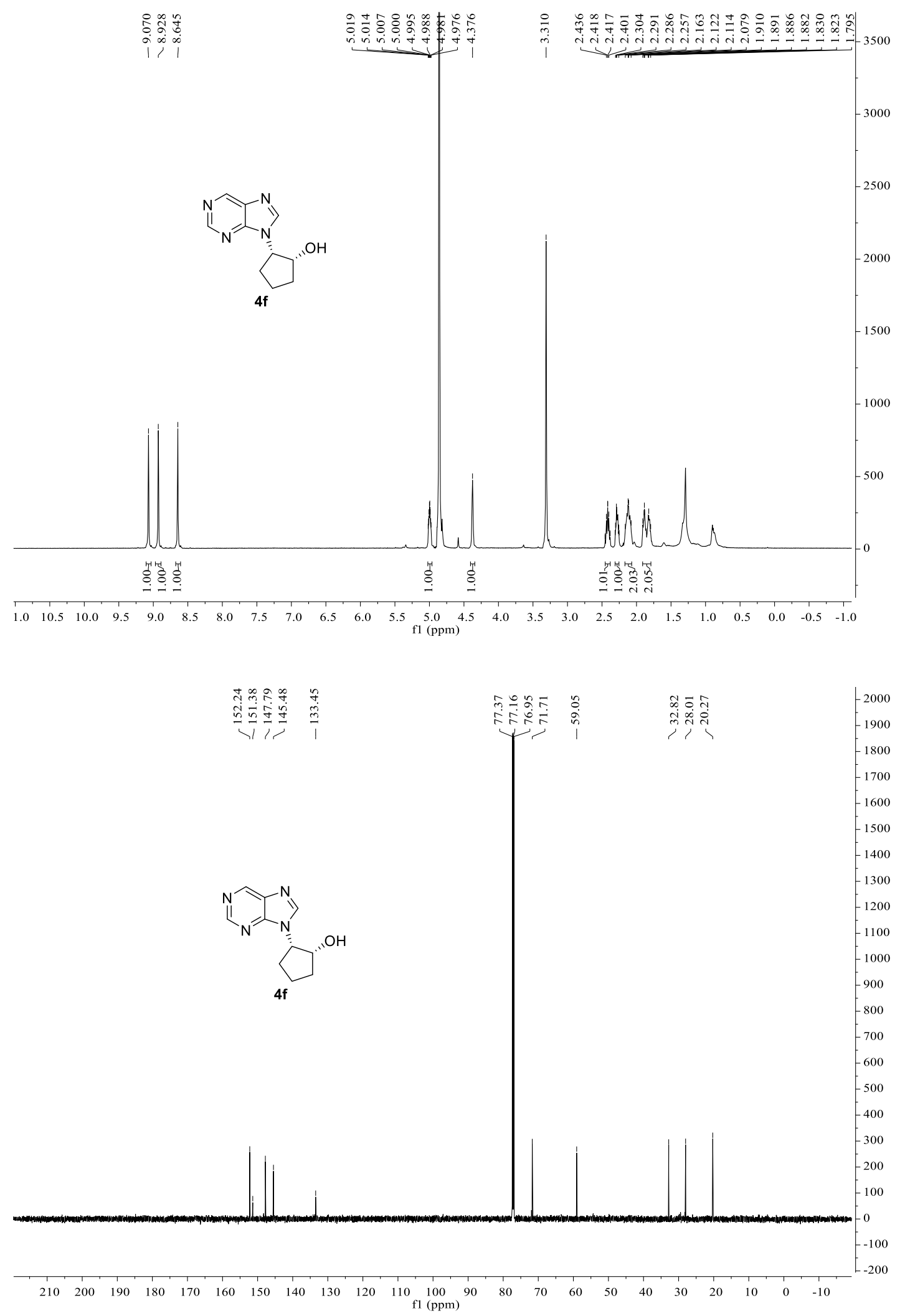




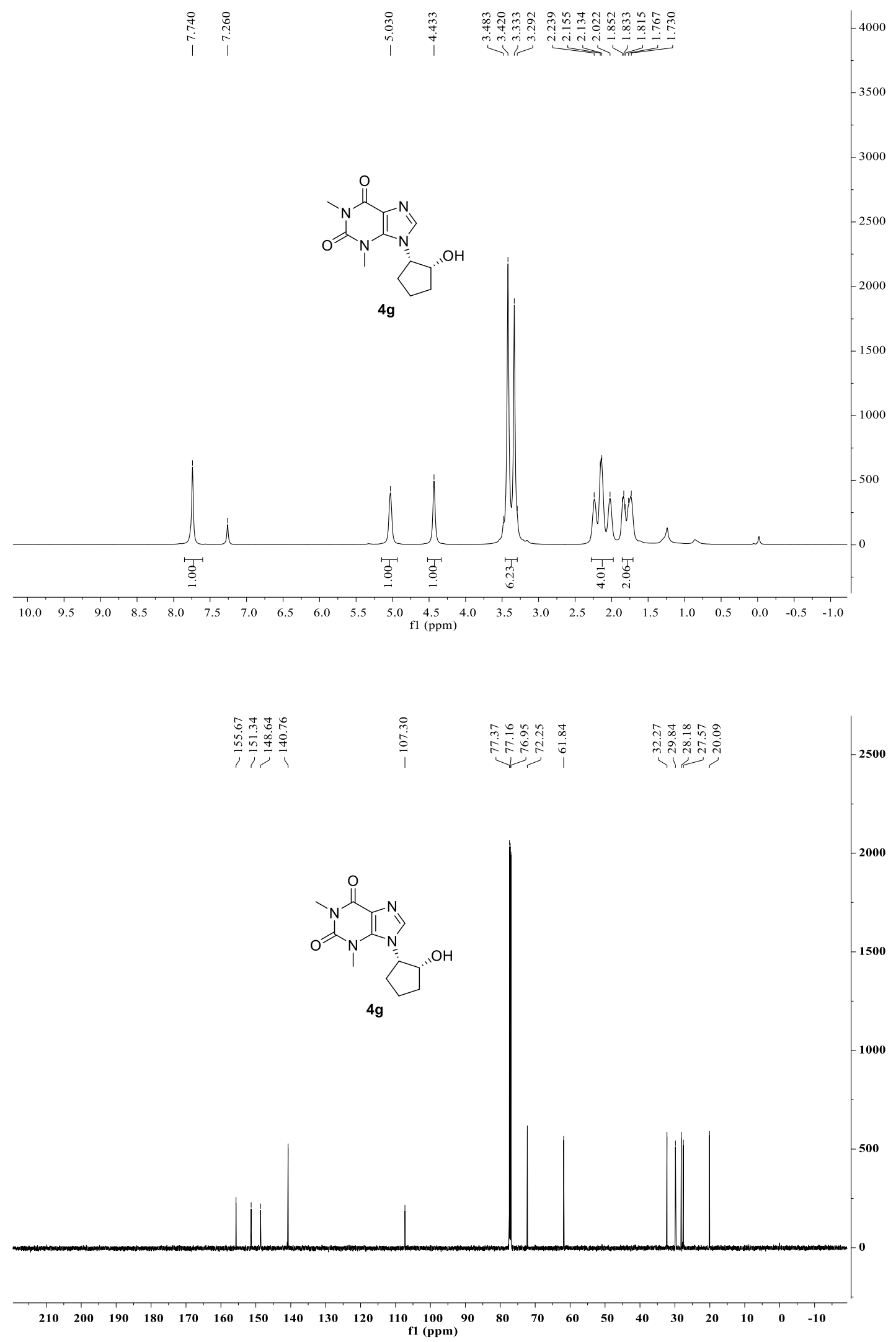




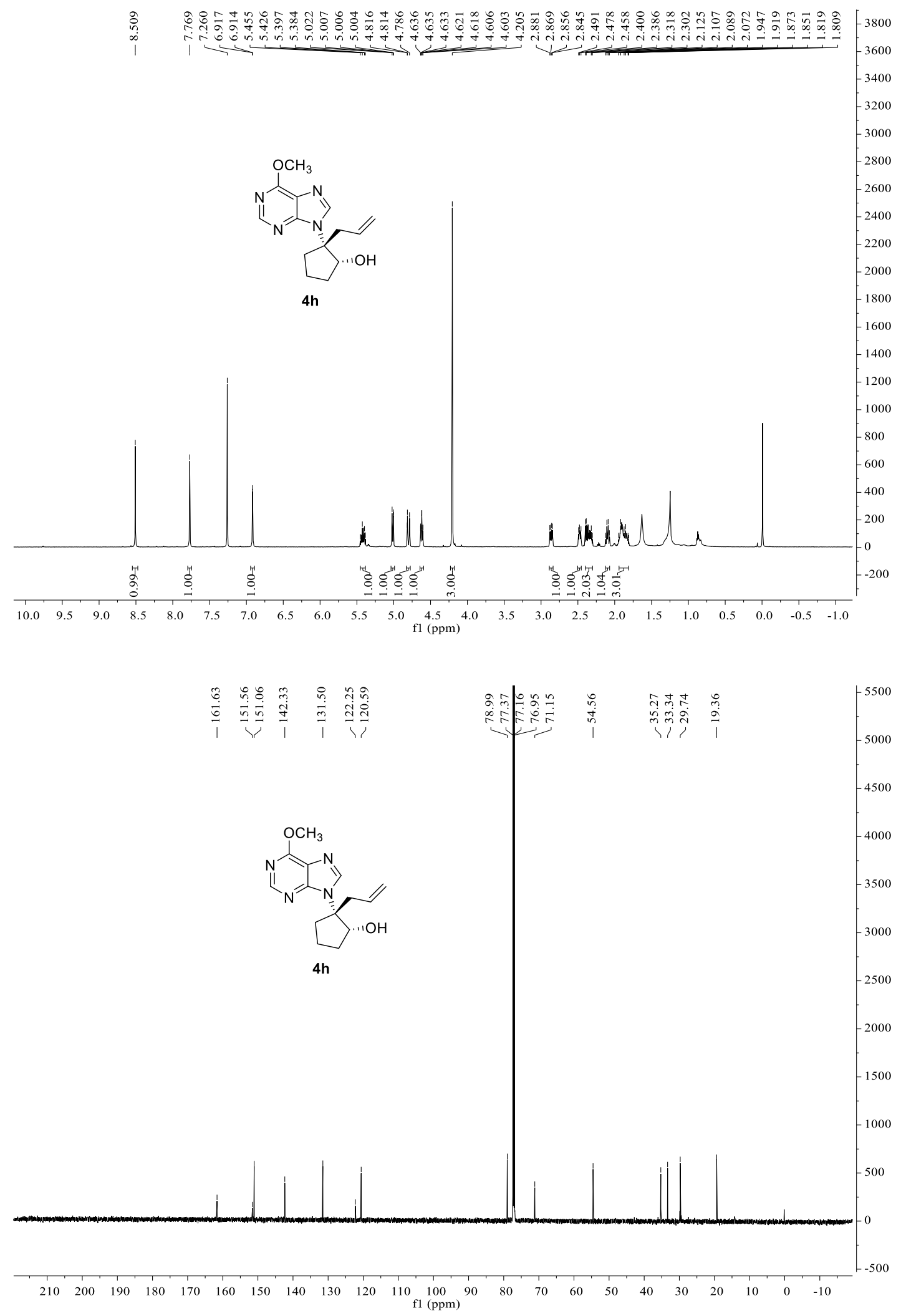




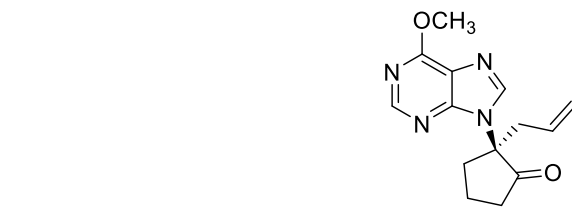

(S)-3h
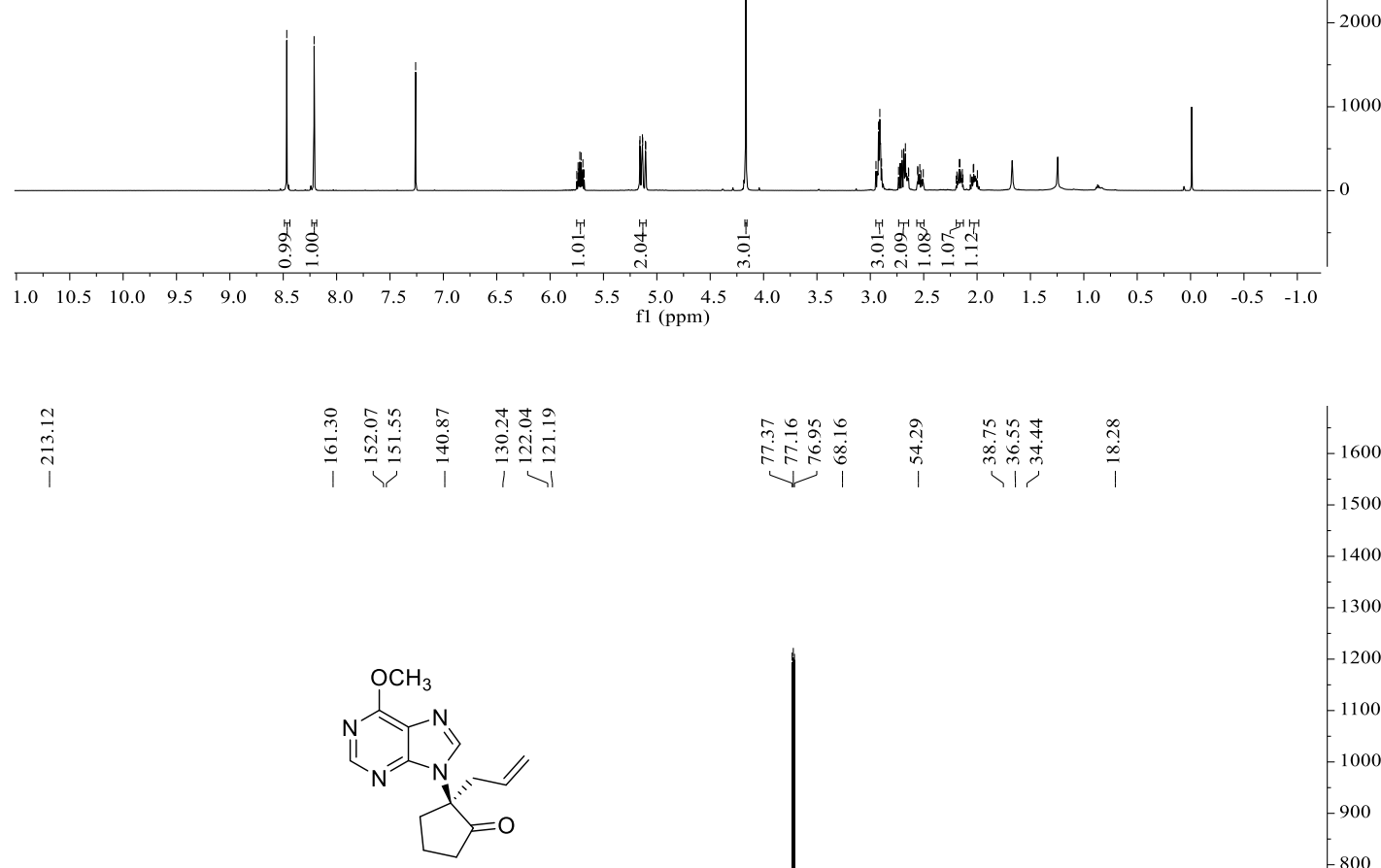

(S)-3h

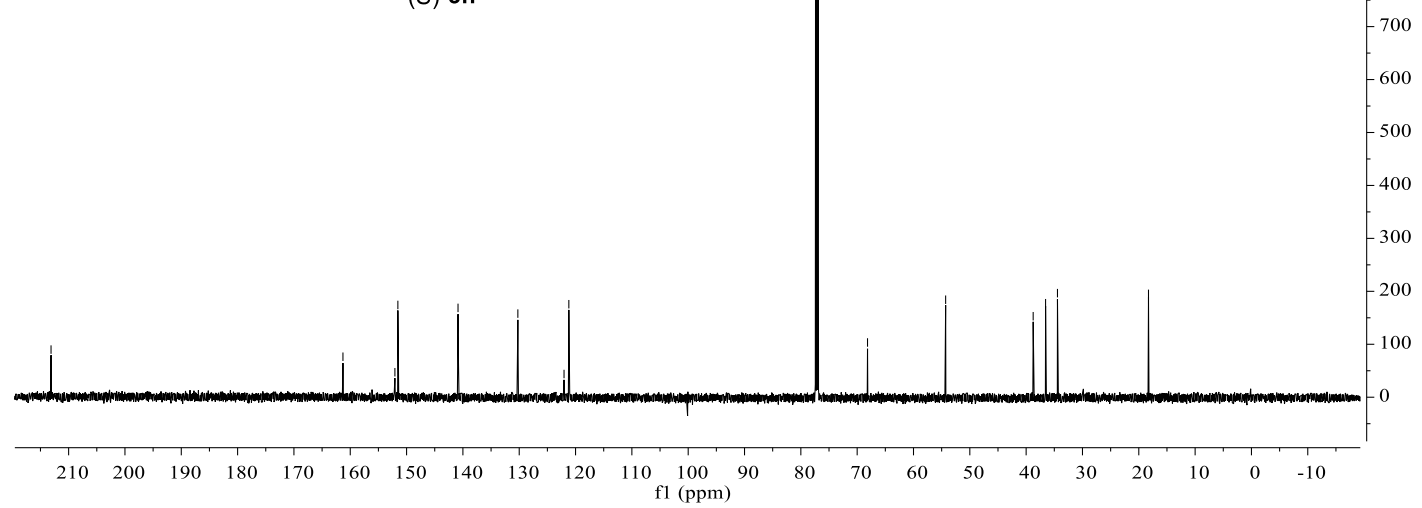


(3) Copies of NMR spectra of the derivatives

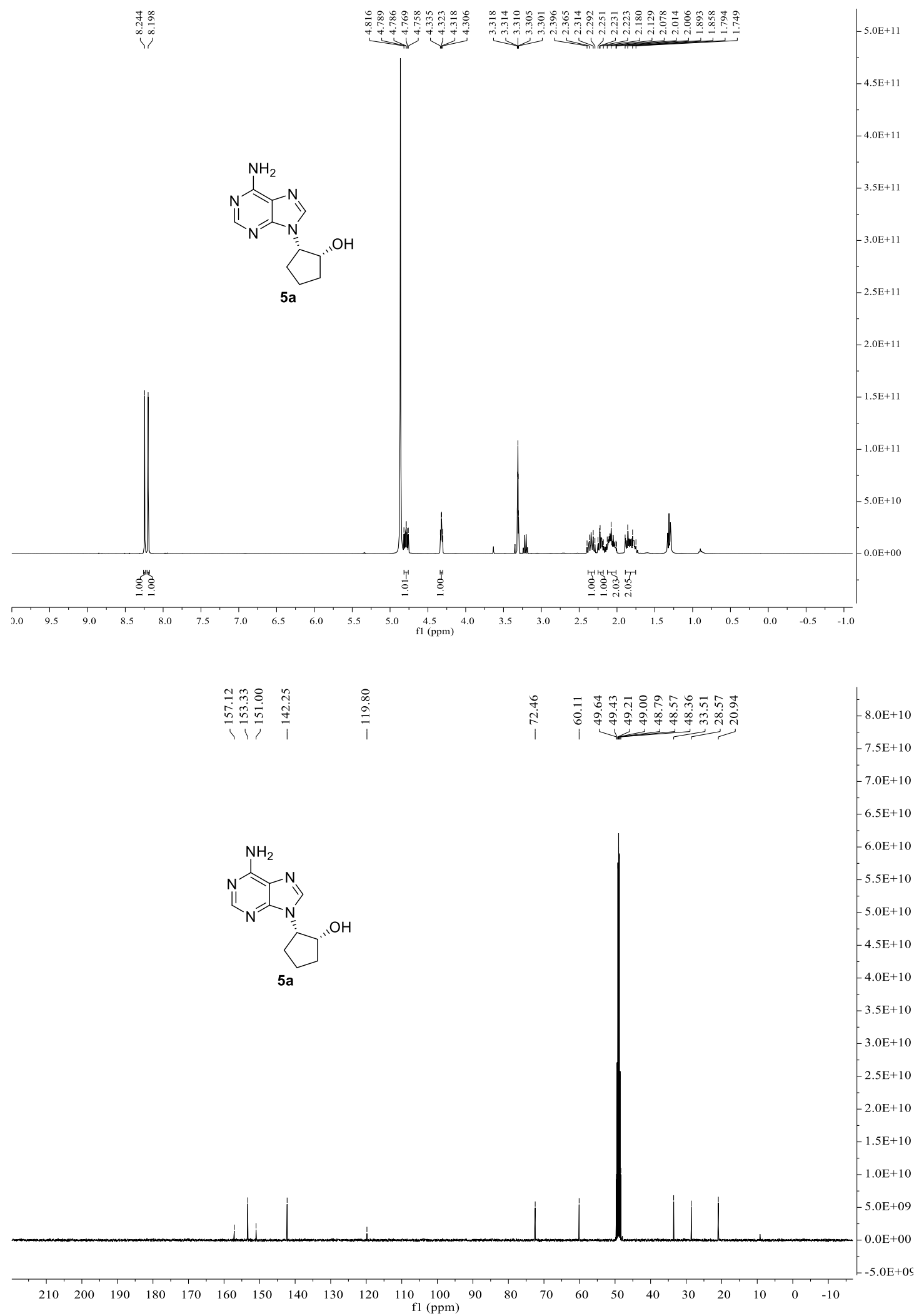




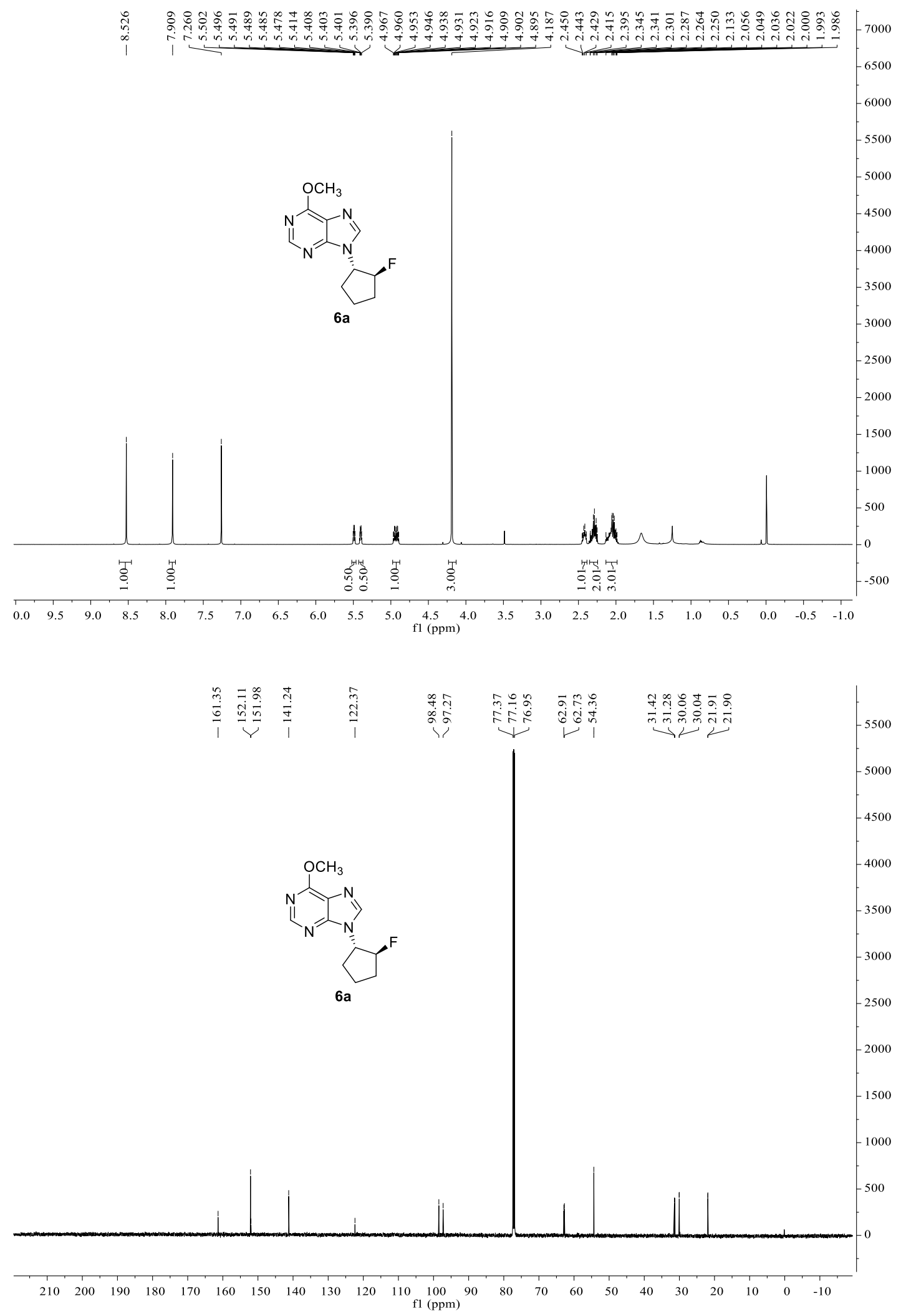




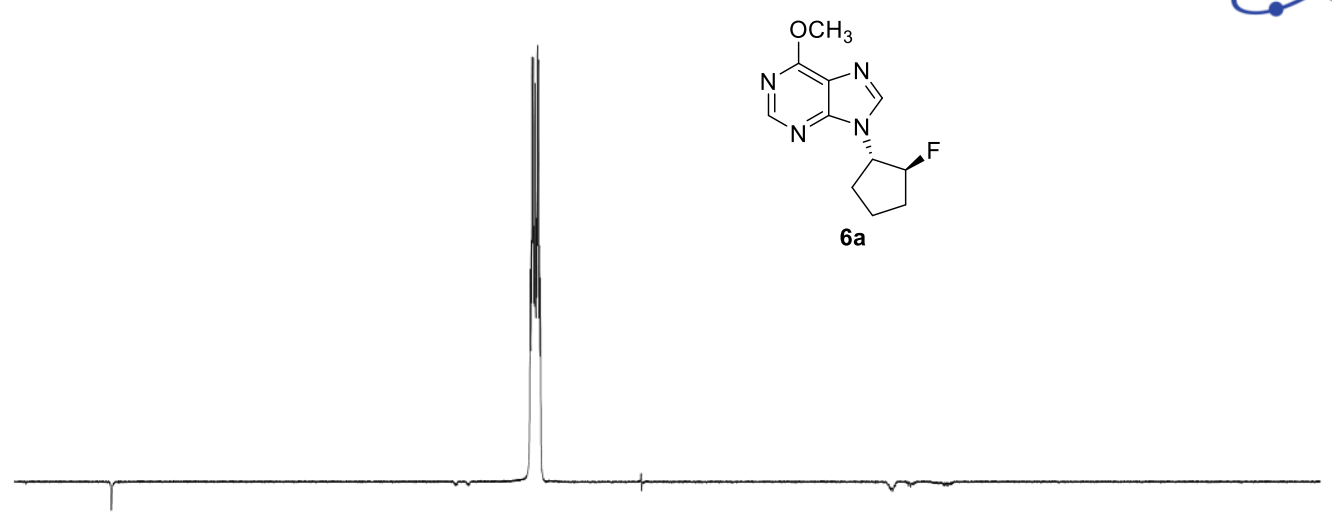

$\begin{array}{llllllllllll} & 8 & 7 & 6 & 5 & 4 & 3 & 2 & 1 & 0 & \mathrm{ppm}\end{array}$

F19 No:2

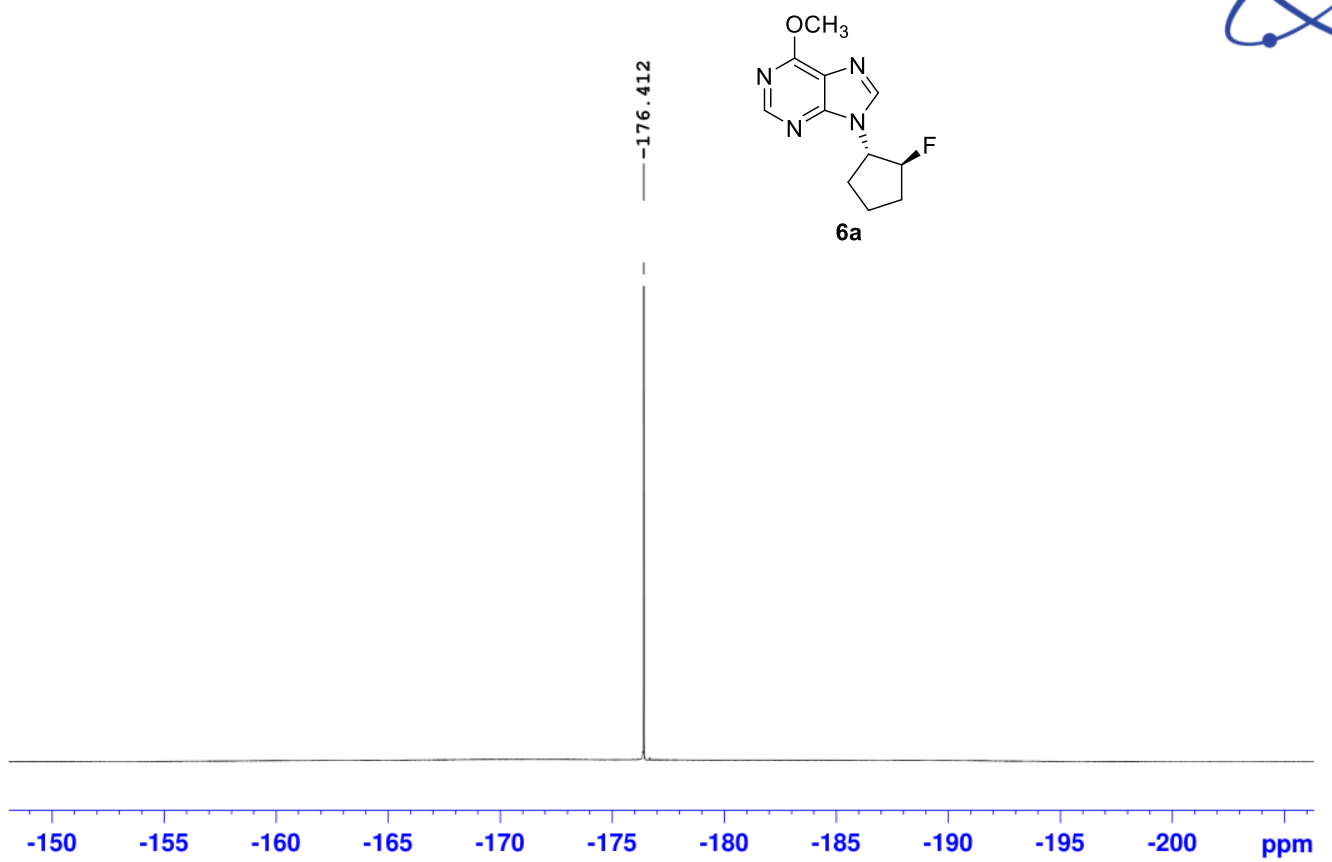

BRUKER 

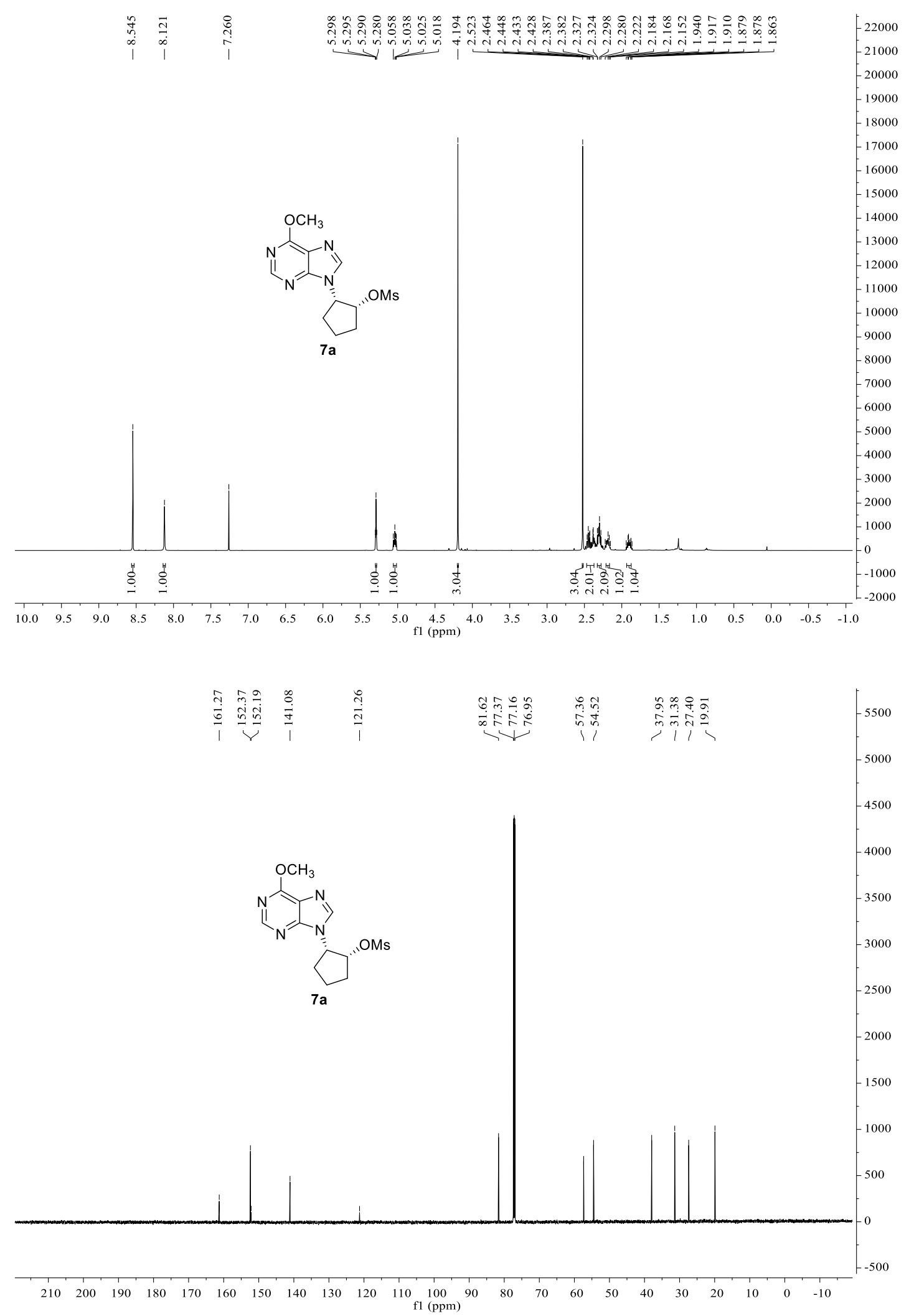


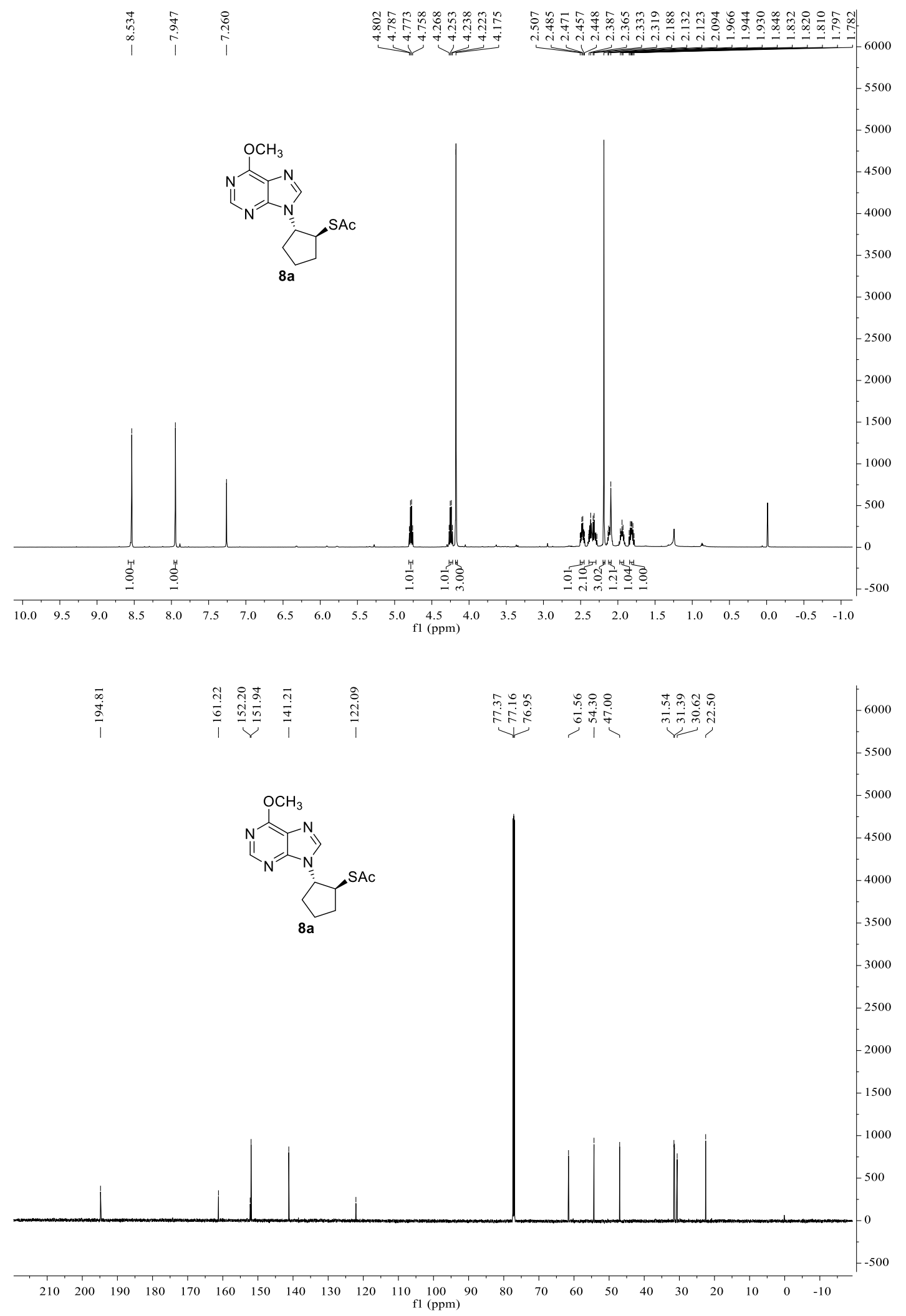




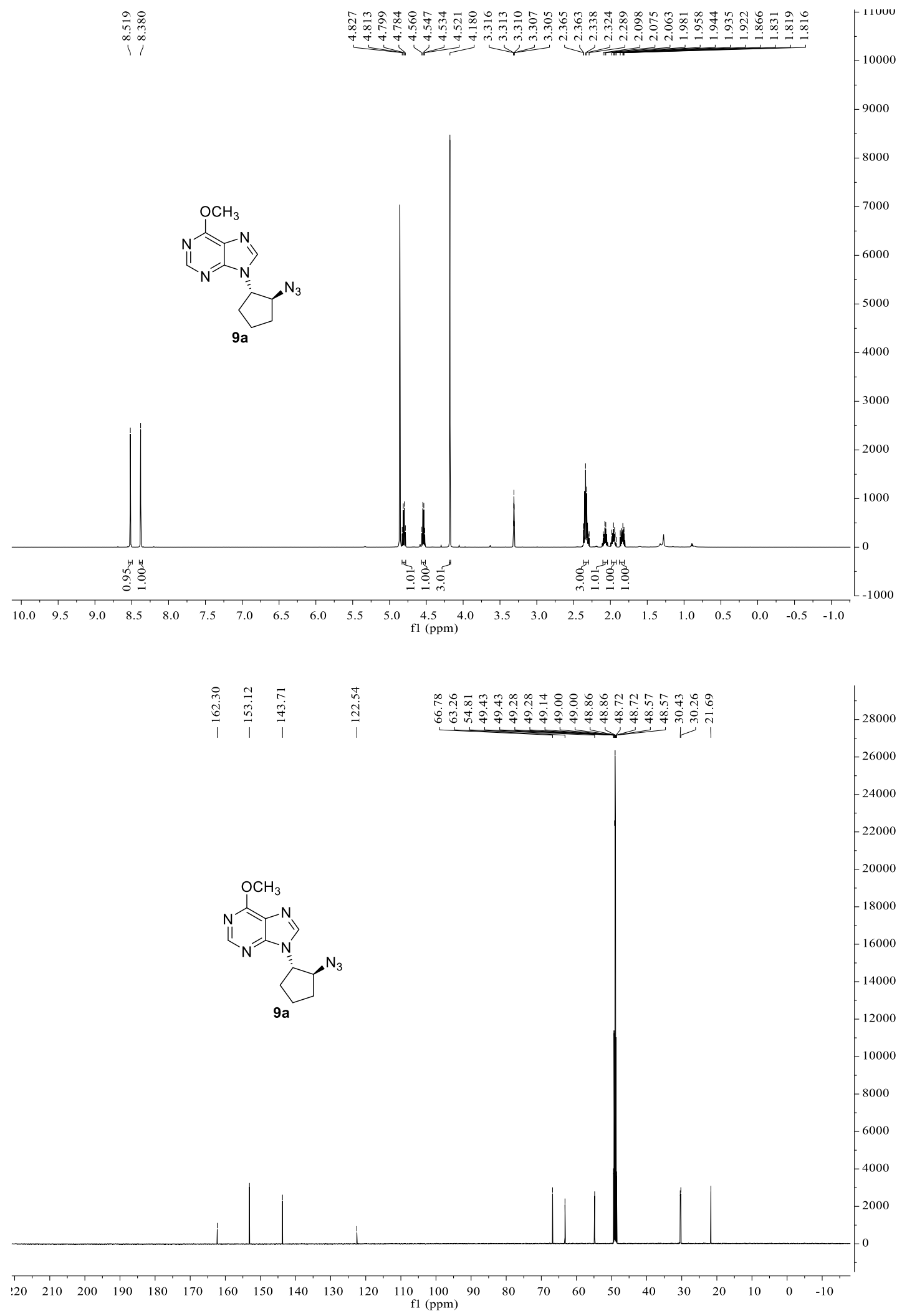




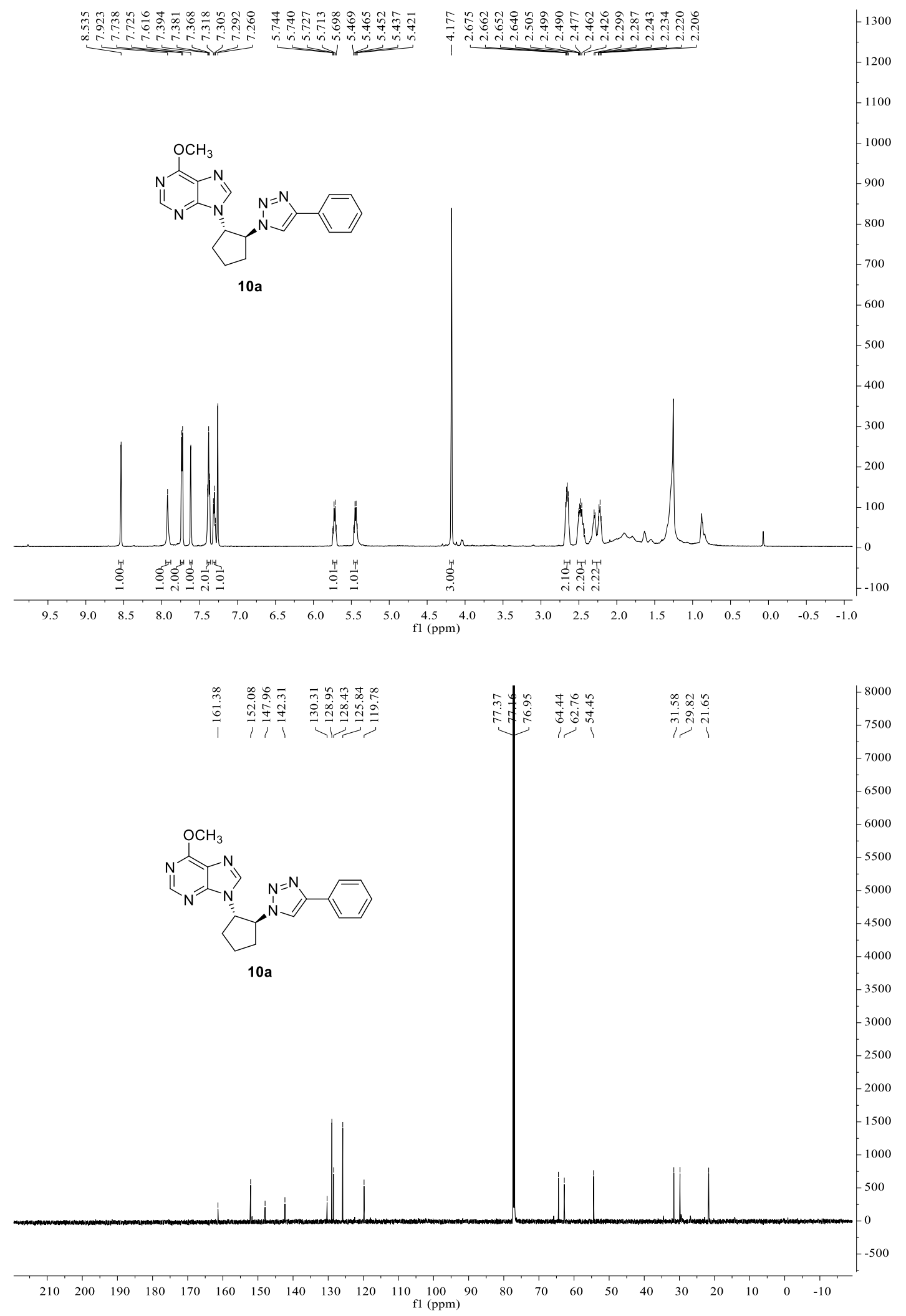




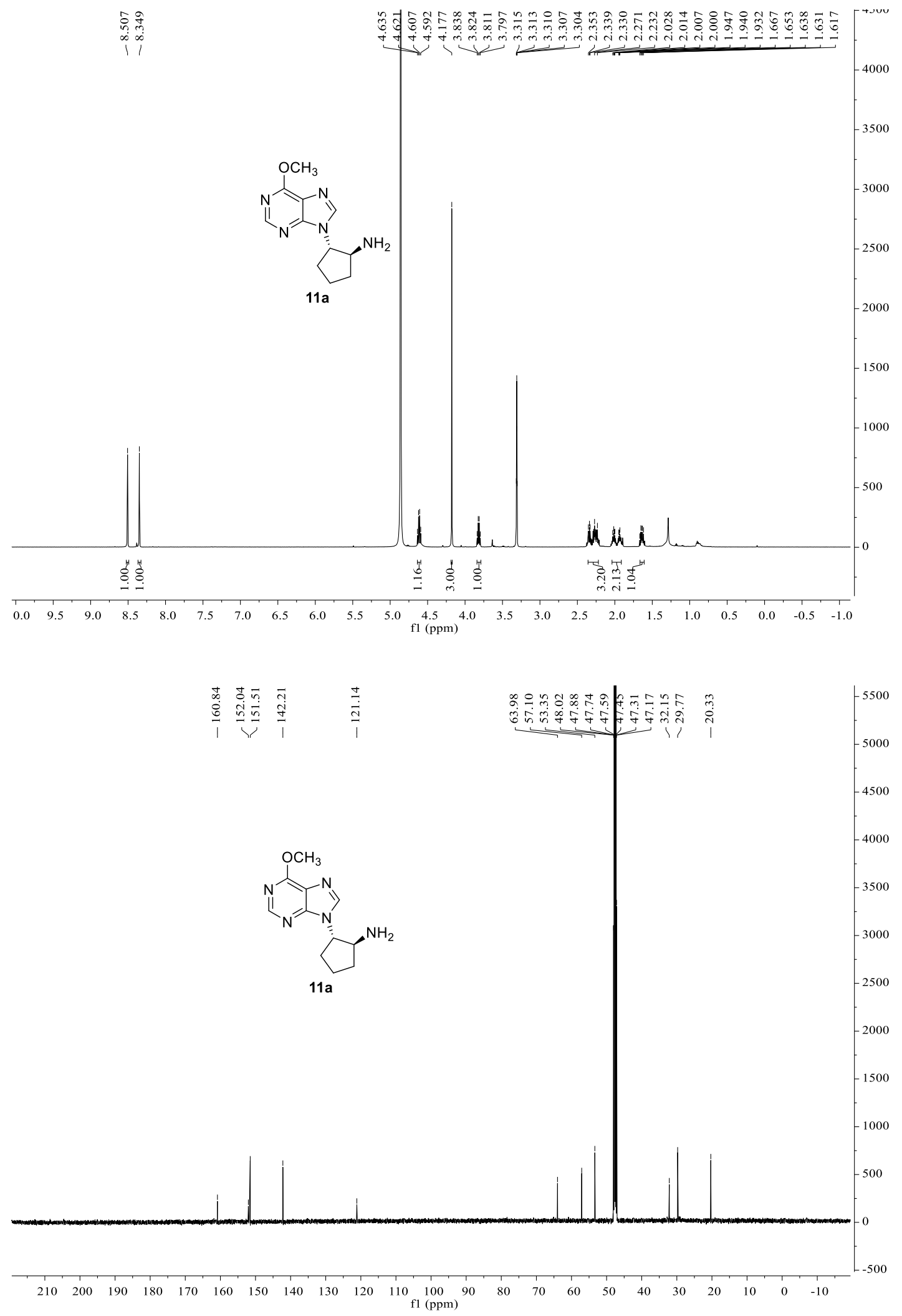


10. Copies of HPLC spectra for racemic and chiral products

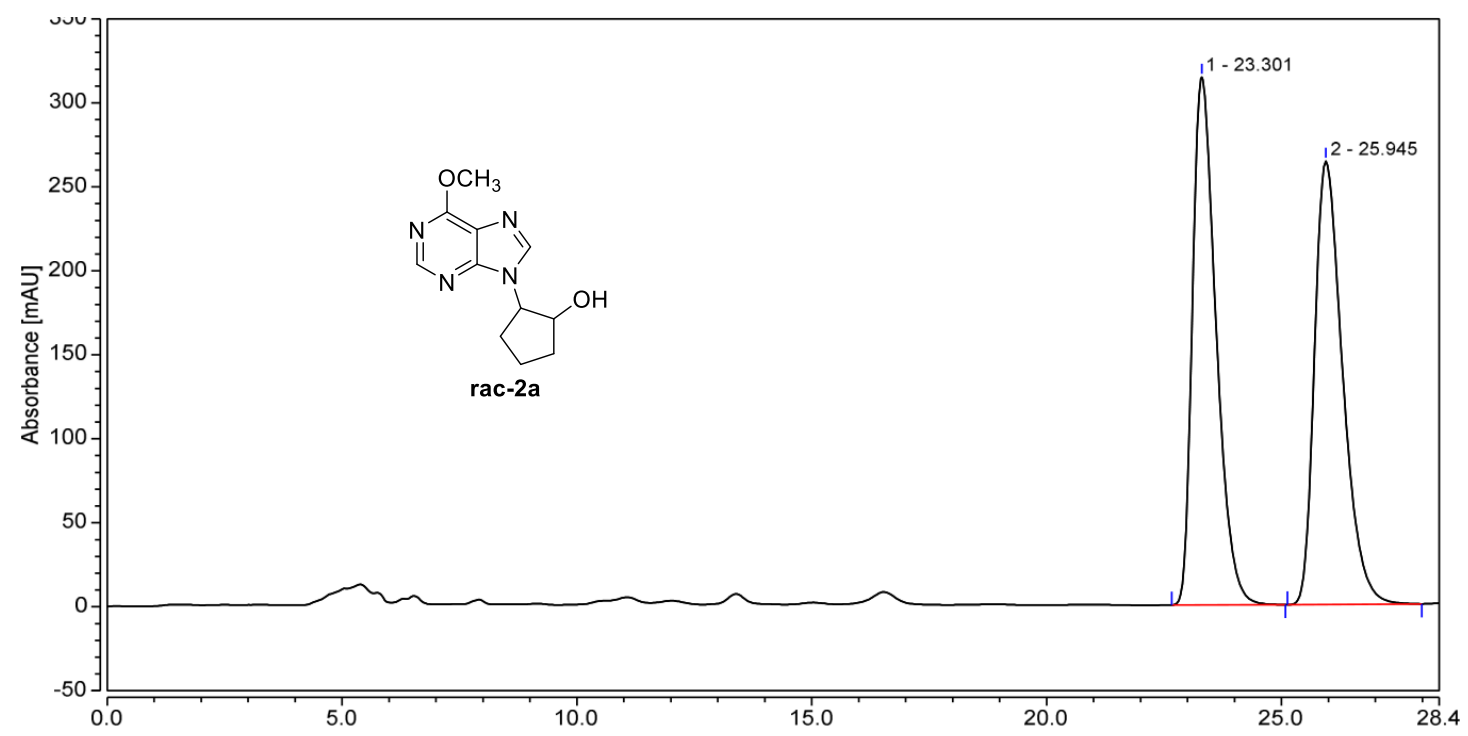

\begin{tabular}{|l|c|c|c|c|}
\hline Peak & $\begin{array}{c}\text { Retention Time } \\
\text { min }\end{array}$ & $\begin{array}{c}\text { Area } \\
\mathrm{mAU}^{*} \min \end{array}$ & $\begin{array}{c}\text { Height } \\
\mathrm{mAU}\end{array}$ & $\begin{array}{c}\text { Area } \\
\%\end{array}$ \\
\hline 1 & 23.301 & 183.254 & 314.316 & 50.06 \\
2 & 25.945 & 182.834 & 263.990 & 49.94 \\
\hline \multicolumn{2}{l|}{ Total: } & $\mathbf{3 6 6 . 0 8 8}$ & $\mathbf{5 7 8 . 3 0 6}$ & $\mathbf{1 0 0 . 0 0}$ \\
\hline
\end{tabular}

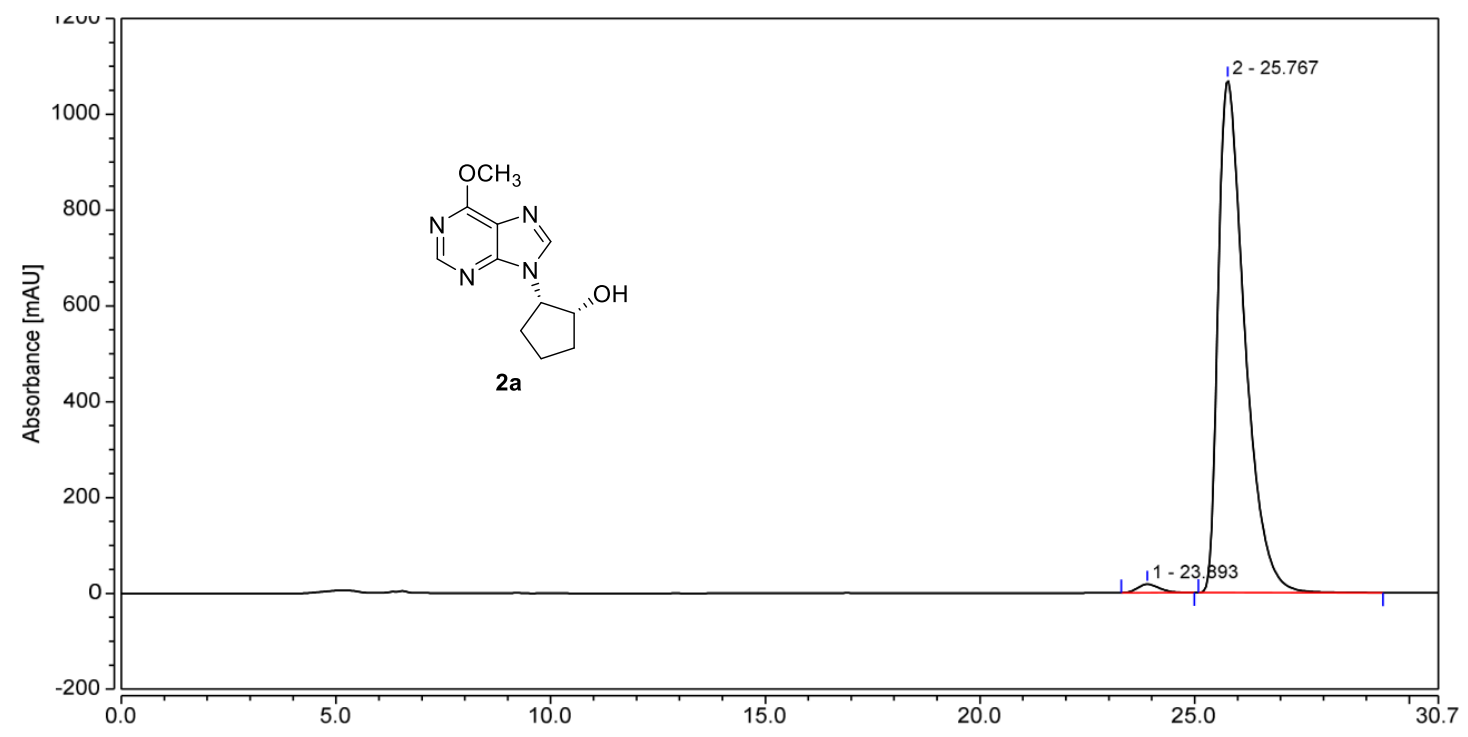

\begin{tabular}{|l|c|c|c|c|}
\hline Peak & $\begin{array}{c}\text { Retention Time } \\
\mathrm{min}\end{array}$ & $\begin{array}{c}\text { Area } \\
\mathrm{mAU}^{*} \min \end{array}$ & $\begin{array}{c}\text { Height } \\
\mathrm{mAU}\end{array}$ & $\begin{array}{c}\text { Area } \\
\%\end{array}$ \\
\hline 1 & 23.893 & 10.683 & 17.805 & 1.37 \\
2 & 25.767 & 771.486 & 1069.941 & 98.63 \\
\hline \multicolumn{2}{l|}{ Total: } & $\mathbf{7 8 2 . 1 6 9}$ & $\mathbf{1 0 8 7 . 7 4 6}$ & $\mathbf{1 0 0 . 0 0}$ \\
\hline
\end{tabular}




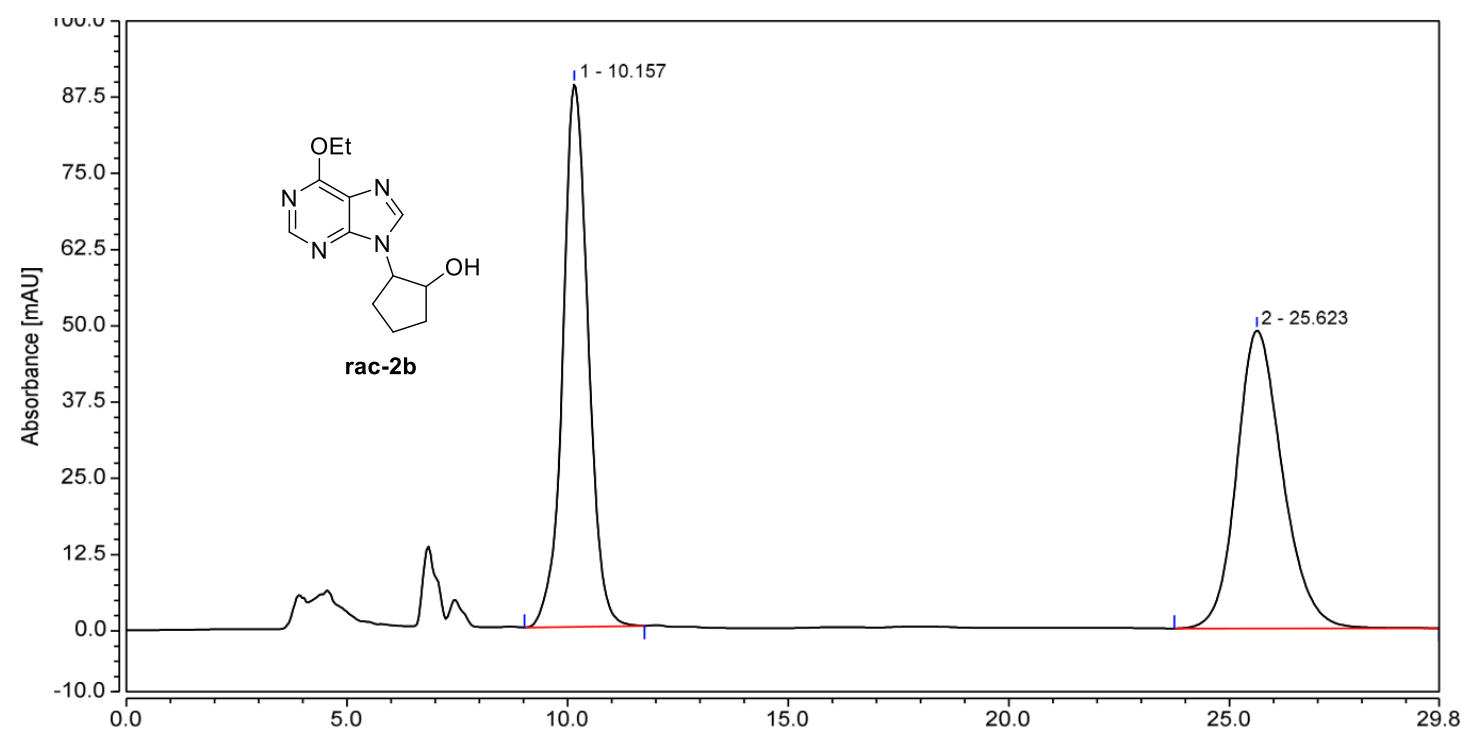

\begin{tabular}{|l|c|c|c|c|}
\hline Peak & $\begin{array}{c}\text { Retention Time } \\
\min \end{array}$ & $\begin{array}{c}\text { Area } \\
\mathrm{mAU}^{*} \min \end{array}$ & $\begin{array}{c}\text { Height } \\
\mathrm{mAU}\end{array}$ & $\begin{array}{c}\text { Area } \\
\%\end{array}$ \\
\hline 1 & 10.157 & 59.337 & 89.021 & 50.25 \\
2 & 25.623 & 58.737 & 48.859 & 49.75 \\
\hline \multicolumn{2}{l|}{ Total: } & $\mathbf{1 1 8 . 0 7 4}$ & $\mathbf{1 3 7 . 8 8 0}$ & $\mathbf{1 0 0 . 0 0}$ \\
\hline
\end{tabular}

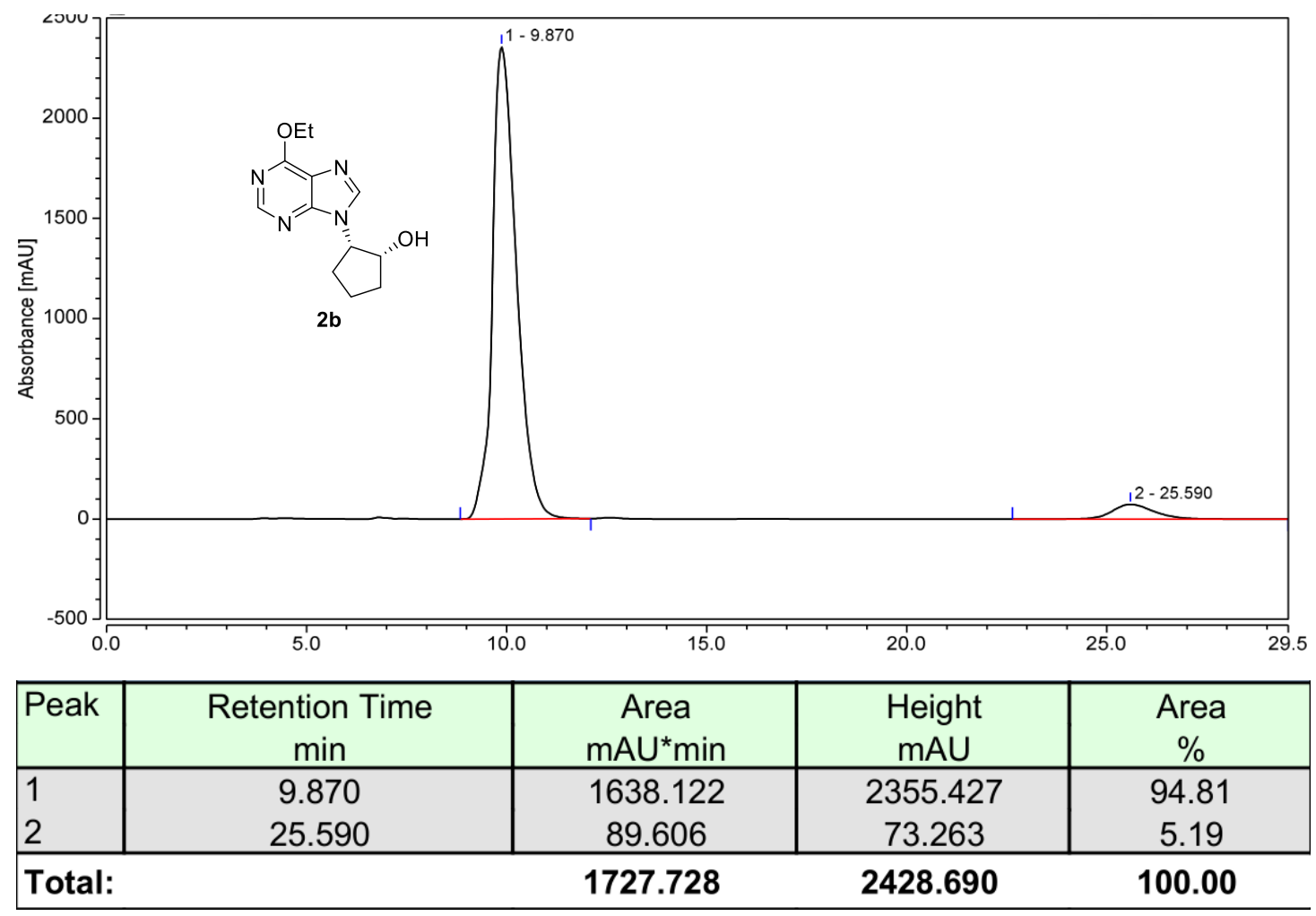




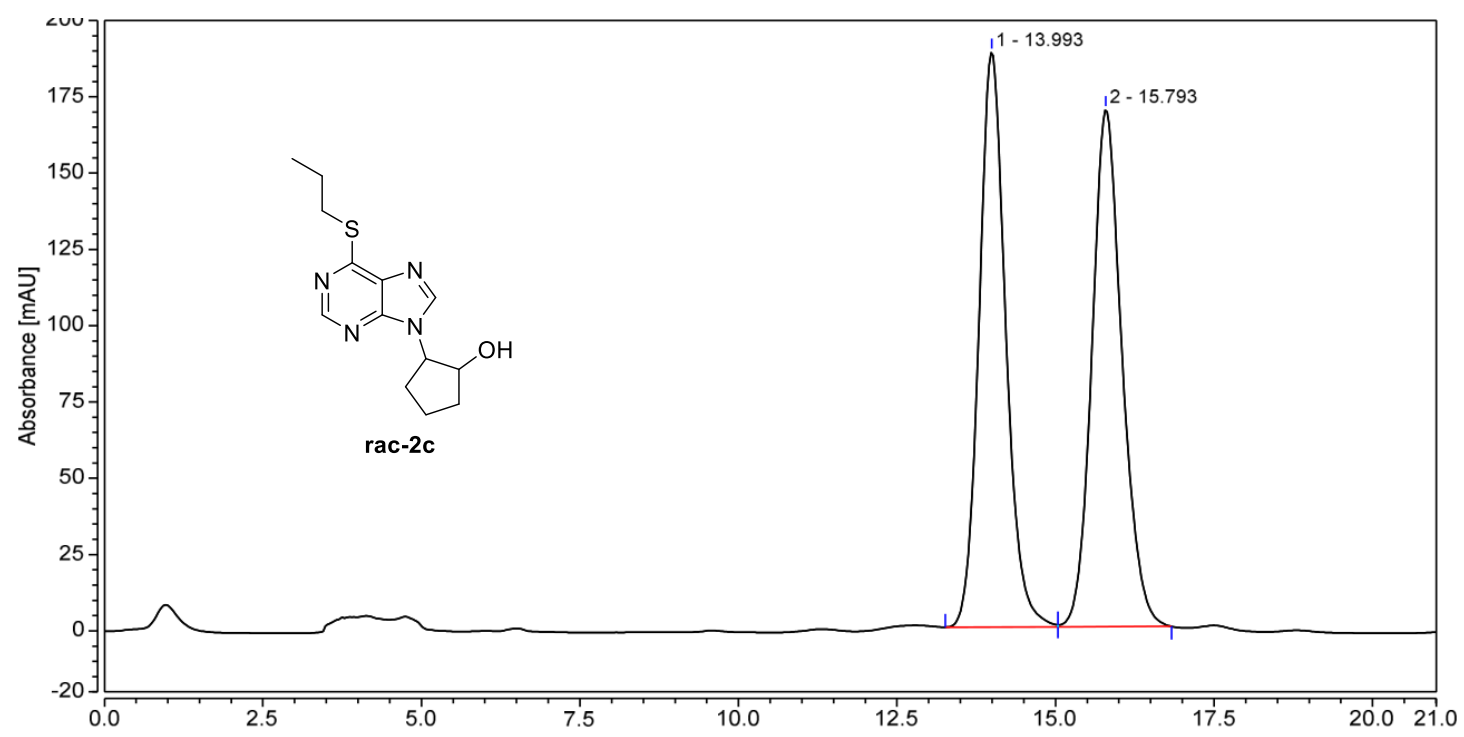

\begin{tabular}{|l|c|c|c|c|}
\hline Peak & $\begin{array}{c}\text { Retention Time } \\
\mathrm{min}\end{array}$ & $\begin{array}{c}\text { Area } \\
\mathrm{mAU}^{*} \min \end{array}$ & $\begin{array}{c}\text { Height } \\
\mathrm{mAU}\end{array}$ & $\begin{array}{c}\text { Area } \\
\%\end{array}$ \\
\hline 1 & 13.993 & 92.028 & 188.476 & 49.64 \\
2 & 15.793 & 93.360 & 169.480 & 50.36 \\
\hline \multicolumn{2}{l|}{ Total: } & $\mathbf{1 8 5 . 3 8 8}$ & $\mathbf{3 5 7 . 9 5 5}$ & $\mathbf{1 0 0 . 0 0}$ \\
\hline
\end{tabular}

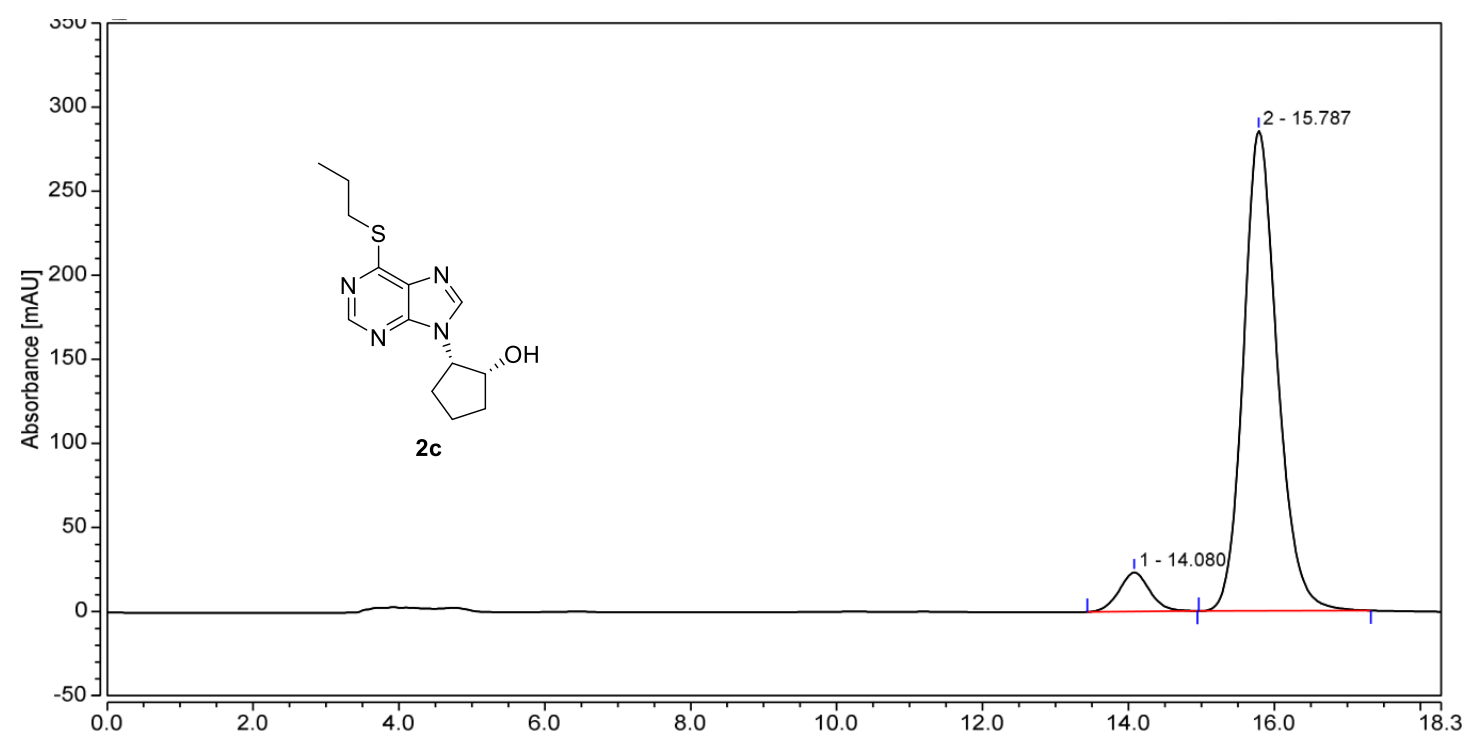

\begin{tabular}{|l|c|c|c|c|}
\hline Peak & $\begin{array}{c}\text { Retention Time } \\
\text { min }\end{array}$ & $\begin{array}{c}\text { Area } \\
\mathrm{mAU}^{*} \min \end{array}$ & $\begin{array}{c}\text { Height } \\
\mathrm{mAU}\end{array}$ & $\begin{array}{c}\text { Area } \\
\%\end{array}$ \\
\hline 1 & 14.080 & 11.391 & 23.214 & 6.91 \\
2 & 15.787 & 153.486 & 285.375 & 93.09 \\
\hline Total: & $\mathbf{1 6 4 . 8 7 7}$ & $\mathbf{3 0 8 . 5 8 9}$ & $\mathbf{1 0 0 . 0 0}$ \\
\hline
\end{tabular}




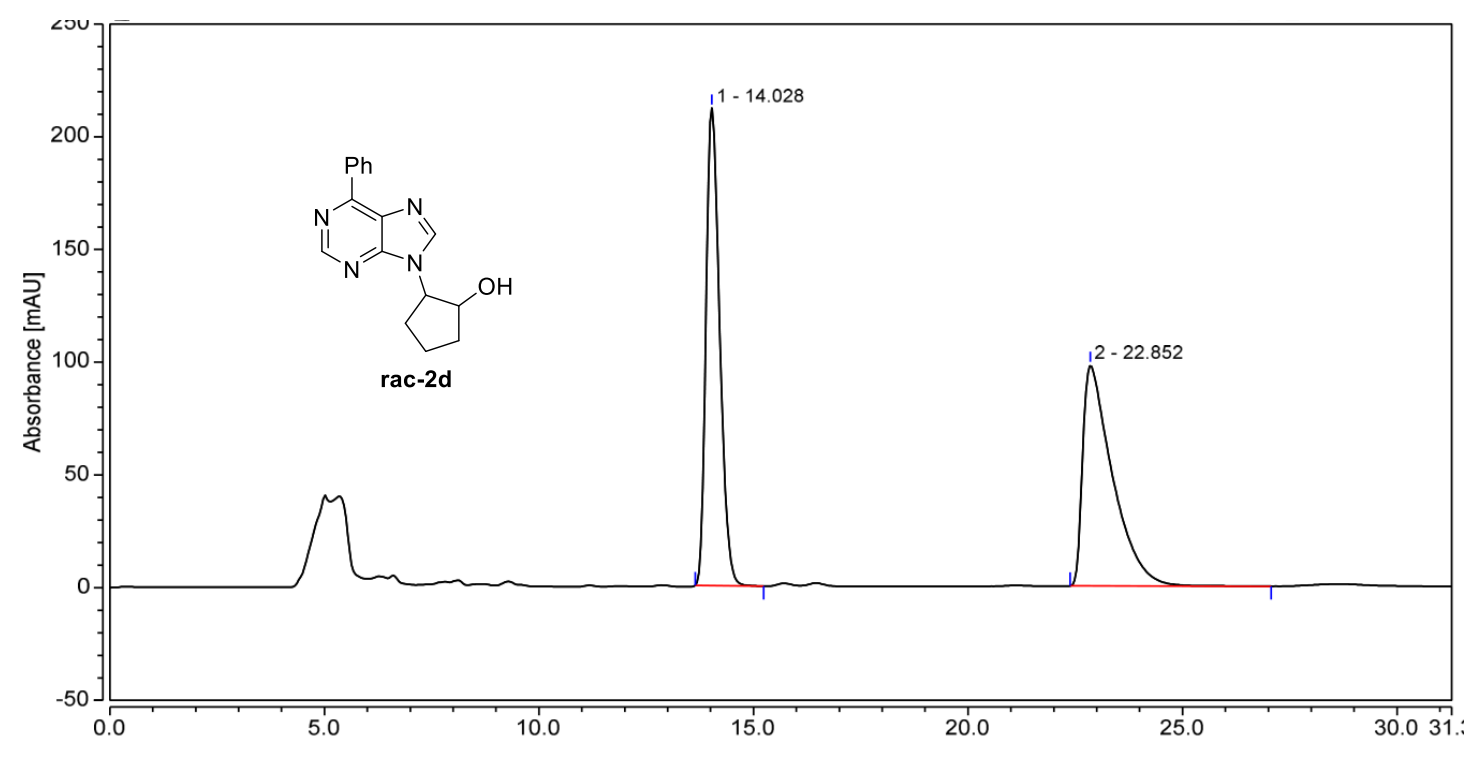

\begin{tabular}{|l|c|c|c|c|}
\hline Peak & $\begin{array}{c}\text { Retention Time } \\
\min \end{array}$ & $\begin{array}{c}\text { Area } \\
\mathrm{mAU}^{*} \min \end{array}$ & $\begin{array}{c}\text { Height } \\
\mathrm{mAU}\end{array}$ & $\begin{array}{c}\text { Area } \\
\%\end{array}$ \\
\hline 1 & 14.028 & 78.498 & 211.883 & 49.90 \\
2 & 22.852 & 78.819 & 97.846 & 50.10 \\
\hline Total & 157.317 & 309.729 & 100.00 \\
\hline
\end{tabular}

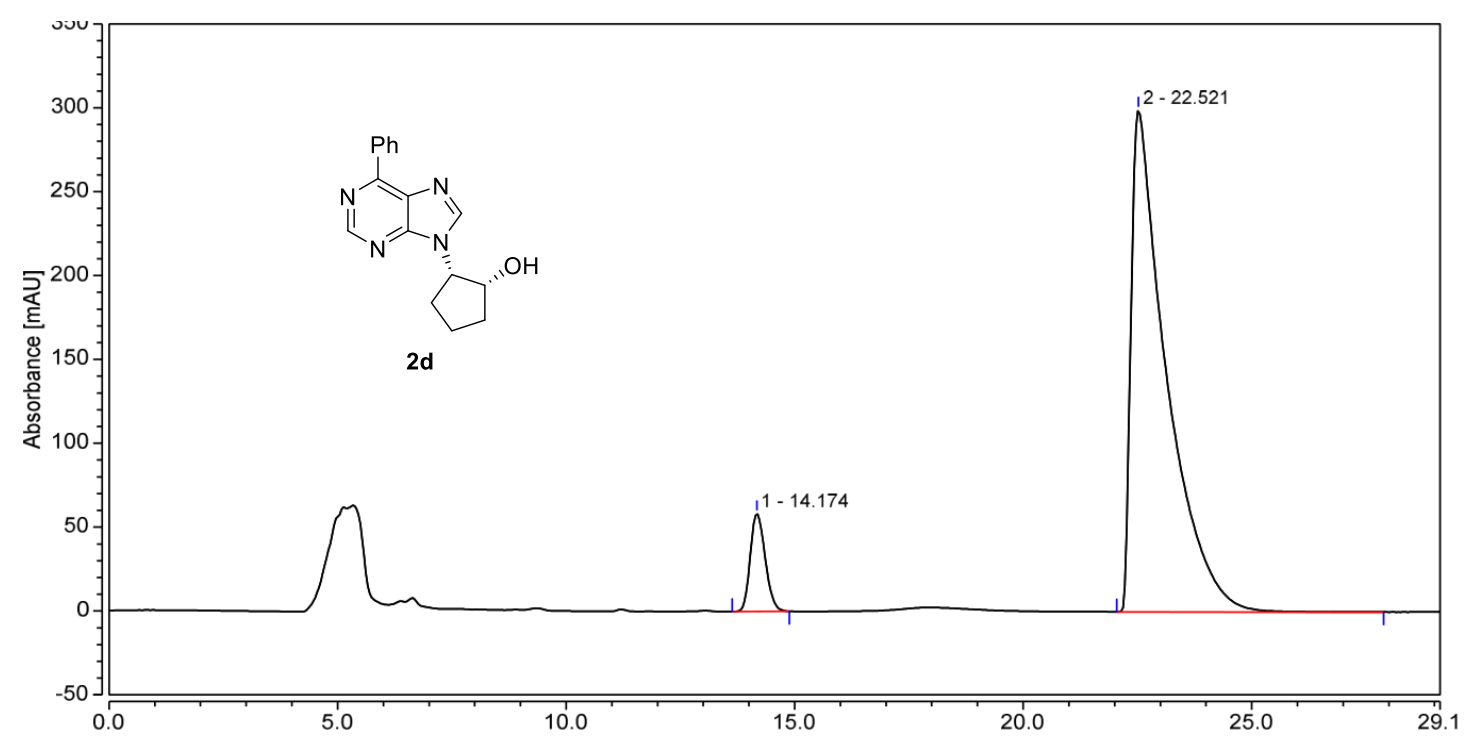

\begin{tabular}{|l|c|c|c|c|}
\hline Peak & $\begin{array}{c}\text { Retention Time } \\
\mathrm{min}\end{array}$ & $\begin{array}{c}\text { Area } \\
\mathrm{mAU}^{*} \min \end{array}$ & $\begin{array}{c}\text { Height } \\
\mathrm{mAU}\end{array}$ & $\begin{array}{c}\text { Area } \\
\%\end{array}$ \\
\hline 1 & 14.174 & 22.104 & 58.287 & 7.66 \\
2 & 22.521 & 266.621 & 299.024 & 92.34 \\
\hline \multicolumn{2}{|r|}{ Total } & $\mathbf{2 8 8 . 7 2 5}$ & $\mathbf{3 5 7 . 3 1 0}$ & $\mathbf{1 0 0 . 0 0}$
\end{tabular}




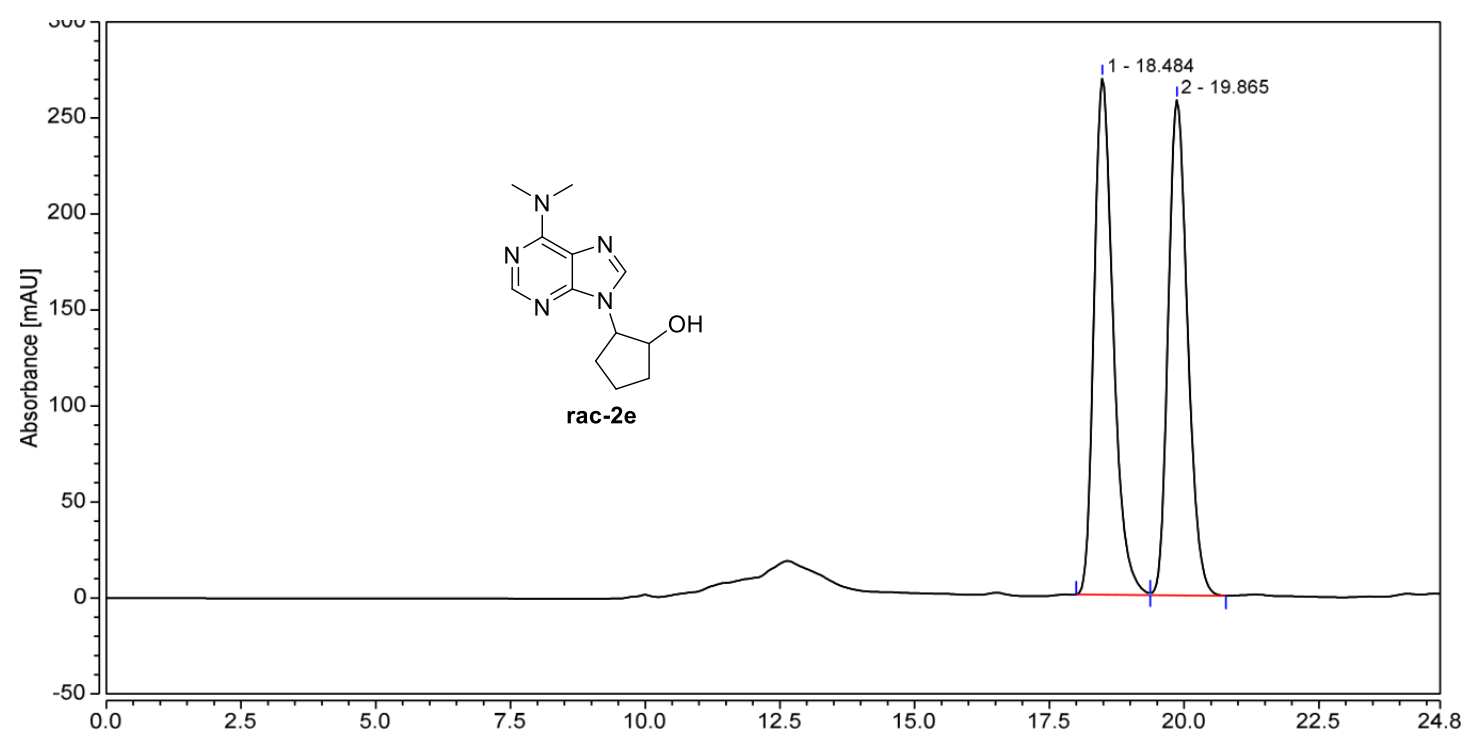

\begin{tabular}{|l|c|c|c|c|}
\hline Peak & $\begin{array}{c}\text { Retention Time } \\
\text { min }\end{array}$ & $\begin{array}{c}\text { Area } \\
\mathrm{mAU}^{*} \min \end{array}$ & $\begin{array}{c}\text { Height } \\
\mathrm{mAU}\end{array}$ & $\begin{array}{c}\text { Area } \\
\%\end{array}$ \\
\hline 1 & 18.484 & 111.248 & 269.109 & 50.80 \\
2 & 19.865 & 107.753 & 258.085 & 49.20 \\
\hline \multicolumn{2}{l|}{ Total: } & $\mathbf{2 1 9 . 0 0 1}$ & $\mathbf{5 2 7 . 1 9 3}$ & $\mathbf{1 0 0 . 0 0}$ \\
\hline
\end{tabular}

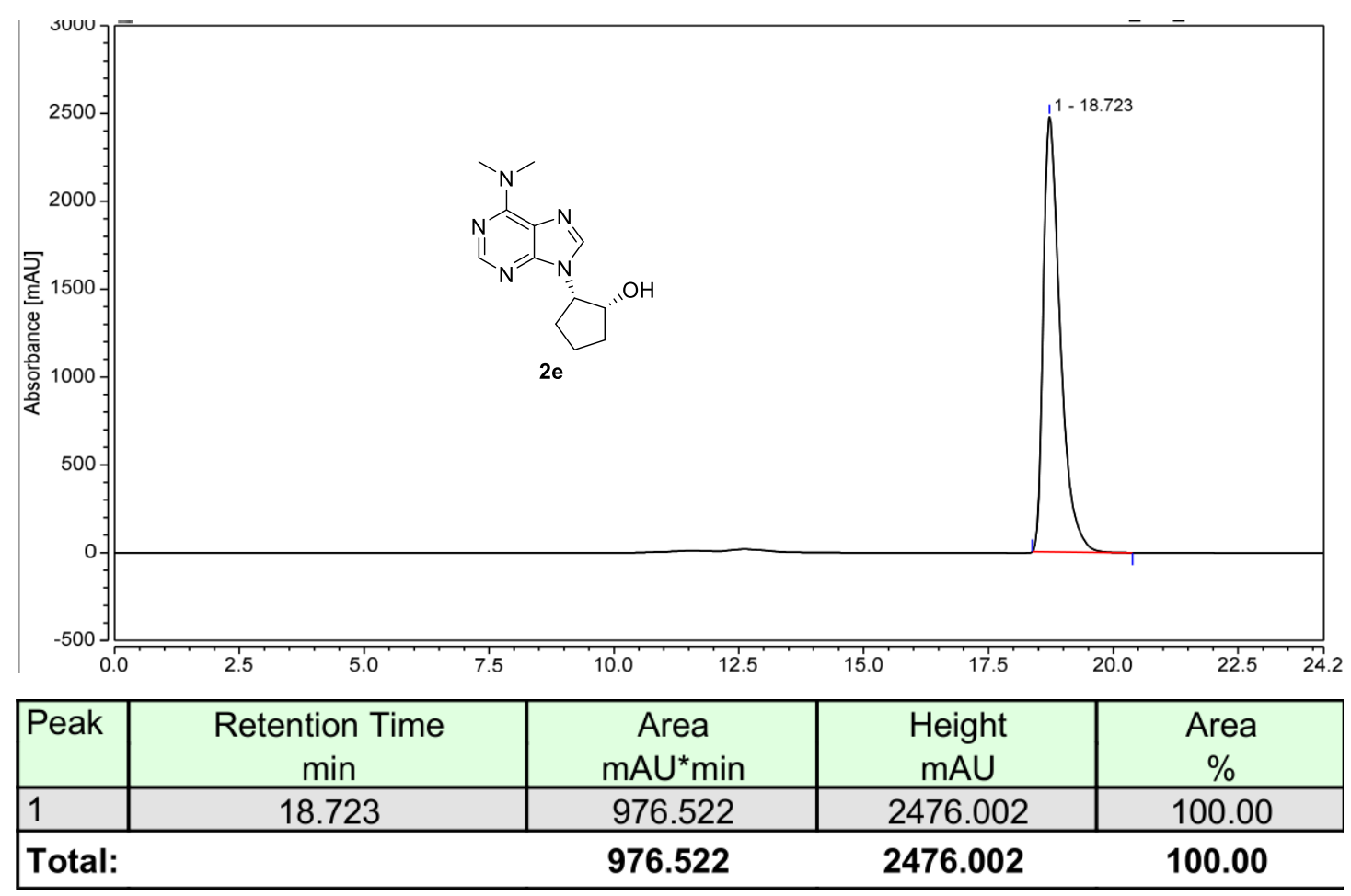




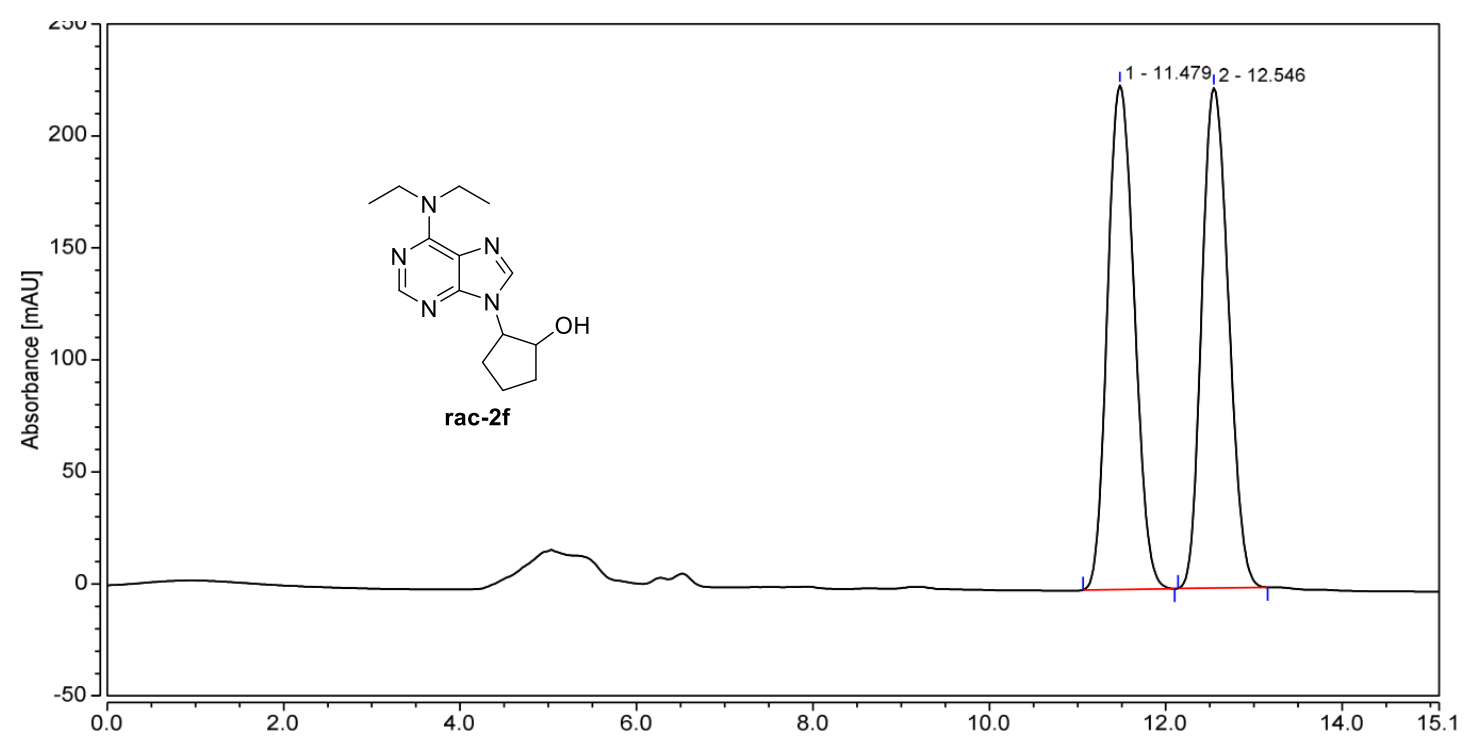

\begin{tabular}{|c|c|c|c|c|}
\hline Peak & $\begin{array}{l}\text { Retention Time } \\
\text { min }\end{array}$ & $\begin{array}{c}\text { Area } \\
m A U^{*} \min \end{array}$ & $\begin{array}{l}\text { Height } \\
\text { mAU }\end{array}$ & $\begin{array}{c}\text { Area } \\
\%\end{array}$ \\
\hline 1 & 11.479 & 79.294 & 225.139 & 50.24 \\
\hline 2 & 12.546 & 78.534 & 223.225 & 49.76 \\
\hline \multicolumn{2}{|c|}{ Total: } & 157.828 & 448.364 & 100.00 \\
\hline
\end{tabular}

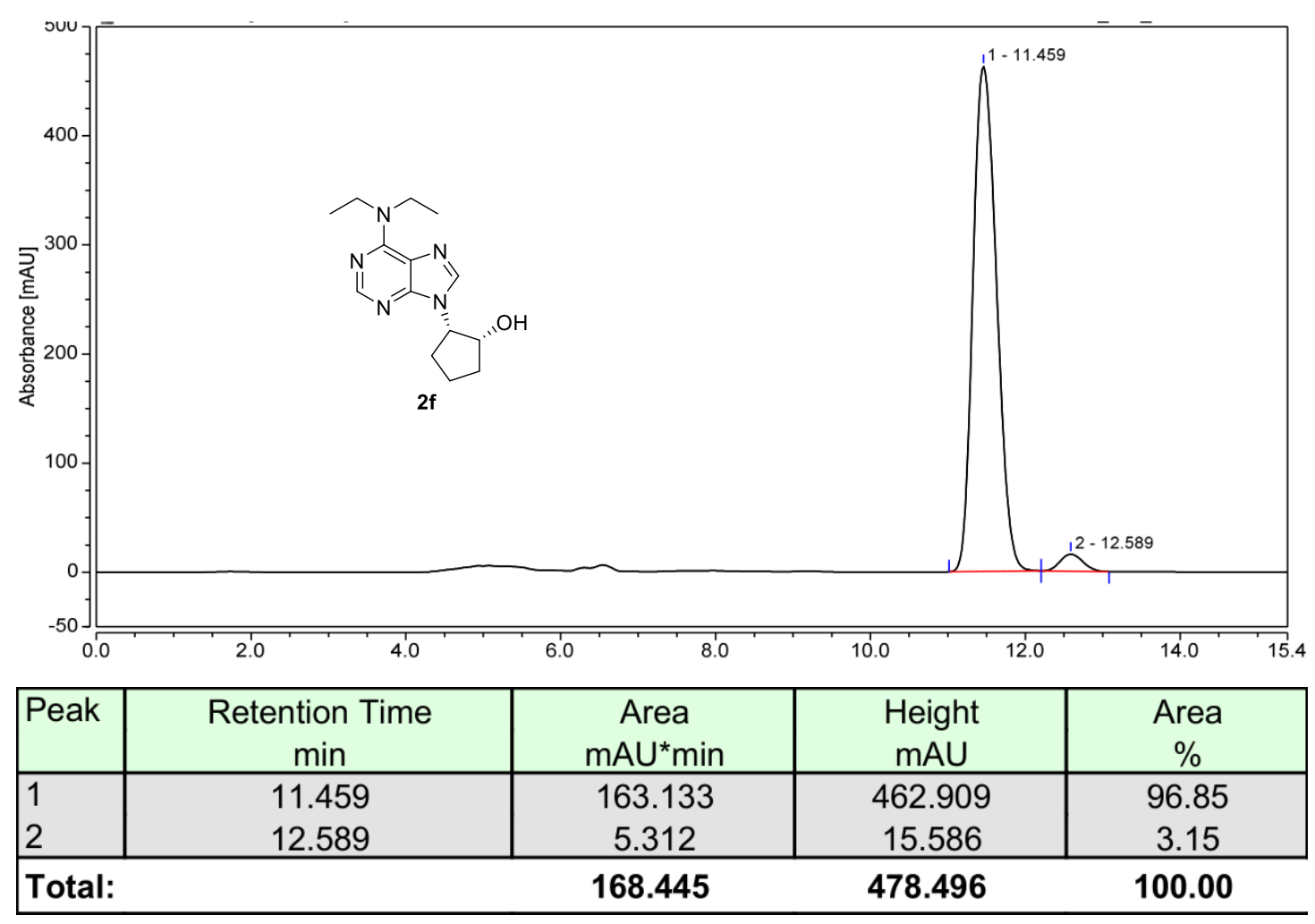




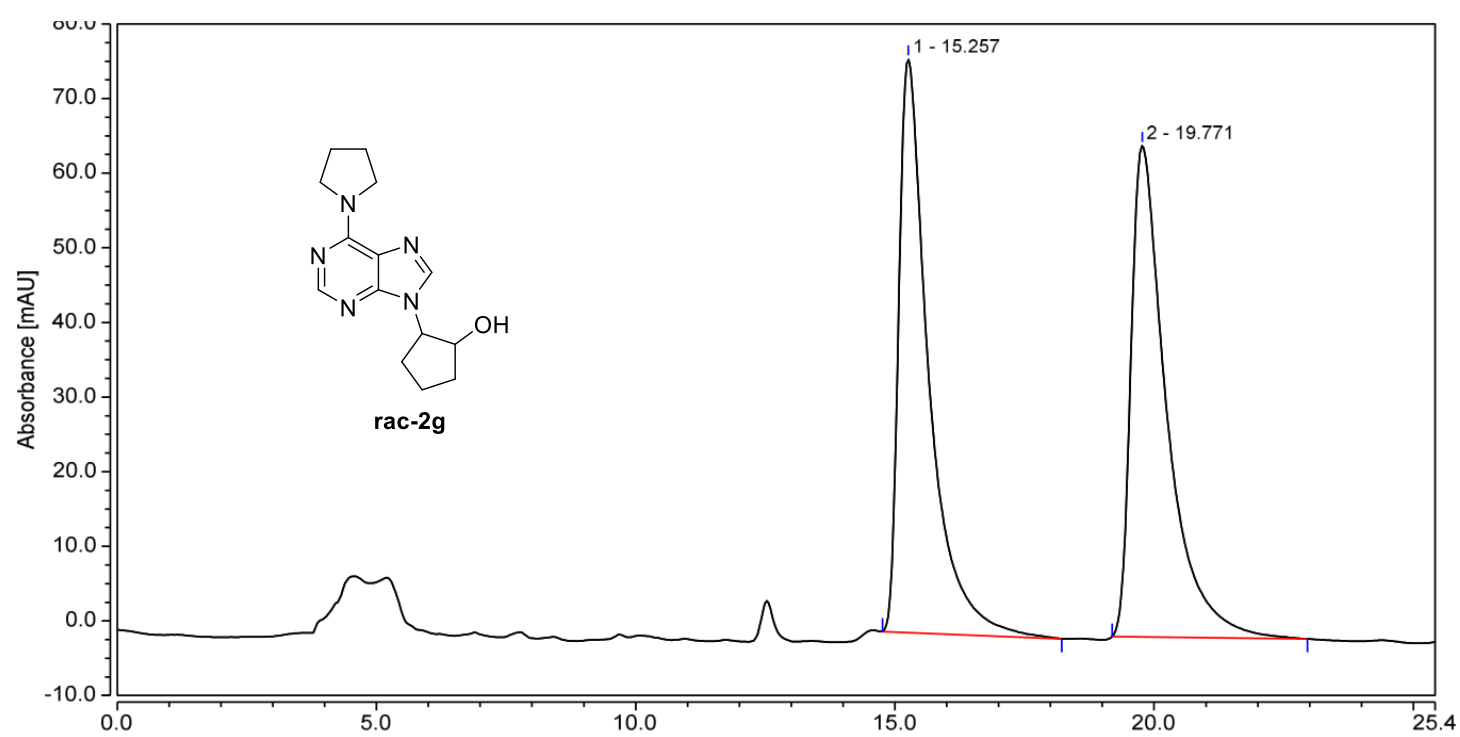

\begin{tabular}{|l|c|c|c|c|}
\hline Peak & $\begin{array}{c}\text { Retention Time } \\
\text { min }\end{array}$ & $\begin{array}{c}\text { Area } \\
\mathrm{mAU}^{*} \min \end{array}$ & $\begin{array}{c}\text { Height } \\
\mathrm{mAU}\end{array}$ & $\begin{array}{c}\text { Area } \\
\%\end{array}$ \\
\hline 1 & 15.257 & 51.597 & 76.897 & 49.55 \\
2 & 19.771 & 52.525 & 65.903 & 50.45 \\
\hline Total & $\mathbf{1 0 4 . 1 2 2}$ & $\mathbf{1 4 2 . 7 9 9}$ & $\mathbf{1 0 0 . 0 0}$ \\
\hline
\end{tabular}

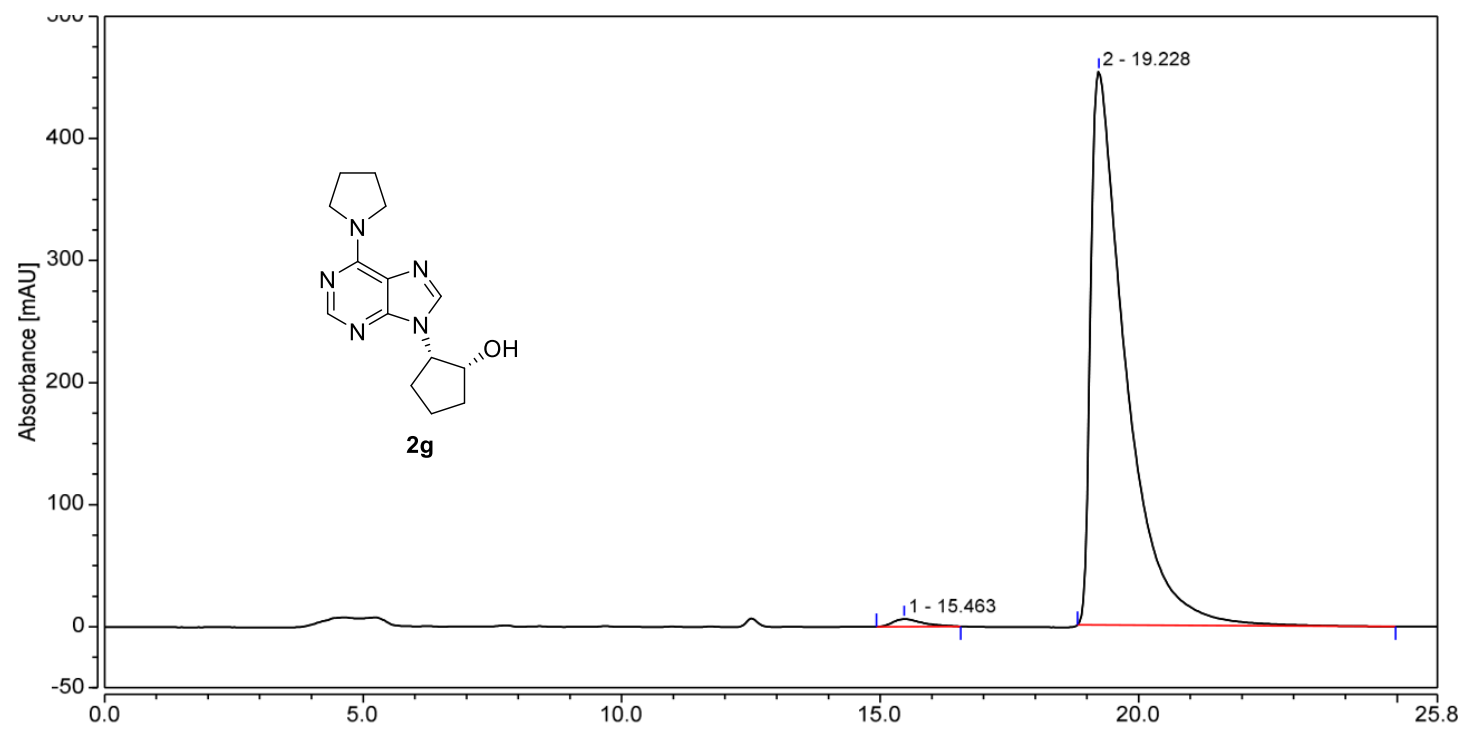

\begin{tabular}{|c|c|c|c|c|}
\hline Peak & $\begin{array}{l}\text { Retention Time } \\
\text { min }\end{array}$ & $\begin{array}{c}\text { Area } \\
m A U^{*} \min \end{array}$ & $\begin{array}{l}\text { Height } \\
\text { mAU }\end{array}$ & $\begin{array}{c}\text { Area } \\
\%\end{array}$ \\
\hline 1 & 15.463 & 4.191 & 6.253 & 1.16 \\
\hline 2 & 19.228 & 356.733 & 453.312 & 98.84 \\
\hline \multicolumn{2}{|l|}{ Total } & 360.924 & 459.564 & 100.00 \\
\hline
\end{tabular}




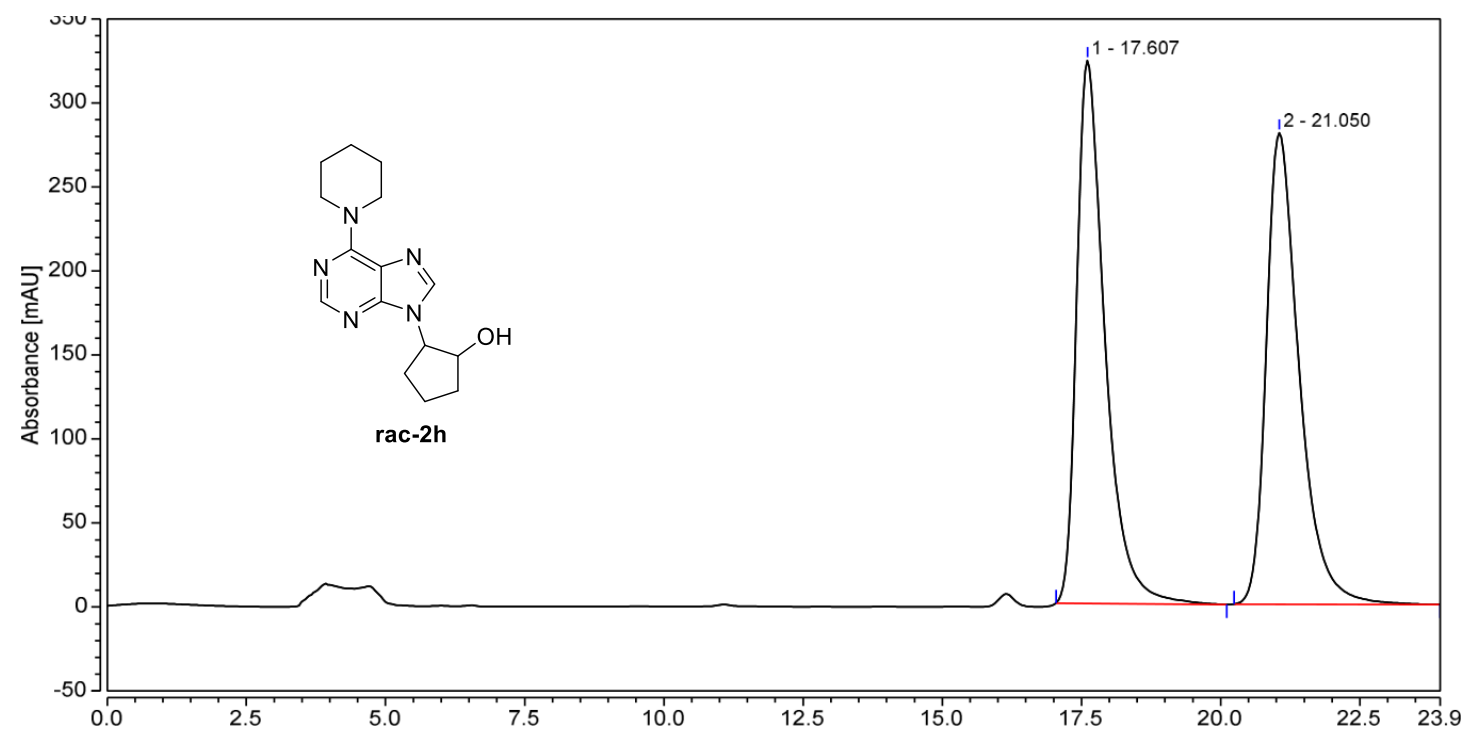

\begin{tabular}{|l|c|c|c|c|}
\hline Peak & $\begin{array}{c}\text { Retention Time } \\
\text { min }\end{array}$ & $\begin{array}{c}\text { Area } \\
\mathrm{mAU}^{*} \min \end{array}$ & $\begin{array}{c}\text { Height } \\
\mathrm{mAU}\end{array}$ & $\begin{array}{c}\text { Area } \\
\%\end{array}$ \\
\hline 1 & 17.607 & 190.796 & 323.072 & 49.75 \\
2 & 21.050 & 192.750 & 280.543 & 50.25 \\
\hline Total & $\mathbf{3 8 3 . 5 4 6}$ & $\mathbf{6 0 3 . 6 1 5}$ & $\mathbf{1 0 0 . 0 0}$ \\
\hline
\end{tabular}

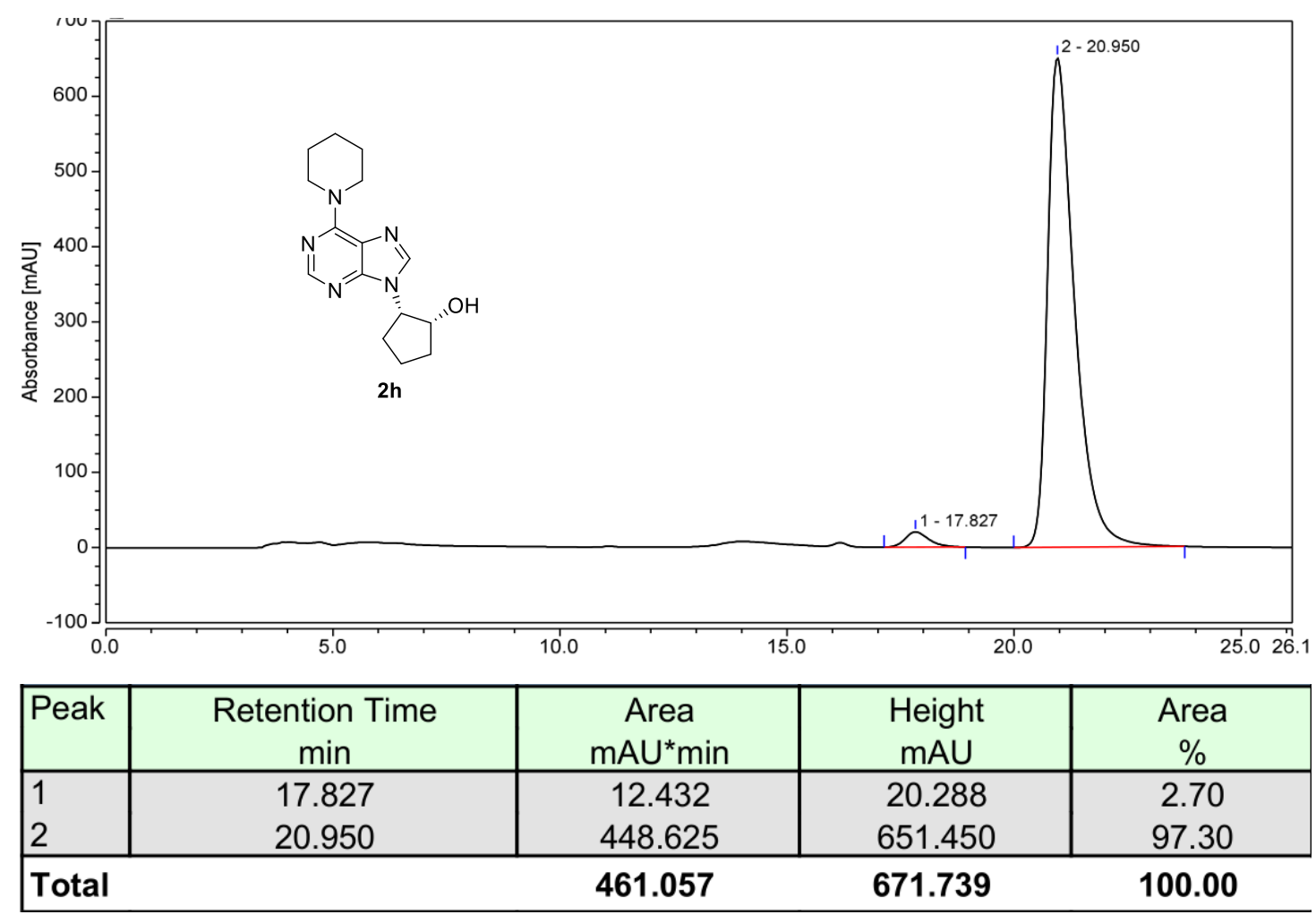




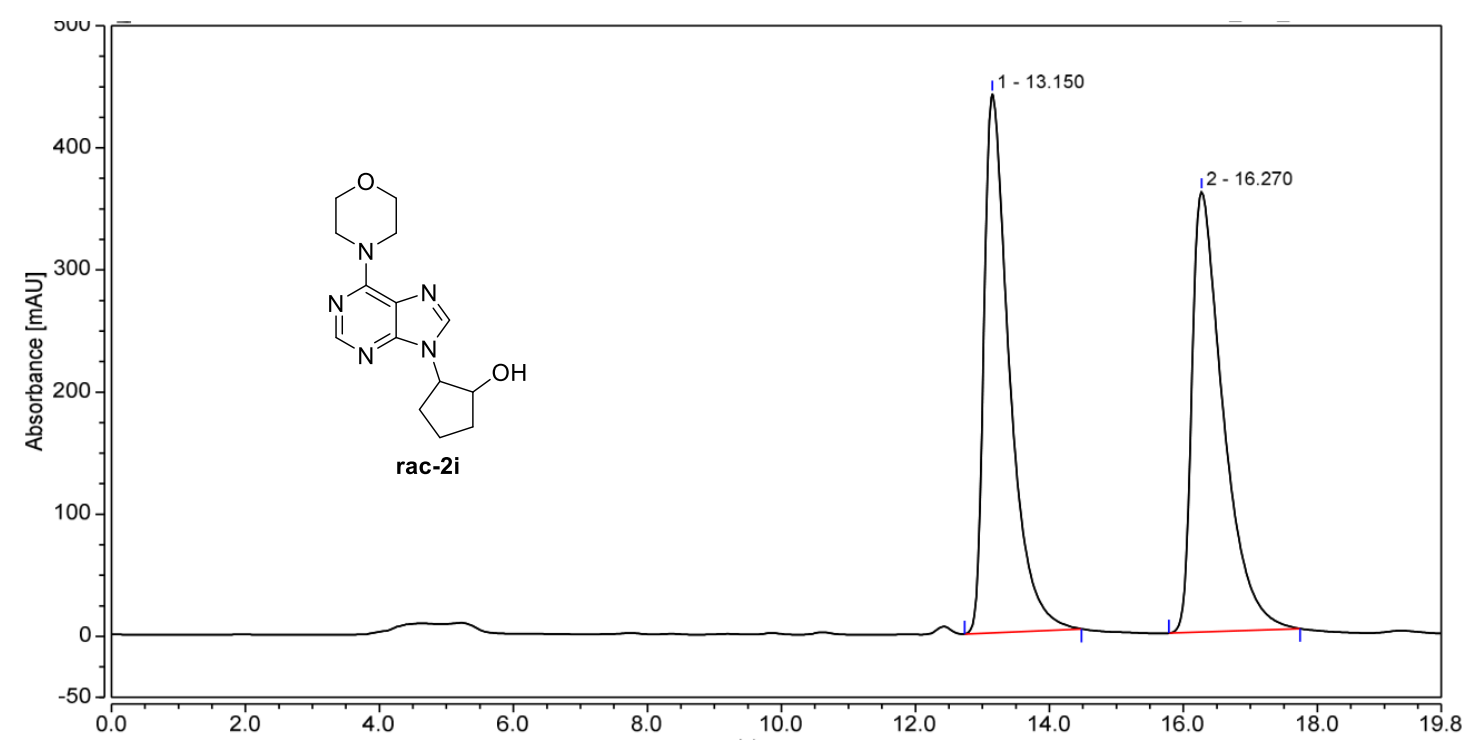

\begin{tabular}{|l|c|c|c|c|}
\hline Peak & $\begin{array}{c}\text { Retention Time } \\
\mathrm{min}\end{array}$ & $\begin{array}{c}\text { Area } \\
\mathrm{mAU}^{*} \min \end{array}$ & $\begin{array}{c}\text { Height } \\
\mathrm{mAU}\end{array}$ & $\begin{array}{c}\text { Area } \\
\%\end{array}$ \\
\hline 1 & 13.150 & 194.655 & 441.164 & 49.95 \\
2 & 16.270 & 195.052 & 360.669 & 50.05 \\
\hline \multicolumn{2}{l|}{ Total: } & $\mathbf{3 8 9 . 7 0 7}$ & $\mathbf{8 0 1 . 8 3 3}$ & $\mathbf{1 0 0 . 0 0}$ \\
\hline
\end{tabular}

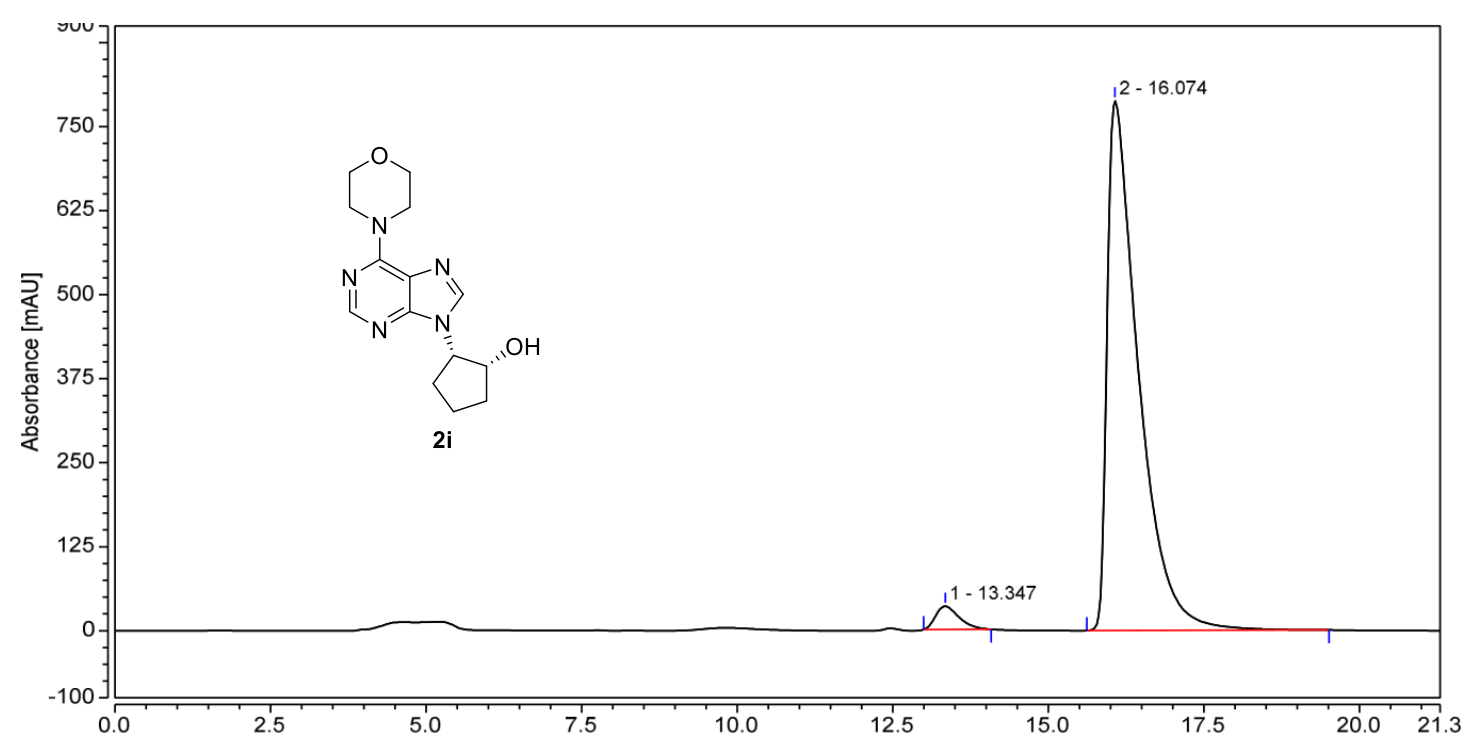

\begin{tabular}{|l|c|c|c|c|}
\hline Peak & $\begin{array}{c}\text { Retention Time } \\
\mathrm{min}\end{array}$ & $\begin{array}{c}\text { Area } \\
\mathrm{mAU}^{*} \min \end{array}$ & $\begin{array}{c}\text { Height } \\
\mathrm{mAU}\end{array}$ & $\begin{array}{c}\text { Area } \\
\%\end{array}$ \\
\hline 1 & 13.347 & 15.167 & 34.736 & 3.19 \\
2 & 16.074 & 459.905 & 788.732 & 96.81 \\
\hline \multicolumn{2}{l|}{ Total: } & $\mathbf{4 7 5 . 0 7 2}$ & $\mathbf{8 2 3 . 4 6 7}$ & $\mathbf{1 0 0 . 0 0}$ \\
\hline
\end{tabular}




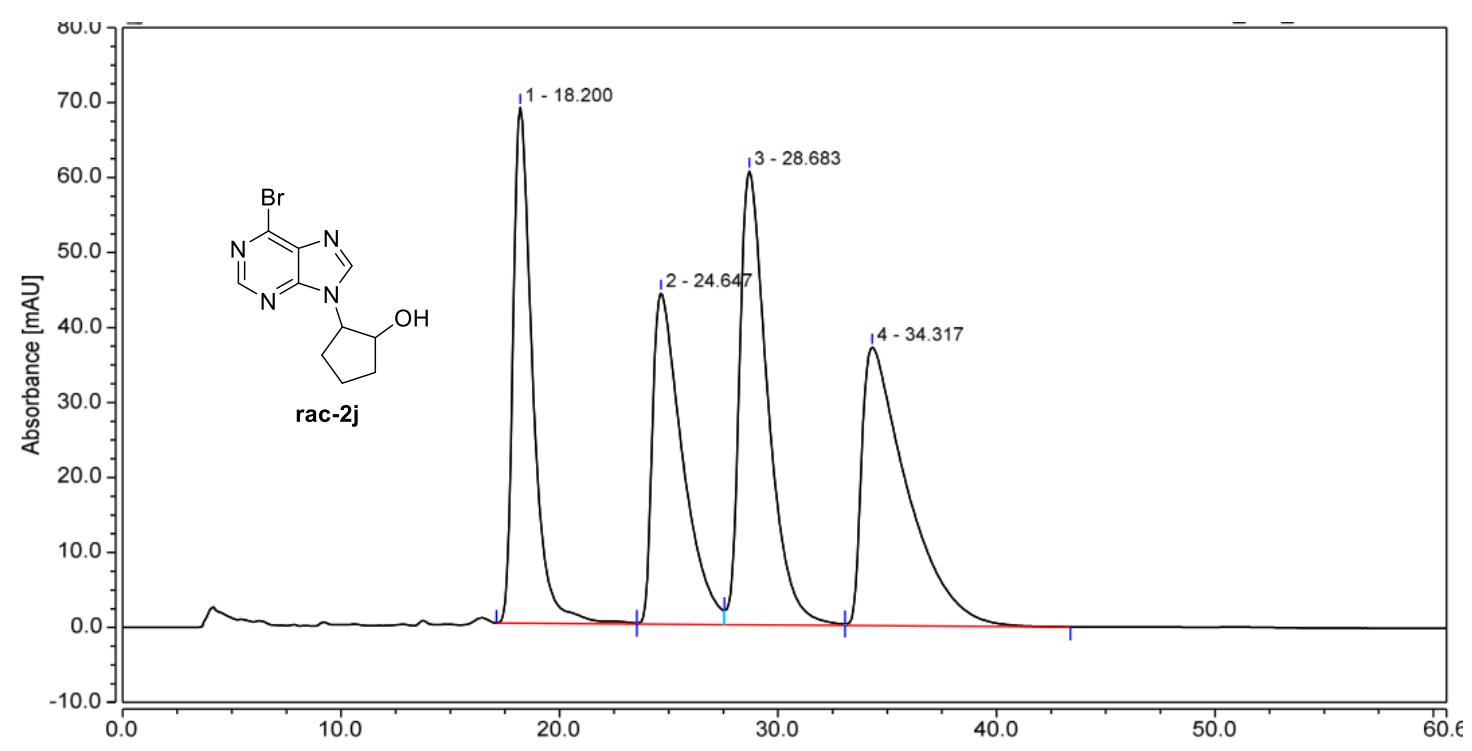

\begin{tabular}{|l|c|c|c|c|}
\hline Peak & $\begin{array}{c}\text { Retention Time } \\
\min \end{array}$ & $\begin{array}{c}\text { Area } \\
\mathrm{mAU}^{*} \min \end{array}$ & $\begin{array}{c}\text { Height } \\
\mathrm{mAU}\end{array}$ & $\begin{array}{c}\text { Area } \\
\%\end{array}$ \\
\hline 1 & 18.200 & 71.689 & 68.832 & 22.26 \\
2 & 24.647 & 70.127 & 44.177 & 21.78 \\
3 & 28.683 & 90.610 & 60.537 & 28.14 \\
4 & 34.317 & 89.595 & 37.137 & 27.82 \\
\hline Total: & $\mathbf{3 2 2 . 0 2 1}$ & $\mathbf{2 1 0 . 6 8 4}$ & $\mathbf{1 0 0 . 0 0}$ \\
\hline
\end{tabular}

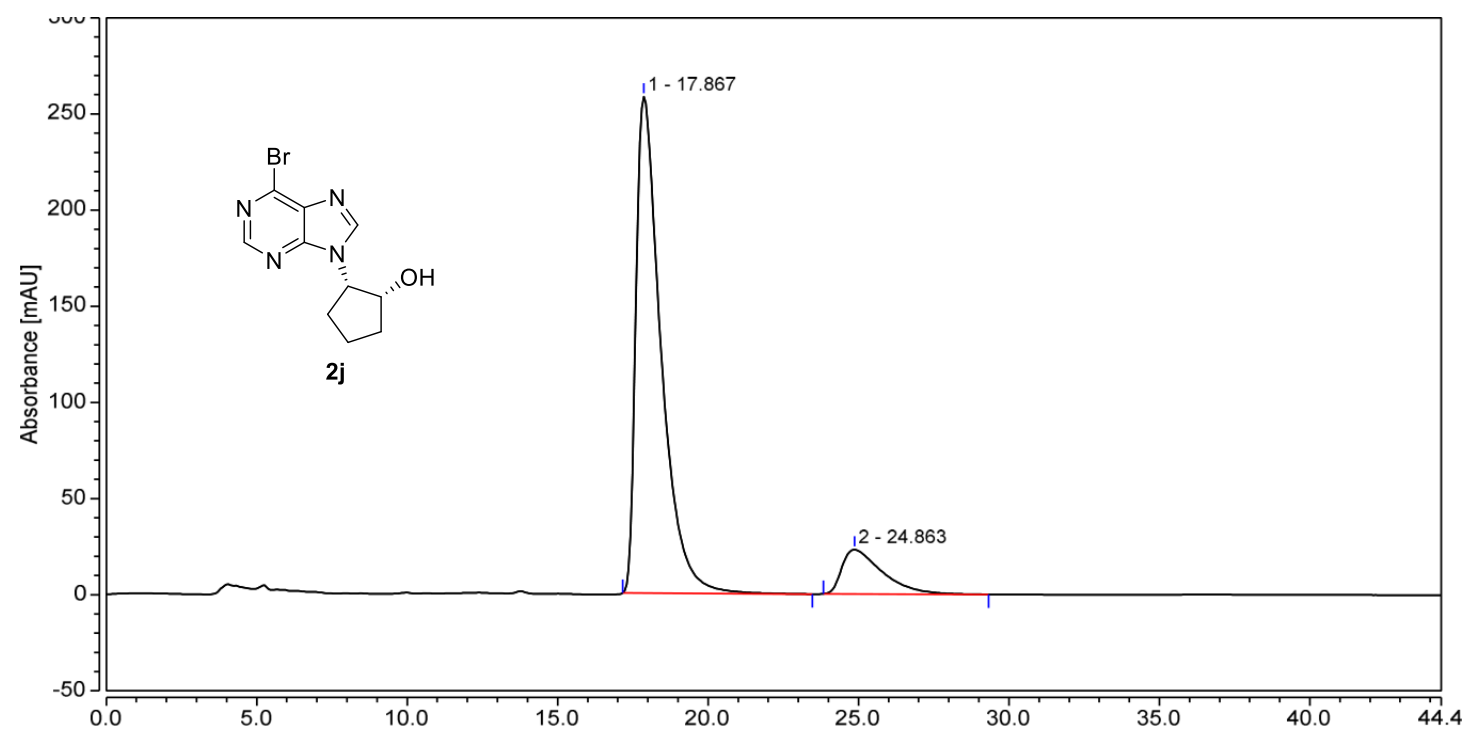

\begin{tabular}{|c|c|c|c|c|}
\hline Peak & $\begin{array}{c}\text { Retention Time } \\
\text { min }\end{array}$ & $\begin{array}{c}\text { Area } \\
\text { mAU*min }\end{array}$ & $\begin{array}{l}\text { Height } \\
\text { mAU }\end{array}$ & $\begin{array}{c}\text { Area } \\
\%\end{array}$ \\
\hline 1 & 17.867 & 249.091 & 258.100 & 87.41 \\
\hline 2 & 24.863 & 35.878 & 23.064 & 12.59 \\
\hline \multicolumn{2}{|l|}{ Total: } & 284.969 & 281.165 & 100.00 \\
\hline
\end{tabular}




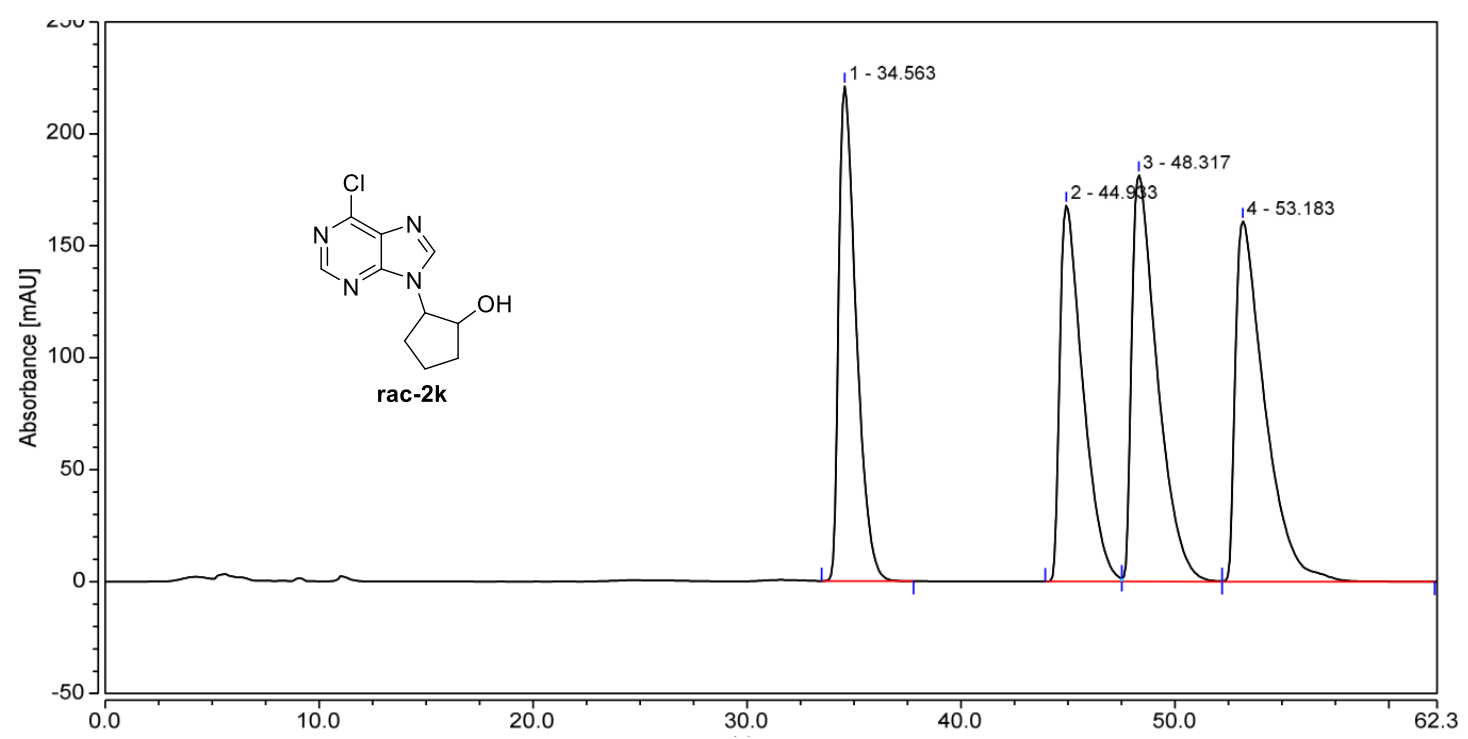

\begin{tabular}{|l|c|c|c|c|}
\hline Peak & $\begin{array}{c}\text { Retention Time } \\
\text { min }\end{array}$ & $\begin{array}{c}\text { Area } \\
\mathrm{mAU}^{*} \min \end{array}$ & $\begin{array}{c}\text { Height } \\
\mathrm{mAU}\end{array}$ & $\begin{array}{c}\text { Area } \\
\%\end{array}$ \\
\hline 1 & 34.563 & 209.673 & 221.043 & 22.61 \\
2 & 44.933 & 209.959 & 168.095 & 22.64 \\
3 & 48.317 & 252.304 & 181.716 & 27.21 \\
4 & 53.183 & 255.471 & 161.003 & 27.55 \\
\hline Total: & $\mathbf{9 2 7 . 4 0 7}$ \\
\hline
\end{tabular}

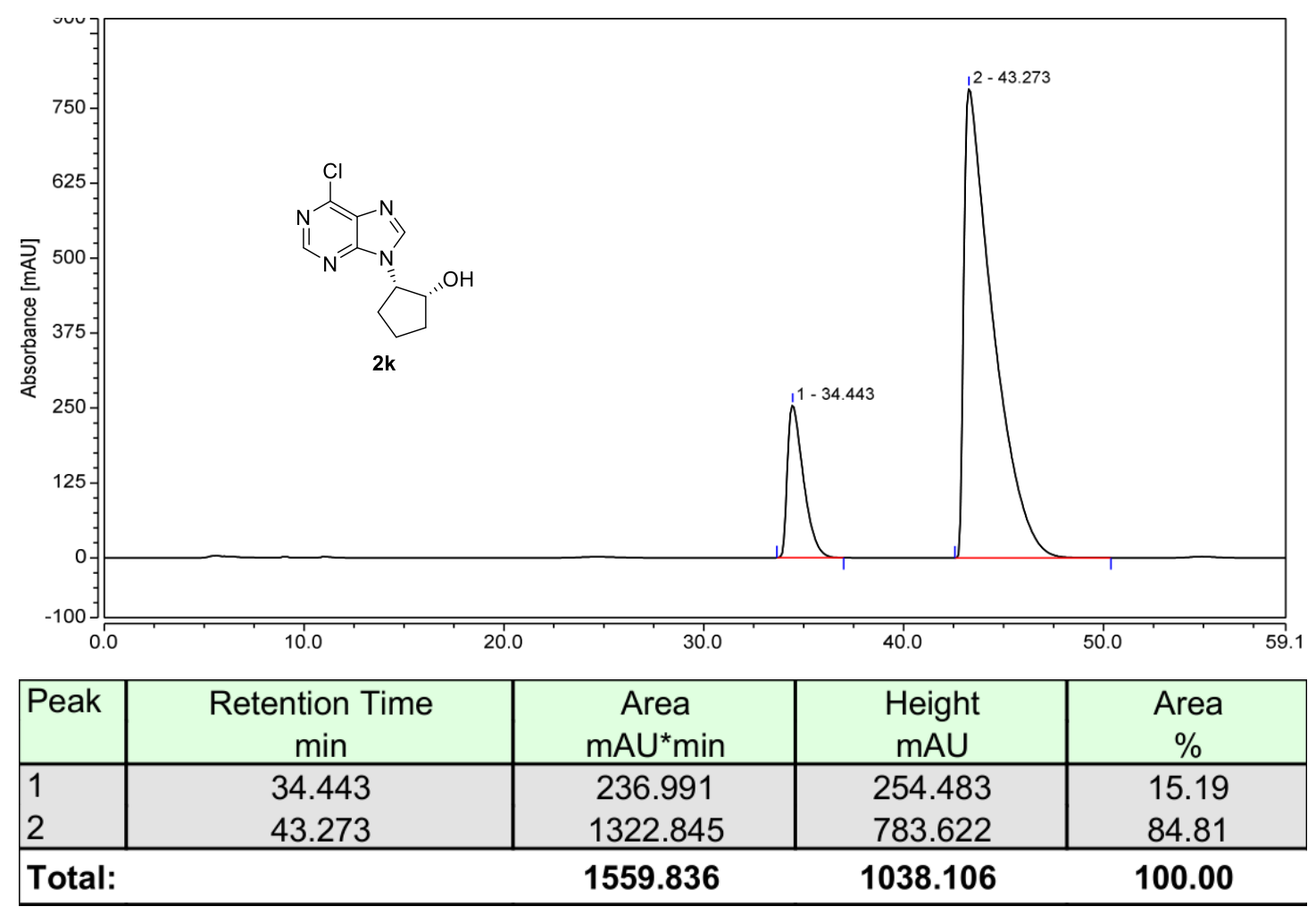




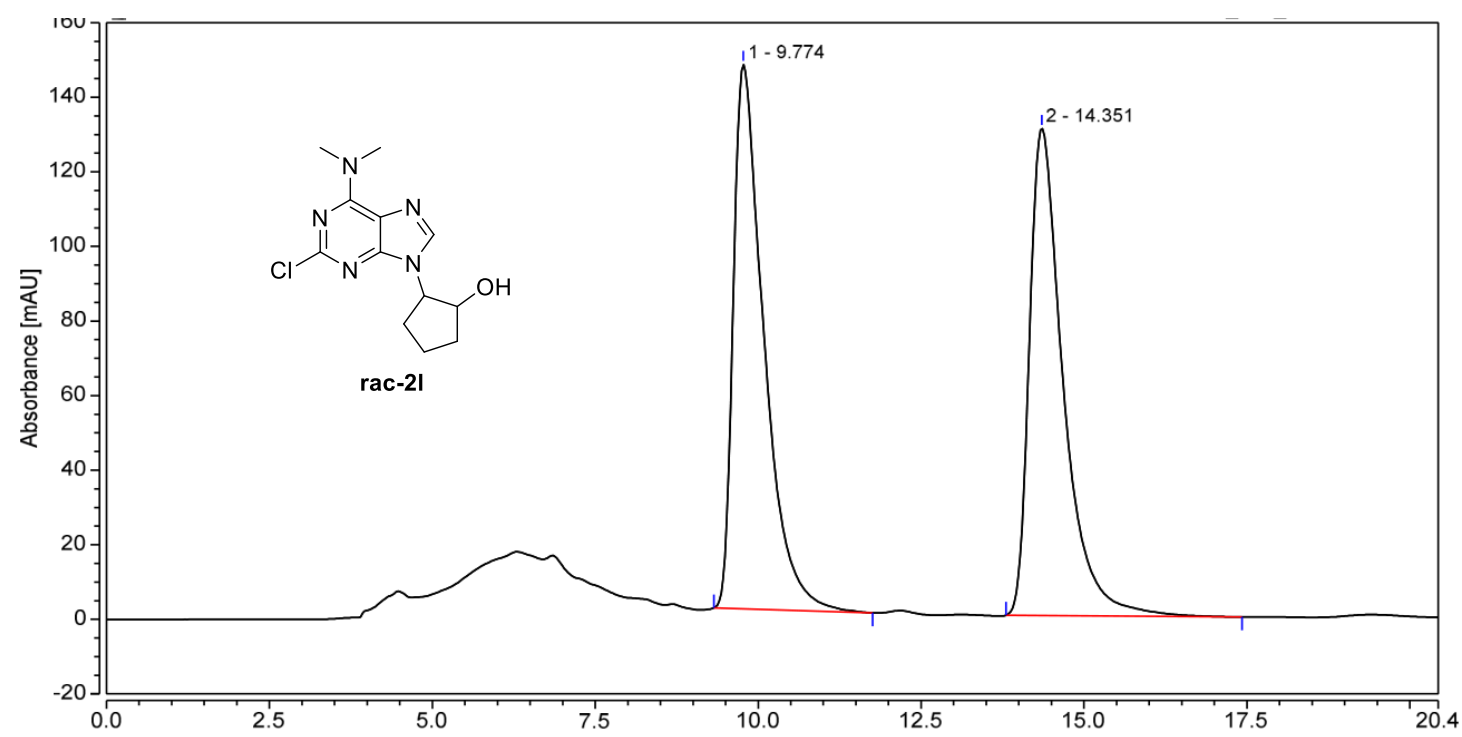

\begin{tabular}{|l|c|c|c|c|}
\hline Peak & $\begin{array}{c}\text { Retention Time } \\
\min \end{array}$ & $\begin{array}{c}\text { Area } \\
\mathrm{mAU}^{*} \min \end{array}$ & $\begin{array}{c}\text { Height } \\
\mathrm{mAU}\end{array}$ & $\begin{array}{c}\text { Area } \\
\%\end{array}$ \\
\hline 1 & 9.774 & 79.603 & 146.036 & 50.31 \\
2 & 14.351 & 78.627 & 130.704 & 49.69 \\
\hline Total & \multicolumn{1}{|c|}{$\mathbf{1 5 8 . 2 3 0}$} & $\mathbf{2 7 6 . 7 4 0}$ & $\mathbf{1 0 0 . 0 0}$ \\
\hline
\end{tabular}

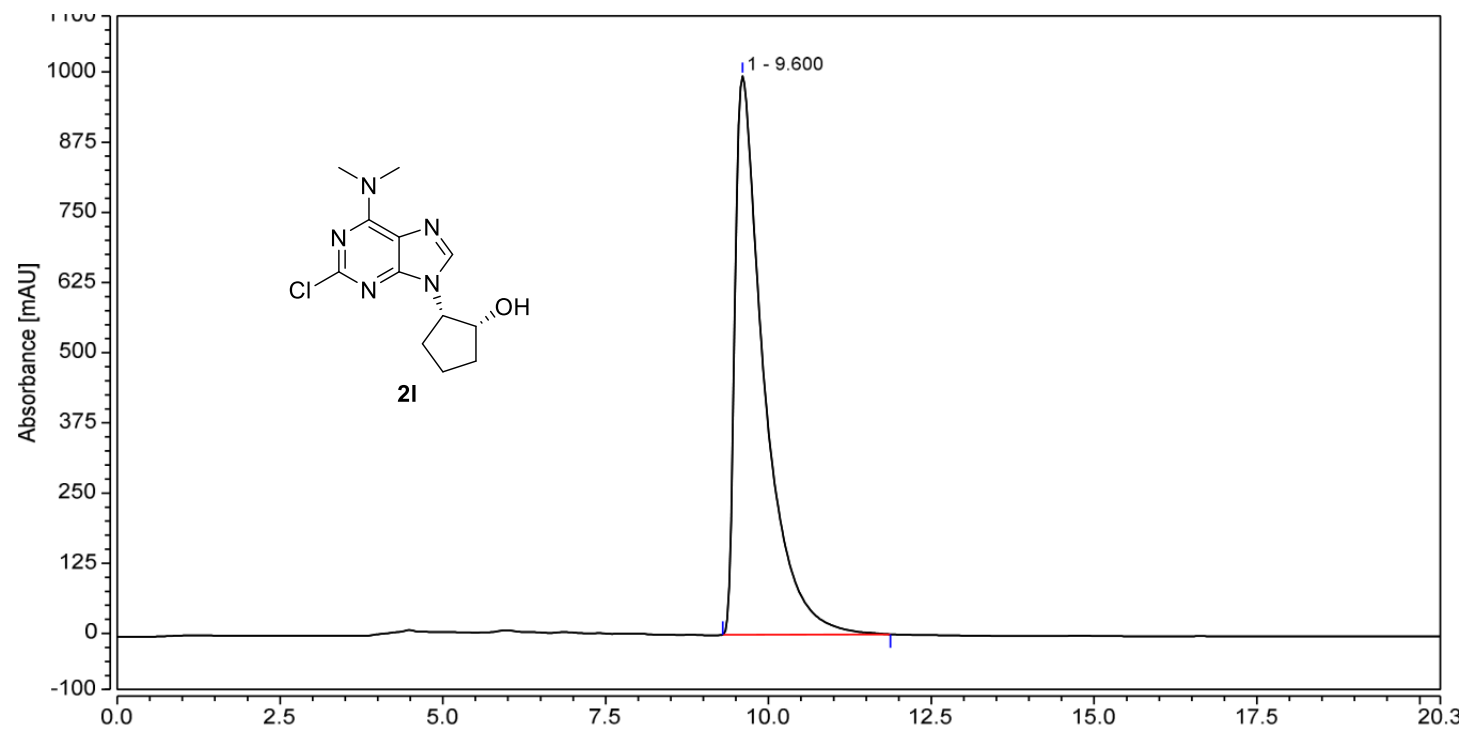

\begin{tabular}{|l|c|c|c|c|}
\hline Peak & $\begin{array}{c}\text { Retention Time } \\
\text { min }\end{array}$ & $\begin{array}{c}\text { Area } \\
\mathrm{mAU}^{*} \text { min }\end{array}$ & $\begin{array}{c}\text { Height } \\
\mathrm{mAU}\end{array}$ & $\begin{array}{c}\text { Area } \\
\%\end{array}$ \\
\hline 1 & 9.600 & 516.249 & 994.792 & 100.00 \\
\hline Total & $\mathbf{5 1 6 . 2 4 9}$ & $\mathbf{9 9 4 . 7 9 2}$ & $\mathbf{1 0 0 . 0 0}$ \\
\hline
\end{tabular}




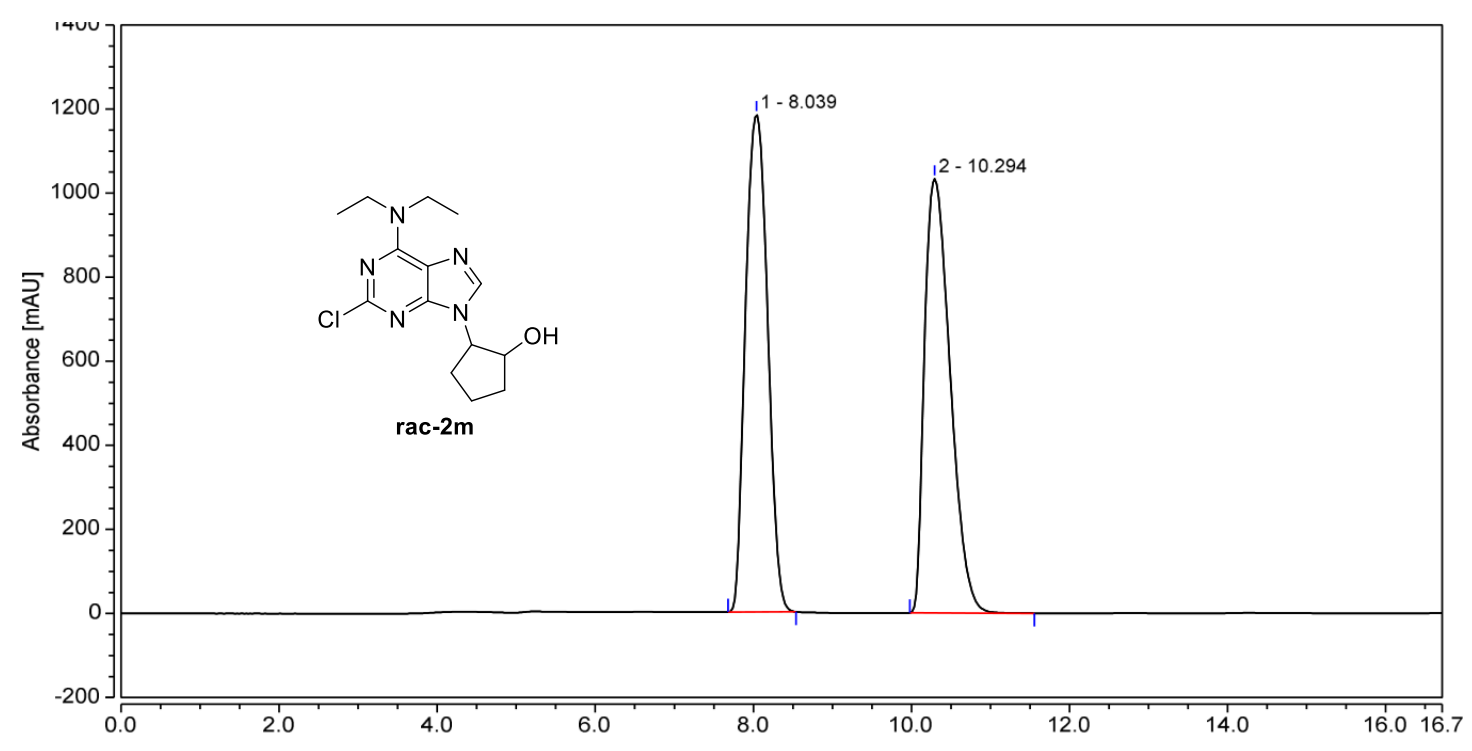

\begin{tabular}{|l|c|c|c|c|}
\hline Peak & $\begin{array}{c}\text { Retention Time } \\
\text { min }\end{array}$ & $\begin{array}{c}\text { Area } \\
\mathrm{mAU}^{*} \min \end{array}$ & $\begin{array}{c}\text { Height } \\
\mathrm{mAU}\end{array}$ & $\begin{array}{c}\text { Area } \\
\%\end{array}$ \\
\hline 1 & 8.039 & 383.994 & 1183.605 & 49.73 \\
2 & 10.294 & 388.163 & 1032.735 & 50.27 \\
\hline \multicolumn{2}{l|}{ Total: } & $\mathbf{7 7 2 . 1 5 7}$ & $\mathbf{2 2 1 6 . 3 4 0}$ & $\mathbf{1 0 0 . 0 0}$ \\
\hline
\end{tabular}

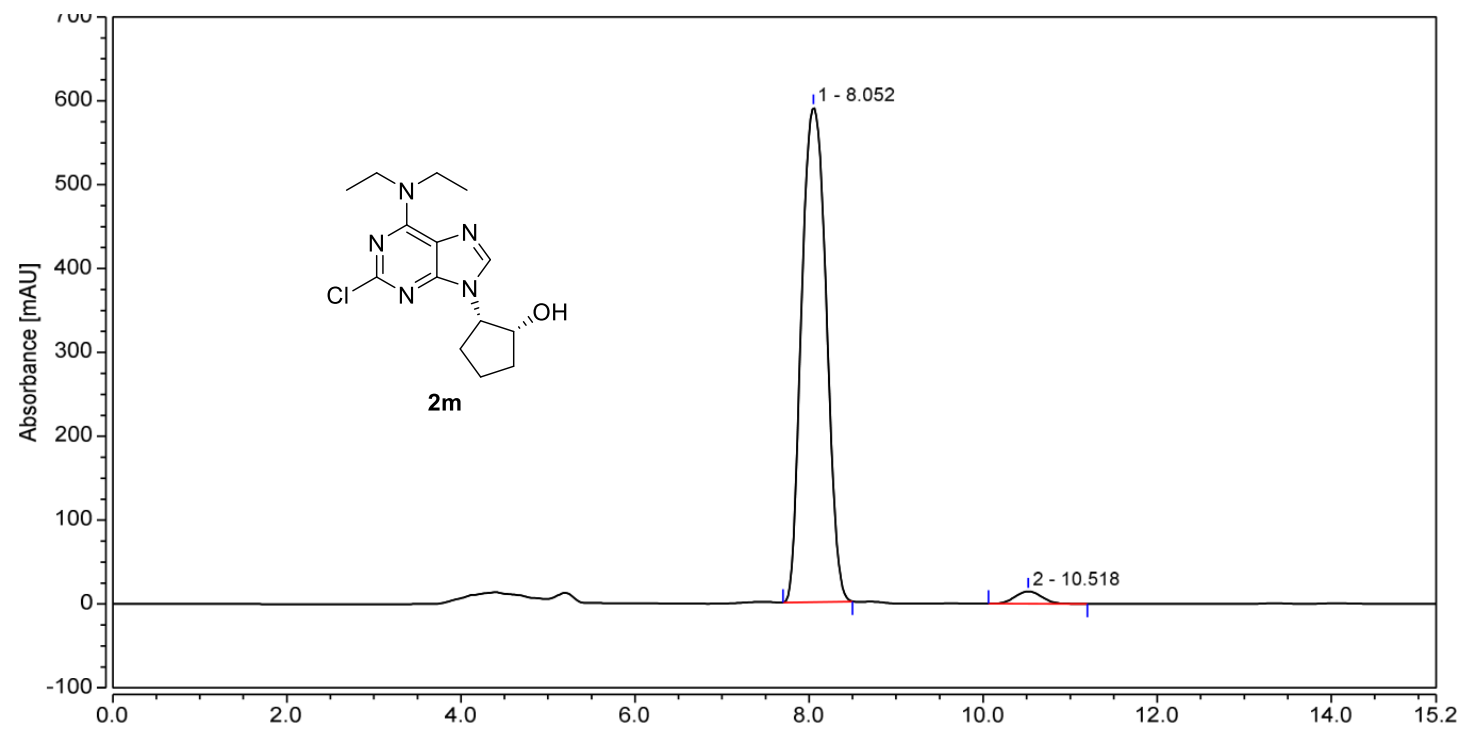

\begin{tabular}{|c|c|c|c|c|}
\hline Peak & $\begin{array}{c}\text { Retention Time } \\
\text { min }\end{array}$ & $\begin{array}{c}\text { Area } \\
m A U^{*} \min \end{array}$ & $\begin{array}{l}\text { Height } \\
\text { mAU }\end{array}$ & $\begin{array}{c}\text { Area } \\
\%\end{array}$ \\
\hline $\begin{array}{l}1 \\
2\end{array}$ & $\begin{array}{c}8.052 \\
10.518 \\
\end{array}$ & $\begin{array}{c}193.396 \\
5.265 \\
\end{array}$ & $\begin{array}{c}589.477 \\
14.793 \\
\end{array}$ & $\begin{array}{l}97.35 \\
2.65 \\
\end{array}$ \\
\hline \multicolumn{2}{|c|}{ Total: } & 198.661 & 604.271 & 100.00 \\
\hline
\end{tabular}




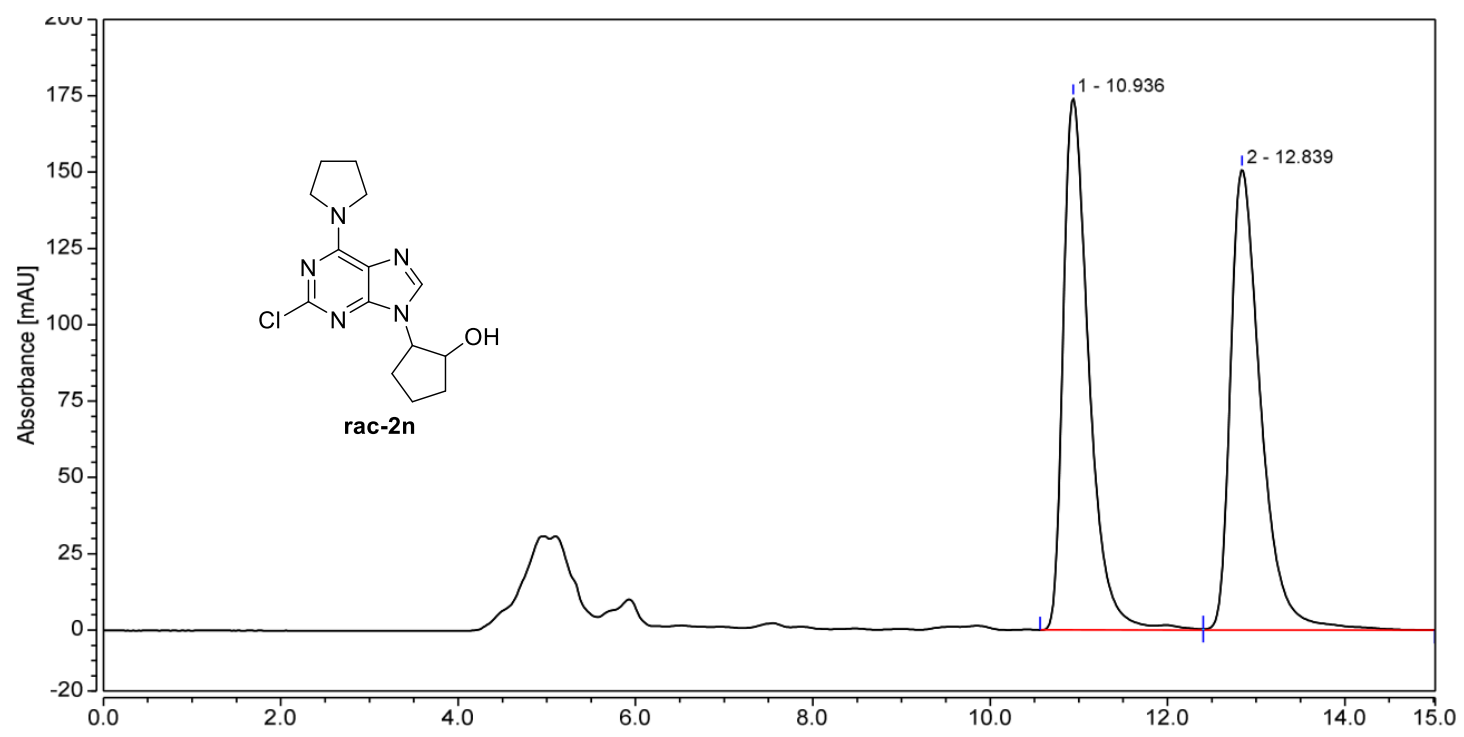

\begin{tabular}{|l|c|c|c|c|}
\hline Peak & $\begin{array}{c}\text { Retention Time } \\
\mathrm{min}\end{array}$ & $\begin{array}{c}\text { Area } \\
\mathrm{mAU}^{*} \min \end{array}$ & $\begin{array}{c}\text { Height } \\
\mathrm{mAU}\end{array}$ & $\begin{array}{c}\text { Area } \\
\%\end{array}$ \\
\hline 1 & 10.936 & 58.729 & 174.363 & 49.54 \\
2 & 12.839 & 59.810 & 150.997 & 50.46 \\
\hline Total & $\mathbf{1 1 8 . 5 3 9}$ & $\mathbf{3 2 5 . 3 6 0}$ & $\mathbf{1 0 0 . 0 0}$ \\
\hline
\end{tabular}

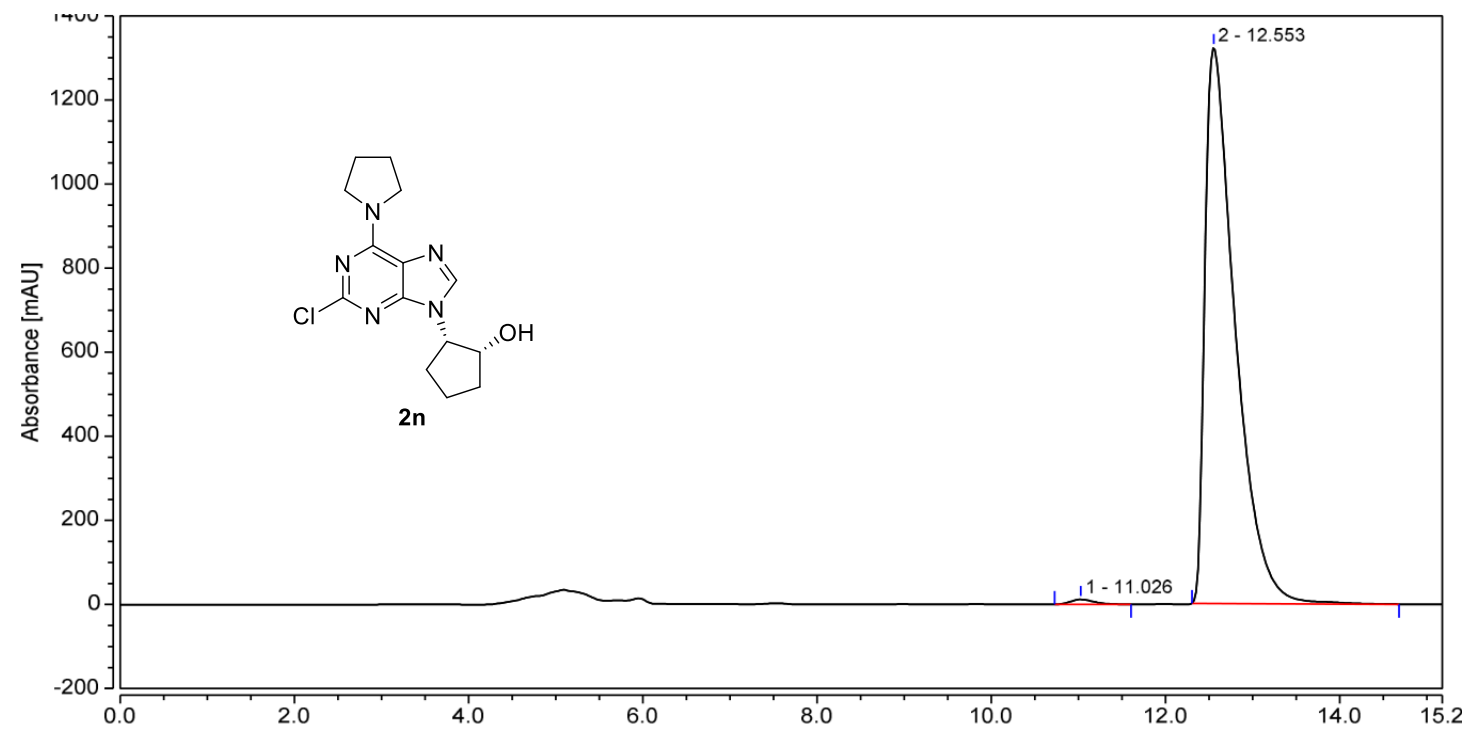

\begin{tabular}{|l|c|c|c|c|}
\hline Peak & $\begin{array}{c}\text { Retention Time } \\
\mathrm{min}\end{array}$ & $\begin{array}{c}\text { Area } \\
\mathrm{mAU}^{*} \min \end{array}$ & $\begin{array}{c}\text { Height } \\
\mathrm{mAU}\end{array}$ & $\begin{array}{c}\text { Area } \\
\%\end{array}$ \\
\hline 1 & 11.026 & 3.740 & 11.815 & 0.70 \\
2 & 12.553 & 532.063 & 1322.830 & 99.30 \\
\hline \multicolumn{2}{|r}{ Total } & $\mathbf{5 3 5 . 8 0 3}$ & $\mathbf{1 3 3 4 . 6 4 4}$ & $\mathbf{1 0 0 . 0 0}$ \\
\hline
\end{tabular}




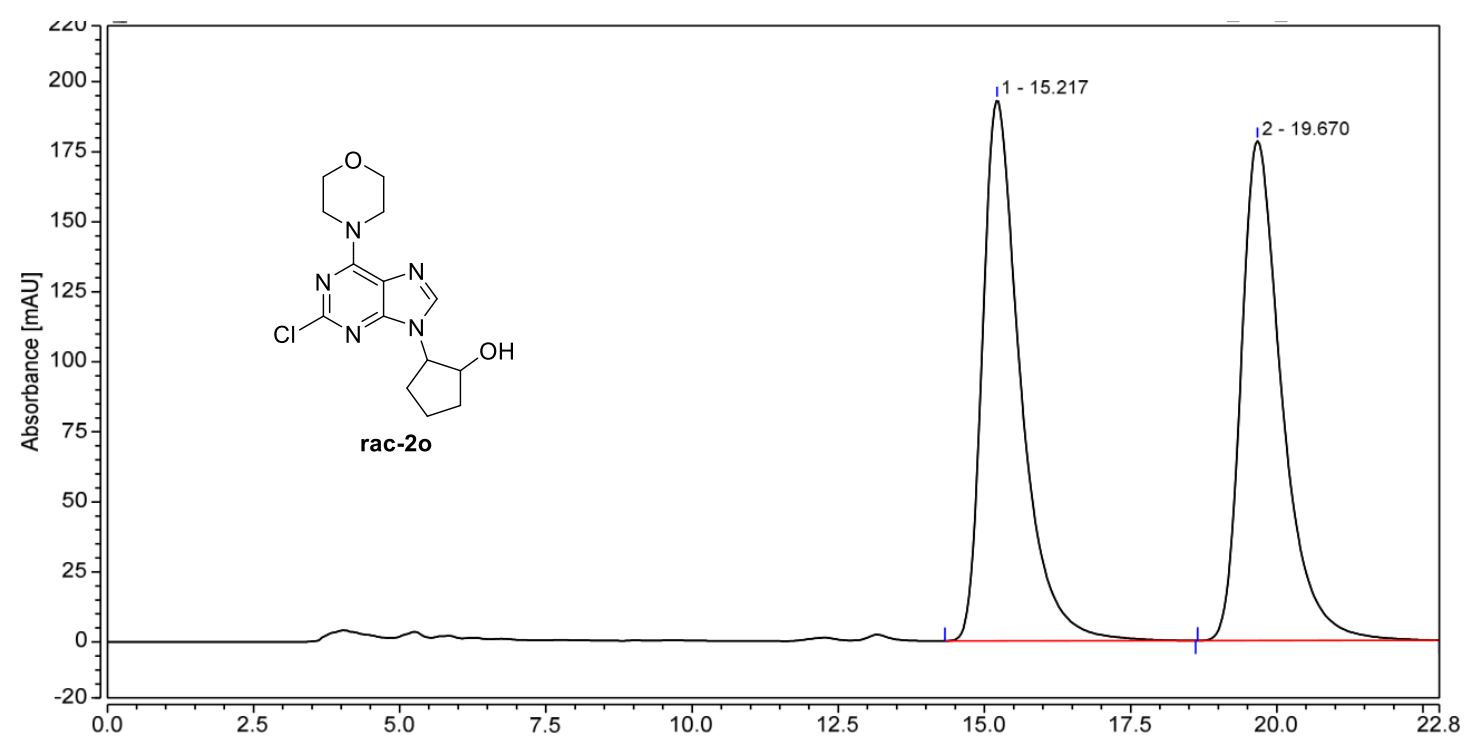

\begin{tabular}{|l|c|c|c|c|}
\hline Peak & $\begin{array}{c}\text { Retention Time } \\
\text { min }\end{array}$ & $\begin{array}{c}\text { Area } \\
\mathrm{mAU}^{*} \min \end{array}$ & $\begin{array}{c}\text { Height } \\
\mathrm{mAU}\end{array}$ & $\begin{array}{c}\text { Area } \\
\%\end{array}$ \\
\hline 1 & 15.217 & 145.631 & 193.114 & 50.23 \\
2 & 19.670 & 144.270 & 178.450 & 49.77 \\
\hline \multicolumn{2}{|l}{ Total: } & $\mathbf{2 8 9 . 9 0 1}$ & $\mathbf{3 7 1 . 5 6 4}$ & $\mathbf{1 0 0 . 0 0}$ \\
\hline
\end{tabular}

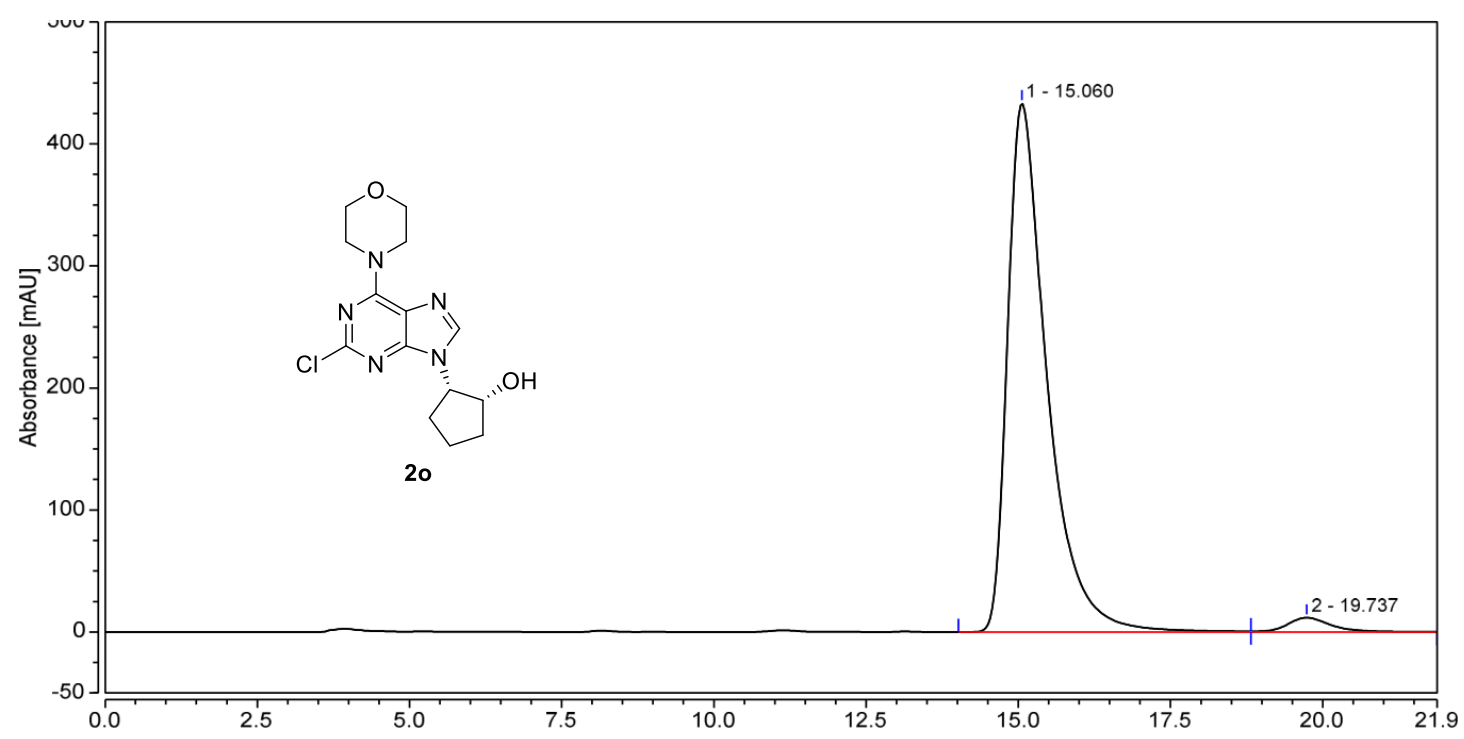

\begin{tabular}{|l|c|c|c|c|}
\hline Peak & $\begin{array}{c}\text { Retention Time } \\
\mathrm{min}\end{array}$ & $\begin{array}{c}\text { Area } \\
\mathrm{mAU}^{*} \min \end{array}$ & $\begin{array}{c}\text { Height } \\
\mathrm{mAU}\end{array}$ & $\begin{array}{c}\text { Area } \\
\%\end{array}$ \\
\hline 1 & 15.060 & 326.187 & 433.257 & 97.09 \\
2 & 19.737 & 9.766 & 11.741 & 2.91 \\
\hline \multicolumn{2}{l|}{ Total: } & $\mathbf{3 3 5 . 9 5 3}$ & $\mathbf{4 4 4 . 9 9 8}$ & $\mathbf{1 0 0 . 0 0}$ \\
\hline
\end{tabular}




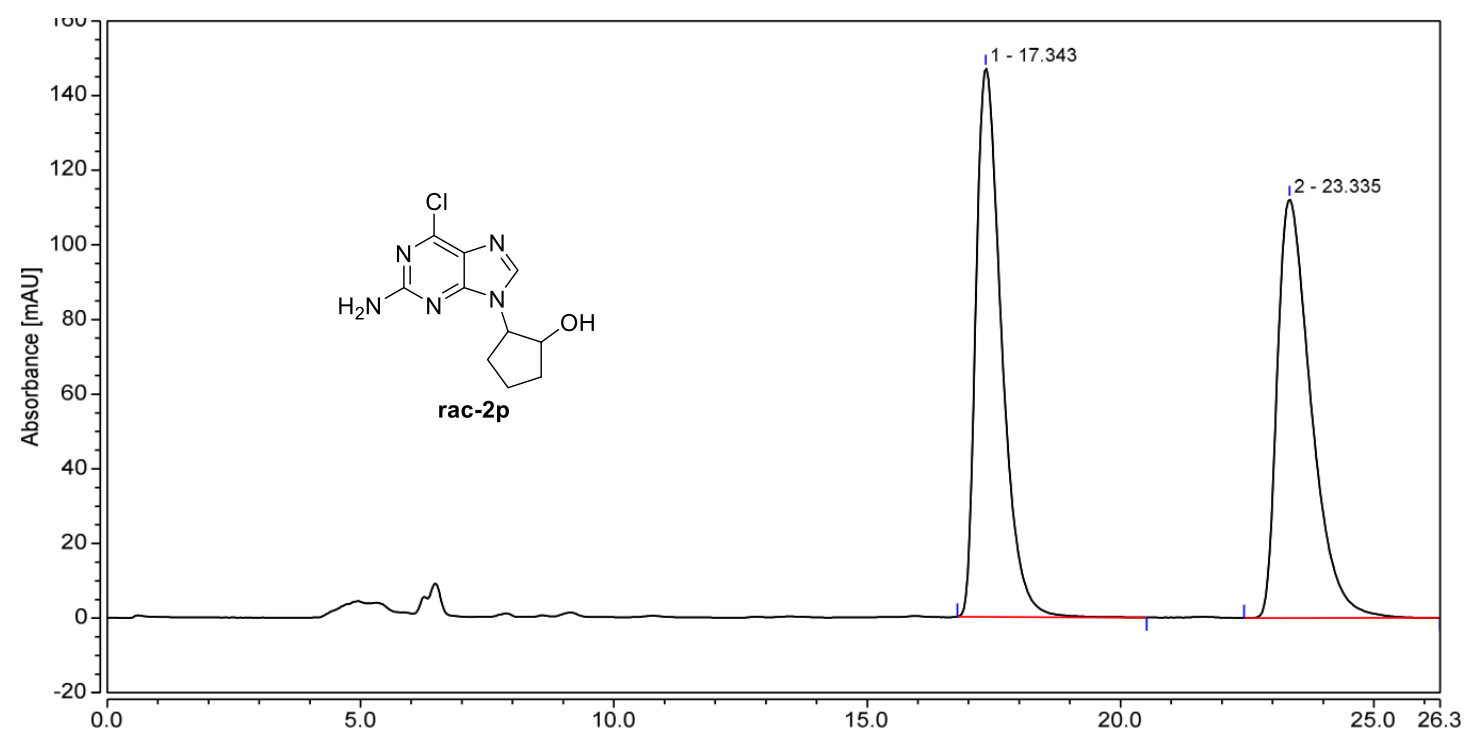

\begin{tabular}{|l|c|c|c|c|}
\hline Peak & $\begin{array}{c}\text { Retention Time } \\
\mathrm{min}\end{array}$ & $\begin{array}{c}\text { Area } \\
\mathrm{mAU} \text { min }\end{array}$ & $\begin{array}{c}\text { Height } \\
\mathrm{mAU}\end{array}$ & $\begin{array}{c}\text { Area } \\
\%\end{array}$ \\
\hline 1 & 17.343 & 86.048 & 147.073 & 49.84 \\
2 & 23.335 & 86.616 & 112.203 & 50.16 \\
\hline \multicolumn{2}{l|}{ Total: } & $\mathbf{1 7 2 . 6 6 4}$ & $\mathbf{2 5 9 . 2 7 6}$ & $\mathbf{1 0 0 . 0 0}$ \\
\hline
\end{tabular}

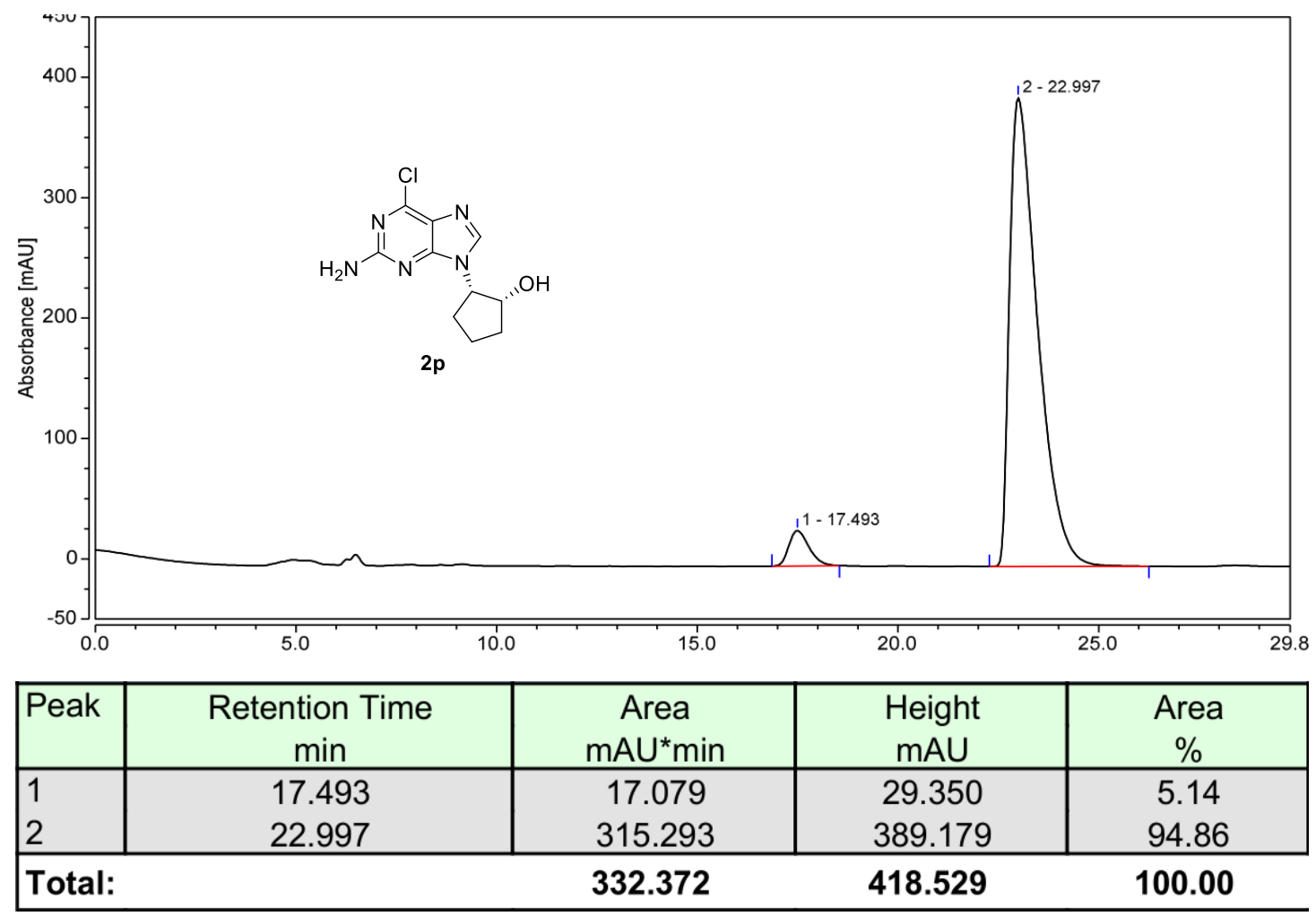




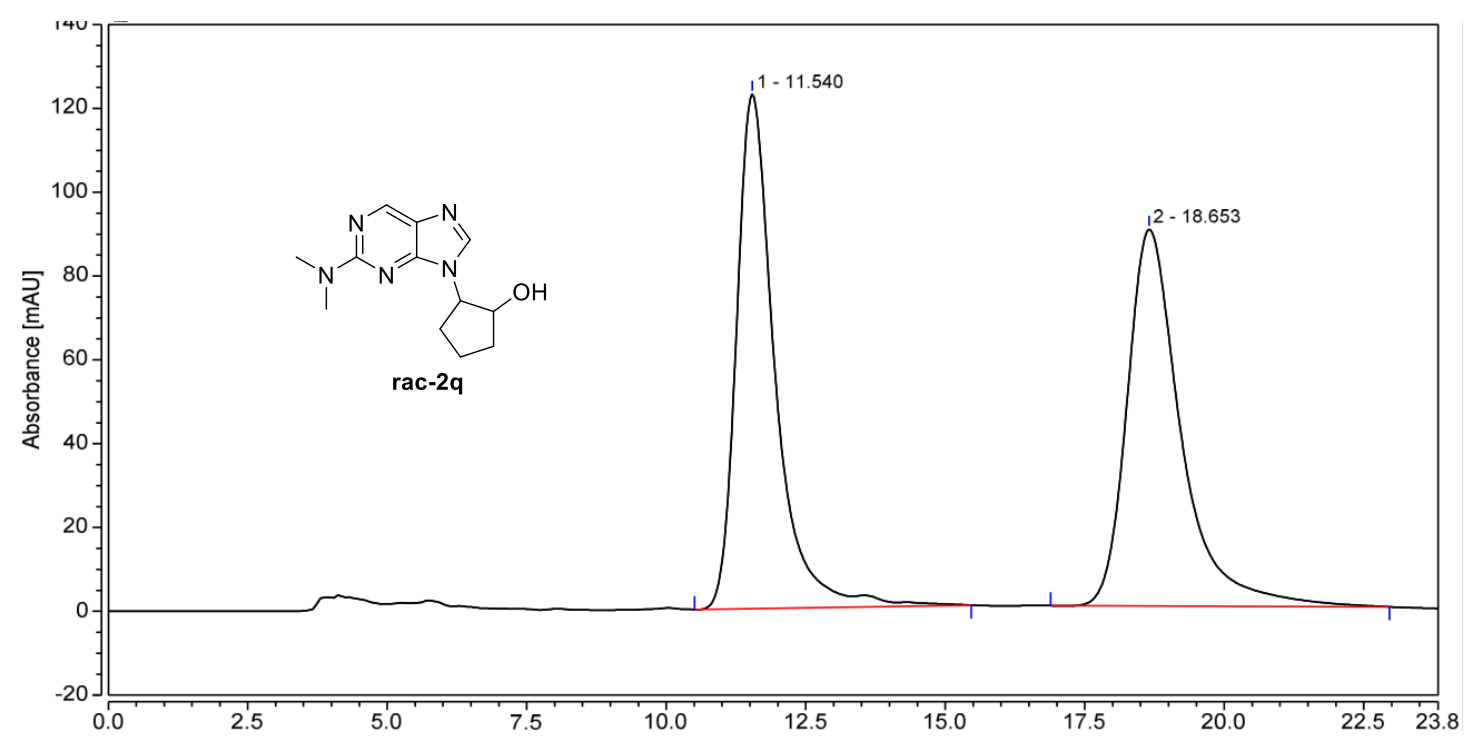

\begin{tabular}{|l|c|c|c|c|}
\hline Peak & $\begin{array}{c}\text { Retention Time } \\
\min \end{array}$ & $\begin{array}{c}\text { Area } \\
\mathrm{mAU}^{*} \min \end{array}$ & $\begin{array}{c}\text { Height } \\
\mathrm{mAU}\end{array}$ & $\begin{array}{c}\text { Area } \\
\%\end{array}$ \\
\hline 1 & 11.540 & 98.652 & 122.739 & 49.47 \\
2 & 18.653 & 100.781 & 89.891 & 50.53 \\
\hline \multicolumn{2}{l|}{ Total: } & $\mathbf{1 9 9 . 4 3 3}$ & $\mathbf{2 1 2 . 6 3 0}$ & $\mathbf{1 0 0 . 0 0}$ \\
\hline
\end{tabular}

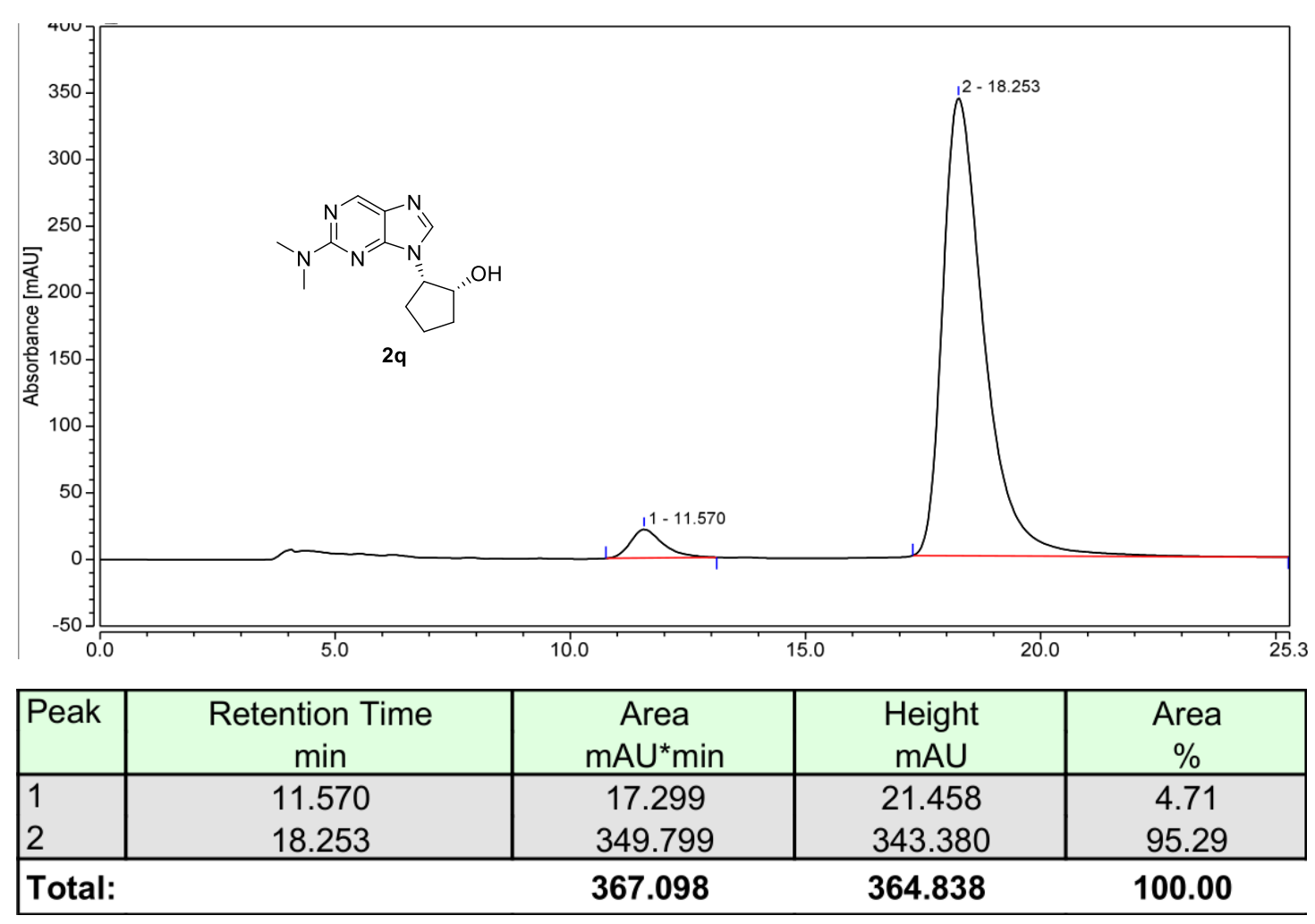




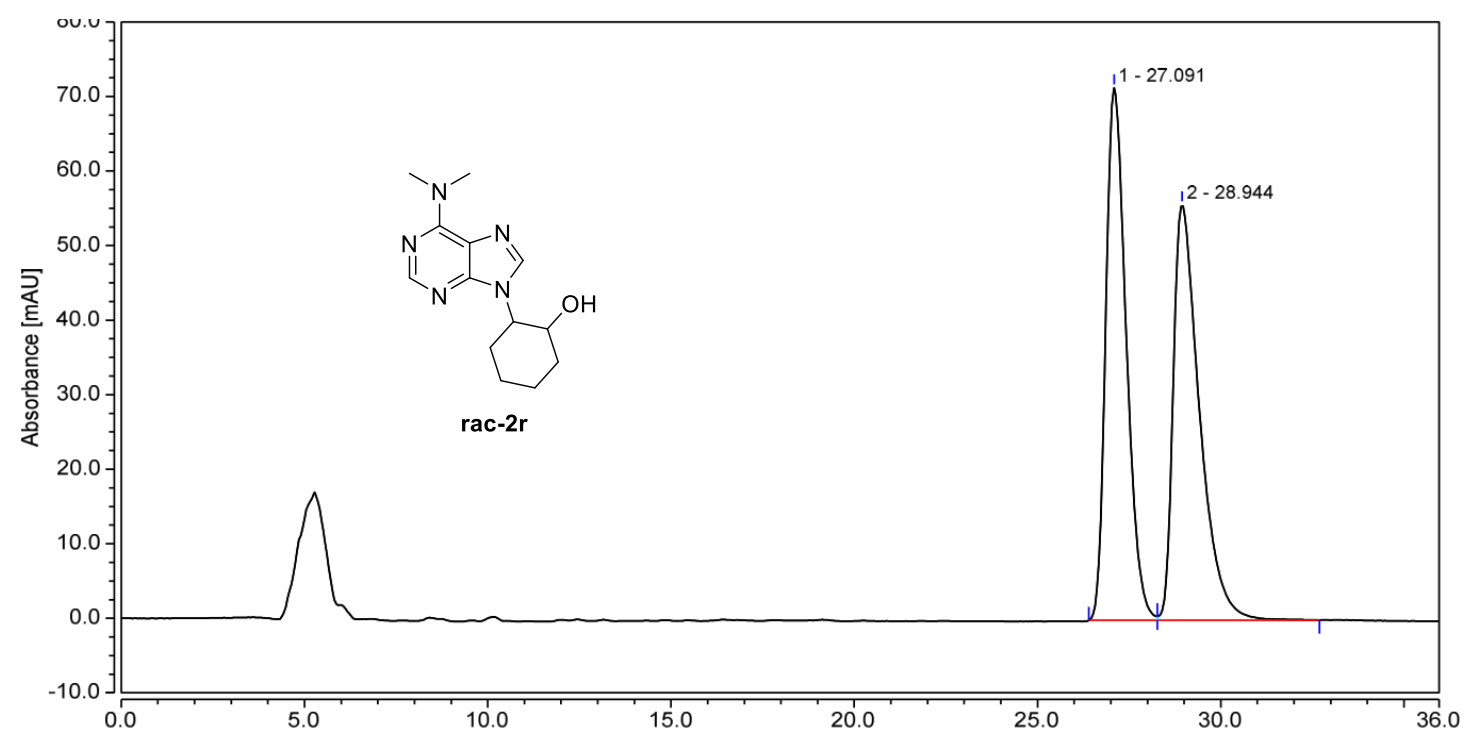

\begin{tabular}{|l|c|c|c|c|}
\hline Peak & $\begin{array}{c}\text { Retention Time } \\
\text { min }\end{array}$ & $\begin{array}{c}\text { Area } \\
\mathrm{mAU}^{*} \min \end{array}$ & $\begin{array}{c}\text { Height } \\
\mathrm{mAU}\end{array}$ & $\begin{array}{c}\text { Area } \\
\%\end{array}$ \\
\hline 1 & 27.091 & 46.102 & 71.425 & 49.88 \\
2 & 28.944 & 46.315 & 55.763 & 50.12 \\
\hline \multicolumn{2}{l|}{ Total: } & $\mathbf{9 2 . 4 1 7}$ & $\mathbf{1 2 7 . 1 8 8}$ & $\mathbf{1 0 0 . 0 0}$ \\
\hline
\end{tabular}

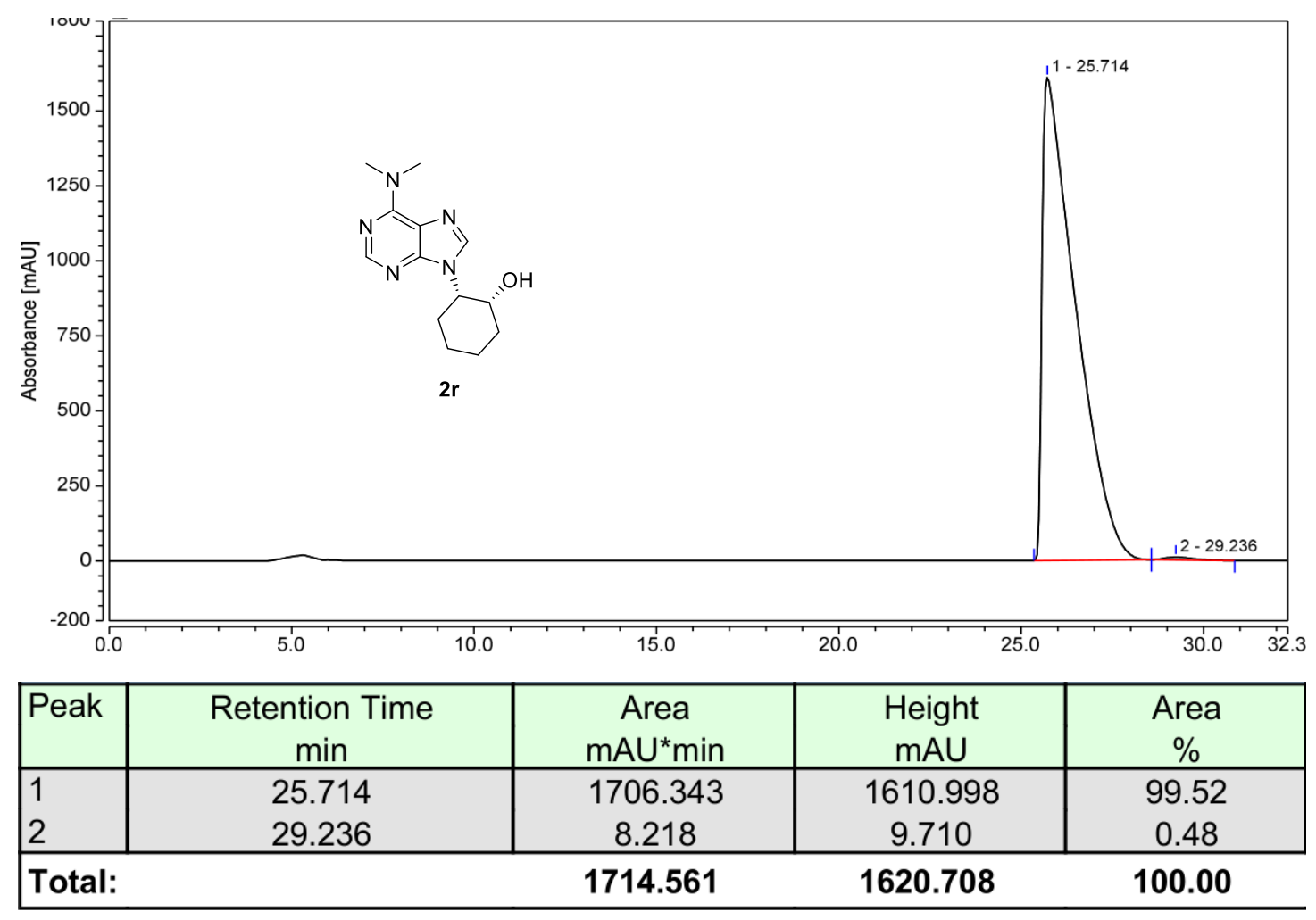




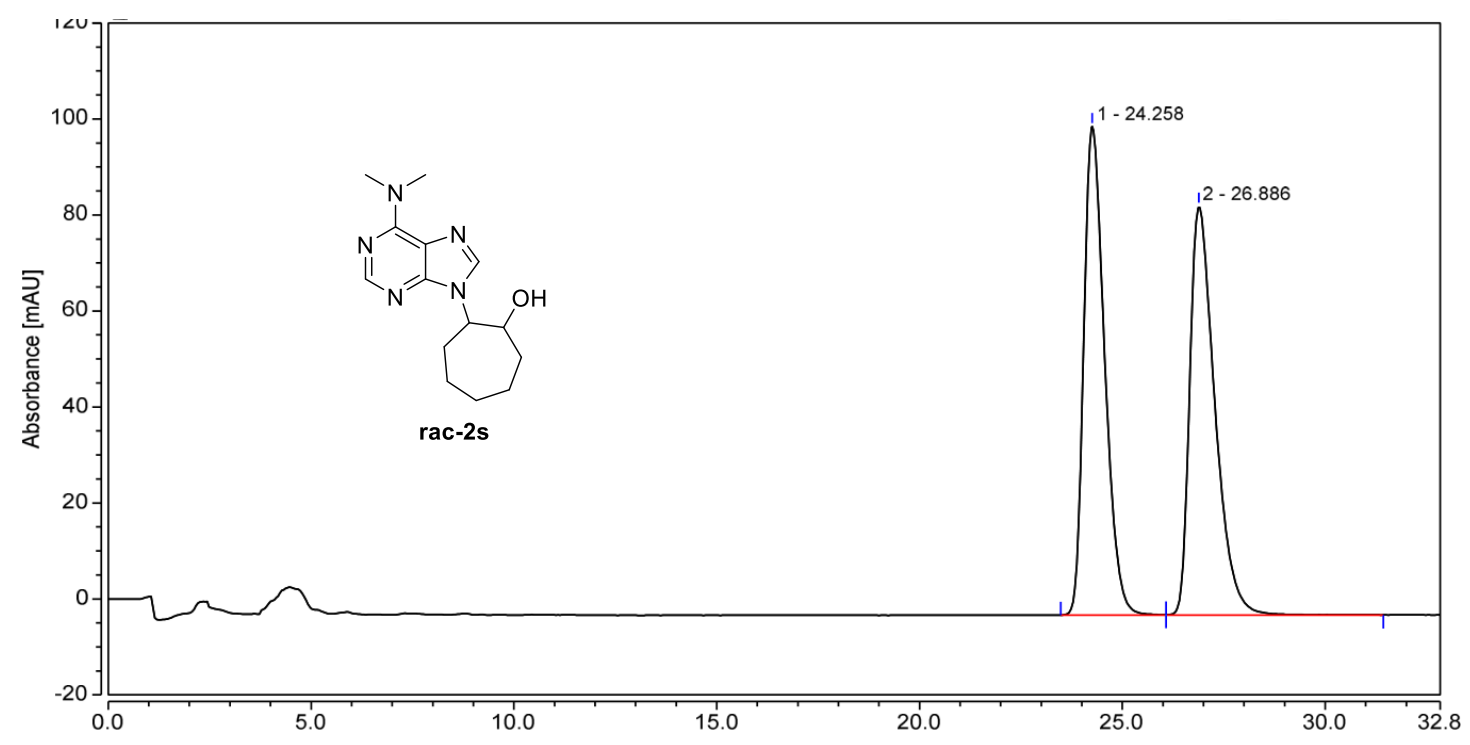

\begin{tabular}{|l|c|c|c|c|}
\hline Peak & $\begin{array}{c}\text { Retention Time } \\
\min \end{array}$ & $\begin{array}{c}\text { Area } \\
\mathrm{mAU}^{*} \min \end{array}$ & $\begin{array}{c}\text { Height } \\
\mathrm{mAU}\end{array}$ & $\begin{array}{c}\text { Area } \\
\%\end{array}$ \\
\hline 1 & 24.258 & 60.664 & 101.831 & 49.80 \\
2 & 26.886 & 61.139 & 85.258 & 50.20 \\
\hline \multicolumn{2}{l|}{ Total: } & $\mathbf{1 2 1 . 8 0 3}$ & $\mathbf{1 8 7 . 0 8 9}$ & $\mathbf{1 0 0 . 0 0}$ \\
\hline
\end{tabular}

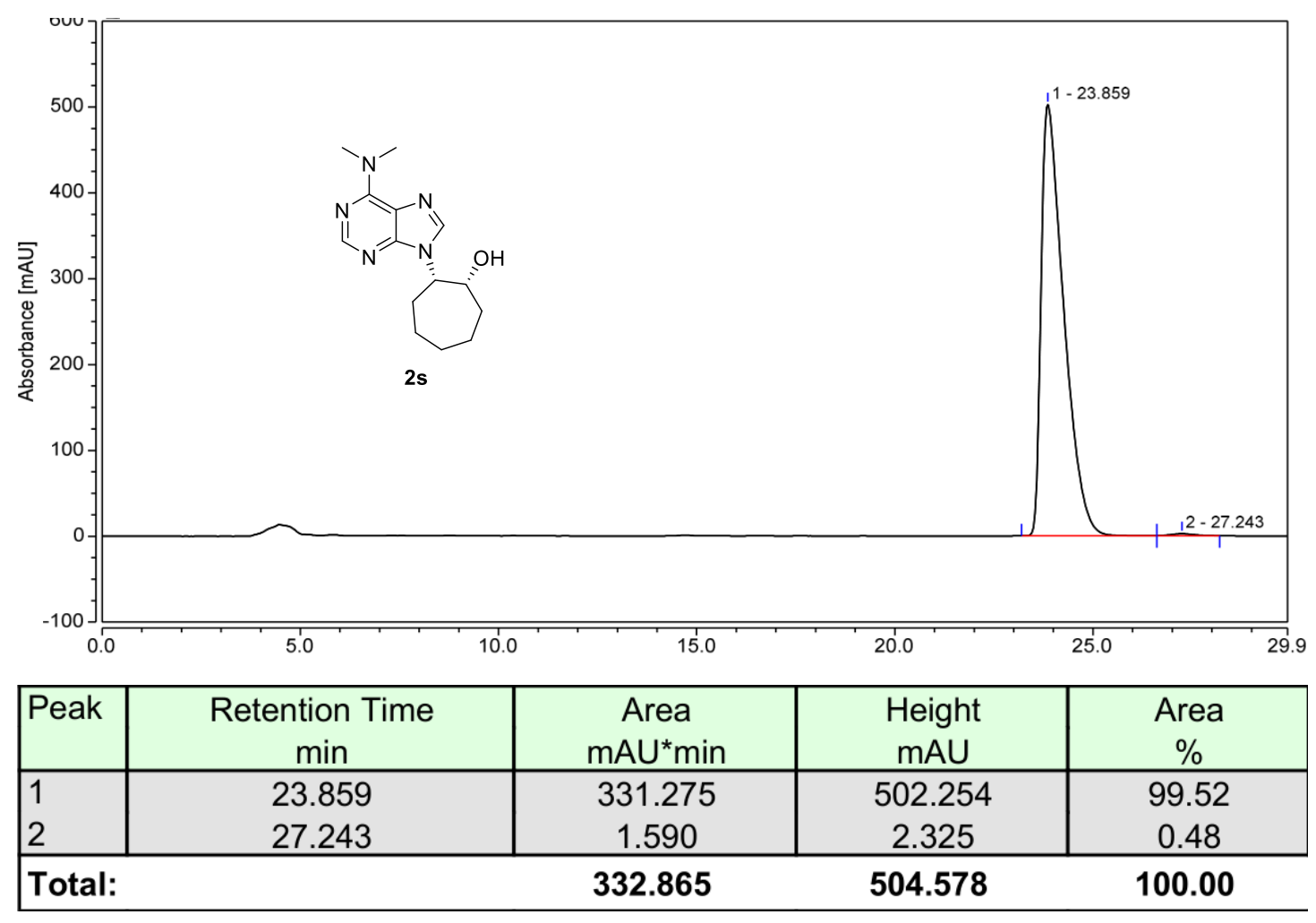




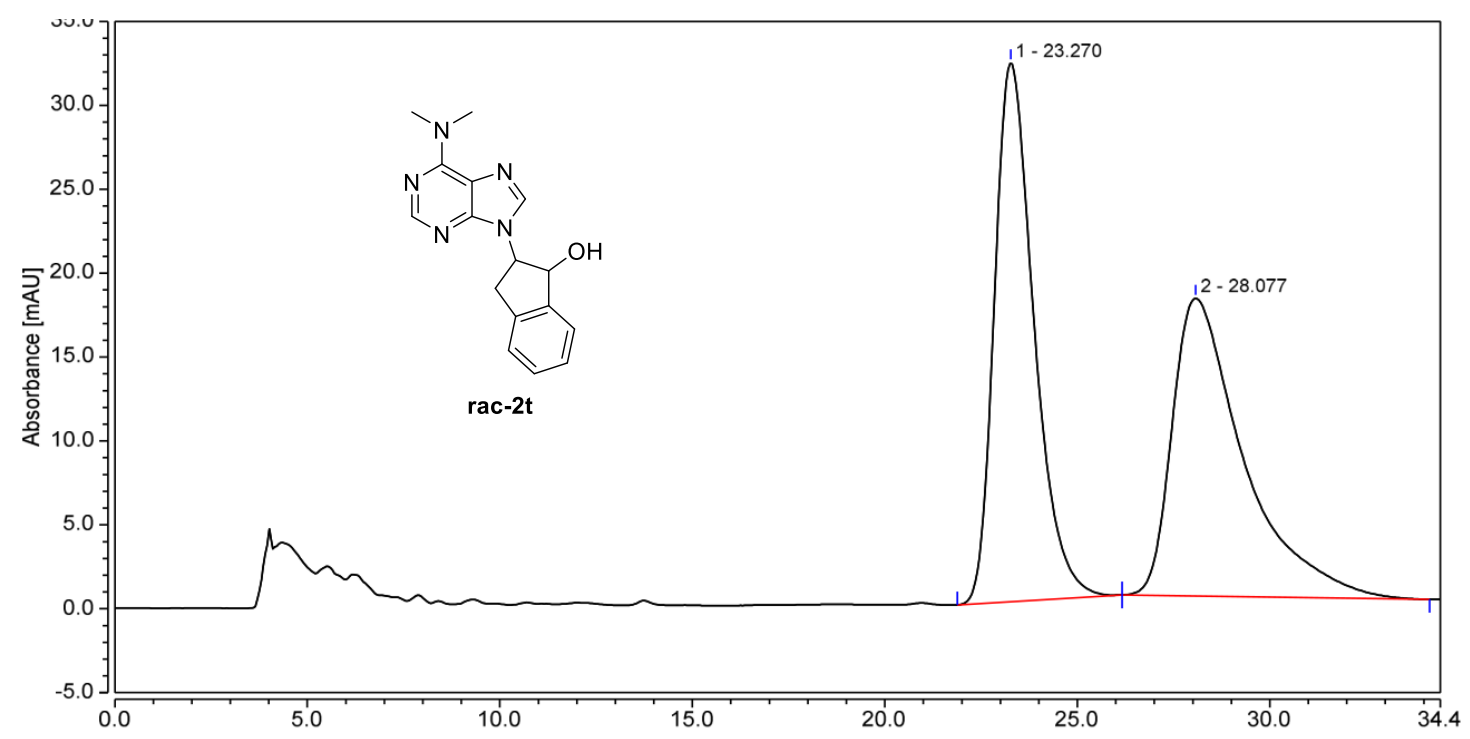

\begin{tabular}{|l|c|c|c|c|}
\hline Peak & $\begin{array}{c}\text { Retention Time } \\
\mathrm{min}\end{array}$ & $\begin{array}{c}\text { Area } \\
\mathrm{mAU} \text { min }\end{array}$ & $\begin{array}{c}\text { Height } \\
\mathrm{mAU}\end{array}$ & $\begin{array}{c}\text { Area } \\
\%\end{array}$ \\
\hline 1 & 23.270 & 38.201 & 32.120 & 50.08 \\
2 & 28.077 & 38.081 & 17.753 & 49.92 \\
\hline \multicolumn{2}{l|}{ Total: } & $\mathbf{7 6 . 2 8 3}$ & $\mathbf{4 9 . 8 7 3}$ & $\mathbf{1 0 0 . 0 0}$ \\
\hline
\end{tabular}

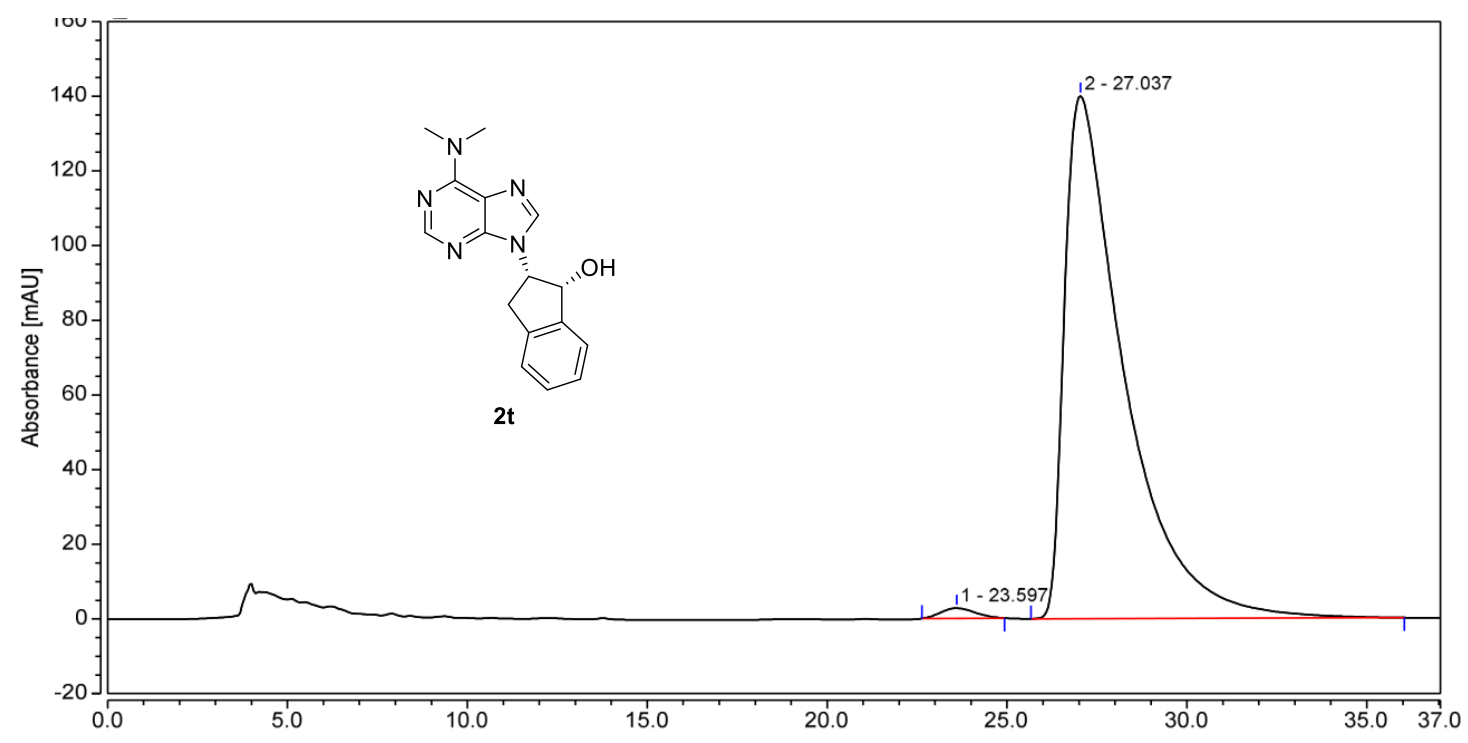

\begin{tabular}{|l|c|c|c|c|}
\hline Peak & $\begin{array}{c}\text { Retention Time } \\
\text { min }\end{array}$ & $\begin{array}{c}\text { Area } \\
\mathrm{mAU}^{*} \min \end{array}$ & $\begin{array}{c}\text { Height } \\
\mathrm{mAU}\end{array}$ & $\begin{array}{c}\text { Area } \\
\%\end{array}$ \\
\hline 1 & 23.597 & 3.034 & 2.728 & 1.11 \\
2 & 27.037 & 270.186 & 139.986 & 98.89 \\
\hline \multicolumn{2}{l|}{ Total: } & $\mathbf{2 7 3 . 2 2 0}$ & $\mathbf{1 4 2 . 7 1 4}$ & $\mathbf{1 0 0 . 0 0}$ \\
\hline
\end{tabular}




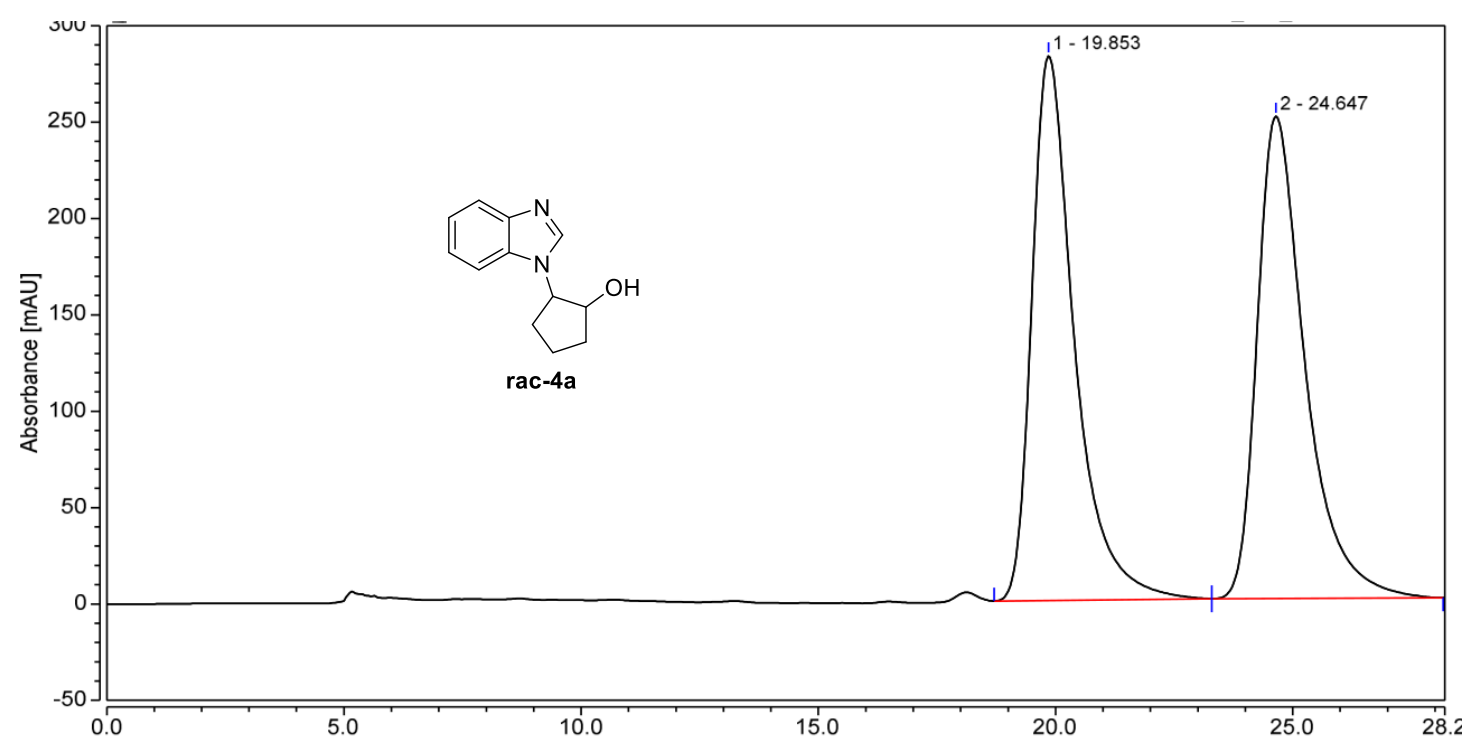

\begin{tabular}{|l|c|c|c|c|c|}
\hline Peak & $\begin{array}{c}\text { Retention Time } \\
\text { min }\end{array}$ & $\begin{array}{c}\text { Area } \\
\mathrm{mAU}^{*} \min \end{array}$ & $\begin{array}{c}\text { Height } \\
\mathrm{mAU}\end{array}$ & $\begin{array}{c}\text { Area } \\
\%\end{array}$ & $\begin{array}{c}\text { Height } \\
\%\end{array}$ \\
\hline 1 & 19.853 & 294.595 & 282.615 & 50.13 & 53.03 \\
2 & 24.647 & 293.113 & 250.271 & 49.87 & 46.97 \\
\hline \multicolumn{2}{l|}{ Total: } & $\mathbf{5 8 7 . 7 0 9}$ & $\mathbf{5 3 2 . 8 8 5}$ & $\mathbf{1 0 0 . 0 0}$ & $\mathbf{1 0 0 . 0 0}$ \\
\hline
\end{tabular}

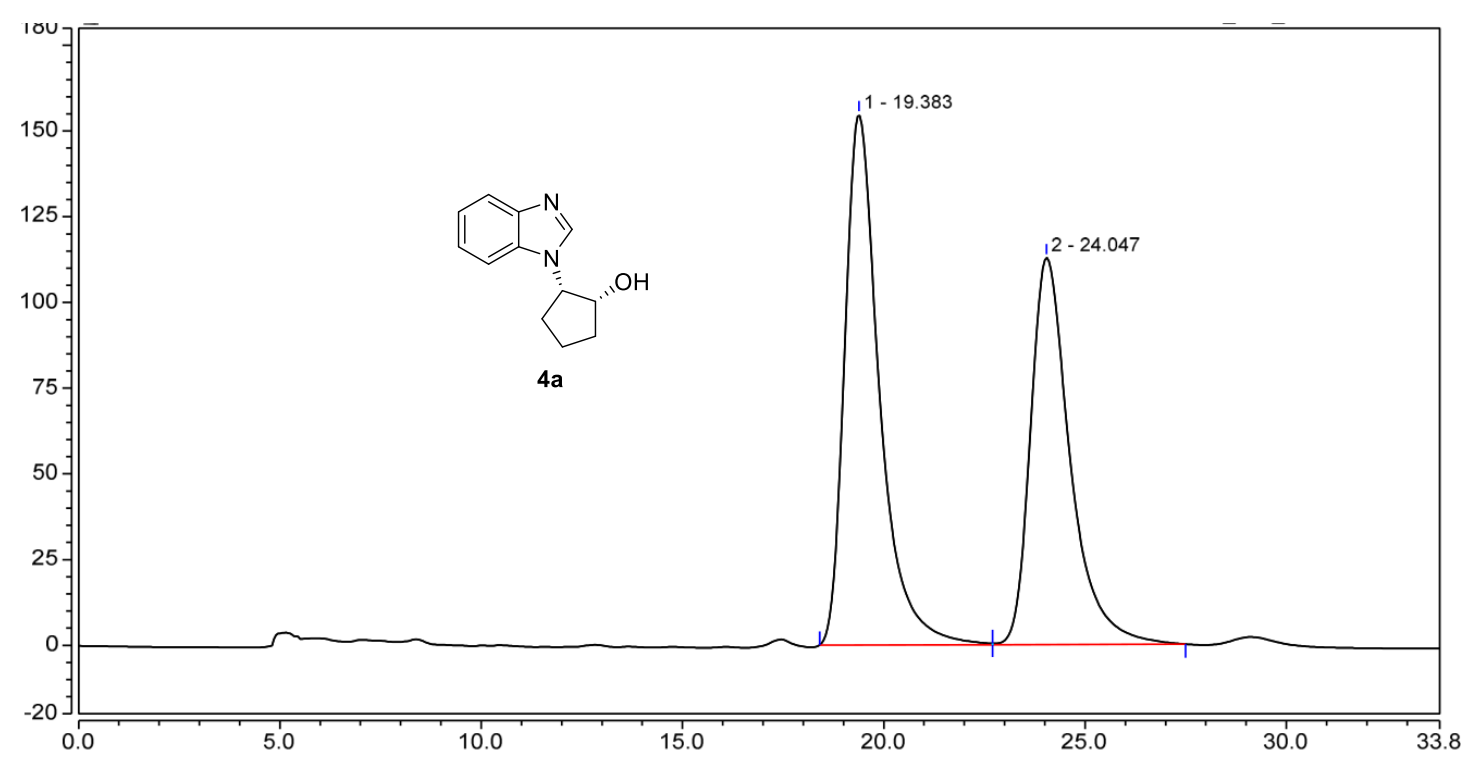

\begin{tabular}{|l|c|c|c|c|c|}
\hline Peak & $\begin{array}{c}\text { Retention Time } \\
\min \end{array}$ & $\begin{array}{c}\text { Area } \\
\mathrm{mAU}\end{array}$ & $\begin{array}{c}\text { Height } \\
\mathrm{mAU}\end{array}$ & $\begin{array}{c}\text { Area } \\
\%\end{array}$ & $\begin{array}{c}\text { Height } \\
\%\end{array}$ \\
\hline 1 & 19.383 & 155.119 & 154.694 & 54.67 & 57.83 \\
2 & 24.047 & 128.632 & 112.804 & 45.33 & 42.17 \\
\hline \multicolumn{2}{|l}{ Total: } & $\mathbf{2 8 3 . 7 5 1}$ & $\mathbf{2 6 7 . 4 9 8}$ & $\mathbf{1 0 0 . 0 0}$ & $\mathbf{1 0 0 . 0 0}$ \\
\hline
\end{tabular}




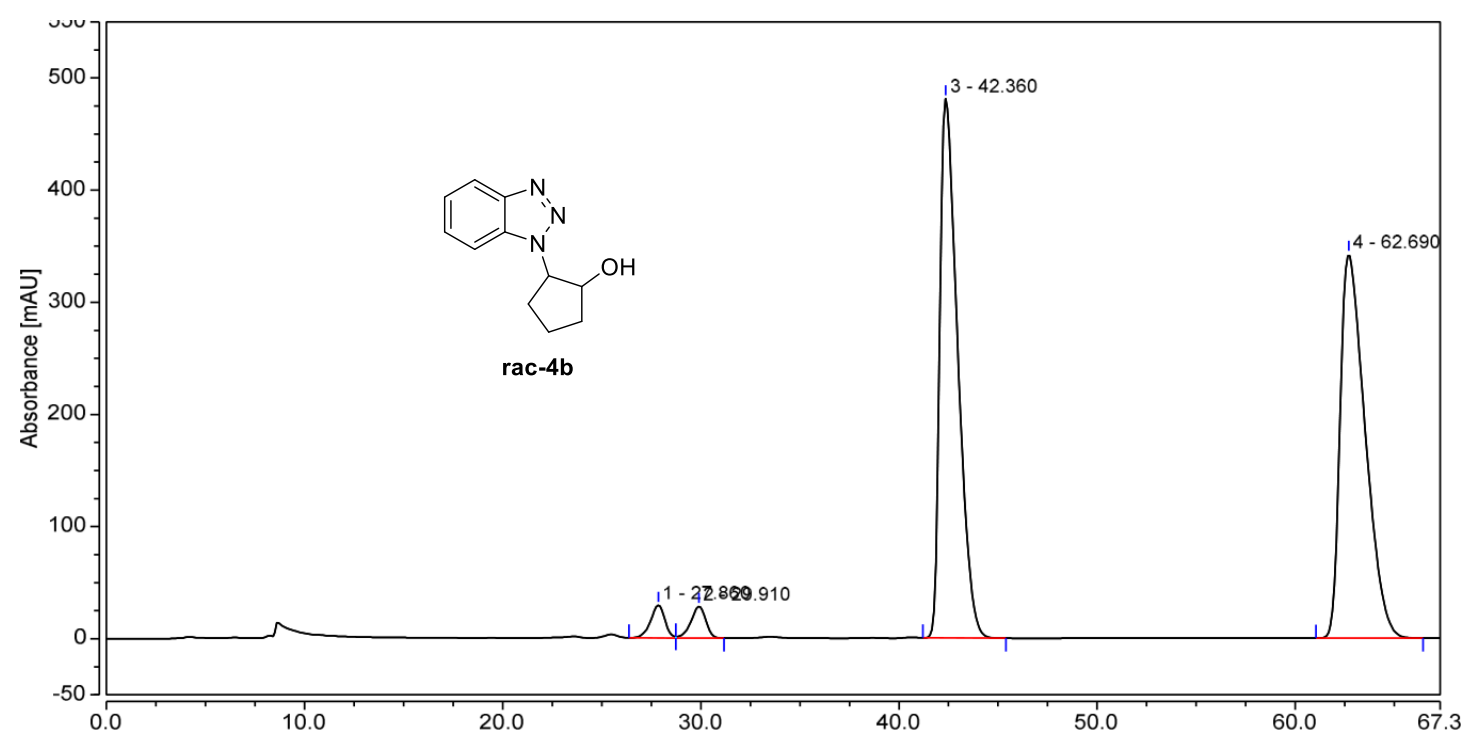

\begin{tabular}{|l|c|c|c|c|}
\hline Peak & $\begin{array}{c}\text { Retention Time } \\
\min \end{array}$ & $\begin{array}{c}\text { Area } \\
\mathrm{mAU}^{*} \min \end{array}$ & $\begin{array}{c}\text { Height } \\
\mathrm{mAU}\end{array}$ & $\begin{array}{c}\text { Area } \\
\%\end{array}$ \\
\hline 1 & 27.860 & 24.550 & 29.290 & 2.33 \\
2 & 29.910 & 24.554 & 28.254 & 2.33 \\
3 & 42.360 & 501.918 & 480.685 & 47.73 \\
4 & 62.690 & 500.554 & 342.296 & 47.60 \\
\hline Total: & $\mathbf{1 0 5 1 . 5 7 6}$ & $\mathbf{8 8 0 . 5 2 4}$ & $\mathbf{1 0 0 . 0 0}$ \\
\hline
\end{tabular}

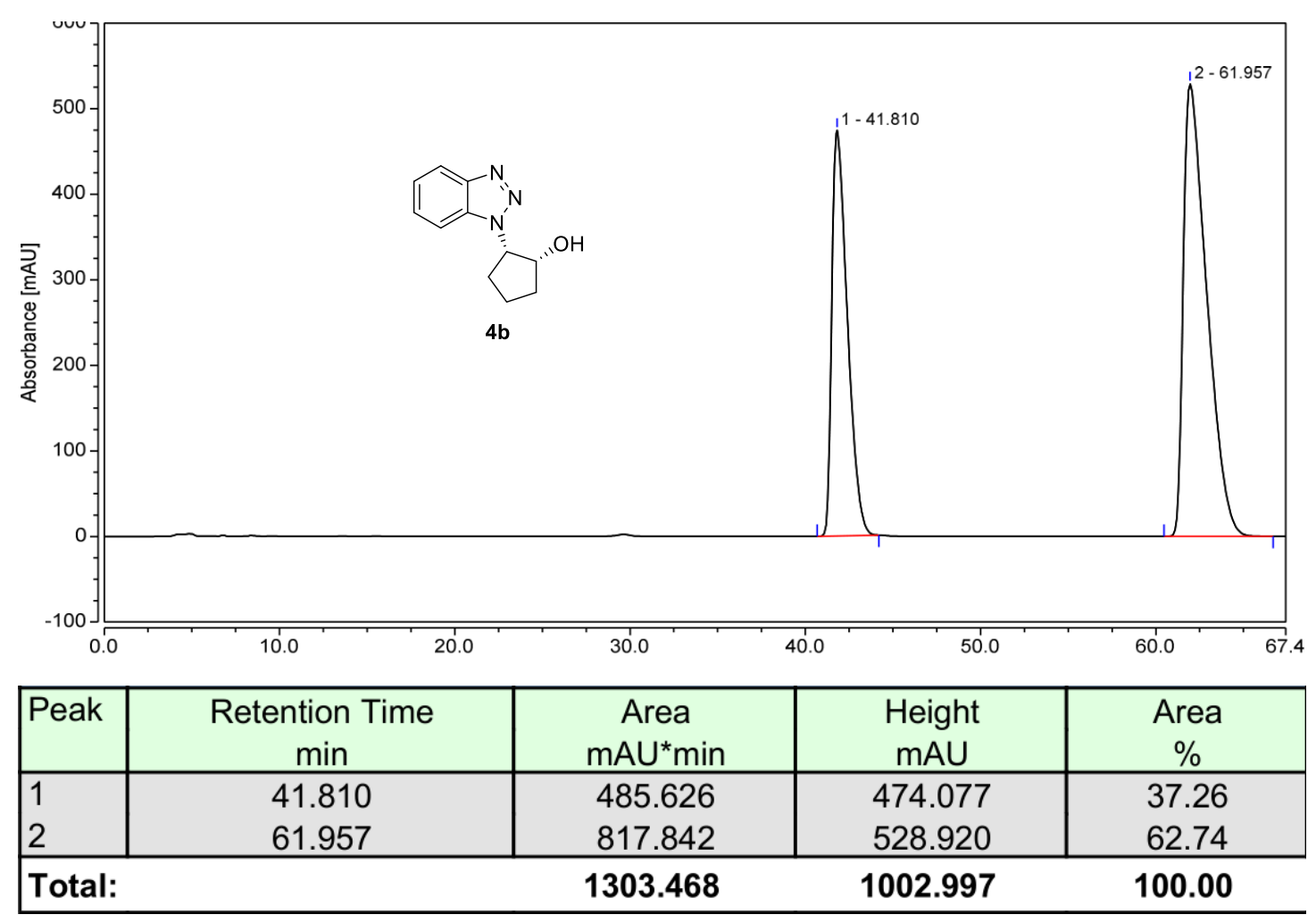




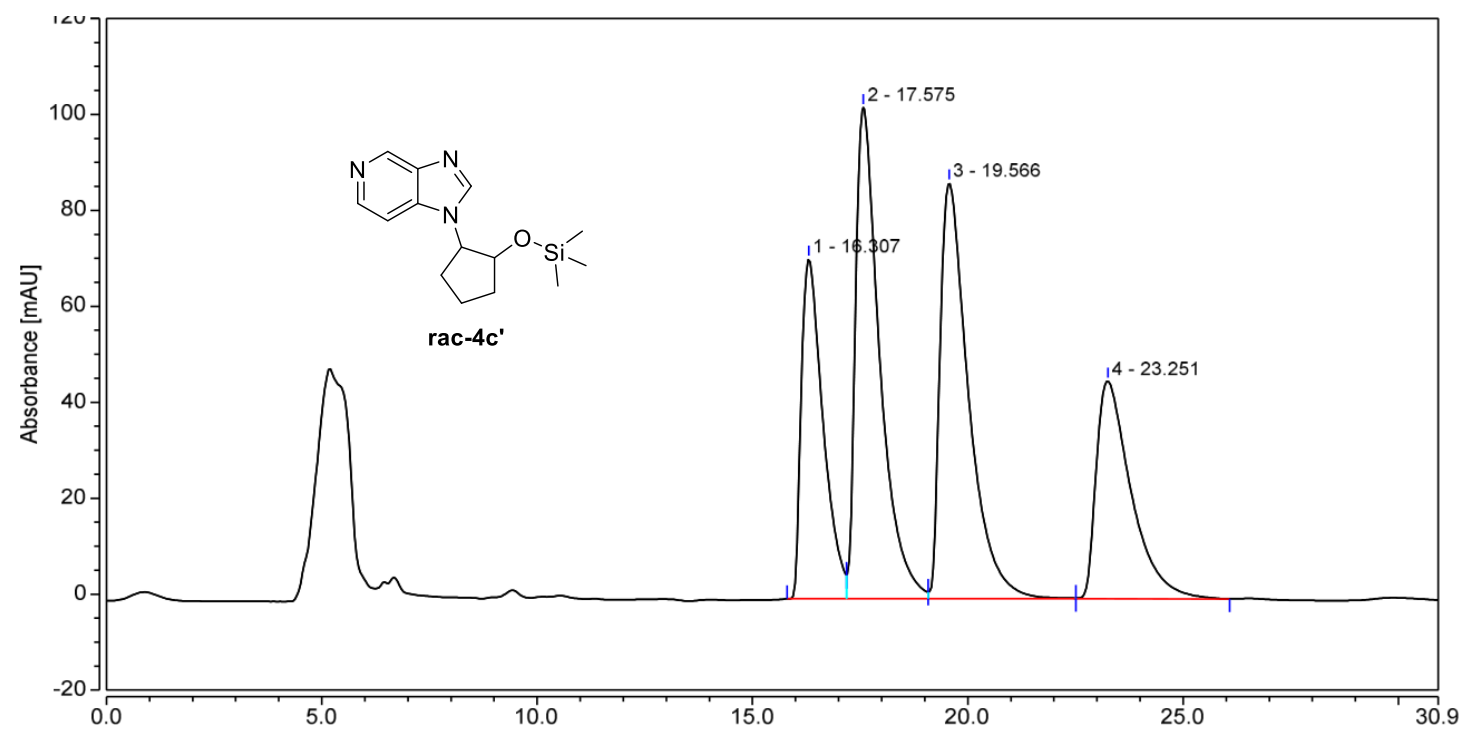

\begin{tabular}{|l|c|c|c|c|}
\hline Peak & $\begin{array}{c}\text { Retention Time } \\
\text { min }\end{array}$ & $\begin{array}{c}\text { Area } \\
\mathrm{mAU}^{*} \min \end{array}$ & $\begin{array}{c}\text { Height } \\
\mathrm{mAU}\end{array}$ & $\begin{array}{c}\text { Area } \\
\%\end{array}$ \\
\hline 1 & 16.307 & 41.527 & 70.854 & 19.08 \\
2 & 17.575 & 66.994 & 102.444 & 30.78 \\
3 & 19.566 & 66.564 & 86.766 & 30.58 \\
4 & 23.251 & 42.589 & 45.412 & 19.57 \\
\hline Total & $\mathbf{2 1 7 . 6 7 3}$ & $\mathbf{3 0 5 . 4 7 6}$ & $\mathbf{1 0 0 . 0 0}$ \\
\hline
\end{tabular}

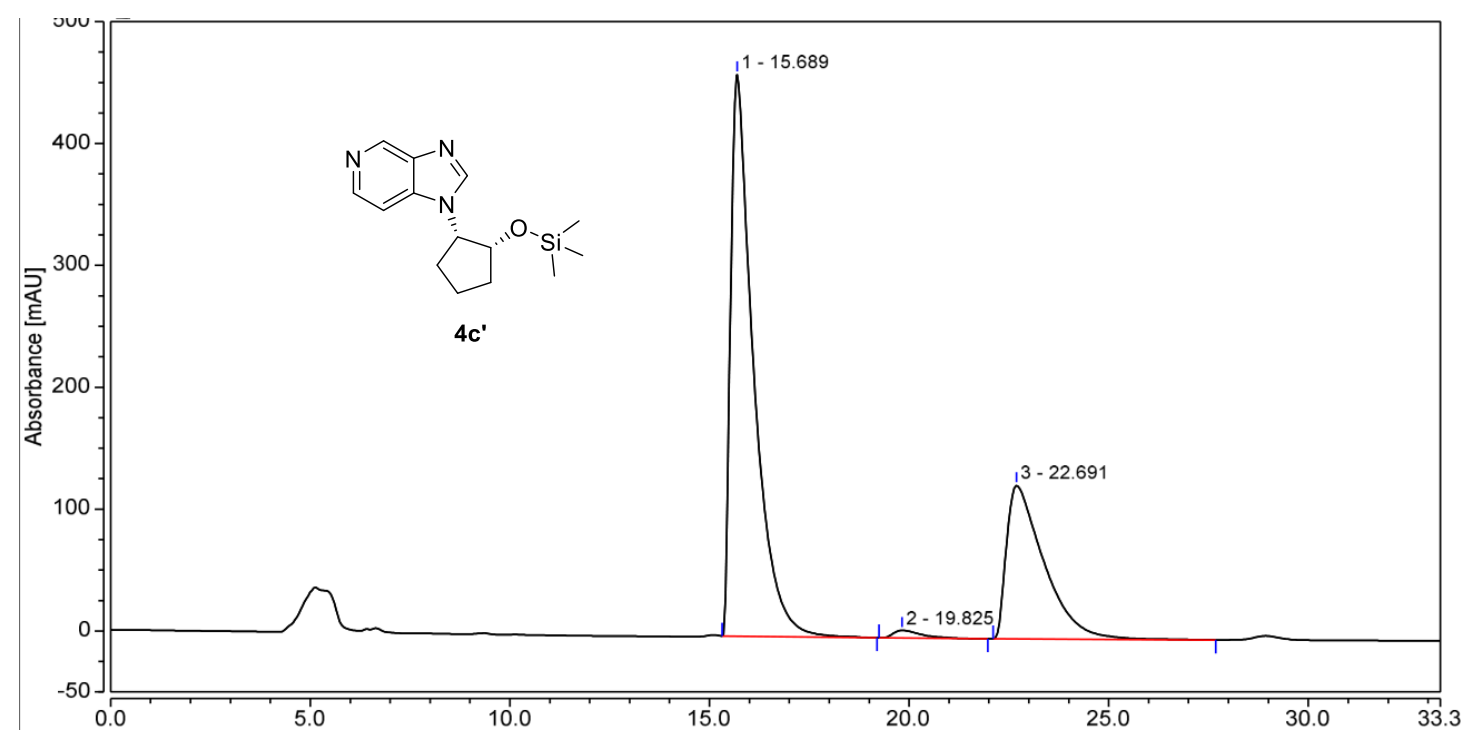

\begin{tabular}{|l|c|c|c|c|}
\hline Peak & $\begin{array}{c}\text { Retention Time } \\
\text { min }\end{array}$ & $\begin{array}{c}\text { Area } \\
\mathrm{mAU}^{*} \min \end{array}$ & $\begin{array}{c}\text { Height } \\
\mathrm{mAU}\end{array}$ & $\begin{array}{c}\text { Area } \\
\%\end{array}$ \\
\hline 1 & 15.689 & 306.603 & 460.553 & 68.30 \\
2 & 19.825 & 5.215 & 6.377 & 1.16 \\
3 & 22.691 & 137.075 & 125.812 & 30.54 \\
\hline Total & $\mathbf{4 4 8 . 8 9 3}$ & $\mathbf{5 9 2 . 7 4 2}$ & $\mathbf{1 0 0 . 0 0}$ \\
\hline
\end{tabular}




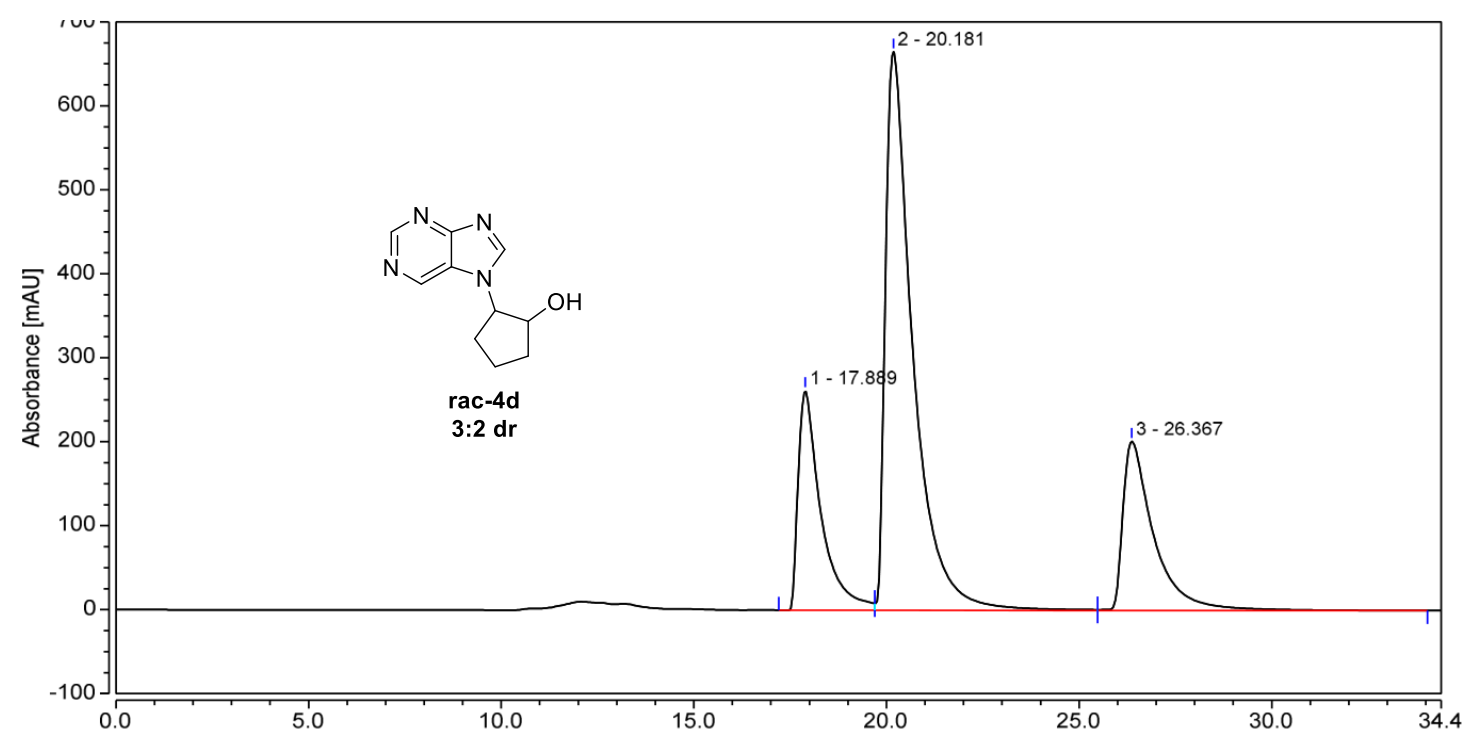

\begin{tabular}{|l|c|c|c|c|}
\hline Peak & $\begin{array}{c}\text { Retention Time } \\
\min \end{array}$ & $\begin{array}{c}\text { Area } \\
\mathrm{mAU}^{*} \min \end{array}$ & $\begin{array}{c}\text { Height } \\
\mathrm{mAU}\end{array}$ & $\begin{array}{c}\text { Area } \\
\%\end{array}$ \\
\hline 1 & 17.889 & 178.431 & 261.743 & 19.43 \\
2 & 20.181 & 551.972 & 664.971 & 60.11 \\
3 & 26.367 & 187.866 & 201.552 & 20.46 \\
\hline \multicolumn{2}{l|}{ Total: } & $\mathbf{9 1 8 . 2 6 9}$ & $\mathbf{1 1 2 8 . 2 6 6}$ & $\mathbf{1 0 0 . 0 0}$ \\
\hline
\end{tabular}

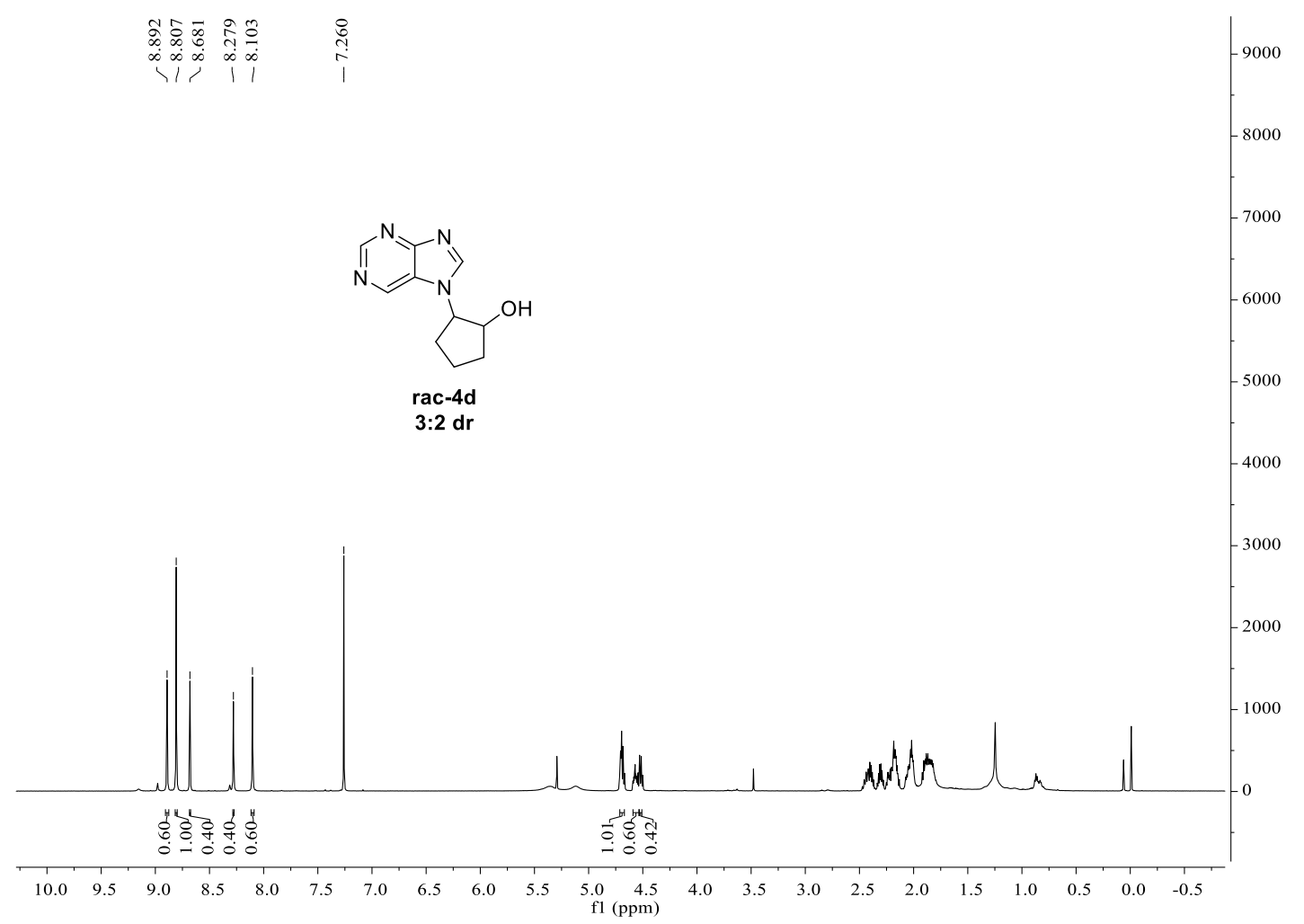




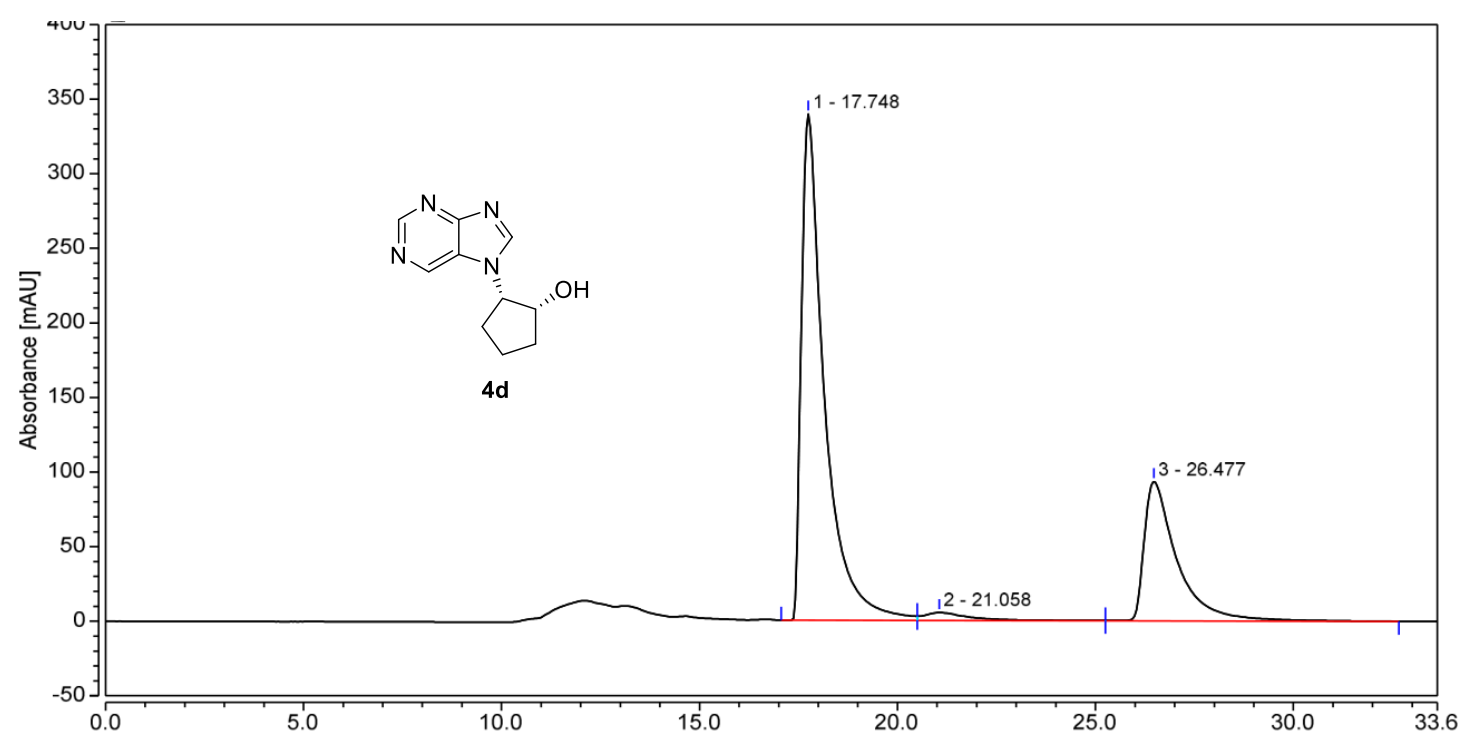

\begin{tabular}{|l|c|c|c|c|}
\hline Peak & $\begin{array}{c}\text { Retention Time } \\
\min \end{array}$ & $\begin{array}{c}\text { Area } \\
\mathrm{mAU}^{*} \min \end{array}$ & $\begin{array}{c}\text { Height } \\
\mathrm{mAU}\end{array}$ & $\begin{array}{c}\text { Area } \\
\%\end{array}$ \\
\hline 1 & 17.748 & 229.808 & 339.436 & 70.36 \\
2 & 21.058 & 7.132 & 5.336 & 2.18 \\
3 & 26.477 & 89.688 & 93.380 & 27.46 \\
\hline \multicolumn{2}{l}{ Total: } & $\mathbf{3 2 6 . 6 2 9}$ & $\mathbf{4 3 8 . 1 5 2}$ & $\mathbf{1 0 0 . 0 0}$ \\
\hline
\end{tabular}




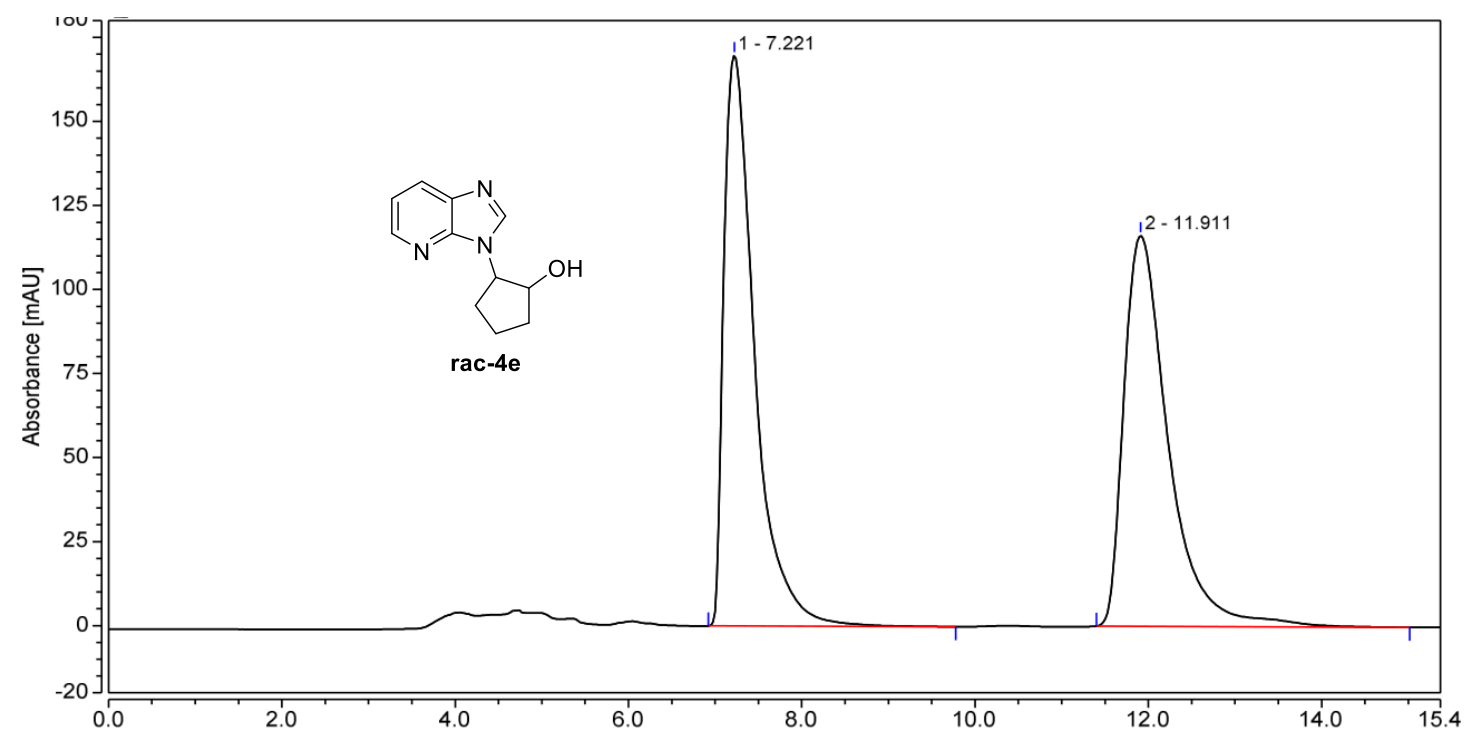

\begin{tabular}{|l|c|c|c|c|}
\hline Peak & $\begin{array}{c}\text { Retention Time } \\
\mathrm{min}\end{array}$ & $\begin{array}{c}\text { Area } \\
\mathrm{mAU} \text { min }\end{array}$ & $\begin{array}{c}\text { Height } \\
\mathrm{mAU}\end{array}$ & $\begin{array}{c}\text { Area } \\
\%\end{array}$ \\
\hline 1 & 7.221 & 70.063 & 169.763 & 50.30 \\
2 & 11.911 & 69.230 & 116.242 & 49.70 \\
\hline \multicolumn{2}{|l|}{ Total: } & $\mathbf{1 3 9 . 2 9 3}$ & $\mathbf{2 8 6 . 0 0 5}$ & $\mathbf{1 0 0 . 0 0}$ \\
\hline
\end{tabular}

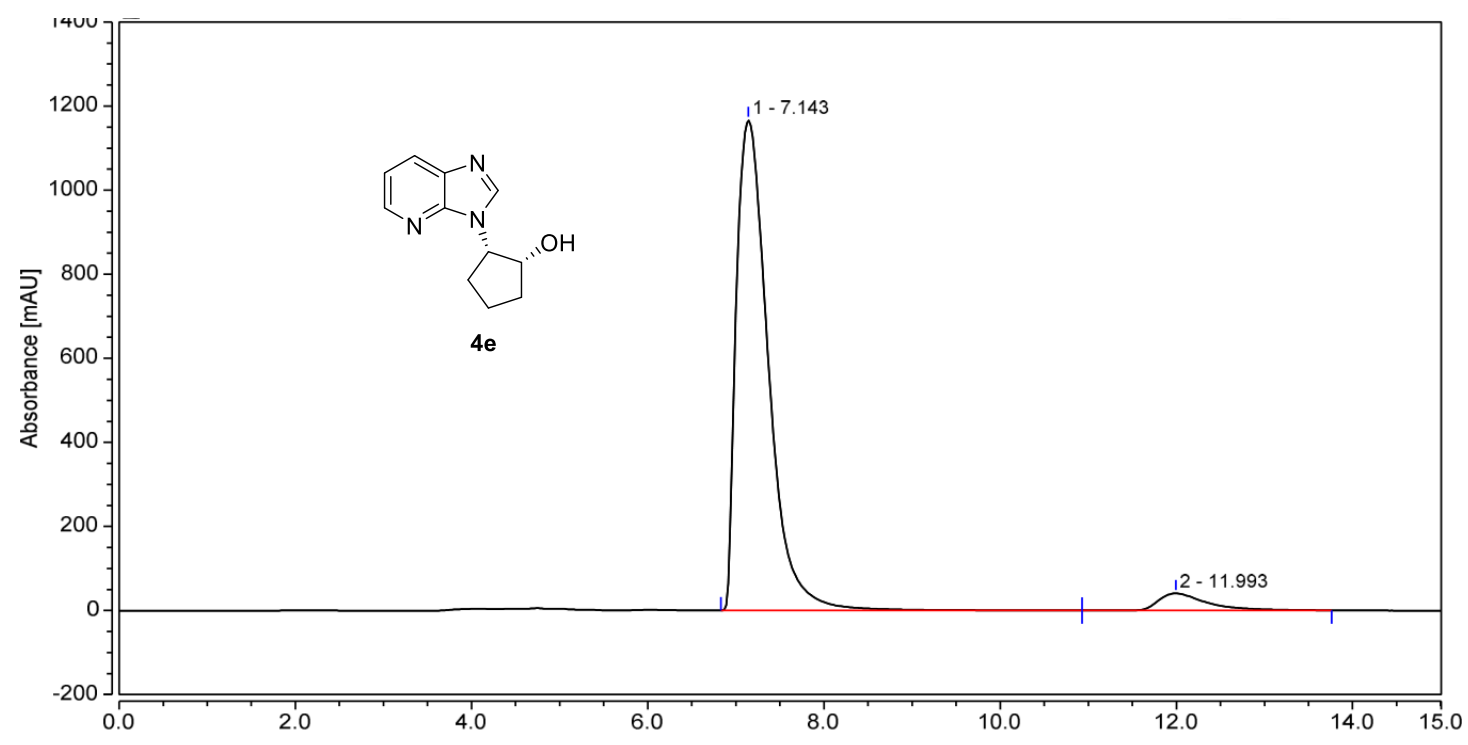

\begin{tabular}{|l|c|c|c|c|}
\hline Peak & $\begin{array}{c}\text { Retention Time } \\
\text { min }\end{array}$ & $\begin{array}{c}\text { Area } \\
\mathrm{mAU}^{*} \min \end{array}$ & $\begin{array}{c}\text { Height } \\
\mathrm{mAU}\end{array}$ & $\begin{array}{c}\text { Area } \\
\%\end{array}$ \\
\hline 1 & 7.143 & 492.182 & 1166.030 & 94.99 \\
2 & 11.993 & 25.940 & 40.725 & 5.01 \\
\hline \multicolumn{2}{l|}{} \\
\hline \multicolumn{2}{l|}{$\mathbf{5 1 8 . 1 2 2}$} & $\mathbf{1 2 0 6 . 7 5 4}$ & $\mathbf{1 0 0 . 0 0}$ \\
\hline
\end{tabular}




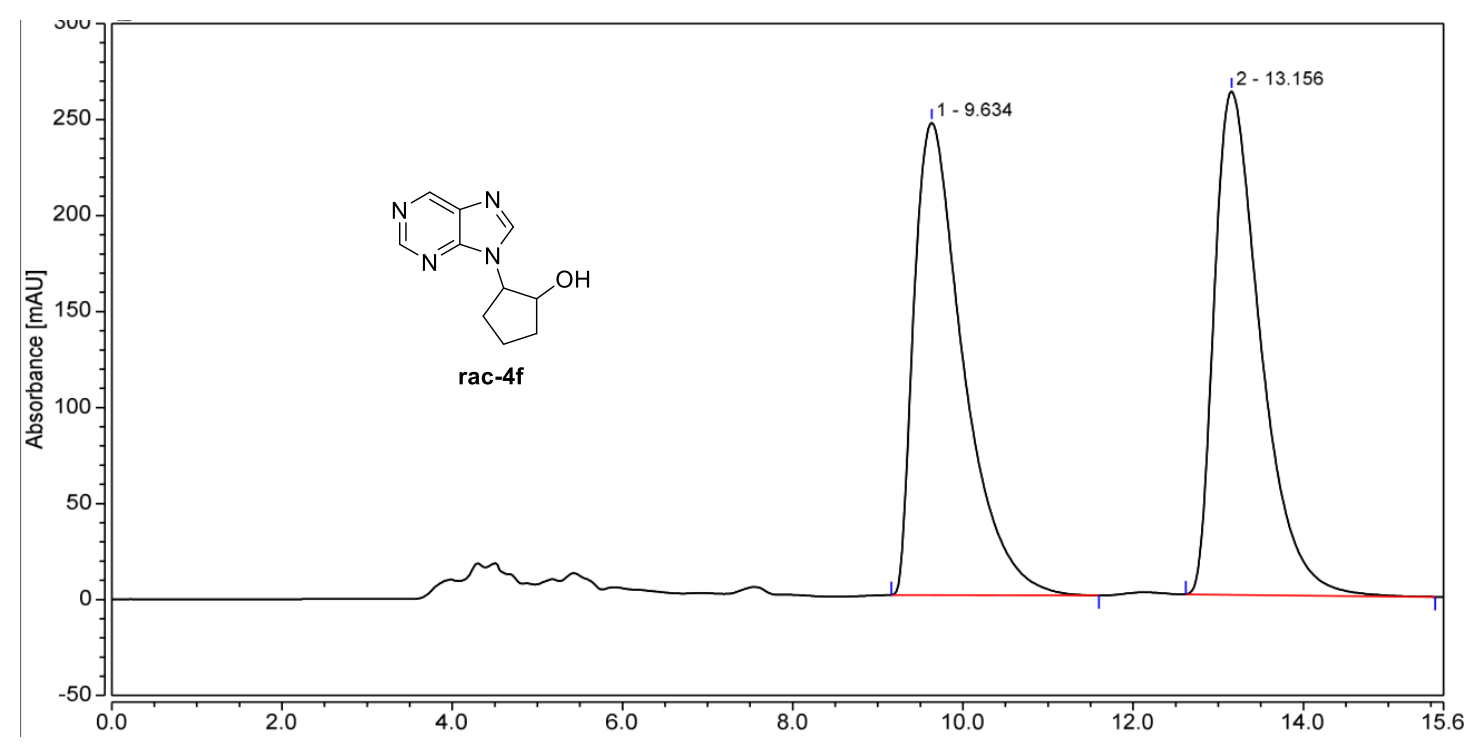

\begin{tabular}{|l|c|c|c|c|}
\hline Peak & $\begin{array}{c}\text { Retention Time } \\
\mathrm{min}\end{array}$ & $\begin{array}{c}\text { Area } \\
\mathrm{mAU}^{*} \min \end{array}$ & $\begin{array}{c}\text { Height } \\
\mathrm{mAU}\end{array}$ & $\begin{array}{c}\text { Area } \\
\%\end{array}$ \\
\hline 1 & 9.634 & 164.604 & 246.161 & 49.83 \\
2 & 13.156 & 165.759 & 262.354 & 50.17 \\
\hline \multicolumn{2}{l|}{ Total: } & $\mathbf{3 3 0 . 3 6 3}$ & $\mathbf{5 0 8 . 5 1 5}$ & $\mathbf{1 0 0 . 0 0}$ \\
\hline
\end{tabular}

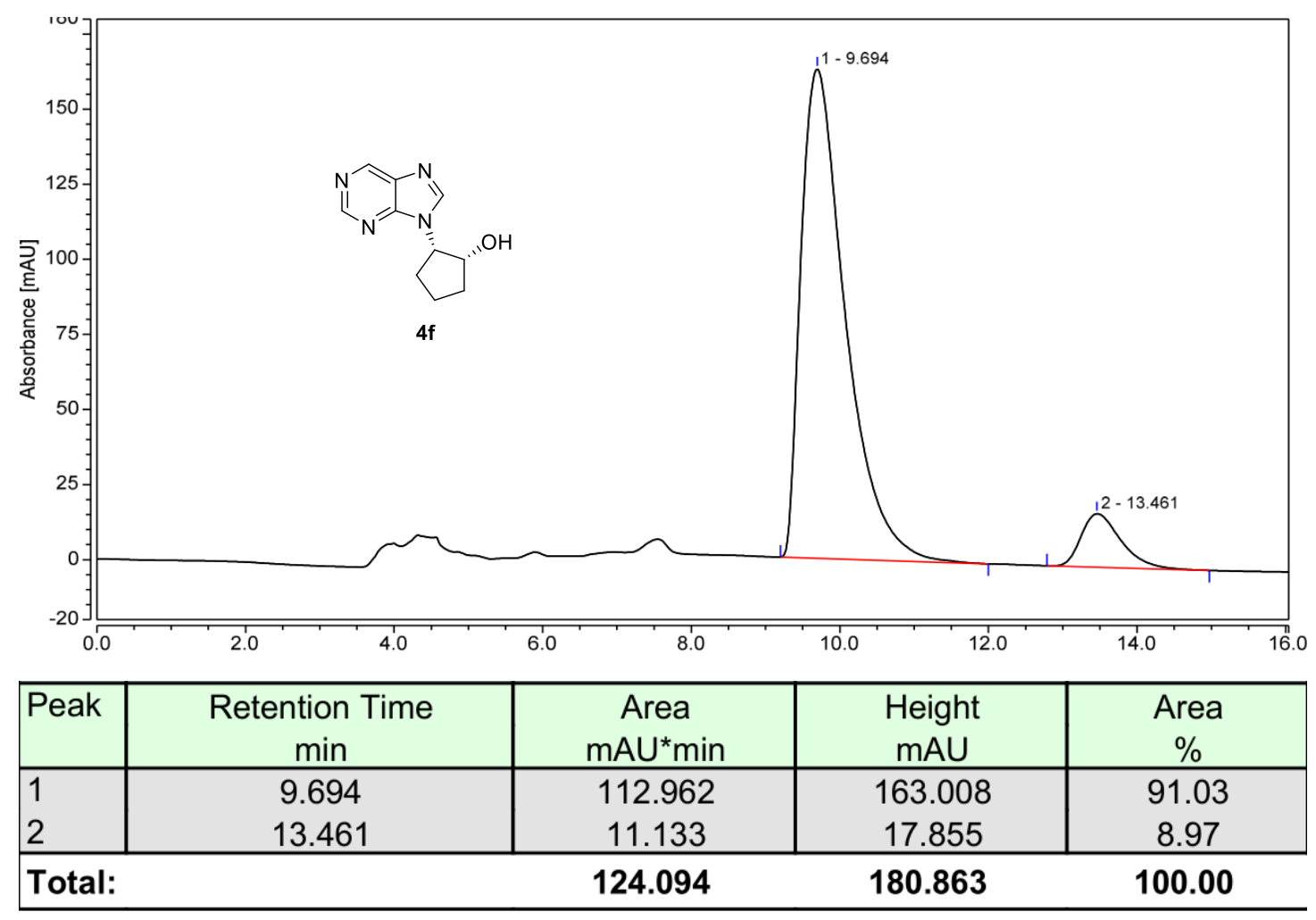




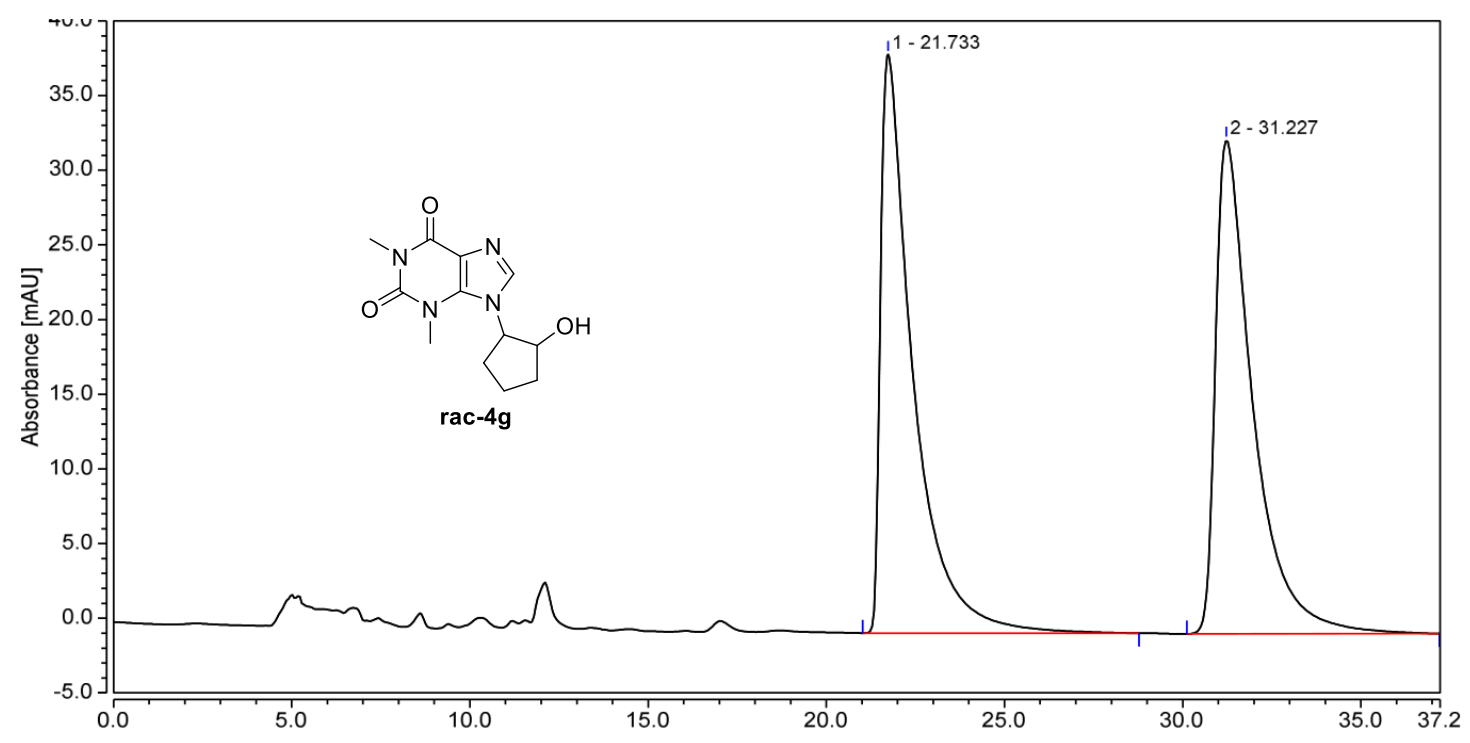

\begin{tabular}{|l|c|c|c|c|}
\hline Peak & $\begin{array}{c}\text { Retention Time } \\
\mathrm{min}\end{array}$ & $\begin{array}{c}\text { Area } \\
\mathrm{mAU}^{*} \min \end{array}$ & $\begin{array}{c}\text { Height } \\
\mathrm{mAU}\end{array}$ & $\begin{array}{c}\text { Area } \\
\%\end{array}$ \\
\hline 1 & 21.733 & 41.149 & 38.748 & 50.59 \\
2 & 31.227 & 40.186 & 33.066 & 49.41 \\
\hline \multicolumn{2}{|l}{ Total: } & $\mathbf{8 1 . 3 3 6}$ & $\mathbf{7 1 . 8 1 4}$ & $\mathbf{1 0 0 . 0 0}$ \\
\hline
\end{tabular}

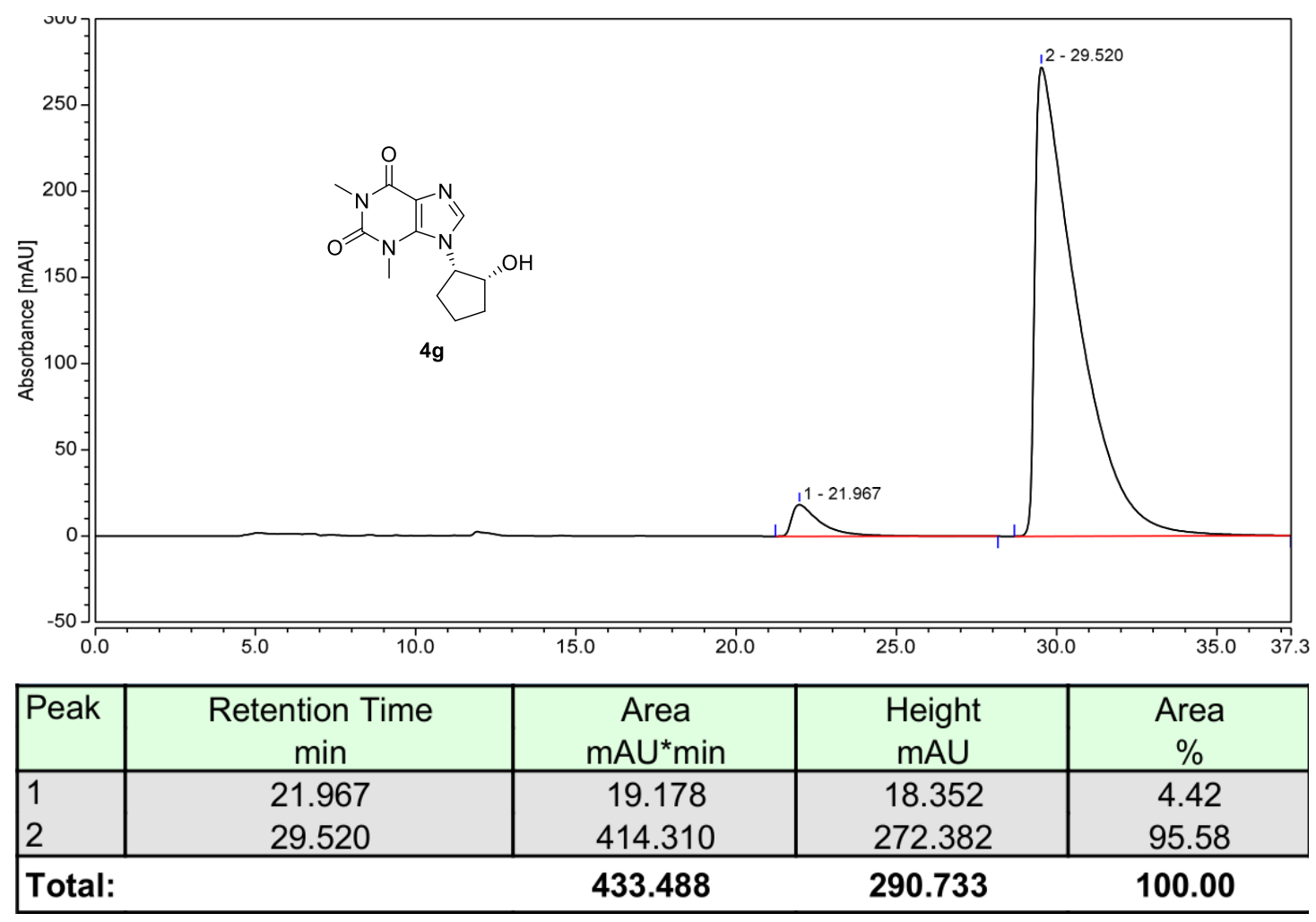




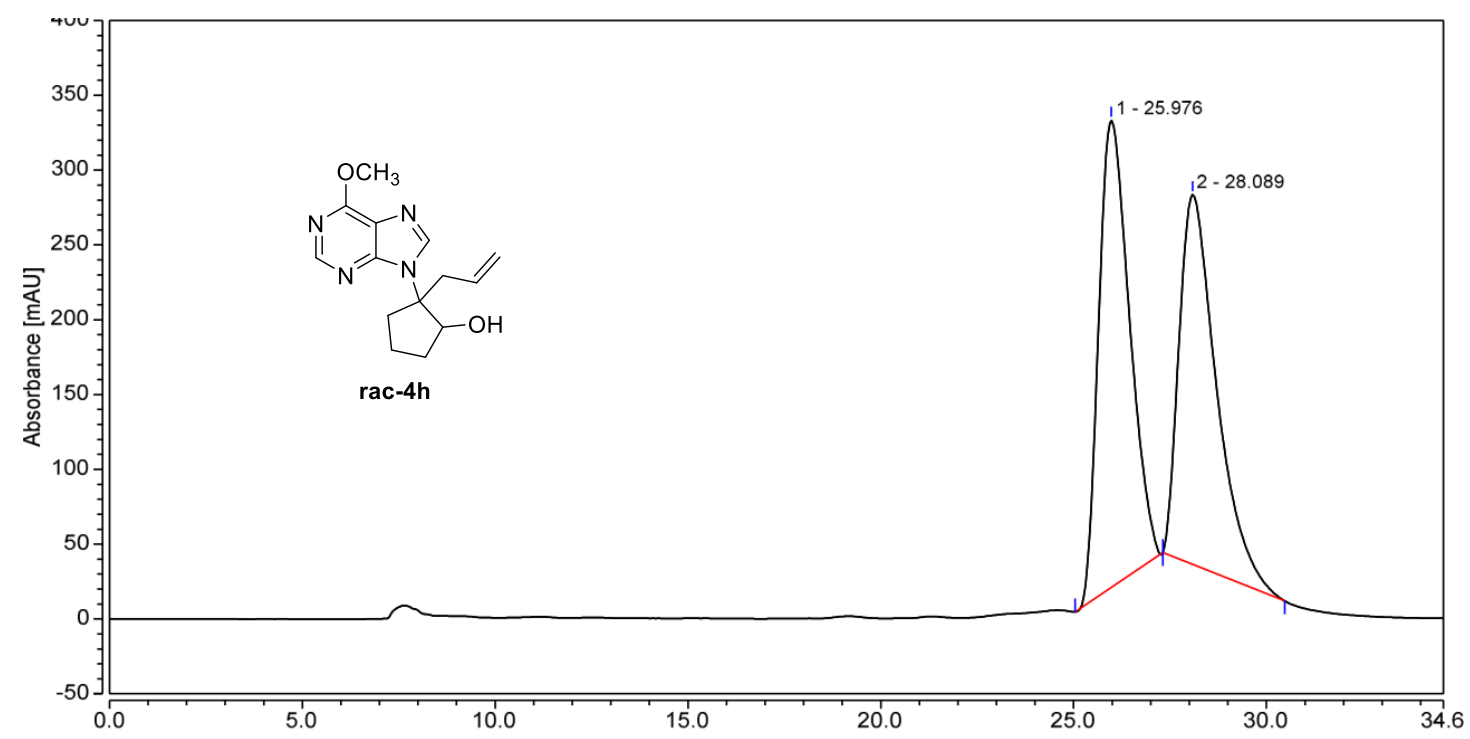

\begin{tabular}{|l|c|c|c|c|c|}
\hline Peak & $\begin{array}{c}\text { Retention Time } \\
\min \end{array}$ & $\begin{array}{c}\text { Area } \\
\text { mAU*min }\end{array}$ & $\begin{array}{c}\text { Height } \\
\text { mAU }\end{array}$ & $\begin{array}{c}\text { Area } \\
\%\end{array}$ & $\begin{array}{c}\text { Height } \\
\%\end{array}$ \\
\hline 1 & 25.976 & 288.682 & 312.333 & 51.02 & 55.82 \\
2 & 28.089 & 277.087 & 247.162 & 48.98 & 44.18 \\
\hline \multicolumn{2}{|l}{ Total: } & $\mathbf{5 6 5 . 7 6 9}$ & $\mathbf{5 5 9 . 4 9 5}$ & $\mathbf{1 0 0 . 0 0}$ & $\mathbf{1 0 0 . 0 0}$ \\
\hline
\end{tabular}

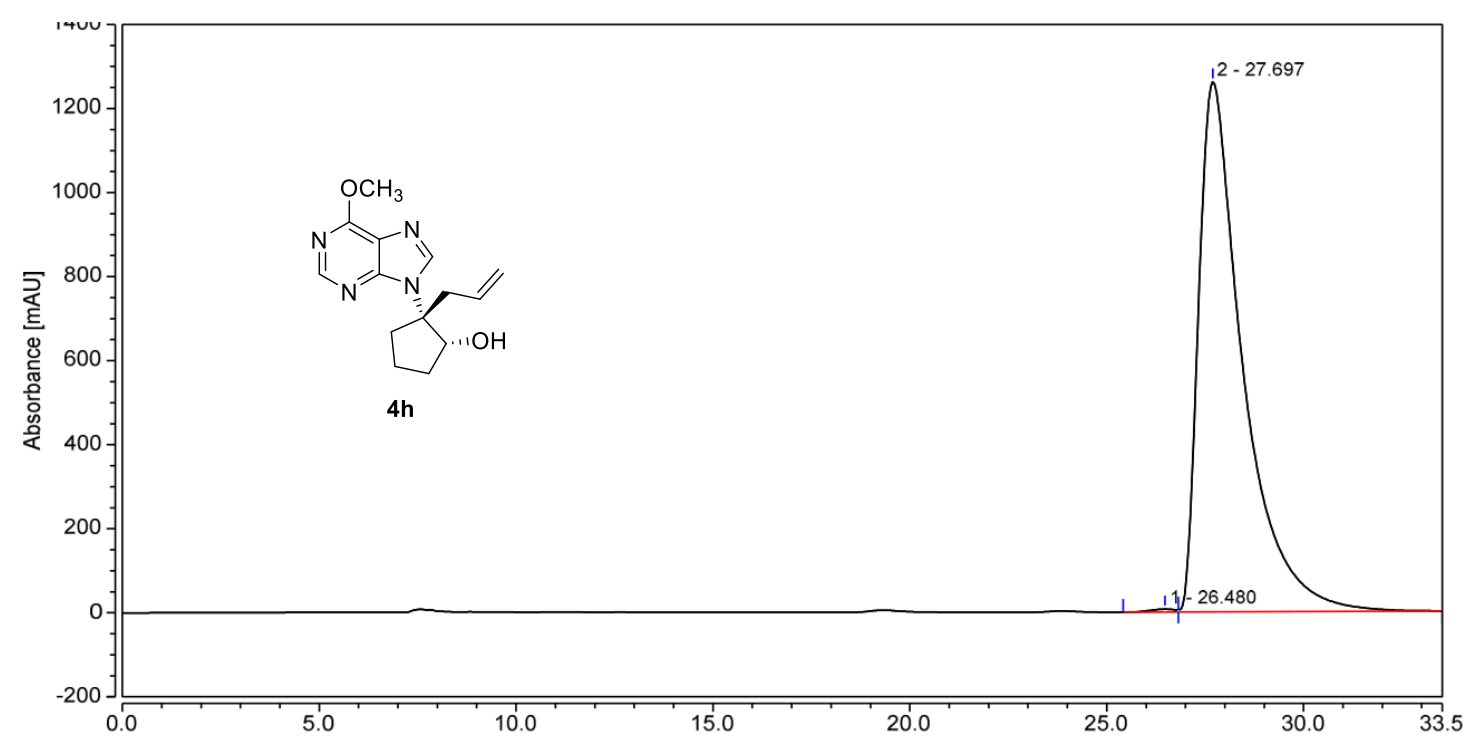

\begin{tabular}{|l|c|c|c|c|c|}
\hline Peak & $\begin{array}{c}\text { Retention Time } \\
\text { min }\end{array}$ & $\begin{array}{c}\text { Area } \\
\mathrm{mAU}^{*} \min \end{array}$ & $\begin{array}{c}\text { Height } \\
\mathrm{mAU}\end{array}$ & $\begin{array}{c}\text { Area } \\
\%\end{array}$ & $\begin{array}{c}\text { Height } \\
\%\end{array}$ \\
\hline 1 & 26.480 & 5.286 & 7.230 & 0.32 & 0.57 \\
2 & 27.697 & 1636.296 & 1261.728 & 99.68 & 99.43 \\
\hline \multicolumn{2}{l|}{ Total: } & $\mathbf{1 6 4 1 . 5 8 2}$ & $\mathbf{1 2 6 8 . 9 5 8}$ & $\mathbf{1 0 0 . 0 0}$ & $\mathbf{1 0 0 . 0 0}$ \\
\hline
\end{tabular}




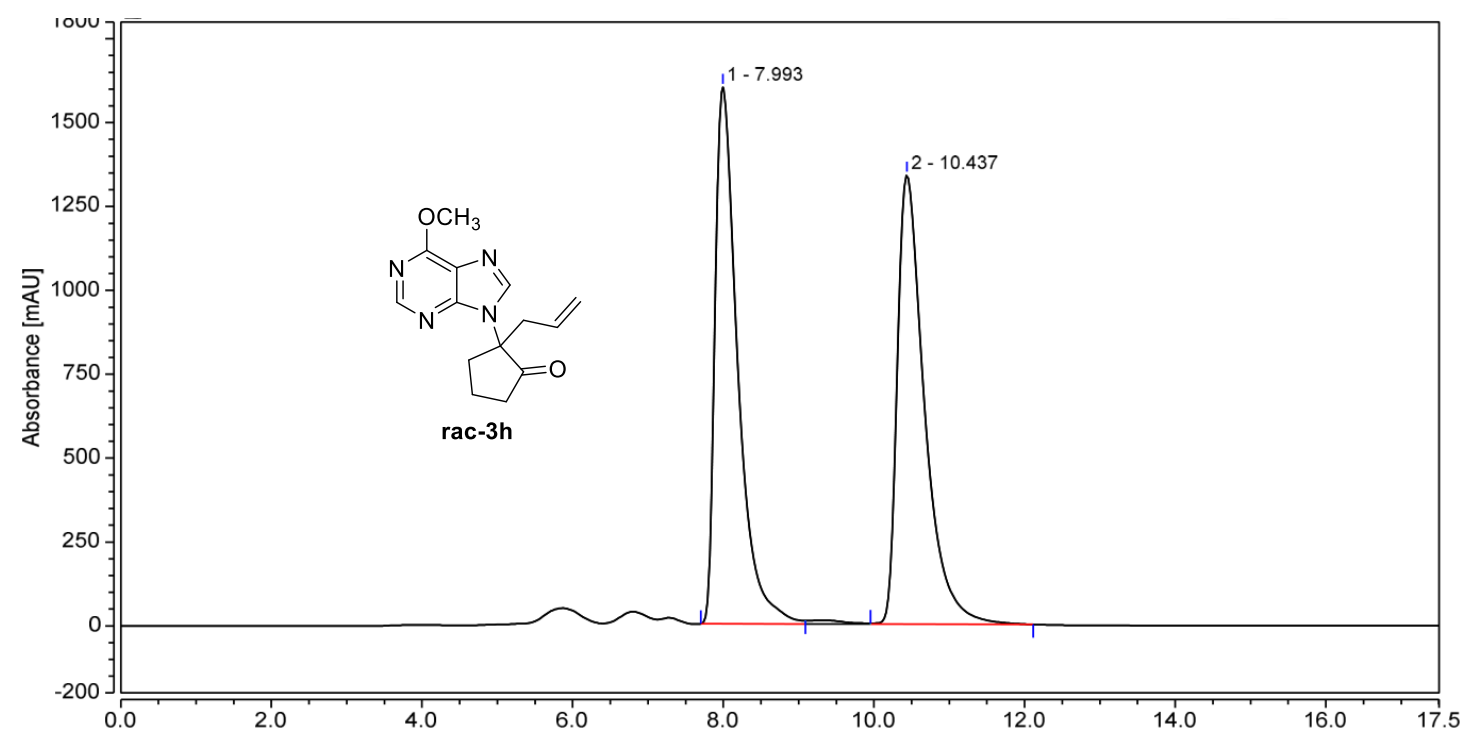

\begin{tabular}{|l|c|c|c|c|c|}
\hline Peak & $\begin{array}{c}\text { Retention Time } \\
\min \end{array}$ & $\begin{array}{c}\text { Area } \\
\text { mAU*min }\end{array}$ & $\begin{array}{c}\text { Height } \\
\mathrm{mAU}\end{array}$ & $\begin{array}{c}\text { Area } \\
\%\end{array}$ & $\begin{array}{c}\text { Height } \\
\%\end{array}$ \\
\hline 1 & 7.993 & 560.393 & 1599.761 & 49.94 & 54.43 \\
2 & 10.437 & 561.656 & 1339.193 & 50.06 & 45.57 \\
\hline \multicolumn{2}{|l}{ Total: } & $\mathbf{1 1 2 2 . 0 4 8}$ & $\mathbf{2 9 3 8 . 9 5 4}$ & $\mathbf{1 0 0 . 0 0}$ & $\mathbf{1 0 0 . 0 0}$ \\
\hline
\end{tabular}

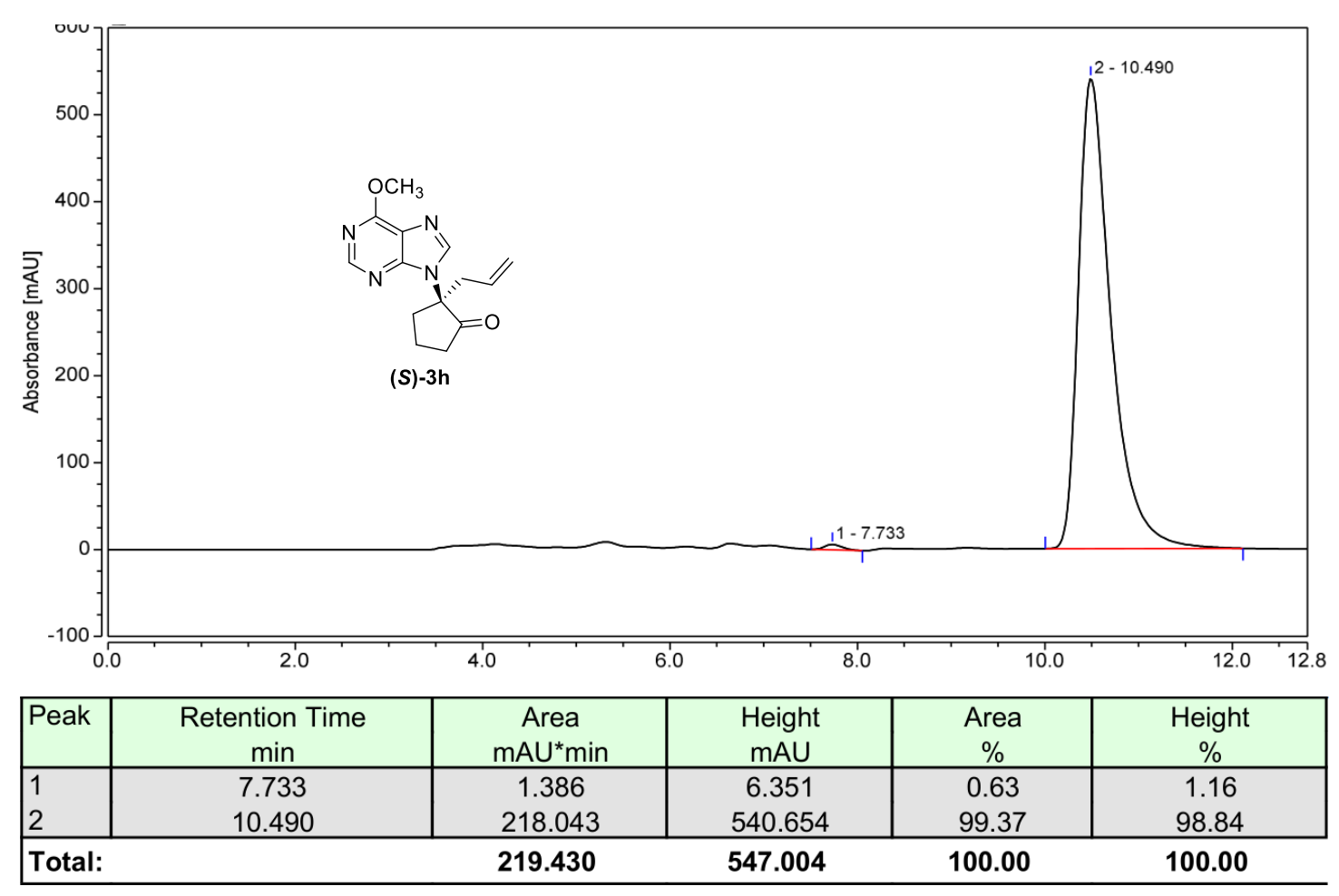



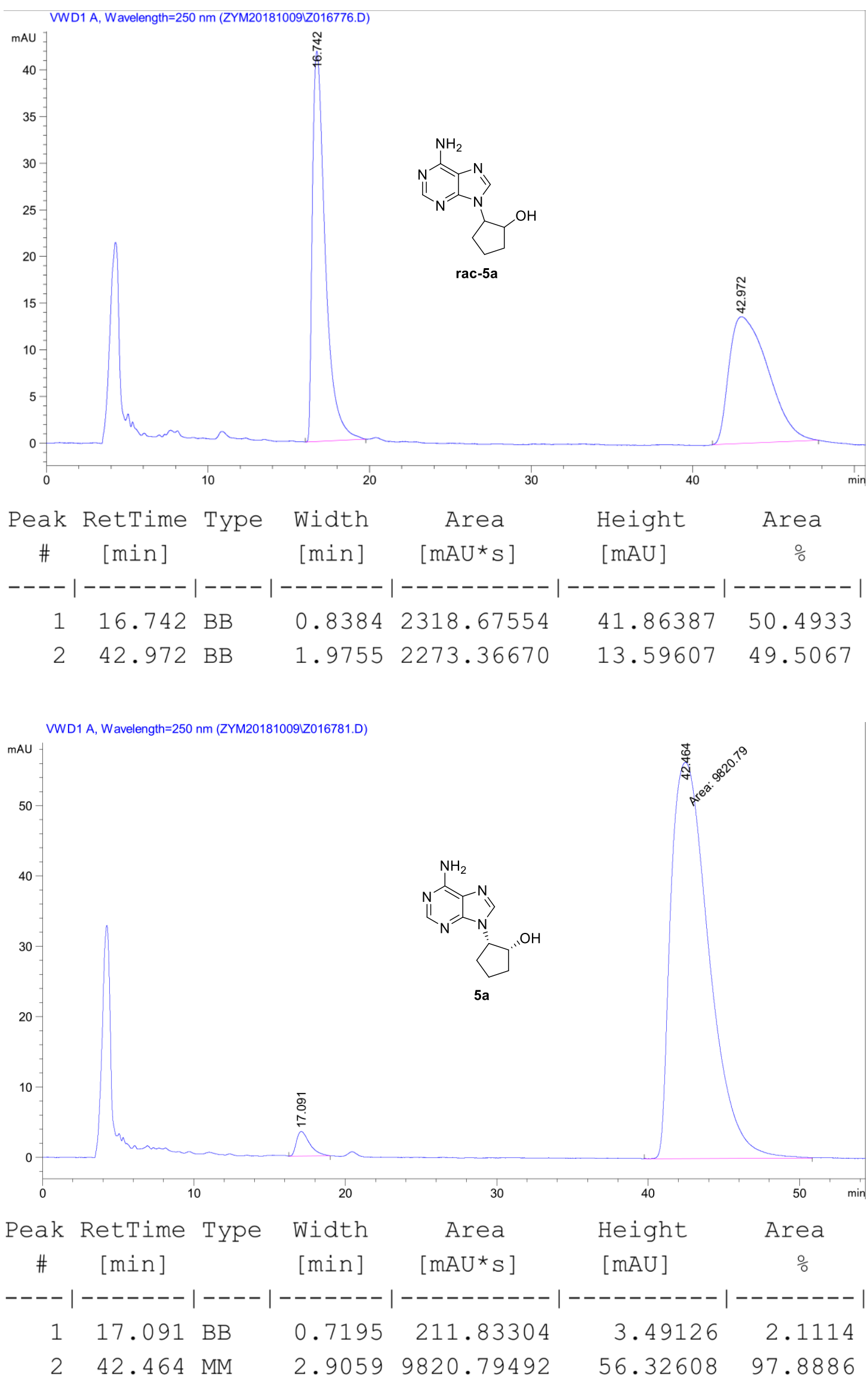


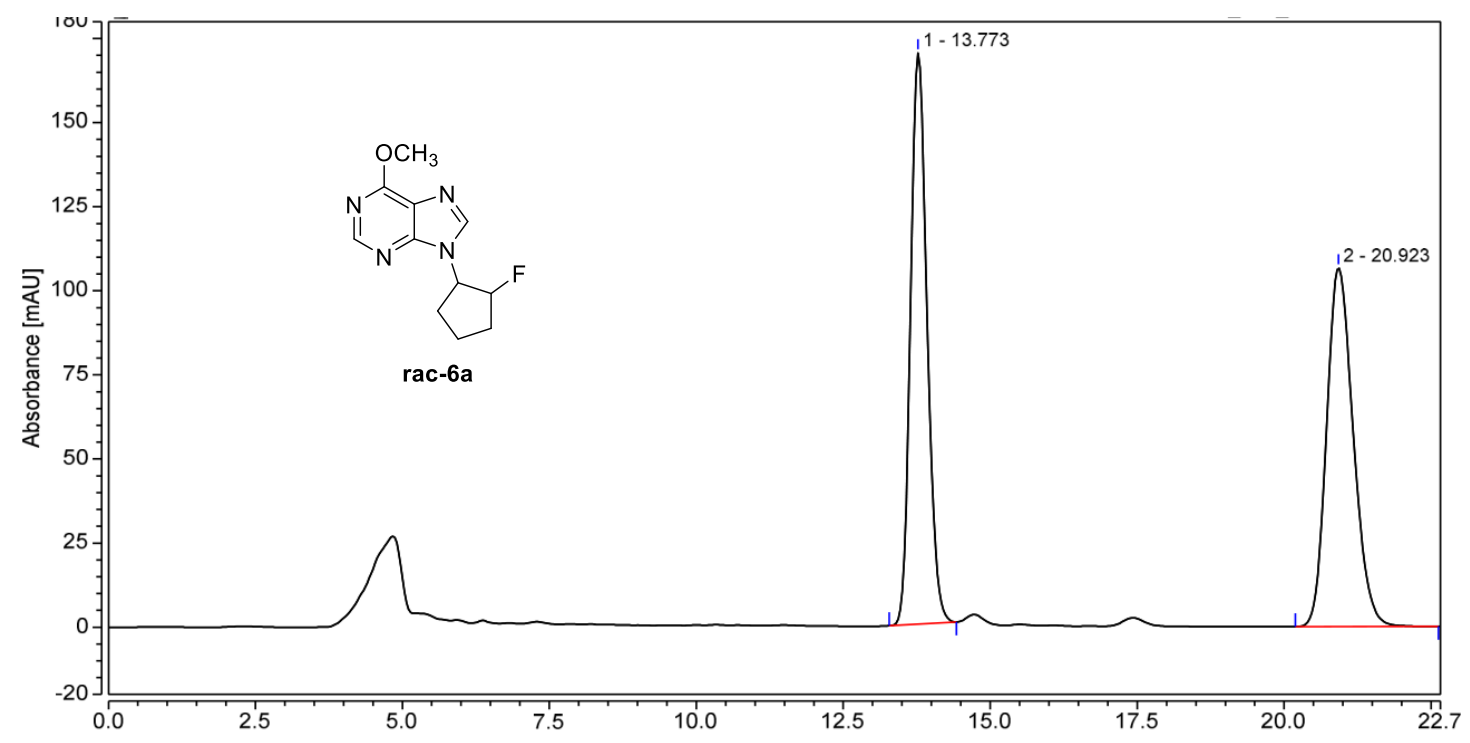

\begin{tabular}{l|c|c|c|c|c|}
\hline Peak & $\begin{array}{c}\text { Retention Time } \\
\text { min }\end{array}$ & $\begin{array}{c}\text { Area } \\
\mathrm{mAU}^{*} \min \end{array}$ & $\begin{array}{c}\text { Height } \\
\mathrm{mAU}\end{array}$ & $\begin{array}{c}\text { Area } \\
\%\end{array}$ & $\begin{array}{c}\text { Height } \\
\%\end{array}$ \\
\hline 1 & 13.773 & 55.188 & 169.933 & 49.60 & 61.43 \\
2 & 20.923 & 56.077 & 106.714 & 50.40 & 38.57 \\
\hline \multicolumn{2}{l}{ Total: } & $\mathbf{1 1 1 . 2 6 5}$ & $\mathbf{2 7 6 . 6 4 7}$ & $\mathbf{1 0 0 . 0 0}$ & $\mathbf{1 0 0 . 0 0}$ \\
\hline
\end{tabular}

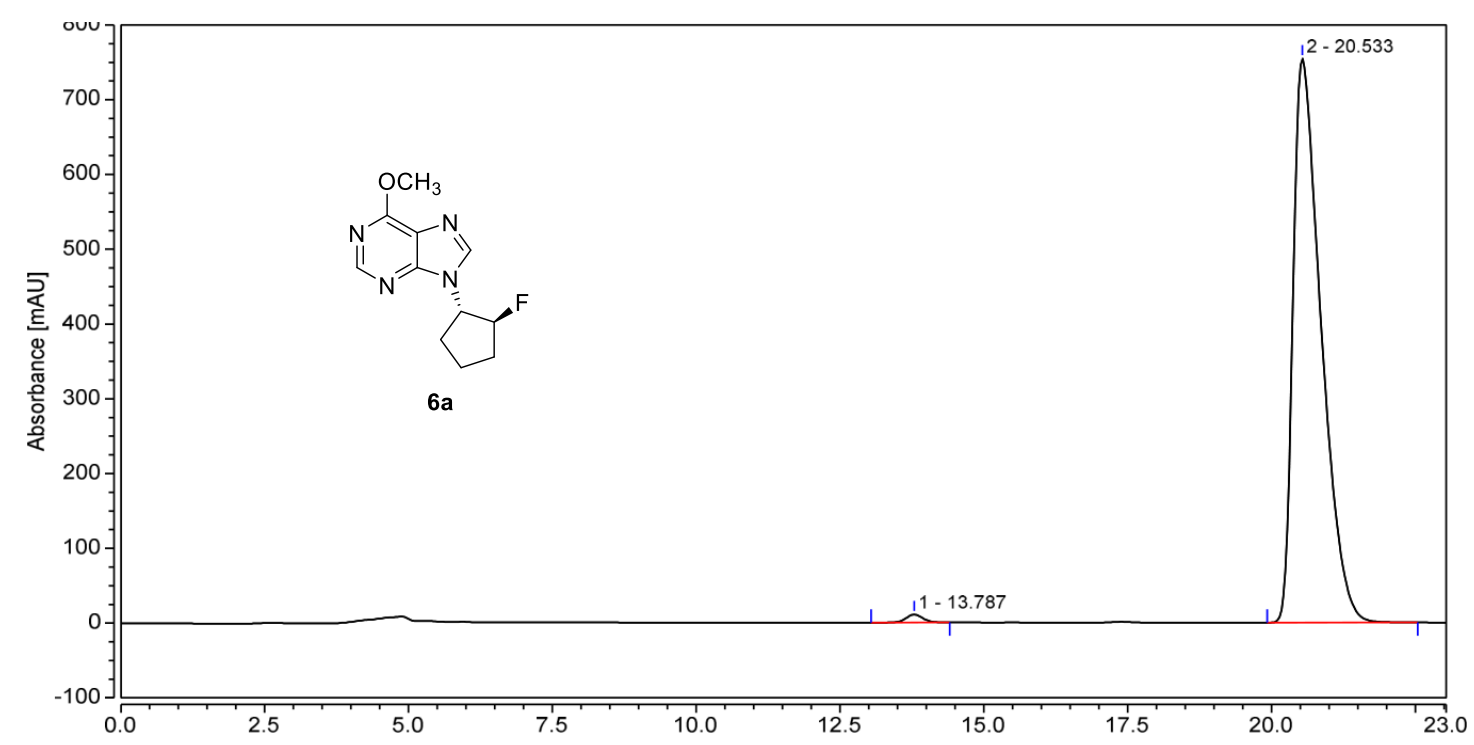

\begin{tabular}{|l|c|c|c|c|c|}
\hline Peak & $\begin{array}{c}\text { Retention Time } \\
\text { min }\end{array}$ & $\begin{array}{c}\text { Area } \\
\mathrm{mAU}^{*} \min \end{array}$ & $\begin{array}{c}\text { Height } \\
\mathrm{mAU}\end{array}$ & $\begin{array}{c}\text { Area } \\
\%\end{array}$ & $\begin{array}{c}\text { Height } \\
\%\end{array}$ \\
\hline 1 & 13.787 & 3.749 & 10.876 & 0.87 & 1.42 \\
2 & 20.533 & 425.884 & 754.428 & 99.13 & 98.58 \\
\hline \multicolumn{2}{l|}{ Total: } & $\mathbf{4 2 9 . 6 3 3}$ & $\mathbf{7 6 5 . 3 0 4}$ & $\mathbf{1 0 0 . 0 0}$ & $\mathbf{1 0 0 . 0 0}$ \\
\hline
\end{tabular}




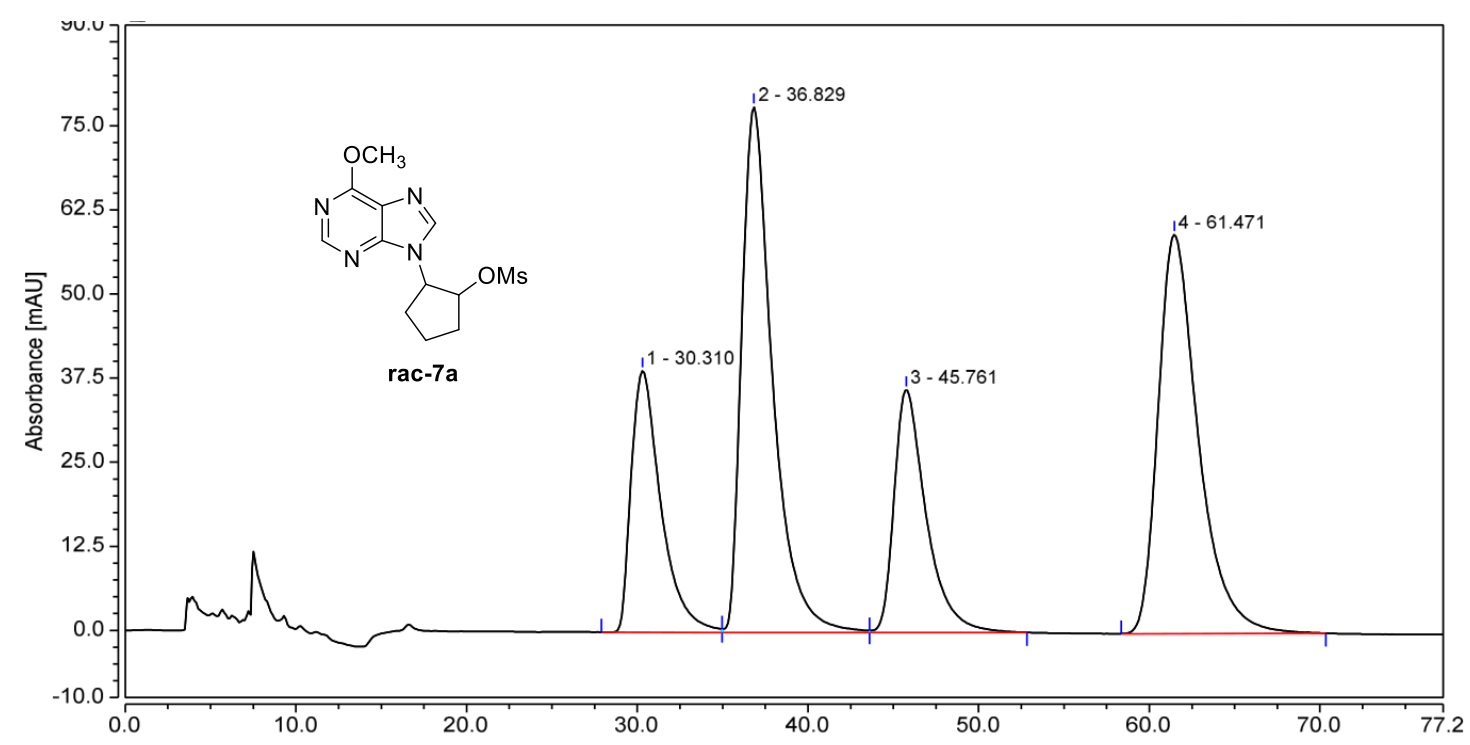

\begin{tabular}{|c|c|c|c|c|c|}
\hline Peak & $\begin{array}{c}\text { Retention Time } \\
\text { min }\end{array}$ & $\begin{array}{c}\text { Area } \\
\mathrm{mAU^{* } \operatorname { m i n }}\end{array}$ & $\begin{array}{l}\text { Height } \\
\text { mAU }\end{array}$ & $\begin{array}{c}\text { Area } \\
\%\end{array}$ & $\begin{array}{c}\text { Height } \\
\%\end{array}$ \\
\hline 1 & 30.310 & 78.496 & 38.880 & 16.26 & 18.30 \\
\hline 2 & 36.829 & 163.897 & 78.127 & 33.95 & 36.77 \\
\hline 3 & 45.761 & 78.493 & 36.114 & 16.26 & 17.00 \\
\hline 4 & 61.471 & 161.944 & 59.366 & 33.54 & 27.94 \\
\hline \multicolumn{2}{|c|}{ Total: } & 482.830 & 212.487 & 100.00 & 100.00 \\
\hline
\end{tabular}

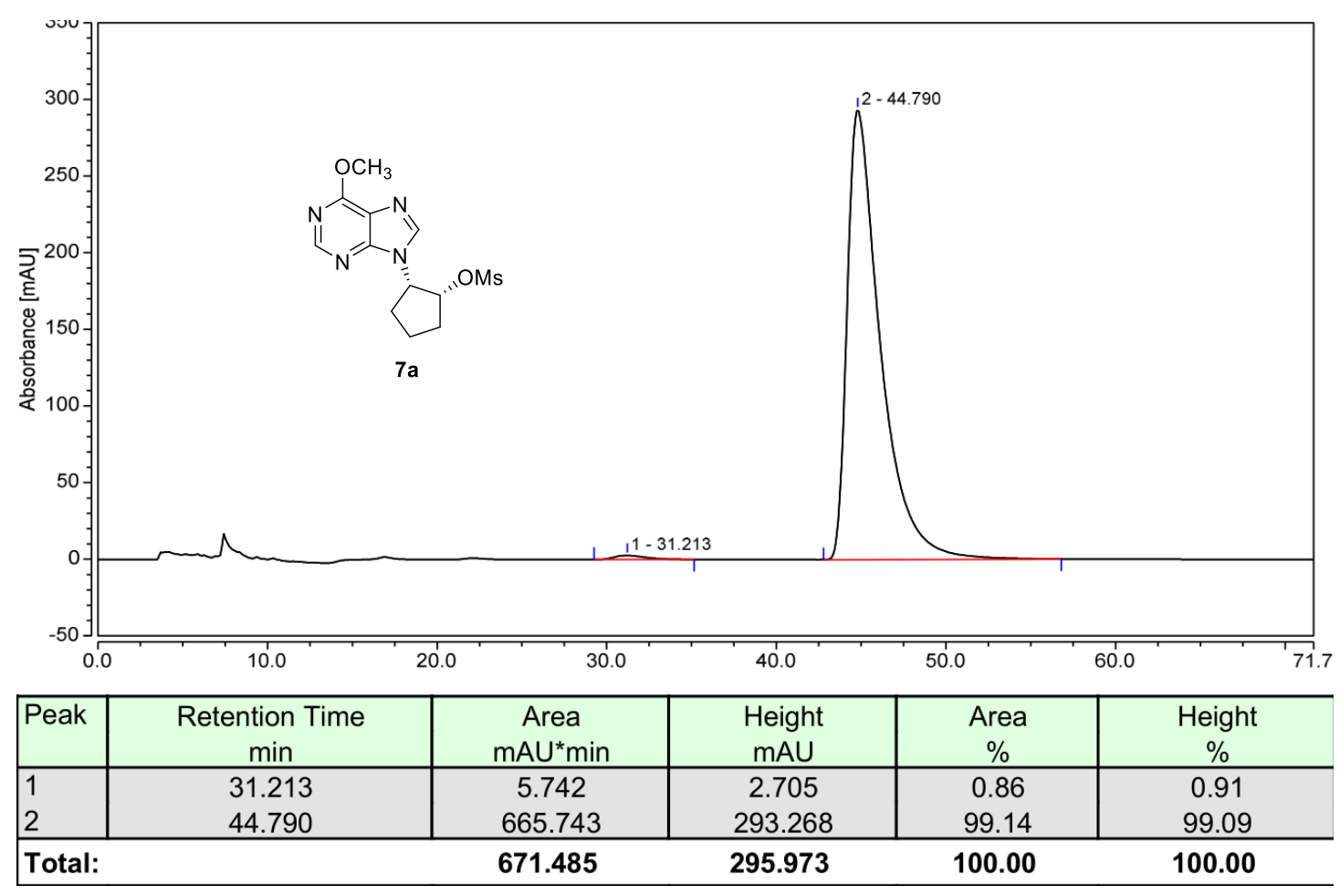




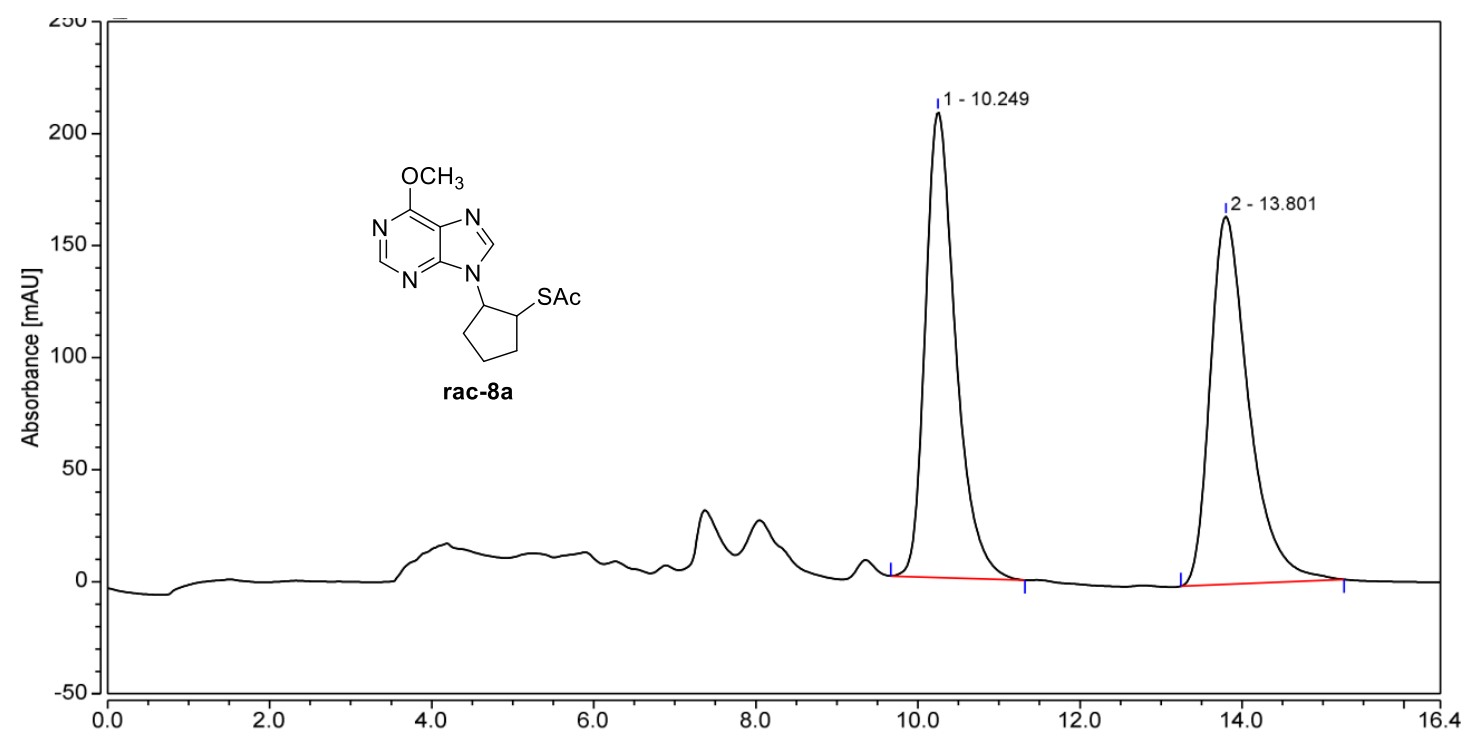

\begin{tabular}{|c|c|c|c|c|c|}
\hline Peak & $\begin{array}{l}\text { Retention Time } \\
\text { min }\end{array}$ & $\begin{array}{c}\text { Area } \\
m A U^{*} \min \end{array}$ & $\begin{array}{l}\text { Height } \\
\text { mAU }\end{array}$ & $\begin{array}{c}\text { Area } \\
\%\end{array}$ & $\begin{array}{c}\text { Height } \\
\%\end{array}$ \\
\hline $\begin{array}{l}1 \\
2\end{array}$ & $\begin{array}{l}10.249 \\
13.801\end{array}$ & $\begin{array}{l}90.301 \\
90.825\end{array}$ & $\begin{array}{l}207.772 \\
164.191\end{array}$ & $\begin{array}{l}49.86 \\
50.14\end{array}$ & $\begin{array}{l}55.86 \\
44.14\end{array}$ \\
\hline \multicolumn{2}{|l|}{ Total: } & 181.127 & 371.963 & 100.00 & 100.00 \\
\hline
\end{tabular}

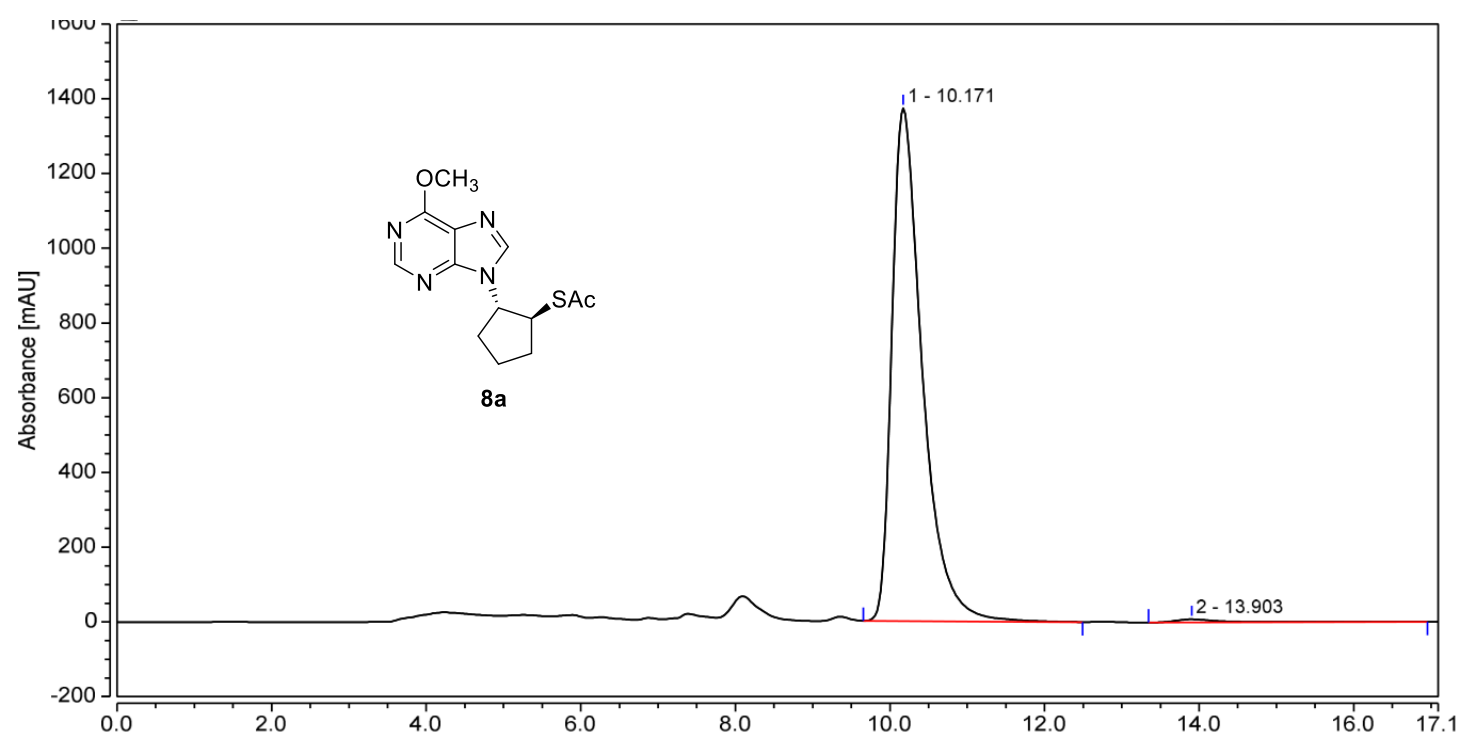

\begin{tabular}{|l|c|c|c|c|c|}
\hline Peak & $\begin{array}{c}\text { Retention Time } \\
\text { min }\end{array}$ & $\begin{array}{c}\text { Area } \\
\mathrm{mAU}^{*} \min \end{array}$ & $\begin{array}{c}\text { Height } \\
\mathrm{mAU}\end{array}$ & $\begin{array}{c}\text { Area } \\
\%\end{array}$ & $\begin{array}{c}\text { Height } \\
\%\end{array}$ \\
\hline 1 & 10.171 & 626.135 & 1372.798 & 98.98 & 99.39 \\
2 & 13.903 & 6.444 & 8.358 & 1.02 & 0.61 \\
\hline \multicolumn{2}{l}{ Total: } & $\mathbf{6 3 2 . 5 7 9}$ & $\mathbf{1 3 8 1 . 1 5 5}$ & $\mathbf{1 0 0 . 0 0}$ & $\mathbf{1 0 0 . 0 0}$ \\
\hline
\end{tabular}



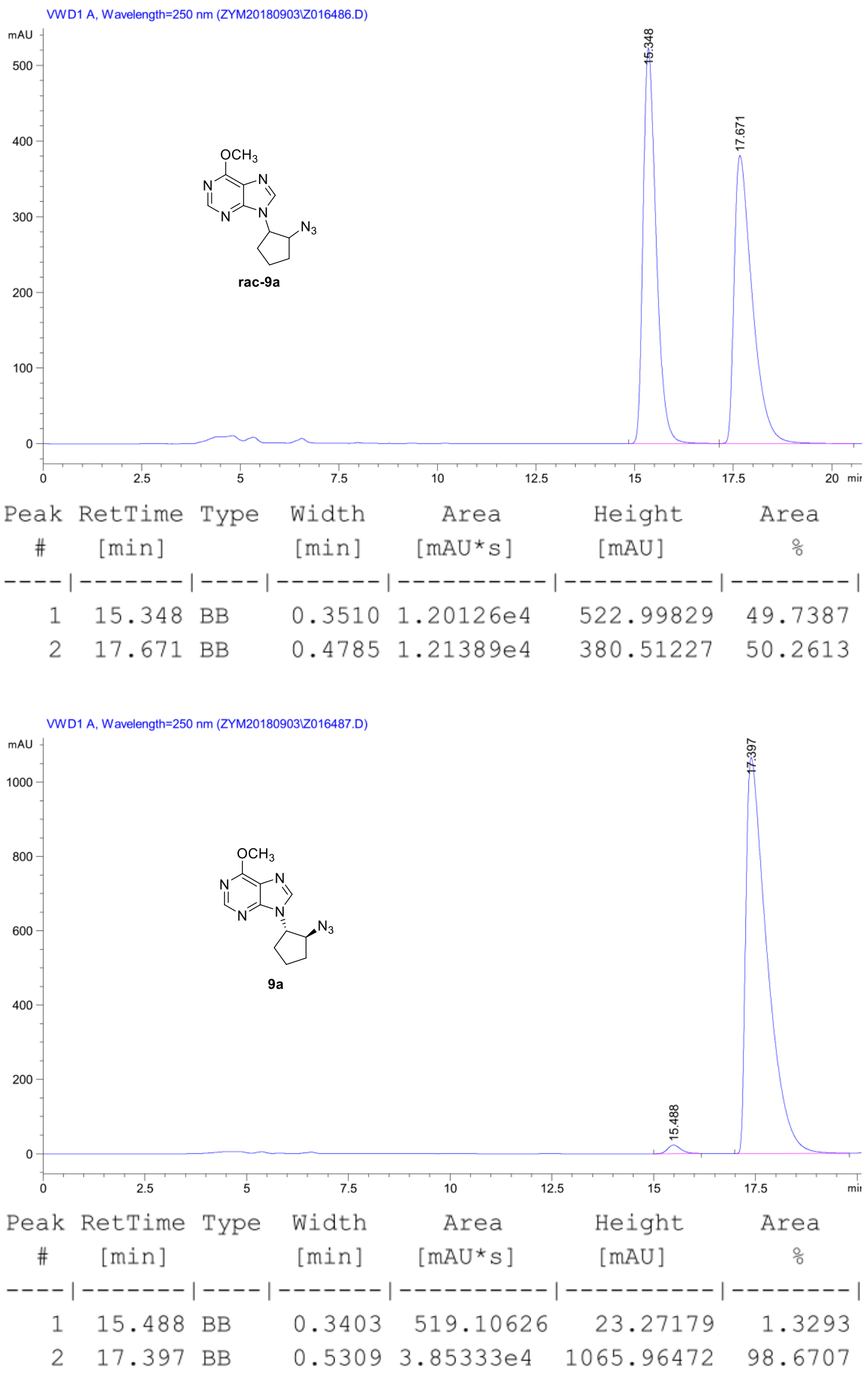

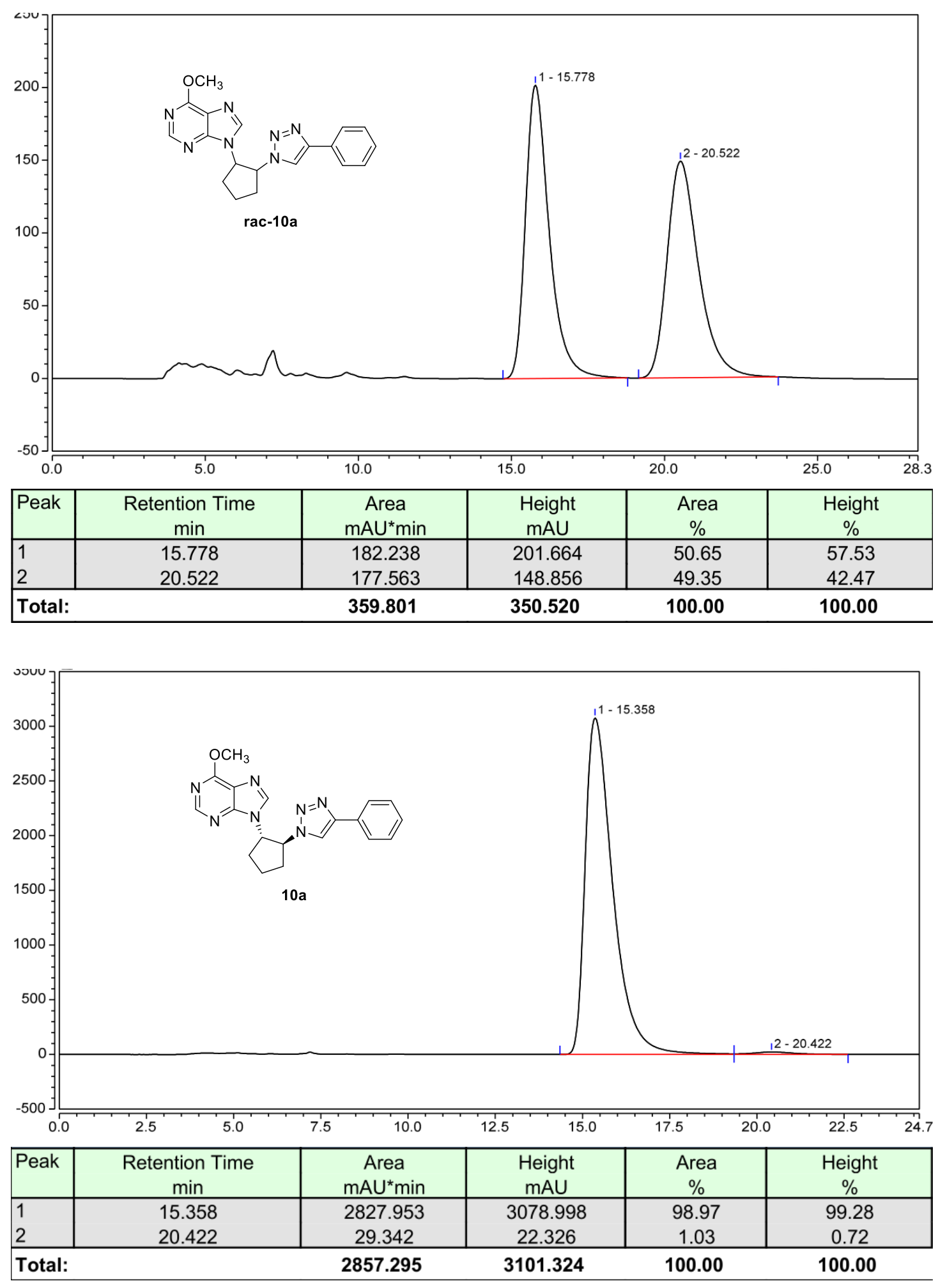

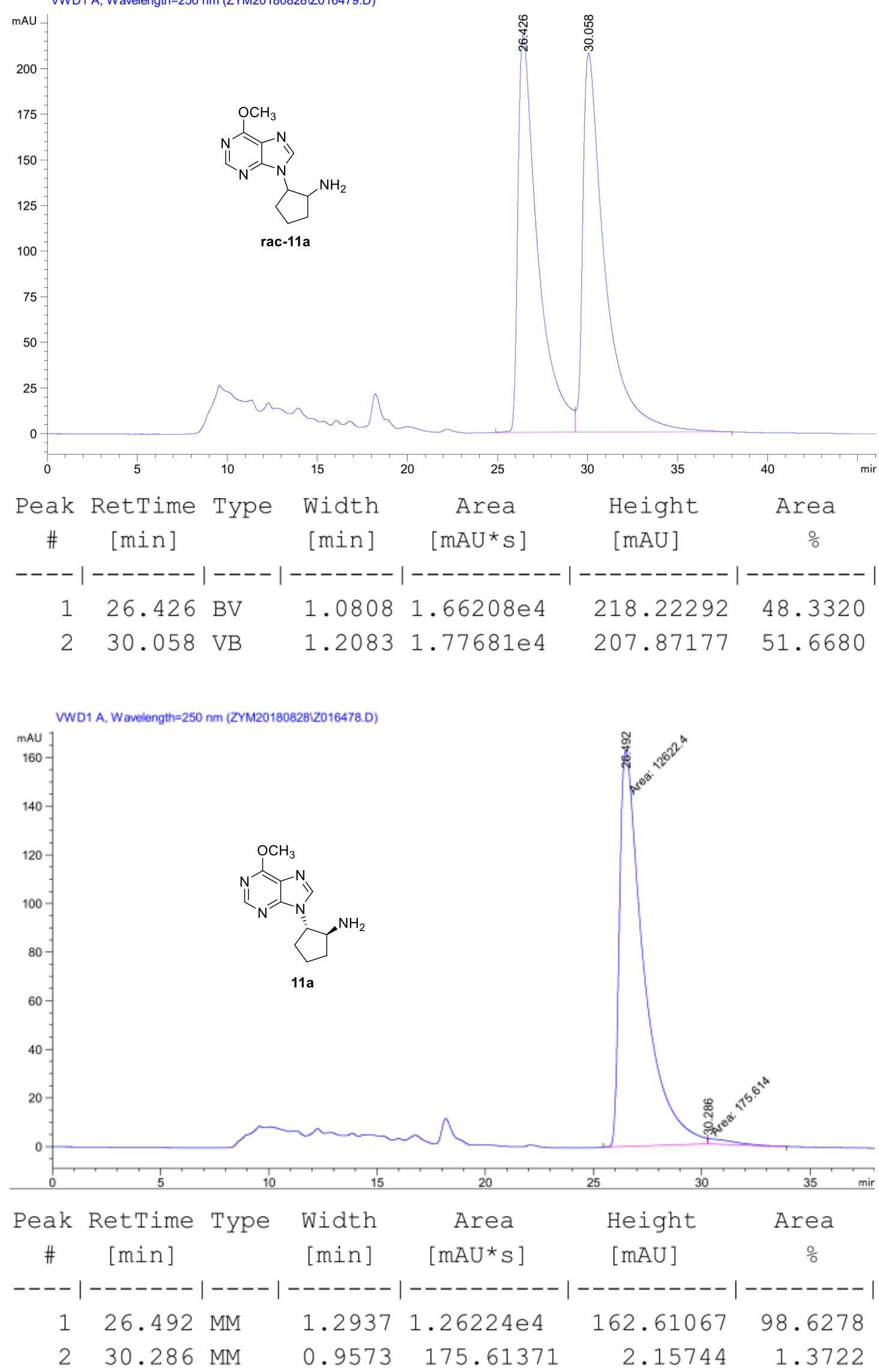


\section{References}

(1) Guha, S. K.; Wu, B.; Kim, B. S.; Baik, W.; Koo, S. Tetrahedron Lett. 2006, 47, 291-293.

(2) Caddick, S.; Parr, N. J.; Pritchard, M. C. Tetrahedron. 2001, 57, 6615-6626.

(3) Guan, Y. Q.; Gao, M.; Deng, X.; Lv, H.; Zhang, X. Chem. Commun. 2017, 53, 8136-8139.

(4) Biggadike, K.; Borthwick, A. D.; Exall, A. M.; Kirk, B. E.; Ward, R. A. J. Chem. Soc., Chem. Commun. 1988, 898-900.

(5) Waske, P. A.; Meyerbroker, N.; Eck, W.; Zharnikov, M. J. Phys. Chem. C. 2012, 116, $13559-13568$.

(6) Berry, M. T.; Castrejon, D.; Hein, J. E. Org. Lett. 2014, 16, 3676-3679. 